\title{
Últimos Levelings: \\ Conceitos, Propriedades, Algoritmos \\ e Aplicações em Processamento \\ e Análise de Imagens
}

Wonder Alexandre Luz Alves

TESE APRESENTADA

AO

Instituto De MatemáticA E EstatísticA

DA

Universidade DE SÃo PAUlo

PARA

OBTENÇÃO DO TÍtUlo

$\mathrm{DE}$

DOUTOR EM CIÊNCIAS

Programa: Ciência da Computação

Orientador: Prof. Dr. Ronaldo Fumio Hashimoto 


\section{Últimos Levelings: \\ Conceitos, Propriedades, Algoritmos \\ e Aplicações em Processamento \\ e Análise de Imagens}

Esta versão da tese contém as correções e alterações sugeridas pela Comissão Julgadora durante a defesa da versão original do trabalho, realizada em 06/08/2015. Uma cópia da versão original está disponível no Instituto de Matemática e Estatística da Universidade de São Paulo.

Comissão Julgadora:

- Prof. Dr. Ronaldo Fumio Hashimoto (orientador) - IME-USP

- Prof. Dr. Paulo André Vechiatto de Miranda - IME-USP

- Prof. Dr. Roberto de Alencar Lotufo - UNICAMP

- Prof. Dr. João Paulo Papa - UNESP

- Prof. Dr. Sidnei Alves de Araújo - PPGIGC-UNINOVE 


\section{Agradecimentos}

Ao Prof. Ronaldo F. Hashimoto, pela paciência e incentivo com que me orientou e pelo esforço para produzirmos este trabalho.

Aos meus colaboradores (em especial, Ronaldo, Beatriz, Alexandre e Edu) que ajudaram a enriquecer esta tese.

Aos meus amigos Maciel, Rafael, Fabiano, Pablo e Deusany pelas nossas reuniões de autômatos.

Aos meus amigos Sidnei, Maysa e Marta pelas ajudas nas revisões de textos e ideias que foram importantes para refinamento deste trabalho.

A Martinha pelo carinho, inventivo, companheirismo e ajuda desde o início do meu doutorado.

Aos meus familiares (em especial, meus irmãos Willy e Larissa, e meus pais França e Lete) por entenderem minhas ausências e estarem sempre ao meu lado.

Aos amigos do IME-USP, da UNINOVE, a dona Lourdes e os meus cachorros Snow e Mel que estiveram sempre presentes e diretamente me ajudaram em diversos momentos difíceis.

E finalmente, aos membros da banca examinadora, pelas sugestões e contribuições que enriqueceram bastante esta tese.

A todos, muito obrigado! 
ALVES, W. A. L., Últimos Levelings: Conceitos, Propriedades, Algoritmos e Aplicações em Processamento e Análise de Imagens. 2015, 138 f. Tese (Doutorado) - Instituto de Matemática e Estatística, Universidade de São Paulo, São Paulo, 2015.

Em Morfologia Matemática diversos operadores são definidos pela diferença entre outros dois operadores, como por exemplo, o gradiente morfológico, definido como a diferença entre a dilatação e a erosão. Estes operadores são denominados operadores residuais, sendo alguns deles definidos por valores residuais extraídos de famílias indexadas de operadores, como por exemplo, o esqueleto por discos maximais e a última abertura. Neste sentido, visa-se neste trabalho investigar a extração de informações residuais em famílias indexadas de operadores. Mais precisamente, em famílias de operadores conexos conhecidos como levelings. Os levelings são operadores que não criam novas estruturas (contornos e extremos regionais) e seus valores são limitados pelos valores da imagem de referência. Assim, é apresentada nesta tese uma classe de operadores residuais denominada últimos levelings, a qual consiste de poderosos operadores residuais definidos a partir de resíduos gerados por operadores consecutivos de um espaço de escala baseado em levelings. Dessa forma, objetos contrastantes podem ser detectados se relevantes resíduos são gerados quando eles são filtrados por um desses levelings. Os valores residuais revelam importantes informações sobre contrastes presentes em uma imagem. Além dos valores residuais, outras informações associadas com eles podem ser obtidas no momento da extração residual, tais como os índices dos operadores que produziram os valores residuais. Com base nessas considerações, as principais contribuições originais desta pesquisa, incluem: ( $i$ ) demonstrar que árvores construídas a partir de conjuntos de níveis representam espaços de escalas baseados em levelings; (ii) introduzir a classe dos últimos levelings, passando por definições, conceitos, algoritmos, propriedades e relações com outros operadores conhecidos na literatura; (iii) apresentar estratégias para construção de operadores últimos levelings. Por fim, são apresentadas aplicações dos últimos levelings em problemas de análise e processamento de imagens.

Palavras-chave: operadores residuais, operadores levelings, árvores morfológicas, últimos levelings. 
ALVES, W. A. L., Ultimate Levelings: Concepts, Properties, Algorithms and Applications for Image Processing and Analysis. 2015, 138 f. Tese (Doutorado) - Instituto de Matemática e Estatística, Universidade de São Paulo, São Paulo, 2015.

In Mathematical Morphology several operators are defined by the difference between two operators, such as morphological gradient, defined as the difference between the dilation and erosion. These operators are called residual operators, being that some are defined by the extracted residual values from of an indexed family of operators, for example, the skeleton by maximal discs and the ultimate opening. In this sense, we intend to investigate the extraction of residual information in families of operators. More precisely, in families of connected operators known as levelings. The levelings are operators that do not create new structures (contours and regional extremes) and their values are limited by the values of the reference image. Thus, we present in this thesis a class of residual operators named ultimate levelings, which consist of powerful residual operators defined from a scale space based on levelings. With a multi-scale approach, these operators analyze an image under a series of levelings. Thus, contrasted objects can be detected if a relevant residue is generated when they are filtered out by one of these levelings. The residual values reveal important informations about contrasts present in an image. In addition of residual values, other information associated with them can be obtained at the time of extraction residual, such as the indexes of operators who produced the residual values. Based on these considerations, the main original contributions of this research include: $(i)$ demonstrate that the trees constructed from level sets represent an scale space based on levelings; (ii) introduce the class of levelings ultimate, passing by definitions, concepts, algorithms, properties and relationships with other known operators in the literature; (iii) show some strategies for building levelings ultimate operators. Finally, we present applications of levelings ultimate in problem of image processing and analysis.

Keywords: residual operators, leveling operators, morphological trees, ultimate levelings. 
Lista de Abreviaturas $\quad$ xi

Lista de Símbolos $\quad$ xiii

Lista de Algoritmos $\quad$ xvii

Lista de Figuras $\quad$ xix

Lista de Tabelas $\quad$ xxiii

1 Introdução $\quad 1$

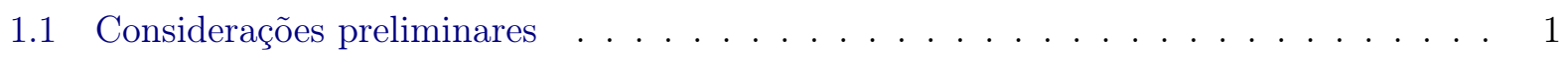

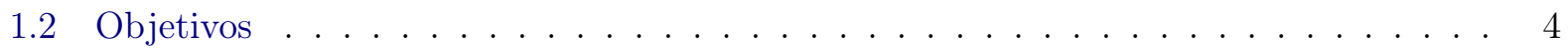

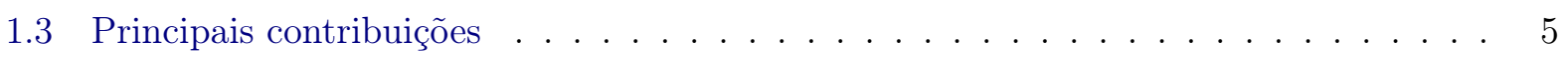

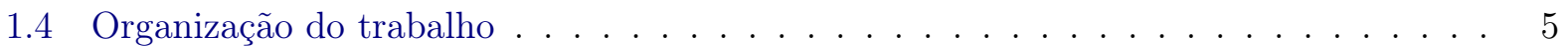

$\begin{array}{lll}\text { I } & \text { Conceitos sobre imagens, operadores e representações } & 7\end{array}$

2 Conceitos sobre imagens e operadores morfológicos $\quad 9$

2.1 Definições básicas sobre imagens . . . . . . . . . . . . . . . . . . . . 9

2.2 Operadores morfológicos . . . . . . . . . . . . . . . . . . 11

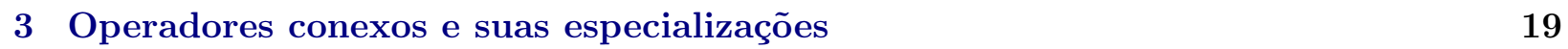

3.1 Conceitos básicos sobre operadores conexos e suas especializações . . . . . . . . . . . 19

3.2 Operadores vistos como relações binárias . . . . . . . . . . . . . . . . . . 22

3.3 Propriedades com relação as extremas regionais . . . . . . . . . . . . . . . . . 24

3.4 Algumas propriedades algébricas sobre os operadores conexos, levelings e monotone

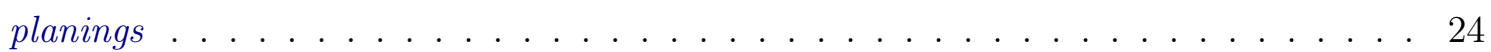

3.5 Relações entre levelings e reconstruções por dilatações e erosões . . . . . . . . . . . . . . . . . . . . .

3.6 Espaço de escala com base em levelings . . . . . . . . . . . . . . . . . . . 27

4 Representações de imagens por meio de árvores baseadas em conjuntos de níveis 29

4.1 Conceitos de árvores construídas por conjuntos parcialmente ordenados . . . . . . . . 29

4.2 Árvores baseadas em conjuntos de níveis . . . . . . . . . . . . . . . . . . . . 32

4.2 .1 Árvore de componentes . . . . . . . . . . . . . . . . . . . . . 34

4.2 .2 Árvore de formas . . . . . . . . . . . . . . . . . . . . . . . . . 40

4.2 .3 Reconstrução de árvores podadas . . . . . . . . . . . . . . . . . . . . 48 
II Últimos levelings: conceitos, propriedades e algoritmos

5 Representações de espaço de escala baseado em levelings através de hierarquias dos conjuntos de níveis

5.1 Relações entre reconstruções de árvores podadas e operadores levelings . . . . . . . . 53

5.2 Operadores morfológicos obtidos por meio de reconstruções de árvores podadas . . 59

5.2 .1 Operadores baseados em imagens marcadoras . . . . . . . . . . . . . . . 59

5.2 .2 Operadores baseados em atributos . . . . . . . . . . . . . 63

5.3 Espaço de escala baseado em levelings por meio de podas sucessivas . . . . . . . . . . 64

5.3 .1 Espaço de escala baseado em atributos . . . . . . . . . . . . . . . . . 65

5.3.2 Espaço de escala baseado em imagens marcadoras . . . . . . . . . . . . . . . 66

6 Últimos levelings $\quad 69$

6.1 Das últimas aberturas e últimos fechamentos para os últimos levelings . . . . . . . . 69

6.2 Propriedade associadas aos operadores últimos levelings . . . . . . . . . . . . . 72

6.2.1 Propriedades sobre preservação de estruturas . . . . . . . . . . . . . . . . 72

6.2.2 Propriedade a respeito de complementação . . . . . . . . . . . . . . . . . 75

6.3 Algoritmo para construção dos últimos levelings . . . . . . . . . . . . . . . . 76

6.4 Informações associadas aos últimos levelings . . . . . . . . . . . . . . . . 80

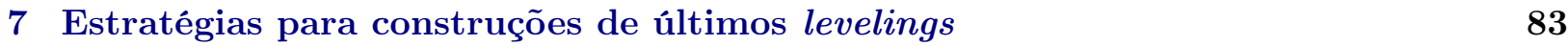

7.1 Estratégias para escolher a família de primitivas . . . . . . . . . . . . . . . 83

7.1.1 Primitivas baseadas em atributos . . . . . . . . . . . . . . . 85

7.1.2 Primitivas baseadas em imagens marcadoras . . . . . . . . . . . . . . . . 88

7.2 Estratégias para filtrar resíduos indesejáveis . . . . . . . . . . . . . . . . 90

7.2.1 Filtragem com base em vetor de atributos . . . . . . . . . . . . . . . 91

$7.2 .2 \quad$ Filtragem com base em regiões estáveis . . . . . . . . . . . . . . . . . . 94

$\begin{array}{ll}\text { III Aplicações } & 99\end{array}$

8 Segmentação de vasos sanguíneos em imagens de retinas $\quad 101$

8.1 Introdução . . . . . . . . . . . . . . . . . . . . . . . . . . . . 101

8.2 Abordagem proposta . . . . . . . . . . . . . . . . . . 102

8.3 Experimentos e resultados . . . . . . . . . . . . . . . . . . . 104

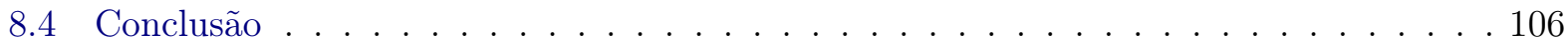

9 Binarização de texto em imagens de documentos históricos $\quad 107$

9.1 Introdução . . . . . . . . . . . . . . . . . . . . . . . . . . . 107

9.2 Abordagem proposta . . . . . . . . . . . . . . . . 108

9.3 Experimentos e resultados . . . . . . . . . . . . . . . . . . . . . . 109

9.4 Conclusão . . . . . . . . . . . . . . . . . . . . . . . . . 111

10 Localização de textos em imagens de cenas 113

10.1 Introdução . . . . . . . . . . . . . . . . . . . . . . . . . 113

10.2 Abordagem proposta . . . . . . . . . . . . . . . . . 114 
10.3 Experimentos e resultados . . . . . . . . . . . . . . . . . . . 117

10.4 Conclusão . . . . . . . . . . . . . . . . . . . . . . . . . . 119

11 Conclusões $\quad 121$

11.1 Trabalhos futuros . . . . . . . . . . . . . . . . . . . . 121

IV Apêndices $\quad 123$

A Produções durante o doutorado 125

$\begin{array}{ll}\text { Referências Bibliográficas } & 127\end{array}$

$\begin{array}{ll}\text { İndice Remissivo } & 137\end{array}$ 


\section{Lista de Abreviaturas}

CC Componente Conexo

DIBCO Document Image Binarization Contest

(em português - concurso de binarização de imagens de documentos)

DRIVE Digital Retinal Images for Vessel Extraction

(em português - Imagens digital retina para extração vasos sanguíneos)

EE Elemento Estruturante

LIFO Acrônimo para a expressão inglesa Last In, First Out (em português - último a entrar, primeiro a sair)

MM Morfologia Matemática

MSER Maximally Stable Extremal Regions

(em português - regiões extremais maximamente estáveis)

OCR Optical Character Recognition

(em português - reconhecimento ótico de caracteres)

pixel Acrônimo para a expressão inglesa Picture Element

(em português - elemento da imagem)

poset Acrônimo para a expressão inglesa partially ordered set

(em português - conjunto parcialmente ordenado)

RGB Red, Green and Blue

(em português - vermelho, verde e azul)

ROC Receiver Operating Characteristic

(em português - característica de operação do receptor)

STARE Structured Analysis of the Retina

(em português - Análise da estrutura da retina)

UAC Ultimate Attribute Closing

(em português - último fechamento por atributo)

UAO Ultimate Attribute Opening

(em português - última abertura por atributo)

UGF Ultimate Grain Filter

(em português - último filtro por grão) 


\section{Lista de Símbolos}

\section{Conceitos básicos}

\begin{tabular}{|c|c|}
\hline $\mathbb{N}$ & Conjunto dos números naturais \\
\hline $\mathbb{Z}$ & Conjunto dos números inteiros \\
\hline $\mathbb{R}$ & Conjunto dos números reais \\
\hline$\emptyset$ & Conjunto vazio \\
\hline$\epsilon$ & É um elemento de \\
\hline$\notin$ & Não é um elemento de \\
\hline$\cap$ & Interseção entre conjuntos \\
\hline$\cup$ & União entre conjuntos \\
\hline$\subseteq$ & É um subconjunto de \\
\hline$\subset$ & É um subconjunto próprio de \\
\hline$\{x: \star\}$ & Conjunto de todos os $x$ que satisfazem uma dada propriedade $\star$ \\
\hline $\min \{x: \star\}$ & É o menor elemento do conjunto $\{x: \star\}$ \\
\hline $\max \{x: \star\}$ & É o maior elemento do conjunto $\{x: \star\}$ \\
\hline$\underset{x \in X}{\operatorname{argmax}}\{f(x)\}$ & Argumento $x \in X$ que maximiza $f(x)$ \\
\hline$\underset{x \in X}{\operatorname{argmin}}\{f(x)\}$ & Argumento $x \in X$ que minimiza $f(x)$ \\
\hline$\backslash$ & Subtração entre os conjuntos, ou seja, $X \backslash Y=\{x: x \in X$ e $x \notin Y\}$ \\
\hline $\mathcal{X}+b$ & O conjunto $\mathcal{X}$ transladado pelo vetor $b$, isto é, $\mathcal{X}+b=\{x+b: x \in \mathcal{X}\}$ \\
\hline$\check{\mathcal{X}}$ & A reflexão do conjunto $\mathcal{X}$, ou seja, $\check{\mathcal{X}}=\{-x: x \in \mathcal{X}\}$ \\
\hline $\mathcal{X}^{c}$ & Complemento do conjunto $\mathcal{X}$, sendo que $\mathcal{X}^{c}=\{x: x \notin \mathcal{X}\}$ \\
\hline$\forall$ & Quantificador lógico universal \\
\hline$\exists$ & Quantificador lógico existencial \\
\hline$\neg$ & Negação de uma proposição lógica \\
\hline$A \Rightarrow B$ & Condicional lógica, se $A$ então $B$ \\
\hline$A \Longleftrightarrow B$ & Bicondicional lógica, $A$ se e somente se $B$ \\
\hline $\mathcal{E}$ & Variável utilizada para representar um relação binária \\
\hline $\mathcal{G}=(\mathcal{V}, \mathcal{E})$ & $\begin{array}{l}\text { Variável utilizada para representar um grafo, de forma que } \mathcal{V} \text { é o conjunto de vértice } \\
\text { e } \mathcal{E} \text { é o conjunto de arestas }\end{array}$ \\
\hline 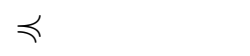 & Símbolo que denota uma relação binária \\
\hline$=1$ & Somatória $1+2+\ldots+k$ \\
\hline & Notação assintótica do Big $\mathrm{O}$ \\
\hline
\end{tabular}




\section{Imagens e operadores}

$\mathcal{D} \quad$ Subconjuntos de $\mathbb{Z}^{2}$ utilizado para representar o domínio de uma imagem

$\mathcal{X}, \mathcal{Y}, \mathcal{Z} \quad$ Variável utilizada para representar subconjuntos de $\mathcal{D}$

$\mathbb{K} \quad$ Subconjuntos de $\mathbb{Z}$ que representa contradomínio da imagem

$\mathcal{P}(\mathcal{D}) \quad$ Conjunto dos subconjuntos de $\mathcal{D}$

$\mathcal{F}(\mathcal{D}) \quad$ Conjunto das imagens definidas com o domínio em $\mathcal{D}$ e o contradomínio em $\mathbb{K}$

$f, g, h \quad$ Variáveis utilizadas para representar imagens.

$p, q \in \mathcal{D} \quad$ Variáveis utilizadas para representar pixels de uma imagem

$\left(p_{x}, p_{y}\right) \in \mathcal{D} \quad$ Notação utilizada para representar as coordenadas horizontal e vertical de um pixel $p$

$f(p) \quad$ Função $f$ aplicada no pixel $p$, ou seja, nível de cinza de $f$ para o pixel $p$

$f \vee g \quad$ Supremo entre duas imagens $f$ e $g$, isto é, $[f \vee g](p)=f(p) \vee g(p)=\max \{f(p), g(p)\}$, para todo $p \in \mathcal{D}$

$f \wedge g \quad$ Ínfimo entre duas imagens $f$ e $g$, isto é, $[f \wedge g](p)=f(p) \wedge g(p)=\min \{f(p), g(p)\}$, para todo $p \in \mathcal{D}$

$f-g \quad$ Imagem obtida pela subtração pixel-a-pixel entre as imagens $f$ e $g$, ou seja, $[f-g](p)=f(p)-g(p)$, para todo $p \in \mathcal{D}$

$f^{c} \quad$ Negativo da imagem $f$, isto é, $f^{c}(p)=K-f(p)$, para todo $p \in \mathcal{D}$

$\mathcal{A} \quad$ Relação de adjacência sobre $\mathcal{D}$

$\mathcal{A}_{4 c} \quad$ Relação de adjacência sobre $\mathcal{D}$ que define a 4-conectividade

$\mathcal{A}_{8 c} \quad$ Relação de adjacência sobre $\mathcal{D}$ que define a 8-conectividade

$(p, q) \in \mathcal{A} \quad$ Par de pixels adjacentes ou vizinhos

$K \quad$ Valor máximo que pode ser atribuído a um pixel

$\mathcal{G}_{f}=(\mathcal{D}, \mathcal{A}) \quad$ Notação utilizada para representar o grafo da imagem $f$, de forma que os pixels são os vértices e as arestas são definidas por uma relação de adjacência $\mathcal{A}$ sobre $\mathcal{D}$

$\mathcal{C C}(\mathcal{X}) \quad$ Conjuntos dos componentes conexos de $\mathcal{X} \subseteq \mathcal{D}$

$\mathcal{Z} \mathcal{P}(f) \quad$ Conjuntos das zonas planas extraídas da imagem $f$

$\mathcal{B} \quad$ Variável utilizada para representar um elemento estruturante

$\psi$ e $\phi \quad$ Variáveis utilizadas para representar operadores de imagens

$\psi(f) \quad$ Operador $\psi$ aplicado na imagem $f$, isto é, a imagem de saída de $\psi$ para a entrada $f$

$[\psi(f)](p) \quad$ Função $\psi(f)$ aplicada no pixel $p$, ou seja, nível de cinza de $\psi(f)$ para o pixel $p$ tal que $\psi(f)$ é a imagem de saída do operador $\psi$ para a entrada $f$

$\mathbb{P}_{f} \quad$ Notação utilizada para representar a partição de $\mathcal{D}$ construída pela zonas planas de $f$

$\mathbb{P}_{f}(p) \quad$ Notação utilizada para representar uma região de $\mathbb{P}_{f}$ contendo o pixel $p$

$\varepsilon_{\mathcal{B}}$ e $\delta_{\mathcal{B}} \quad$ Notações utilizadas para representar os operadores erosão $\varepsilon_{\mathcal{B}}$ e dilatação $\delta_{\mathcal{B}}$ pelo EE $\mathcal{B}$

$\gamma_{\mathcal{B}}$ e $\varphi_{\mathcal{B}} \quad$ Notações utilizadas para representar os operadores abertura $\gamma_{\mathcal{B}}$ e fechamento $\varphi_{\mathcal{B}}$ pelo EE $\mathcal{B}$

$\varepsilon_{\mathcal{B}}^{\infty}$ e $\delta_{\mathcal{B}}^{\infty} \quad$ Notações utilizadas para representar os operadores reconstrução por erosão $\varepsilon_{\mathcal{B}}^{\infty}$ e reconstrução por dilatação $\delta_{\mathcal{B}}^{\infty}$, onde o EE $\mathcal{B}$ define a conectividade da reconstrução

$\gamma_{i}^{\kappa}$ e $\varphi_{i}^{\kappa} \quad$ Notações utilizadas para representar os operadores abertura por atributo $\gamma_{i}^{\kappa}$ e fechamento por atributo $\varphi_{i}^{\kappa}$, onde $i$ é um limiar para o atributo $\kappa$

$\gamma_{\mathcal{B}}^{T H}$ e $\varphi_{\mathcal{B}}^{T H} \quad$ Notações utilizadas para representar os operadores residuais abertura top-hat $\gamma_{\mathcal{B}}^{T H}$ e fechamento top-hat $\varphi_{\mathcal{B}}^{T H}$ por um EE $\mathcal{B}$ 


\section{Representação por meio de árvores}

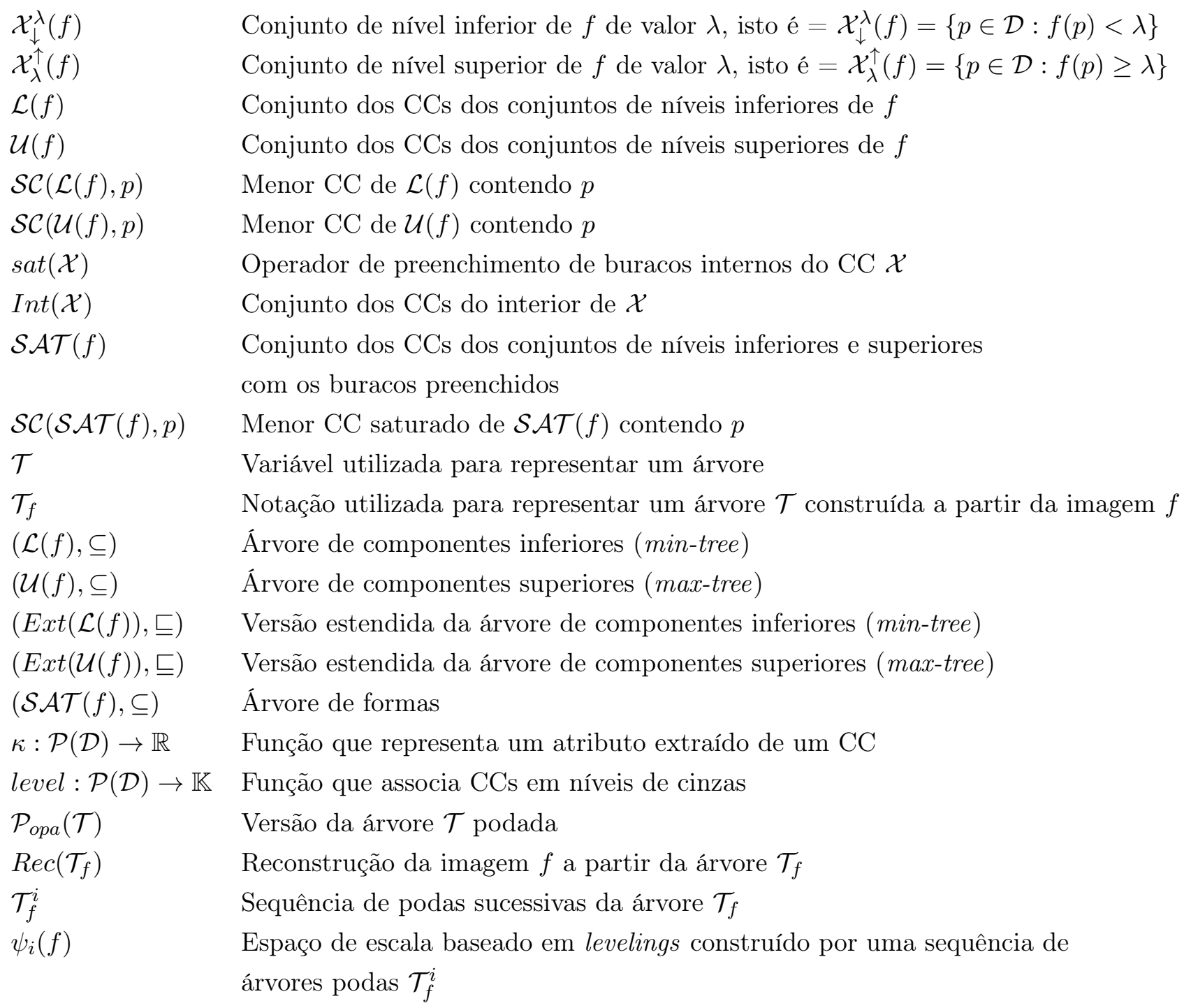

\section{Ültimos levelings}

$r^{+}$e $r^{-} \quad$ Notação utilizadas para representar os operadores residual positivo e negativo

$\theta \quad$ Notação utilizada para representar o operador última abertura

$\mathcal{R}_{\theta}^{+}$e $\mathcal{R}_{\theta}^{-} \quad$ Notação utilizada para representar os operadores residuais estendidos positivo e negativo

$\mathcal{R}_{\theta}(f) \quad$ Notação utilizada para representar um operador último leveling

$\mathcal{I} \subset \mathbb{N} \quad$ Conjunto de índices de uma família de primitivas

$q_{\mathcal{I}}$ e $q_{\kappa} \quad$ Funções associadas ao índice $q_{\mathcal{I}}$ e ao atributo $q_{\kappa}$

$\mathcal{N} r(i) \quad$ Conjunto de vértices removidos da árvore $\mathcal{T}_{f}^{i}$ para construir a versão podada $\mathcal{T}_{f}^{i+1}$

$r_{\mathcal{T}_{f}^{i}}^{+}(C) \quad$ Notação utilizada para representar o valor residual dos pixels do CC $C$ da árvore $\mathcal{T}_{f}^{i}$

$\Gamma \quad$ Função booleana sobre os vértices de uma árvore utilizada para decidir se um vértice é podado ou não

$\Omega \quad$ Função booleana sobre os vértices de uma árvore utilizada para decidir se um vértice é filtrado ou não

$T_{\kappa} \quad$ Conjunto de limiares para o atributo crescente $\kappa$

$\Delta \quad$ Variável utilizada para representar um parâmetro das estratégias de podas: transições graduais e mser 


\section{Lista de Algoritmos}

4.1 Algoritmo genérico baseado em union-find para construção da árvore de componentes. 38

4.2 Ordenação dos pixels das zonas planas de uma imagem pelos seus níveis de cinza. . 40

4.3 Construção da árvore de componentes por union-find . . . . . . . . . . . . . . . 41

4.4 Algoritmo para construção da imagem interpolada. . . . . . . . . . . . . . . . . . . 46

4.5 Algoritmos para construção da árvore de formas proposto por Géraud et al. (2013). 47

5.1 Computação da reconstrução por dilatação a partir da max-tree estendida. ..... 61

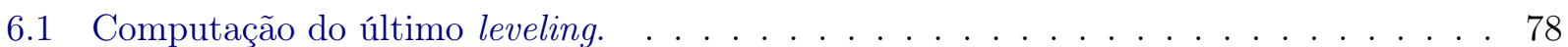

6.2 Computação do último leveling com informações associadas. . . . . . . . . . . . . . 81

7.1 Computação básica de uma estratégia de escolha da família de primitivas. . . . . . . . 85

7.2 Seleção de vértices baseadas em valores de extinção para as árvores max-tree, min-tree ou de formas. . . . . . . . . . . . . . . . . . . . . . . . 88

7.3 Algoritmo genérico para computar descritores computáveis incrementalmente. . . . . . 92

7.4 Computação incremental do atributo área. . . . . . . . . . . . . . . . . . . . 93

7.5 Extração dos vértices MSER de uma árvore max-tree, min-tree ou árvore de formas. . 95 


\section{Lista de Figuras}

1.1 Exemplo do operador residual gradiente aplicado em uma imagem. . . . . . . . . . . 2

1.2 Exemplo da extração de resíduos com o operador esqueleto por discos maximais. $\quad$. 3

1.3 Exemplo da extração de contraste com o operador última abertura . . . . . . . . . . 4

2.1 Exemplos de relações de adjacências . . . . . . . . . . . . . . . . . . . . . . . . . . . 10

2.2 Exemplo de extração de componentes conexos . . . . . . . . . . . . . . . . . . . . . . 10

2.3 Exemplo de extração de zonas planas . . . . . . . . . . . . . . . . . . . . . . . . . . . 11

2.4 Exemplo de perfil de intensidade . . . . . . . . . . . . . . . . . . . . . . . . . . . . . 11

2.5 Exemplo da aplicação de erosão e dilatação com EE formato de cruz . . . . . . . . . 13

2.6 Exemplo da aplicação de abertura e fechamento com EE formato de cruz . . . . . . . 14

2.7 Exemplo de reconstrução por dilatação para conjuntos . . . . . . . . . . . . . . . . . 15

2.8 Exemplo de reconstrução por erosão para conjuntos . . . . . . . . . . . . . . . . . . . 15

2.9 Exemplo de reconstrução por dilatação para imagens . . . . . . . . . . . . . . . . . . 15

2.10 Exemplo de reconstrução por erosão para imagens . . . . . . . . . . . . . . . . . . . 16

2.11 Exemplos de aberturas e fechamentos por atributos. . . . . . . . . . . . . . . . . 16

2.12 Exemplos de aberturas e fechamentos por atributos. . . . . . . . . . . . . . . . . . . 17

3.1 Exemplo de aplicações de operadores para fins de filtragem. . . . . . . . . . . . . . . 19

3.2 Exemplos de partições de operadores conexos . . . . . . . . . . . . . . . . . . . . . . 20

3.3 Exemplos de aplicações de operadores levelings . . . . . . . . . . . . . . . . . . . . . 21

3.4 Exemplos de aplicações de operadores monotone planing . . . . . . . . . . . . . . . . 22

3.5 Ilustração destacando que os operadores monotone planing não criam novas extremas regionais. . . . . . . . . . . . . . . . . . . . . . . . . . 24

3.6 Exemplos de construções de levelings a partir de imagens marcadoras. . . . . . . . . 27

3.7 Exemplos de imagens de um espaço de escala baseado em levelings . . . . . . . . . . 28

4.1 Representação de uma imagem por meio de árvore. . . . . . . . . . . . . . . . . . . . . 29

4.2 Exemplo de um diagrama de Hasse . . . . . . . . . . . . . . . . . . . . . . . . . . . . 30

4.3 Exemplo de um diagrama de Hasse que não é uma árvore . . . . . . . . . . . . . . . 30

4.4 Exemplo de um diagrama de Hasse que é uma árvore . . . . . . . . . . . . . . . . . . 31

4.5 Exemplo de operação de poda para uma dada árvore. . . . . . . . . . . . . . . . . . . 32

4.6 Exemplo de conjuntos de níveis extraídos de uma imagem . . . . . . . . . . . . . . . 33

4.7 Exemplos de árvores de componentes. . . . . . . . . . . . . . . . . . . . . . . . . . . 35

4.8 Exemplos de árvores de componentes destacando os menores CCs . . . . . . . . . . . 36

4.9 Exemplos de construções de árvores de componentes estendidas. . . . . . . . . . . . . 37 
4.10 Árvore de decisão para auxiliar a escolha de um algoritmo para construção da árvore de componentes . . . . . . . . . . . . . . . . . . . . 38

4.11 Exemplo de computação da min-tree . . . . . . . . . . . . . . . . . . . . . . 39

4.12 Exemplo de árvore de formas . . . . . . . . . . . . . . . . . . . . . 41

4.13 Exemplo mostrando um CC $A$ com seu conjunto $\operatorname{sat}(A)$ e $\operatorname{Ext}(A)$. . . . . . . . . . 42

4.14 Exemplo de interpolação de imagens baseada em funções valor-conjunto . . . . . . . 43

4.15 Exemplo de grade Khalimsky . . . . . . . . . . . . . . . . . . . . . 44

4.16 Exemplo das operações estrela e fechamento . . . . . . . . . . . . . . . . 45

4.17 Exemplos de planos cartesiano dos conjuntos $\mathbb{Z}^{2}, H^{2}$ e $\frac{1}{2} H^{2} \ldots \ldots \ldots \ldots$

4.18 Exemplo de interpolação . . . . . . . . . . . . . . . . . . . . . 46

4.19 Exemplo de construção da árvore de formas. . . . . . . . . . . . . . . . . . . . 48

4.20 Exemplos das operações de podas e reconstruções. . . . . . . . . . . . . . . . . . . 49

5.1 Ilustrações das configurações de podas em uma árvore max-tree. . . . . . . . . . . . 55

5.2 Ilustração do enunciado da Proposição 5.6 . . . . . . . . . . . . . . . . . . 57

5.3 Ilustrações das configurações de podas. . . . . . . . . . . . . . . . . . . . 58

5.4 Ilustração destacando o passo inicial da computação da reconstrução de árvores podas a partir de imagens marcadoras . . . . . . . . . . . . . . . . . . . 60

5.5 Ilustração destacando o passo final da computação da reconstrução de árvores podas a partir de imagens marcadoras . . . . . . . . . . . . . . . . . 6 60

5.6 Comparação dos tempos computacionais de algoritmos para reconstrução por dilatação 62

5.7 Comparação dos tempos computacionais de algoritmos para reconstrução por dilatação 62

5.8 Exemplo de um espaço de escala gerado a partir de uma max-tree. . . . . . . . . . 65

5.9 Exemplos de imagens de um espaço de escala de levelings obtidos por podas pelo atributo de área . . . . . . . . . . . . . . . . . . . . . . . . 66

5.10 Exemplos de imagens de espaços de escalas de levelings associados com famílias de marcadores. . . . . . . . . . . . . . . . . . . . 67

6.1 Um exemplo simples de computação da última abertura por atributo . . . . . . . . . 70

6.2 Exemplos de aplicações de últimos levelings . . . . . . . . . . . . . . . . . 71

6.3 Ilustração da classe dos operadores últimos levelings. . . . . . . . . . . . . . . . . 72

6.4 Um exemplo de computação do último fechamento por atributo . . . . . . . . . . . 76

6.5 Exemplo de computação de último leveling utilizando uma árvore de componentes $\quad 79$

6.6 Exemplo de computação de último leveling com funções associadas utilizando uma árvore de componentes . . . . . . . . . . . . . . . . . . . . . . . 82

6.7 Exemplos de aplicações de últimos levelings . . . . . . . . . . . . . . . . . . 82

7.1 Exemplo de computação de um último leveling negativo onde é evidenciado que a ordem das marcações nos vértices da árvore não modifica o resultado final dos últimos

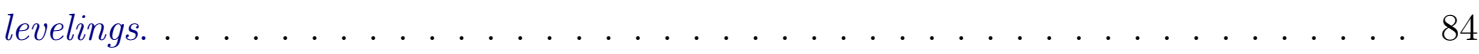

7.2 Análise da evolução residual para o pixel marcado de vermelho da imagem de entrada. 86

7.3 Análise da evolução residual para o pixel marcado de vermelho da imagem de entrada. 87

7.4 Análise da evolução residual para o pixel marcado de vermelho da imagem de entrada. 89 
7.5 Exemplos de entrada e saída dos processamentos baseados em transições graduais e valores de extinção. . . . . . . . . . . . . . . . . . . . . . . . . . . . . . . . . . . . . . 89

7.6 Esquema para filtrar os $i$-ésimos resíduos $r_{i}$ extraídos de regiões indesejáveis. . . . . 90

7.7 Aplicações dos últimos filtros por grãos com informação de formas circulares. . . . . 93

7.8 Aplicação da última abertura com informação de formas de estruturas finas. . . . . . 93

7.9 Aplicações dos últimos filtros por grãos com filtragem de resíduos indesejáveis baseadas em MSER. . . . . . . . . . . . . . . . . . . . . . . . . . . . 96

7.10 Aplicações dos últimos filtros por grãos com filtragem de resíduos indesejáveis baseada em uma composição de filtragens . . . . . . . . . . . . . . . . . . . . . . . . . . . 97

8.1 Exemplo de imagem de fundo de olho e sua rede de vasos sanguíneos segmentada manualmente. . . . . . . . . . . . . . . . . . . . . . . . . . 101

8.2 Exemplo de uma imagem colorida de retina. . . . . . . . . . . . . . . . . . . . . . . . 102

8.3 Exemplo de imagem de fundo de olho e sua rede de vasos sanguíneos. . . . . . . . . . 102

8.4 Exemplo de alongamento de vasos sanguíneos segmentados de imagens de fundo de olho. . . . . . . . . . . . . . . . . . . . . . . . . . 103

8.5 Ilustração mostrando os passos seguidos pelo método proposto para segmentação de vasos sanguíneos em imagens de retinas . . . . . . . . . . . . . . . . . . . . . . . . . 104

8.6 Resultado de quatro segmentações de vasos sanguíneos das 20 imagens testadas do banco DRIVE . . . . . . . . . . . . . . . . . . . . . . . . . . 105

8.7 Resultado de quatro segmentações de vasos sanguíneos das 20 imagens testadas do banco STARE . . . . . . . . . . . . . . . . . . . . . . . 105

9.1 Exemplos de imagens de documentos históricos extraídas dos bancos dos concursos DIBCOs. . . . . . . . . . . . . . . . . . . . . . . . . . . 107

9.2 Exemplos de aplicações de fechamentos top-hats em imagens de documentos históricos. 108

9.3 Exemplos de imagens de documentos históricos dos bancos dos concursos DIBCOs destacando em vermelho as regiões maximalmente estáveis. . . . . . . . . . . . . . . 109

9.4 Resultados de quatro binarizações de imagens de documentos históricos . . . . . . . 110

10.1 Imagens de cena do banco de imagens ICDAR (Lucas et al., 2003) . . . . . . . . . 113

10.2 Visão geral do método proposto. . . . . . . . . . . . . . . . . . . . . . . . . . . . . . 114

10.3 Extração das regiões de texto candidatas. . . . . . . . . . . . . . . . . . . . . . . . . 116

10.4 Resultados da classificação de regiões de texto usando o T-HOG. . . . . . . . . . . . 117

10.5 Exemplos de imagens da base ICDAR. . . . . . . . . . . . . . . . . . . . . . . . 118 


\section{Lista de Tabelas}

8.1 Tabela comparativa de diferentes métodos para segmentação de vasos sanguíneos nas imagens do banco DRIVE. . . . . . . . . . . . . . . . . . . . 106

8.2 Tabela comparativa de diferentes métodos para segmentação de vasos sanguíneos nas imagens do banco STARE. . . . . . . . . . . . . . . . . . . . . 106

9.1 Tabela comparativa com resultados obtidos com as imagens de documentos impressos.111

9.2 Tabela comparativa com resultados obtidos com as imagens de documentos escritos à mão. . . . . . . . . . . . . . . . . . . . . . . . . . . . . . . . . . . 112

10.1 Tabela comparativa de diferentes métodos para localização de textos. . . . . . . . . . 119 


\subsection{Considerações preliminares}

Nas últimas décadas, os esforços em pesquisas envolvendo imagens digitais têm sido crescente, possibilitando o desenvolvimento de inúmeras teorias e tecnologias em diversos segmentos da ciência e indústria. Neste cenário, surgiu a Morfologia Matemática (MM) entre os anos de 1960 e 1969, inicialmente desenvolvida por Georges Matheron e Jean Serra na Escola de Minas de Paris, França. A MM tornou-se, com o passar dos anos, uma importante ferramenta para análise e processamento de imagens. Seu domínio de atuação cobre um largo espectro de aplicações em análise e processamento de imagens, que vai desde o tratamento de imagens microscópicas até o processamento de imagens de satélites, passando por aplicações industriais, médicas, automação de escritório, entre outras (Dougherty e Lotufo, 2003; Najman e Talbot, 2013; Serra, 1982; Soille, 2003).

A MM é uma teoria matemática abrangente e bastante empregada em problemas de análise e processamento de imagens, onde as transformações de imagens (em MM chamadas de operadores de imagens) são vistas como mapeamentos entre reticulados completos (Banon e Barrera, 1994; Heijmans, 1994; Najman e Talbot, 2013; Serra, 1982). Em poucas palavras, um reticulado completo é uma estrutura algébrica composta por um conjunto equipado por uma relação de ordem (parcial ou total) tal que qualquer subconjunto deste conjunto possui um ínfimo e um supremo no conjunto (Heijmans, 1994; Serra, 1982). Basicamente, existem dois reticulados completos amplamente úteis para trabalhar com problema de análise e processamento de imagens, são eles: o reticulado dos subconjuntos $\mathcal{D} \subset \mathbb{Z}^{2}$, e o reticulado das imagens (ou funções) definidas com o domínio $\mathcal{D}$ e o contradomínio $\mathbb{K}=\{0,1, \ldots, K\} \subset \mathbb{N}$. Mais precisamente:

- Reticulado dos subconjuntos: é a 4-upla $(\mathcal{P}(\mathcal{D}), \subseteq, \cap, \cup)$, onde: $(1) \mathcal{P}(\mathcal{D})$ é o conjunto de todos os subconjuntos de $\mathcal{D} ;(2) \subseteq$ é a usual relação de ordem inclusão sobre $\mathcal{P}(\mathcal{D}) ;(3) \cap$ é o ínfimo definido pela usual operação de intersecção da teoria dos conjuntos; (4) $\cup$ é o supremo definido pela usual operação de união da teoria dos conjuntos.

- Reticulado das imagens: é a 4-upla $(\mathcal{F}(\mathcal{D}), \leq, \wedge, \vee)$, onde: $(1) \mathcal{F}(\mathcal{D})$ é o conjunto de todas as imagens $f: \mathcal{D} \rightarrow \mathbb{K} ;(2) \leq$ é uma relação de ordem sobre $\mathcal{F}(\mathcal{D})$ definida da seguinte forma: $\forall f, g \in \mathcal{F}(\mathcal{D}), f \leq g \Longleftrightarrow \forall p \in \mathcal{D}, f(p) \leq g(p)$; (3) $\wedge$ é o ínfimo definido pelo operador mínimo pontual, ou seja, $\forall f, g \in \mathcal{F}(\mathcal{D}), f \wedge g \Longleftrightarrow \forall p \in \mathcal{D},[f \wedge g](p)=\min \{f(p), g(p)\} ;(4)$ $\vee$ é o supremo definido pelo operador máximo pontual, isto é, $\forall f, g \in \mathcal{F}(\mathcal{D}), f \vee g \Longleftrightarrow \forall p \in$ $\mathcal{D},[f \vee g](p)=\max \{f(p), g(p)\}$.

Assim, chama-se de operador de subconjuntos um mapeamento sobre o reticulado dos subconjuntos, ou seja, $\Psi: \mathcal{P}(\mathcal{D}) \rightarrow \mathcal{P}(\mathcal{D})$. Analogamente, chama-se de operador de imagens um mapeamento 
sobre o reticulado das imagens, isto é, $\psi: \mathcal{F}(\mathcal{D}) \rightarrow \mathcal{F}(\mathcal{D})$. Em particular, quando um operador de imagens (respectivamente, subconjuntos) satisfaz as propriedades de ser $(i)$ crescente e $(i i)$ idempotente, ele é chamado de filtro morfológico (Serra, 1988). A primeira condição é para preservar a relação de ordem do reticulado e a segunda condição corresponde ao fato de que operações crescentes não são reversíveis, e isso nos faz de fato esperar uma redução no conteúdo da informação após a aplicação do operador (Serra e Vincent, 1992). A chave para o sucesso com filtros morfológicos é entender este fato, e usando esta característica é possível destruir gradualmente o conteúdo indesejável da imagem como o ruído e a irregularidade do fundo, preservando ao mesmo tempo o conteúdo desejado o máximo possível (Najman e Talbot, 2013). Algumas vezes é necessário destruir o conteúdo indesejável em uma imagem, mas na prática não é tão fácil assim. Neste caso, uma estratégia complementar é eliminar efetivamente o conteúdo desejável da imagem, mas restaurá-lo por meio de uma diferença (ou subtração) com a imagem original. Isto dá origem à ideia dos operadores residuais. De uma maneira simples, os operadores residuais são transformações que envolvem combinações de operadores morfológicos com subtrações (Alves e Hashimoto, 2014; Beucher, 2007; Dougherty e Lotufo, 2003; Hernandez e Marcotegui, 2011; Leite e Guimarães, 2001; Najman e Talbot, 2013; Serra, 1982; Soille, 2003).

Mais precisamente (veja a Definição 1.1), um operador residual $r$ é definido pela diferença (pixel a pixel) entre dois operadores, digamos $\psi$ e $\phi$ (respectivamente, $\Psi$ e $\Phi)$, aplicados a uma dada imagem $f \in \mathcal{F}(\mathcal{D})$ (respectivamente, subconjunto $X \in \mathcal{P}(\mathcal{D})$ ), isto é, $r(f)=\psi(f)-\phi(f)$ (respectivamente, $r(X)=\Psi(X) \backslash \Phi(X)$ ). Além do mais, chama-se $\psi$ e $\phi$ (respectivamente, $\Psi$ e $\Phi)$ de primitivas e para um pixel $p \in \mathcal{D}$ diz-se que, se $[\psi(f)](p)>[\phi(f)](p)$ (respectivamente, $p \in \Psi(X)$ e $p \notin \Phi(X))$ então $p$ possui um resíduo positivo em $r$, senão se $[\psi(f)](p)<[\phi(f)](p)$ (respectivamente, $p \notin \Psi(X)$ e $p \in \Phi(X))$ então $p$ possui um resíduo negativo em $r$, caso contrário, $p$ possui um resíduo nulo em $r$. Desta forma, diversos operadores residuais foram propostos ao longo das últimas décadas (Alves e Hashimoto, 2014; Beucher, 1994, 2007; Hernandez e Marcotegui, 2011; Marcotegui et al., 2011; Serra, 1982) e são amplamente utilizados em problemas de análise e processamento de imagens, como por exemplo: o gradiente morfológico (ver exemplo na Figura 1.1), definido como os resíduos obtidos entre os operadores dilatação e erosão, ou a abertura top-hat, definido como os resíduos obtidos entre os operadores identidade e a abertura.

Definição 1.1 (Operador residual). Um operador residual $r$ é a diferença entre dois operadores, digamos $\psi$ e $\phi$ (respectivamente, $\Psi$ e $\Phi$ ) aplicados a uma dada imagem $f \in \mathcal{F}(\mathcal{D})$ (respectivamente, subconjunto $X \in \mathcal{P}(\mathcal{D}))$, ou seja, $\forall p \in \mathcal{D},[r(f)](p)=[\psi(f)](p)-[\phi(f)](p)$ (respectivamente, $r(X)=\Psi(X) \backslash \Phi(X))$.

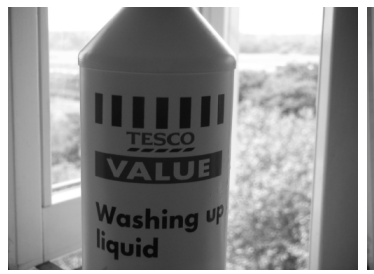

imagem de entrada $f$

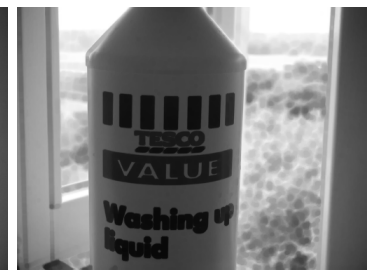

erosão $\varepsilon_{B}(f)$

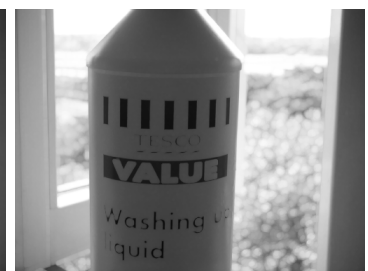

dilatação $\delta_{B}(f)$

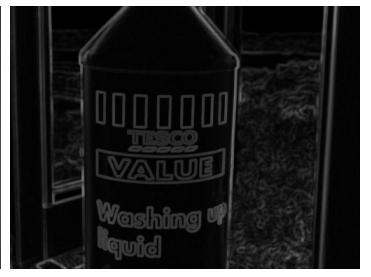

resíduo $r(f)=\delta(f)-\varepsilon(f)$

Figura 1.1: Exemplo do operador residual gradiente aplicado em uma imagem.

Os operadores residuais têm sido estendidos para extraírem resíduos de duas famílias de operadores de imagens $\left\{\psi_{i}: i \in \mathcal{I}\right\}$ e $\left\{\phi_{i}: i \in \mathcal{I}\right\}$ (respectivamente, subconjuntos $\left\{\Psi_{i}: i \in \mathcal{I}\right\}$ e $\left\{\Phi_{i}: i \in \mathcal{I}\right\}$ ) 
indexados por um conjunto de índices $\mathcal{I}=\left\{0,1, \ldots, \mathcal{I}_{M A X}\right\}$, de tal modo que o $i$-ésimo operador residual $r_{i}$ é definido pelos resíduos extraídos das $i$-ésimas primitivas, isto é, $f \in \mathcal{F}(\mathcal{D}), r_{i}(f)=$ $\psi_{i}(f)-\phi_{i}(f)$ (respectivamente, $\left.X \in \mathcal{P}(\mathcal{D}), r(X)=\Psi_{i}(f) \backslash \Phi_{i}(f)\right)$ e um operador residual estendido $\mathcal{R}_{\theta}$ é definido como o supremo dos operadores residuais $r_{i}$ extraídos da família de primitivas, ou seja, $\mathcal{R}_{\theta}(f)=\sup _{i \in \mathcal{I}}\left\{r_{i}(f)\right\}$ (respectivamente, $\mathcal{R}_{\theta}(X)=\sup _{i \in \mathcal{I}}\left\{r_{i}(X)\right\}=\bigcup_{i \in \mathcal{I}}\left\{r_{i}(X)\right\}$ ). Neste contexto, um operador para subconjuntos extremamente utilizado em análise de imagens é o esqueleto por discos maximais, ilustrado na Figura 1.2, que é definido como a união (supremo) dos resíduos extraídos de famílias de erosões $\left\{\varepsilon_{i}: i \in \mathcal{I}\right\}$ e aberturas de erosões $\left\{\gamma_{\mathcal{B}} \varepsilon_{i}: i \in \mathcal{I}\right\}$, ou seja, $S K(X)=\bigcup_{i \in \mathcal{I}}\left\{r_{i}(X): r_{i}(X)=\varepsilon_{i}(X) \backslash \gamma_{\mathcal{B}}\left(\varepsilon_{i}(X)\right)\right\}$ (Beucher, 1994).

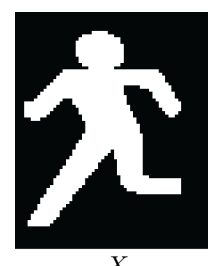

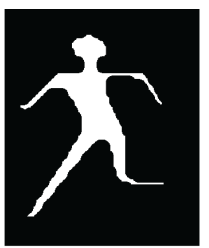
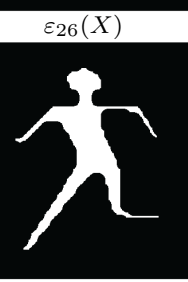

$\gamma\left(\varepsilon_{26+1}(X)\right)$

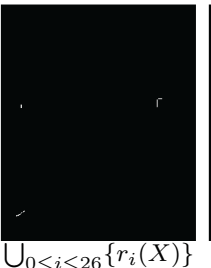

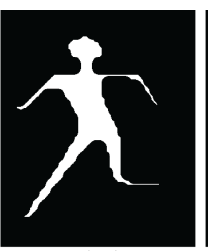

$\varepsilon_{28}(X)$

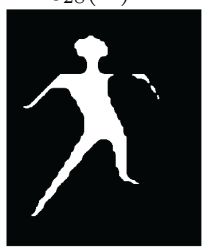

$\gamma\left(\varepsilon_{28+1}(X)\right)$

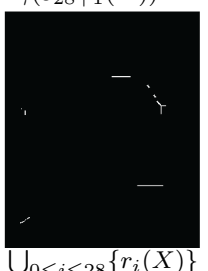

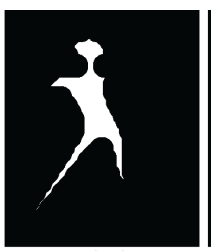

$\varepsilon_{38}(X)$
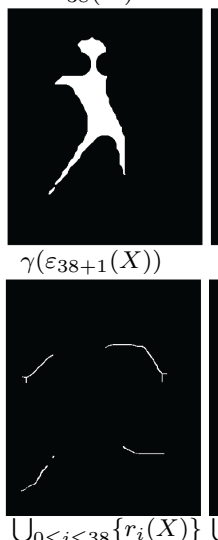

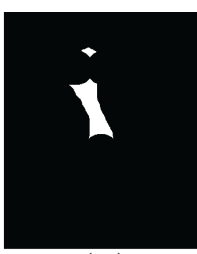

$\varepsilon_{58}(X)$

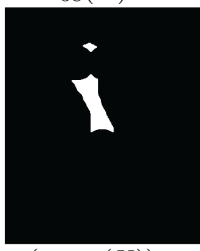

$\gamma\left(\varepsilon_{58+1}(X)\right)$

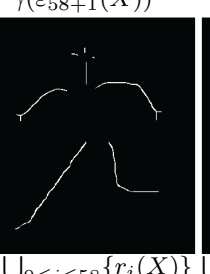

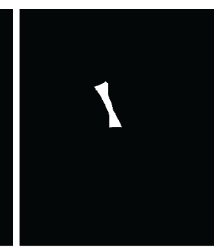

$\varepsilon_{74}(X)$

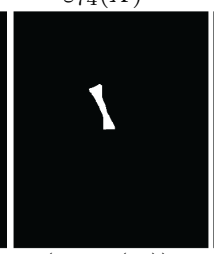

$\gamma\left(\varepsilon_{74+1}(X)\right) \quad \gamma\left(\varepsilon_{94+1}(X)\right)$

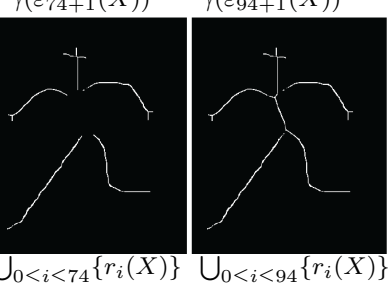

Figura 1.2: Exemplo da extração de resíduos com o operador esqueleto por discos maximais.

Também nesta direção, Beucher (2007) propôs o operador de imagens última abertura, onde as primitivas são aberturas consecutivas, ou seja, $\psi_{i}=\gamma_{i}$ e $\phi_{i}=\gamma_{i+1}$, de uma família crescente de aberturas $\left\{\gamma_{i}: i \in \mathcal{I}\right\}$ e assim definida por $\mathcal{R}_{\theta}(f)=\sup _{i \in \mathcal{I}}\left\{r_{i}(f): r_{i}(f)=\gamma_{i}(f)-\gamma_{i+1}(f)\right\}$. Na Figura 1.3 é apresentado um exemplo de aplicação da última abertura para extração de contraste.

A última abertura é um operador residual não-paramétrico e multi-escala definido sobre um espaço de escala baseado em aberturas $\left(\gamma_{0}, \gamma_{1}, \ldots, \gamma_{\mathcal{I}_{M A X}}\right)$ de forma que os resíduos $\left(r_{0}, r_{1}, \ldots, r_{\mathcal{I}_{M A X-1}}\right)$ são extraídos por primitivas consecutivas deste espaço de escala. Desta forma, objetos contrastados de uma imagem de entrada são detectados quando são filtrados por um desses operadores, gerando assim um resíduo $r_{i}$ extraído da $i$-ésima abertura. Esta maneira de extrair resíduos proposto por Beucher (2007) também foi utilizada mais tarde em outros trabalhos (Alves e Hashimoto , 2014; Alves et al., 2013; Hernandez e Marcotegui, 2011; Meyer, 2010; Retornaz e Marcotegui , 2007) para extrair resíduos de espaços de escalas baseados em operadores conexos. Em poucas palavras, um operador conexo é uma transformação que não cria novos contornos (ou bordas) na imagem (Salembier e Serra, 1995). Primeiramente, Retornaz e Marcotegui (2007) propuseram a última abertura por atributo, uma extensão da última abertura introduzida por Beucher (2007), onde as primitivas são aberturas por atributos e mais adiante, Fabrizio e Marcotegui (2009) desenvolveram um algoritmo eficiente utilizando uma estrutura de dados chamada max-tree e, 
Família de abertura contruída por elementos estruturantes discos com raios crescentes

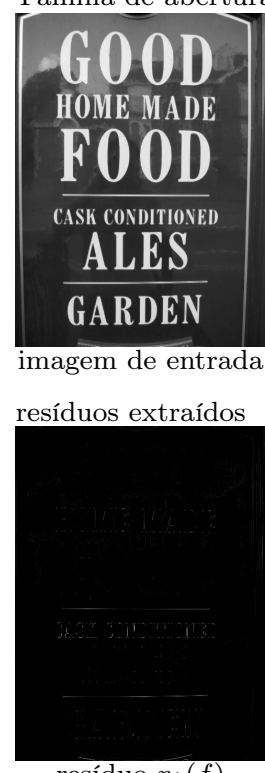

resíduo $r_{1}(f)$

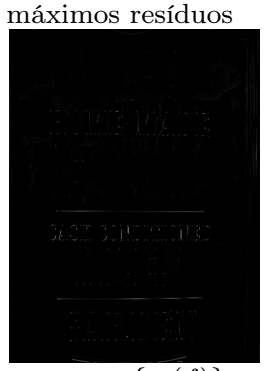

$\sup _{i \leq 1}\left\{r_{i}(f)\right\}$
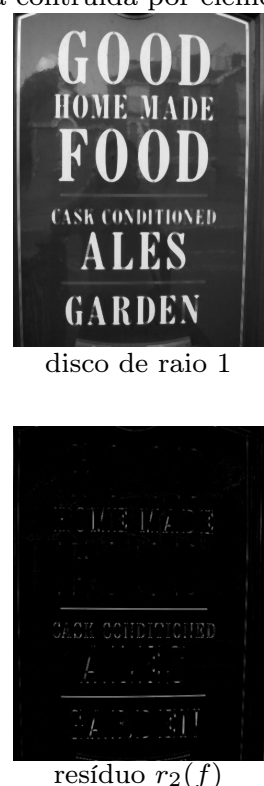

resíduo $r_{2}(f)$

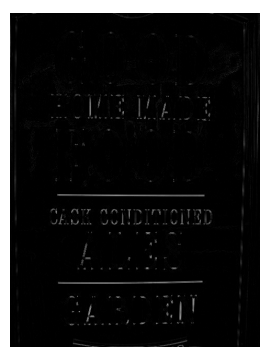

$\sup _{i \leq 2}\left\{r_{i}(f)\right\}$

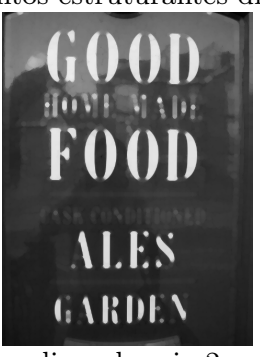

disco de raio 2

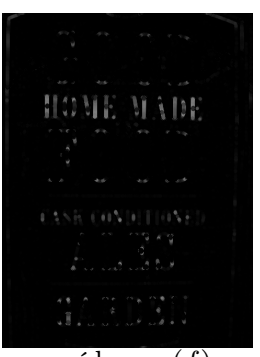

resíduo $r_{4}(f)$

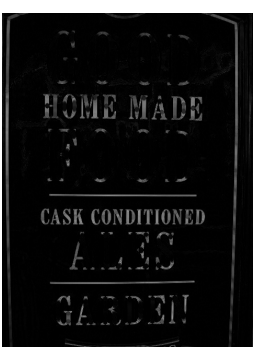

$\sup _{i \leq 4}\left\{r_{i}(f)\right\}$

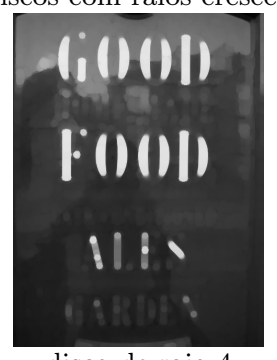

disco de raio 4

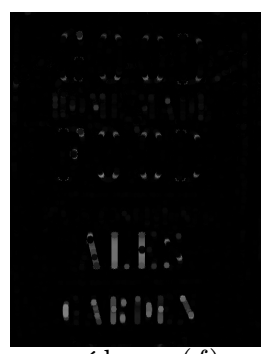

resíduo $r_{6}(f)$

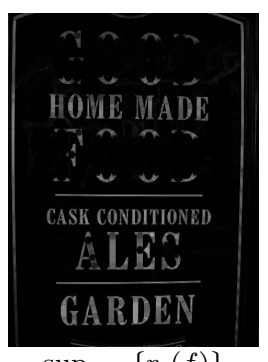

$\sup _{i \leq 6}\left\{r_{i}(f)\right\}$
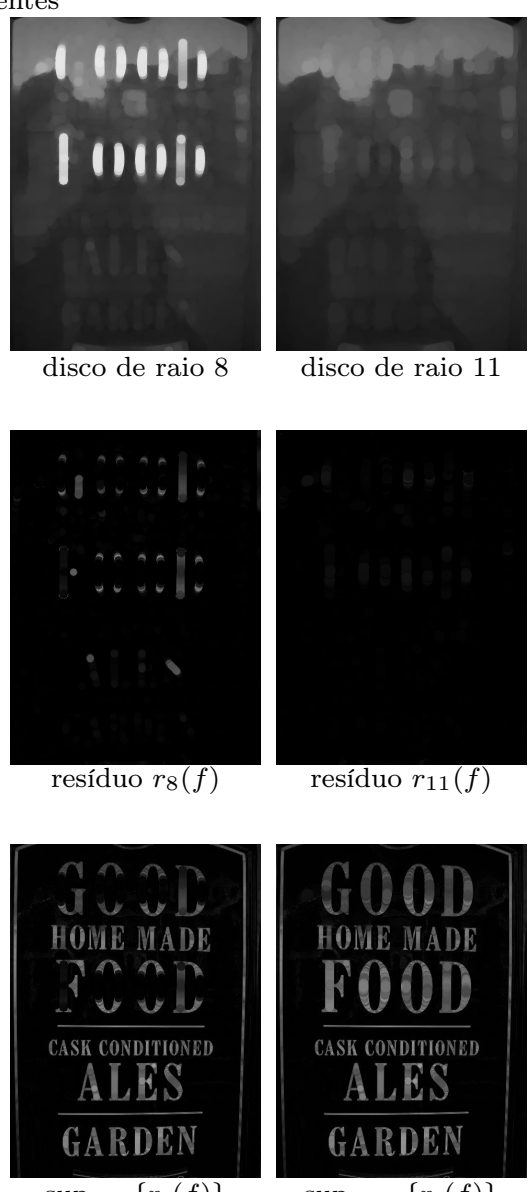

$\sup _{i \leq 11}\left\{r_{i}(f)\right\}$

Figura 1.3: Exemplo da extração de contraste com o operador última abertura.

Hernandez e Marcotegui (2011) utilizaram informações de formas no processo de extração de resíduos da última abertura por atributo. Diferentemente desses trabalhos, Meyer (2010) e depois Alves e Hashimoto (2014), propuseram extrair resíduos de primitivas consecutivas de um espaço de escala baseado em uma larga classe de operadores conexos chamada levelings. Os levelings utilizados nesses trabalhos podem gerar resíduos positivos e negativos, o que não acontece com a última abertura por atributo. Neste caso, trata-se separadamente o supremo dos resíduos positivos e dos negativos, e depois os combinam (Alves e Hashimoto, 2014; Alves et al., 2013; Meyer, 2010).

\subsection{Objetivos}

A presente tese está centrada em um estudo sobre uma classe de operadores residuais estendidos definidos por meio de espaços de escalas baseados em levelings. Um operador desta classe de operadores residuais analisa a evolução dos valores residuais presentes em dois operadores consecutivos de um espaço de escala e os maiores valores residuais são considerados. Os valores residuais revelam uma importante informação sobre o contraste de uma imagem. Além dos valores residuais, outras informações associadas com eles podem ser obtidas no momento da extração residual, tais como propriedades das regiões que produziram os valores residuais. Alguns exemplos de operadores dessa classe conhecidos na literatura, incluem a última abertura (respectivamente, fechamento) por atributos (Retornaz e Marcotegui, 2007) e o último filtro por grãos (Alves e Hashimoto, 2014). Eles têm sido usados com sucesso em uma etapa de pré-processamento em diversas aplicações, como por exem- 
plo, a localização de texto em imagens de cenas (Alves e Hashimoto, 2010; Retornaz e Marcotegui , 2007), a segmentação de fachadas de edifícios (Hernandez e Marcotegui, 2011), a restauração de imagens de documentos históricos (Meyer, 2010). Com base nessas considerações, apresenta-se neste trabalho a classe de operadores residuais denominada últimos levelings, na qual destacamos suas propriedades, relações com operadores conhecidos na literatura, definições de novos operadores residuais, algoritmos eficientes para implementação desses operadores e aplicações desta abordagem em problemas de análise e processamento de imagens.

\subsection{Principais contribuições}

As principais contribuições deste trabalho são as seguintes:

- Os últimos levelings consistem em uma classe de operadores residuais definidos por meio de espaços de escalas baseados em levelings. Neste trabalho, um espaço de escala baseado em levelings é representado por meio de uma hierarquia construída por conjuntos de níveis, ou seja, árvore de componentes ou árvore de formas. Primeiramente, dada uma imagem $f \in$ $\mathcal{F}(\mathcal{D})$ constrói-se uma árvore $\mathcal{T}_{f}$ que representa esta imagem $f$. Como contribuição original, é mostrado que determinadas operações sobre esta árvore resulta em um operador leveling da imagem $f$ e mais, é demostrado ainda que esta árvore é capaz de produzir uma família de levelings que satisfazem propriedades de espaço de escala de levelings.

- A classe dos últimos levelings é uma outra importante contribuição deste trabalho, onde são estabelecidos: $(i)$ propriedades, $(i i)$ algoritmos eficientes para implementação de últimos levelings, (iii) estratégias para a escolha de uma família de primitivas e (iv) estratégias de filtragens de resíduos indesejáveis.

- Por fim, são apresentadas aplicações práticas dos últimos levelings e ainda são disponibilizados plugins para o popular software livre Image ${ }^{1}$, que dão acesso a alguns operadores últimos levelings. Além do acesso aos operadores, os plugins contêm funcionalidades que auxiliam um pesquisador na solução de um problema por meio de abordagem residual. Instruções para download e instalação dos plugins podem ser encontradas no website http://www.ime.usp.br/ wonder/plugins.

\subsection{Organização do trabalho}

O restante deste trabalho está organizado em três partes, compreendendo: conceitos, metodologias e aplicações. A primeira parte da tese é dedicada às apresentações de conceitos, notações, algoritmos e propriedades relacionadas com: imagens e operadores morfológicos (Capítulo 2); operadores conexos e suas especializações como os levelings e os monotone planing (Capítulo 3); representações hierárquicas de conjuntos de níveis, incluindo árvores de componentes e árvores de formas (Capítulo 4). A segunda parte é dedicada às principais contribuições originais desta pesquisa, as quais incluem: $(i)$ uma representação compacta e eficiente de um espaço de escalas baseado em levelings por meio de árvores (Capítulo 5); (ii) apresentação da classe dos últimos levelings, isto é, definições, conceitos, algoritmos, propriedades e relações com operadores conhecidos na literatura (Capítulo 6); (iii) estratégias para construção de operadores últimos levelings (Capítulo 7). A terceira parte é dedicada as aplicações dos últimos levelings em problemas de análise e processamento

\footnotetext{
${ }^{1}$ ImageJ é um software livre, desenvolvido em Java, para análise e processamento de imagens. Ele pode ser encontrado gratuitamente pelo website http://rsb.info.nih.gov/ij/
} 
de imagens, incluindo: a segmentação de vasos sanguíneos em imagens de retinas (Capítulo 8), a binarização de texto em imagens de documentos históricos (Capítulo 9) e a localização de textos em imagens de cenas (Capítulo 10). Por fim, no Capítulo 11 são apresentadas as conclusões e trabalhos futuros. 


\section{Parte I}

\section{Conceitos sobre imagens, operadores e representações}

\section{Resumo}

Esta primeira parte da tese é dedicada a apresentações de conceitos, notações, algoritmos e propriedades relacionados com:

1. Imagens, como por exemplo: definições e notações de componentes conexos, zonas planas, extremas regionais, partições sobre dominio da imagem e perfis de intensidade;

2. Operadores morfológicos, como por exemplo: propriedades relacionadas aos operadores morfológicos e, definições e notações sobre elemento estruturante, erosão, dilatação, abertura, fechamento, reconstrução por dilatação e reconstrução por erosão;

3. Operadores conexos e suas especializaçôes como os levelings e os monotone planing, incluindo: definições, notações, propriedades relacionados a estes operadores e construção de espaço de escala baseado em levelings;

4. Representações hierarquias de conjuntos de níveis, incluindo: definições, notações, algoritmos e propriedades relacionadas as árvores construídas a partir de conjuntos parcialmente ordenados, como por exemplo: árvores de componentes e árvores de formas.

Vale salientar, que todos os conceitos, notações, algoritmos e propriedades apresentadas nesta primeira parte da tese são úteis para o entendimento dos conceitos discutidos nas duas próximas partes desta tese e mais, também são assumidas definições usuais de conjuntos, relações, funções, grafos, conjuntos ordenados, reticulados e espaços topológicos que podem ser encontradas em um livro introdutório sobre topologia geral, como por exemplo Lipschutz (1971) e Newman (1992). Também vale lembrar que muitas funções apresentadas ao longo do texto são representadas por meio de tabelas, como por exemplo as funções que representam imagens. Assim, em diversos algoritmos apresentados, tabelas que representam funções são construídas e a notação usada para atribuir um valor a função é a seguinte: $f(x) \leftarrow y$, onde $f$ é a função, $x$ um elemento do dominio da função e y é a imagem de $x$ em $f$ e este valor y é atribuído a tabela que representa a função $f$. 


\title{
Conceitos sobre imagens e operadores
} morfológicos

\begin{abstract}
Resumo do capítulo
Neste capítulo são apresentados conceitos, definições e notações relacionadas com imagens e operadores morfológicos que serão úteis ao longo do texto. Vale salientar, que neste texto, são assumidas definições usuais de conjuntos, relações, funções, grafos, reticulados e espaços topológicos que podem ser encontradas em um livro introdutório sobre topologia geral, como por exemplo Lipschutz (1971); Newman (1992).
\end{abstract}

\subsection{Definições básicas sobre imagens}

Como definido na Seção 1.1, neste trabalho trata-se uma imagem $f \in \mathcal{F}(\mathcal{D})$ como um mapeamento de uma grade retangular e finita $\mathcal{D} \subset \mathbb{Z} \times \mathbb{Z}$ em um conjunto discreto $\mathbb{K}=\{0, \ldots, K\}$ de níveis de cinza (isto é, $f: \mathcal{D} \rightarrow \mathbb{K}$ ), onde $K=2^{b}-1$ representa o nível de cinza máximo possível para uma imagem de $b>0$ bits de profundidade. Uma imagem binária, quando $b=1$, pode ter somente valores 0 ou 1 , e por este motivo é frequentemente representada como um subconjunto $\mathcal{X} \subseteq \mathcal{D}$ contendo os elementos de $\mathcal{D}$ cujos os valores são 1 , ou seja, $\mathcal{X}=\{x \in \mathcal{D}: f(x)=1\} \in \mathcal{P}(\mathcal{D})$. Por outro lado, uma imagem multibanda pode ser vista como um vetor de imagens $\left(f^{A}, f^{B}, \ldots, f^{Z}\right)$, onde $f^{A}, f^{B}, \ldots, f^{Z}$ fazem referências às bandas $A, B, \ldots, Z$ da imagem multibanda. Um elemento do domínio da imagem é chamado de pixel, ou seja, $p=\left(p_{x}, p_{y}\right) \in \mathcal{D}$ é um pixel cujo os valores $p_{x}$ e $p_{y}$ são os valores das coordenadas horizontal e vertical de $p$. Também, chama-se por $f(p)$ o valor do nível de cinza da imagem no pixel $p$. Além do mais, o pixel $p$ está conectado a outros pixels em seu entorno em $\mathcal{D}$ e esta noção de conectividade entre os pixels leva à definir uma relação de adjacência, denominada $\mathcal{A}$, sobre $\mathcal{D}$.

Definição 2.1 (Relação de adjacência). Uma relação de adjacência $\mathcal{A}$ sobre $\mathcal{D}$ é uma relação binária entre os pixels de $\mathcal{D}$, ou seja, $\mathcal{A} \subseteq \mathcal{D} \times \mathcal{D}$. Assim, se $(p, q) \in \mathcal{A}$ então é dito que $p$ é adjacente a $q$ ou alternativamente, $p$ é vizinho de $q$.

Em particular, neste trabalho são utilizadas somente relações de adjacências circulares e simétricas, ou seja, $(p, q) \in \mathcal{A}$ se, e somente se, $p, q \in \mathcal{D}, d(p, q) \leq \rho$, onde $d(p, q)=\sqrt{\left(p_{x}-q_{x}\right)^{2}+\left(p_{y}-q_{y}\right)^{2}}$ é a distância euclidiana entre $p$ e $q$, e $\rho$ é um fator de escala. Dessa forma, se $\rho=1$, temos a relação de adjacência, denotada por $\mathcal{A}_{4 c}$, que define a conhecida vizinhança-4 entre os pixels e analogamente se $\rho=\sqrt{2}$, temos o caso da vizinhança-8, denotada por $\mathcal{A}_{8 c}$, entre os pixels (ver 
Figura 2.1). Também, denotaremos o conjunto de pixels adjacentes a um dado pixel $p \in \mathcal{D}$ por $\mathcal{A}_{\mathcal{D}}(p)=\{q \in \mathcal{D}: p$ é adjacente a $q\}$ ou simplesmente por $\mathcal{A}(p)$ quando não existir ambiguidade.

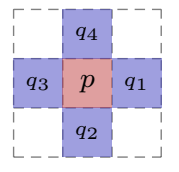

$\rho=1$

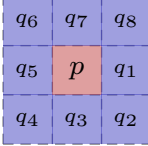

$\rho=\sqrt{2}$

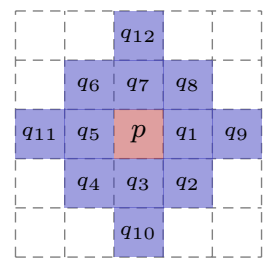

$\rho=2$

Figura 2.1: Exemplos de relações de adjacências para um dado pixel $p$.

Note que, uma relação binária $\mathcal{E}$ sobre um conjunto $\mathcal{V}$ dá origem a um grafo direcionado $\mathcal{G}=$ $(\mathcal{V}, \mathcal{E})$, onde o conjunto $\mathcal{V}$ é chamado de conjunto de vértices de $\mathcal{G}$ e $\mathcal{E}$ é chamado de conjunto de arestas de $\mathcal{G}$. Assim, a relação de adjacência $\mathcal{A}$ sobre $\mathcal{D}$, dá origem ao grafo da imagem $\mathcal{G}_{f}=(\mathcal{D}, \mathcal{A})$, onde os vértices são os próprios pixels e as arestas são definidas por $\mathcal{A}$. Com base nesta noção de conectividade entre os pixels, define-se:

Definição 2.2 (Caminho). Sejam $x$ e $y$ dois pixels de $\mathcal{X} \subseteq \mathcal{D}$. Diz-se que existe um caminho de $x$ até $y$ em $\mathcal{X}$, denotado por $\pi(x, y)$ se, e somente se, existir uma $n$-tupla de pixels distintos $\left(p_{1}, p_{2}, \ldots, p_{n}\right)$ tal que $p_{1}=x, p_{n}=y$ e $p_{i+1} \in \mathcal{A}_{\mathcal{X}}\left(p_{i}\right)$, para $1 \leq i \leq n-1$.

Definição 2.3 (Componente conexo). Um componente conexo $(\mathrm{CC}) \mathcal{C}$ é um subconjunto maximal de pixels de $\mathcal{X} \subseteq \mathcal{D}$, tal que para quaisquer dois pixels $p$ e $q \in \mathcal{C}$, existe um caminho de $p$ até $q$ em $\mathcal{C}$. Denota-se por $\mathcal{C} \mathcal{C}(\mathcal{X}, \mathcal{A})$ o conjunto dos CCs presentes em $\mathcal{X}$ definidos com a relação de adjacência $\mathcal{A}$ ou simplesmente por $\mathcal{C C}(\mathcal{X})$ quando $\mathcal{A}$ está bem definida. Na Figura 2.2 é apresentado um exemplo de extração de CCs para um dado conjunto $\mathcal{X}$ onde os CCs estão rotulados pelas cores: vermelho, verde e azul.

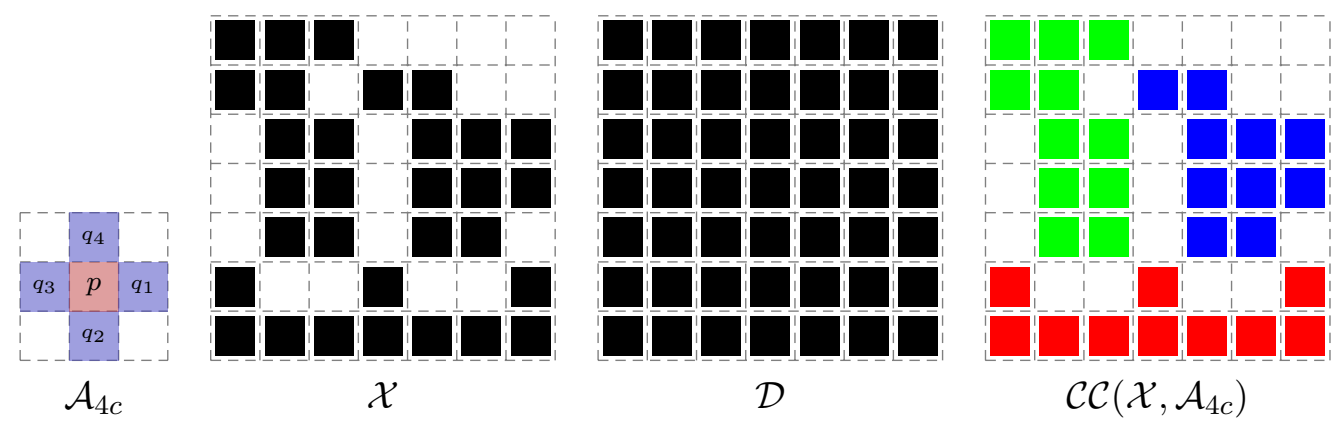

Figura 2.2: Exemplo de extração dos $C$ Cs do conjunto $\mathcal{X} \subseteq \mathcal{D}$ sobre a adjacência $\mathcal{A}_{4 c}$.

Definição 2.4 (Zona plana). Uma zona plana $\mathcal{C}$ de uma imagem $f \in \mathcal{F}(\mathcal{D})$ é um subconjunto maximal de pixels de $\mathcal{D}$, tal que para quaisquer dois pixels $p$ e $q \in \mathcal{C}$, existe uma $n$-tupla de pixels $\left(p_{1}=p, p_{2}, \ldots, p_{n}=q\right)$, satisfazendo $p_{i+1} \in \mathcal{A}\left(p_{i}\right)$ e $f\left(p_{i}\right)=f\left(p_{i+1}\right)$, para $1 \leq i \leq n-1$. Denota-se por $\mathcal{Z P}(f, \mathcal{A})$ o conjunto das zonas planas presentes em uma imagem $f$ definidas com a relação de adjacência $\mathcal{A}$, ou simplesmente por $\mathcal{Z P}(f)$ quando $\mathcal{A}$ está bem definida. Na Figura 2.3 é apresentado um exemplo de extração de zonas planas para uma dada imagem $f \in \mathcal{F}(\mathcal{D})$, onde as zonas planas estão rotuladas pelas cores: vermelha, verde, azul, amarela, laranja, ciano, violeta e cinza. 


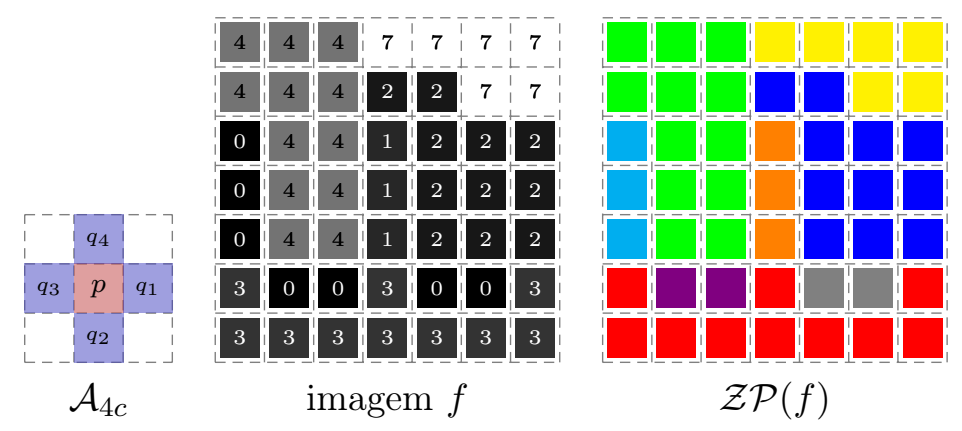

Figura 2.3: Exemplo de extração de zonas planas de uma imagem $f$ sob a adjacência $\mathcal{A}_{4 c}$.

Definição 2.5 (Extrema regional). Uma zona plana $\mathcal{C}$ é uma máxima (respectivamente, mínima) regional de uma imagem $f \in \mathcal{F}(\mathcal{D})$, se para todo pixel $p \in C$, satisfaz $f(p) \geq f\left(p_{i}\right)$ (respectivamente, $\left.f(p) \leq f\left(p_{i}\right)\right)$ com $p_{i} \in \mathcal{A}(p)$. Uma zona plana é uma extrema regional se ela é uma mínima ou máxima regional. Note que, as máximas (respectivamente, mínimas) regionais apresentadas na Figura 2.3 estão rotuladas nas cores: vermelha e amarela (respectivamente, ciano, violeta, cinza e laranja).

Definição 2.6 (Partição). Uma partição do conjunto $\mathcal{D}$, denotada por $\mathbb{P}$, é um conjunto não-vazio com $n$ regiões distintas $R_{i} \subseteq \mathcal{D}$, com $1 \leq i \leq n$, onde a união destas regiões é igual a $\mathcal{D}$, isto é:

1. A partição $\mathbb{P}$ é diferente de vazio, ou seja, $\mathbb{P} \neq \emptyset$;

2. Os elementos de $\mathbb{P}$ são disjuntos, isto é, se $R_{i}, R_{j} \in \mathbb{P}$ então $R_{i} \cap R_{j}=\emptyset$;

3. A união dos elementos de $\mathbb{P}$ é igual a $\mathcal{D}$, ou seja, $\cup\left\{R_{i}: R_{i} \in \mathbb{P}\right\}=\mathcal{D}$.

Denota-se por $\mathbb{P}_{f}$ a partição de $\mathcal{D}$ formada com as zonas planas de uma imagem $f \in \mathcal{F}(\mathcal{D})$, isto é, $\mathbb{P}_{f}=\{R: R \in \mathcal{Z} \mathcal{P}(f)\}$. Também, é denotada por $\mathbb{P}_{f}(p)$ a região de $\mathbb{P}_{f}$ contendo o pixel $p \in \mathcal{D}$.

Algumas vezes neste trabalho é exemplificado o uso de aplicações de operadores por meio de imagens simples cujo domínio é composto de um retângulo de altura 1. Essas imagens, podem ser representadas por gráficos de barras conhecidos por perfis de intensidades. Na Figura 2.4 é apresentado um exemplo de perfil de intensidade para uma dada imagem.

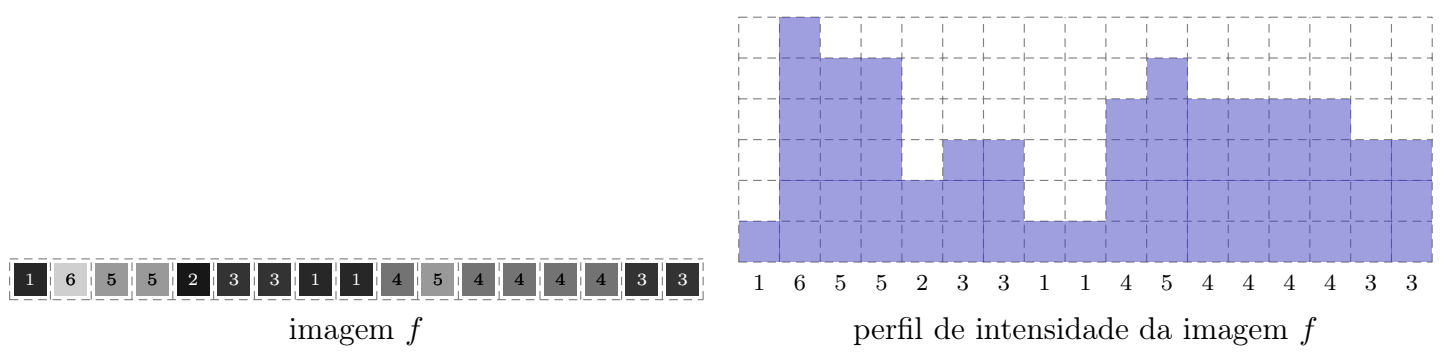

Figura 2.4: Exemplo de perfil de intensidade para uma dada imagem.

\subsection{Operadores morfológicos}

Como definido na Seção 1.1, em MM, operadores são vistos como mapeamentos entre reticulados completos (Heijmans, 1994; Najman e Talbot, 2013; Serra, 1982). Neste trabalho utiliza-se, basicamente, o reticulado dos subconjuntos $(\mathcal{P}(\mathcal{D}), \subseteq, \cap, \cup)$ e o reticulado das imagens $(\mathcal{F}(\mathcal{D}), \leq, \wedge, \vee)$. 
Dessa forma, um operador para subconjuntos (ou com um abuso de notação, para imagens binárias) é um mapeamento de $\mathcal{P}(\mathcal{D})$ em $\mathcal{P}(\mathcal{D})$ e analogamente, um operador para imagens (em níveis de cinza) é um mapeamento de $\mathcal{F}(\mathcal{D})$ em $\mathcal{F}(\mathcal{D})$. Eles podem ser caracterizados através de suas propriedades, as quais são fundamentais para prever os aspectos do seu comportamento. A seguir, são apresentadas brevemente algumas das propriedades elementares que podem ser associadas aos operadores (Banon e Barrera, 1994; Dougherty e Lotufo, 2003; Heijmans, 1994; Najman e Talbot, 2013; Serra, 1982; Soille, 2003).

- Crescente: Um operador de imagens $\psi$ (respectivamente, operador de subconjuntos $\Psi$ ) é crescente se preserva a relação de ordem que pode estar presente entre duas imagens (respectivamente, subconjuntos), ou seja, $\forall f, g \in \mathcal{F}(\mathcal{D})$ se $f \leq g$ então $\psi(f) \leq \psi(g)$ (respectivamente, $\forall X, Y \in \mathcal{P}(\mathcal{D})$ se $X \subseteq Y$ então $\Psi(X) \subseteq \Psi(Y))$.

- Idempotência: Um operador de imagens $\psi$ (respectivamente, operador de subconjuntos $\Psi$ ) é dito idempotente se, ao aplicá-lo mais de uma vez na mesma imagem (respectivamente, subconjunto) o resultado permanece inalterado, ou seja, $\forall f \in \mathcal{F}(\mathcal{D}), \psi(\psi(f))=\psi(f)$ (respectivamente, $X \in \mathcal{P}(\mathcal{D}), \Psi(\Psi(X))=\Psi(X))$.

- Extensividade: Um operador de imagens $\psi$ (respectivamente, operador de subconjuntos $\Psi$ ) é extensivo se, a entrada do operador é sempre comparável com o resultado da aplicação do operador, ou seja, $\forall f \in \mathcal{F}(\mathcal{D}), f \leq \psi(f)$ (respectivamente, $X \in \mathcal{P}(\mathcal{D}), X \subseteq \Psi(X)$ ).

- Anti-extensividade: Um operador de imagens $\psi$ (respectivamente, operador de subconjuntos $\Psi)$ é anti-extensivo se, o resultado da aplicação do operador é sempre comparável com a entrada do operador, isto é, $\forall f \in \mathcal{F}(\mathcal{D}), \psi(f) \leq f$ (respectivamente, $X \in \mathcal{P}(\mathcal{D}), \Psi(X) \subseteq X$ ).

- Dualidade: Dois operadores de imagens $\psi$ e $\phi$ (respectivamente, operadores de subconjuntos $\Psi$ e $\Phi)$ são duais com respeito a complementação se, ao aplicar $\psi$ (respectivamente, $\Psi$ ) em uma imagem $f \in \mathcal{F}(\mathcal{D})$ (respectivamente, subconjunto $X \in \mathcal{P}(\mathcal{D})$ ) é equivalente a aplicar $\phi$ (respectivamente, $\Phi$ ) no complemento da imagem ${ }^{1} f$ (respectivamente, $X$ ) e tomar o complemento do resultado do operador, ou seja, $f \in \mathcal{F}(\mathcal{D}), \psi(f)=\left[\phi\left(f^{c}\right)\right]^{c}$ (respectivamente, $\left.\forall X, Y \in \mathcal{P}(\mathcal{D}), \Psi(X)=\left[\Phi\left(X^{c}\right)\right]^{c}\right)$.

- Auto-dualidade: Um operador de imagens $\psi$ (respectivamente, operador de subconjuntos $\Psi$ ) é auto-dual se é dual de si mesmo, isto é, $\forall f \in \mathcal{F}(\mathcal{D}), \psi(f)=\left[\psi\left(f^{c}\right)\right]^{c}$ (respectivamente, $\left.\forall X \in \mathcal{P}(\mathcal{D}), \Psi(X)=\left[\Psi\left(X^{c}\right)\right]^{c}\right)$.

- Complementar: Dois operadores de imagens $\psi$ e $\phi$ (respectivamente, operadores de subconjuntos $\Psi$ e $\Phi)$ são complementares com respeito a complementação se ao aplicar $\psi$ (respectivamente, $\Psi$ ) em uma imagem $f \in \mathcal{F}(\mathcal{D})$ (respectivamente, subconjunto $X \in \mathcal{P}(\mathcal{D})$ ) é equivalente a aplicar $\phi$ (respectivamente, $\Phi)$ no complemento da imagem $f$ (respectivamente, $X$ ), ou seja, $f \in \mathcal{F}(\mathcal{D}), \psi(f)=\phi\left(f^{c}\right)$ (respectivamente, $\forall X, Y \in \mathcal{P}(\mathcal{D}), \Psi(X)=\Phi\left(X^{c}\right)$ ).

- Auto-complementar: Um operador de imagens $\psi$ (respectivamente, operador de subconjuntos $\Psi)$ é auto-complementar se é complementar de si mesmo, isto é, $\forall f \in \mathcal{F}(\mathcal{D}), \psi(f)=\psi\left(f^{c}\right)$ (respectivamente, $\forall X \in \mathcal{P}(\mathcal{D}), \Psi(X)=\Psi\left(X^{c}\right)$ ).

\footnotetext{
${ }^{1} \mathrm{O}$ complemento de uma imagem $f \in \mathcal{F}(\mathcal{D})$, denotado por $f^{c}$, é definido para $\forall x \in \mathcal{D}, f^{c}(x)=K-f(x)$.
} 
Em MM, muitos operadores são definidos com base em um conjunto $\mathcal{B} \subseteq \mathbb{Z}^{2}$ chamado elemento estruturante (EE). Esses operadores transformam o valor de um pixel $p \in \mathcal{D}$ de uma imagem $f \in \mathcal{F}(\mathcal{D})$ em um valor obtido utilizando informações dos pixels adjacentes a $p$. A relação de adjacência envolvida é definida por meio do conjunto $\mathcal{B}$ da seguinte forma: $p$ é adjacente a $q$ segundo $\mathcal{B}$ se, e somente se, $q \in(\mathcal{B}+p)=\{b+p: b \in \mathcal{B}\}$ e equivalentemente $(q-p) \in \mathcal{B}$ ou ainda $(p-q) \in \breve{\mathcal{B}}=\{-b: b \in \mathcal{B}\}$. Assim, denota-se por $\mathcal{A}_{\mathcal{B}}$ esta relação de adjacência definida pelo EE $\mathcal{B}$.

Dois operadores fundamentais baseados em EE são: erosão e dilatação. Aliás, uma grande quantidade de operadores morfológicos baseá-se numa combinação selecionada de erosão e dilatação. A erosão e a dilatação, denotadas por $\varepsilon_{\mathcal{B}}$ e $\delta_{\mathcal{B}}$, para conjuntos são definidas pela subtração e soma de Minkowski (Banon e Barrera, 1994; Dougherty e Lotufo, 2003) para todo $\mathcal{X} \in \mathcal{P}(\mathcal{D})$ como

$$
\varepsilon_{\mathcal{B}}(\mathcal{X})=\mathcal{X} \odot \mathcal{B}=\bigcap_{b \in \mathcal{B}}\{\mathcal{X}-b\} \text { e } \delta_{\mathcal{B}}(\mathcal{X})=\mathcal{X} \oplus \mathcal{B}=\bigcup_{b \in \mathcal{B}}\{\mathcal{X}+b\}
$$

e redefinida para toda imagem $f \in \mathcal{F}(\mathcal{D})$ como

$$
\forall p \in \mathcal{D},\left[\varepsilon_{\mathcal{B}}(f)\right](p)=\bigwedge_{b \in \mathcal{B}}\{f(p-b)\} \text { e } \forall p \in \mathcal{D},\left[\delta_{\mathcal{B}}(f)\right](p)=\bigvee_{b \in \mathcal{B}}\{f(p+b)\}
$$

Vale salientar que alguns autores, como por exemplo Soille (2003) e Serra (1982), definem a erosão e a dilatação para cada pixel $p \in \mathcal{D}$ como o ínfimo e o supremo dos pixels adjacentes a $p$ segundo $\mathcal{B}$. Neste caso, o $\mathrm{EE} \mathcal{B}$ não é refletido na definição do operador de erosão. Mas é claro, se o EE $\mathcal{B}$ é simétrico em relação a origem, então $\mathcal{B}=\breve{\mathcal{B}}$ e assim as definições ficam exatamente iguais. Na Figura 2.5 é apresentado um exemplo das aplicações de erosão e dilatação com um EE simétrico. Observe que uma das características da erosão (respectivamente, dilatação) é aumentar as regiões escuras (respectivamente, claras) e diminuir as regiões claras (respectivamente, escuras) presentes na imagem, de acordo com o formato geométrico do EE (Dougherty e Lotufo, 2003; Soille, 2003).

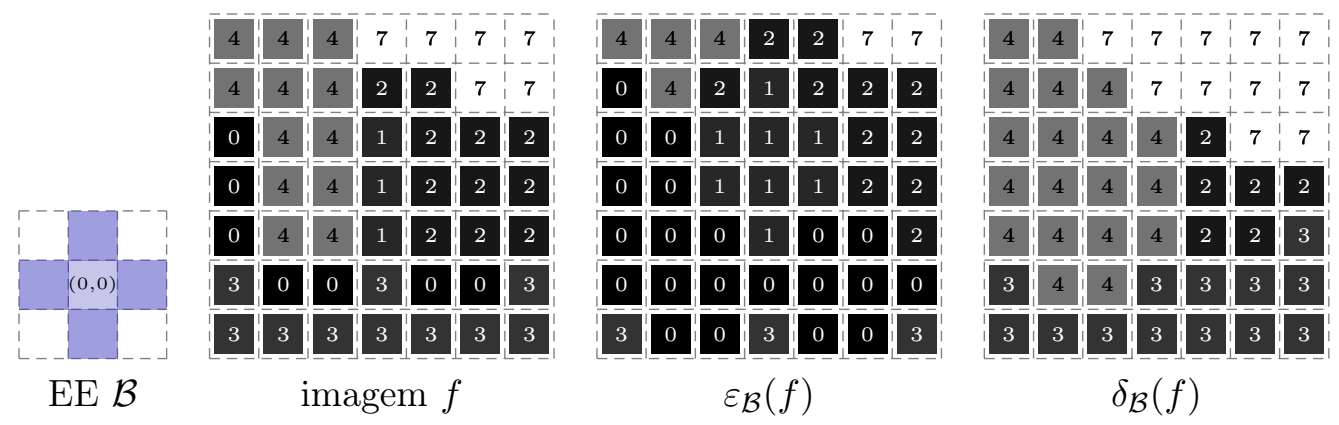

Figura 2.5: Exemplo da aplicação de erosão e dilatação com EE formato de cruz em uma dada imagem.

A erosão $\varepsilon_{\mathcal{B}}$ e a dilatação $\delta_{\mathcal{B}}$ são operadores duais, crescentes e se $\mathcal{B}$ é simétrico em relação a origem então a erosão é anti-extensiva e a dilatação é extensiva. A composição sequencial de erosão e dilatação levam às definições de outros dois operadores básicos chamados de abertura e fechamento, denotados por $\gamma_{\mathcal{B}}$ e $\varphi_{\mathcal{B}}$, e definidos para todo conjunto $\mathcal{X} \in \mathcal{P}(\mathcal{D})$ como

$$
\gamma_{\mathcal{B}}(\mathcal{X})=\varepsilon_{\mathcal{B}}\left(\delta_{\mathcal{B}}(\mathcal{X})\right) \text { e } \varphi_{\mathcal{B}}(\mathcal{X})=\delta_{\mathcal{B}}\left(\varepsilon_{\mathcal{B}}(\mathcal{X})\right)
$$


e analogamente, para toda imagem $f \in \mathcal{F}(\mathcal{D})$ como

$$
\gamma_{\mathcal{B}}(f)=\varepsilon_{\mathcal{B}}\left(\delta_{\mathcal{B}}(f)\right) \text { e } \varphi_{\mathcal{B}}(f)=\delta_{\mathcal{B}}\left(\varepsilon_{\mathcal{B}}(f)\right)
$$

Como o par de operadores erosão e dilatação, o par abertura e fechamento também são duais, crescentes e, se $\mathcal{B}$ é simétrico em relação a origem, então a abertura é anti-extensiva e o fechamento é extensivo. Contudo, eles têm uma propriedade a mais, a idempotência. Vale salientar que, operadores crescentes e idempotentes são conhecidos como filtros morfológicos (Serra e Vincent, 1992). Na Figura 2.6 é apresentado um exemplo das aplicações de abertura e fechamento com um EE simétrico com formato geométrico de cruz.

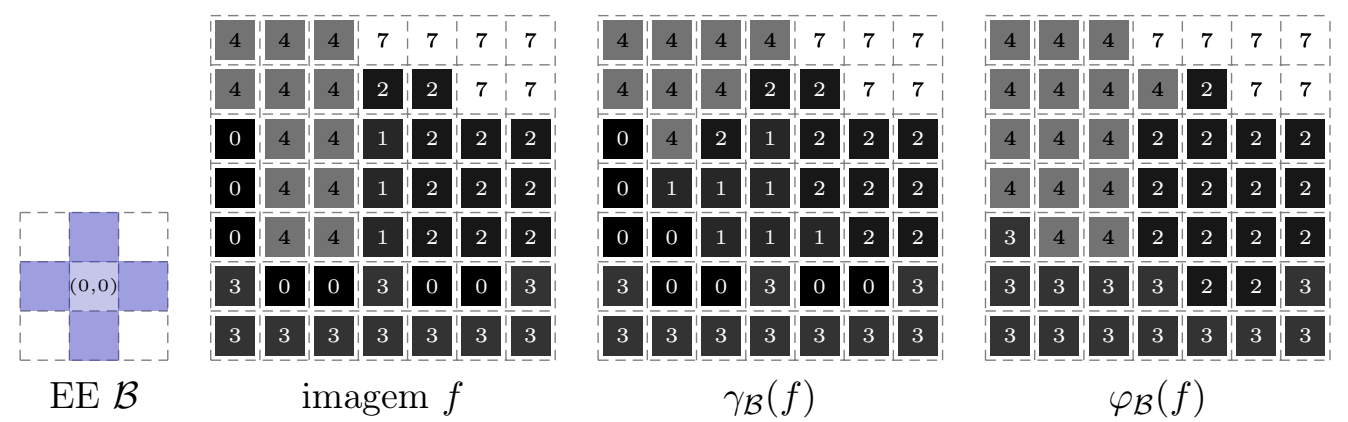

Figura 2.6: Exemplo da aplicação de abertura e fechamento com EE formato de cruz em uma dada imagem.

Os operadores morfológicos discutidos até este momento envolvem combinações de um conjunto ou imagem com um EE. Por outro lado, alguns operadores evolvem combinações de um conjunto ou imagem, denominado marcador, com um EE e um outro conjunto ou imagem chamado máscara. Por exemplo, a dilatação condicional para conjuntos, $\delta_{\mathcal{B}}^{1}(\mathcal{X}, \mathcal{M})$ é a dilatação do conjunto (marcador) $\mathcal{M} \subseteq \mathcal{X}$ pelo EE $\mathcal{B}$ condicionado por um outro conjunto (máscara) $\mathcal{X} \in \mathcal{P}(\mathcal{D})$, ou seja,

$$
\delta_{\mathcal{B}}^{1}(\mathcal{X}, \mathcal{M})=\delta_{\mathcal{B}}(\mathcal{M}) \cap \mathcal{X}
$$

A dilatação condicional pode ser utilizada para reconstruir CCs do conjunto $\mathcal{X}$ marcados pelo conjunto $\mathcal{M}$. A reconstrução é obtida após $n$ aplicações sucessivas da dilatação condicional $\delta_{\mathcal{B}}^{n}(\mathcal{X}, \mathcal{M})$ até atingir a idempotência, isto é, $\delta_{\mathcal{B}}^{n}(\mathcal{X}, \mathcal{M})=\delta_{\mathcal{B}}\left(\delta_{\mathcal{B}}^{n}(\mathcal{X}, \mathcal{M})\right) \cap \mathcal{X}$. Este procedimento, ilustrado pela Figura 2.7, dá origem ao operador reconstrução por dilatação $\delta_{\mathcal{B}}^{\infty}(\mathcal{X}, \mathcal{M})$ de um conjunto $\mathcal{X} \in \mathcal{P}(\mathcal{D})$ por um EE $\mathcal{B}$ utilizando o conjunto marcador $\mathcal{M} \subseteq \mathcal{X}$ e definida por

$$
\delta_{\mathcal{B}}^{\infty}(\mathcal{X}, \mathcal{M})=\delta_{\mathcal{B}}^{n}(\mathcal{X}, \mathcal{M})
$$

em que $\delta_{\mathcal{B}}^{n}(\mathcal{X}, \mathcal{M})$ é a dilatação condicional com $n>0$ iterações satisfazendo $\mathcal{M} \subseteq \mathcal{X}$ e $\delta_{\mathcal{B}}^{n}(\mathcal{X}, \mathcal{M})=$ $\delta_{\mathcal{B}}\left(\delta_{\mathcal{B}}^{n}(\mathcal{X}, \mathcal{M})\right) \cap \mathcal{M}$ e definida por

$$
\delta_{\mathcal{B}}^{n}(\mathcal{X}, \mathcal{M})= \begin{cases}\delta_{\mathcal{B}}(\mathcal{X}) \cap \mathcal{X}, & \text { se } \mathrm{n}=1 \\ \delta_{\mathcal{B}}\left(\delta_{\mathcal{B}}^{n-1}(\mathcal{X}, \mathcal{M})\right) \cap \mathcal{X}, & \text { caso contrário. }\end{cases}
$$

Analogamente, como ilustrado na Figura 2.8, a reconstrução por erosão $\varepsilon_{\mathcal{B}}^{\infty}(\mathcal{X}, \mathcal{M})$ de um conjunto $\mathcal{X} \in \mathcal{P}(\mathcal{D})$ por um EE $\mathcal{B}$ utilizando o conjunto marcador $\mathcal{M} \supseteq \mathcal{X}$ é definida por $\varepsilon_{\mathcal{B}}^{\infty}(\mathcal{X}, \mathcal{M})=\varepsilon_{\mathcal{B}}^{n}(\mathcal{X}, \mathcal{M})$ onde $\varepsilon_{\mathcal{B}}^{n}(\mathcal{X}, \mathcal{M})$ é a erosão condicional com $n>0$ iterações satisfazendo 


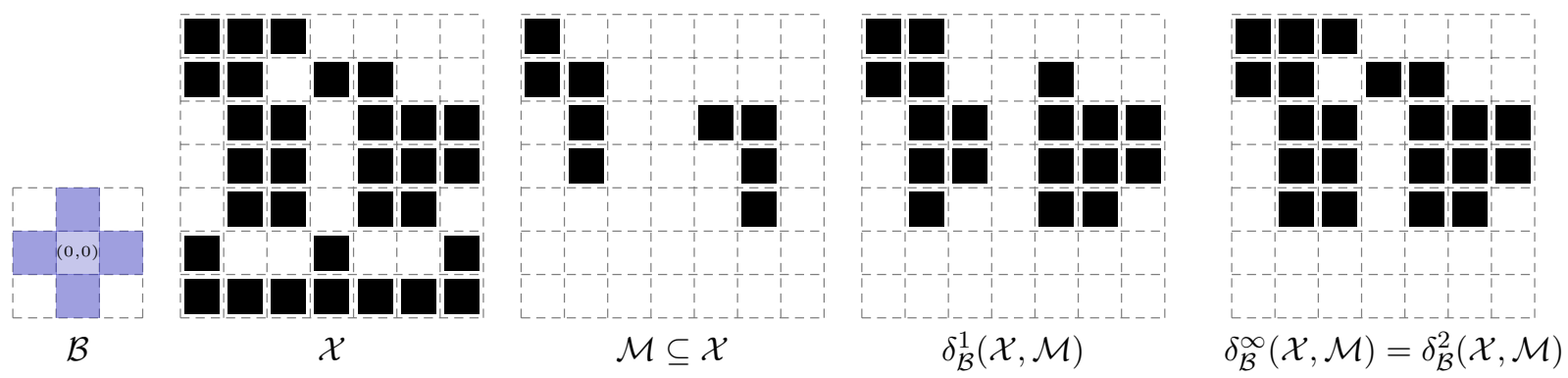

Figura 2.7: Exemplo de reconstrução por dilatação para um conjunto $\mathcal{X}$ e um conjunto marcador $\mathcal{M}$.

$\mathcal{X} \subseteq \mathcal{M}$ e $\varepsilon_{\mathcal{B}}^{n}(\mathcal{X}, \mathcal{M})=\varepsilon_{\mathcal{B}}\left(\varepsilon_{\mathcal{B}}^{n}(\mathcal{X}, \mathcal{M})\right) \cup \mathcal{X}$ e definida por

$$
\varepsilon_{\mathcal{B}}^{n}(\mathcal{X}, \mathcal{M})= \begin{cases}\varepsilon_{\mathcal{B}}(\mathcal{M}) \cup \mathcal{X}, & \text { se } \mathrm{n}=1, \\ \varepsilon_{\mathcal{B}}\left(\varepsilon_{\mathcal{B}}^{n-1}(\mathcal{X}, \mathcal{M})\right) \cup \mathcal{X}, & \text { caso contrário, }\end{cases}
$$

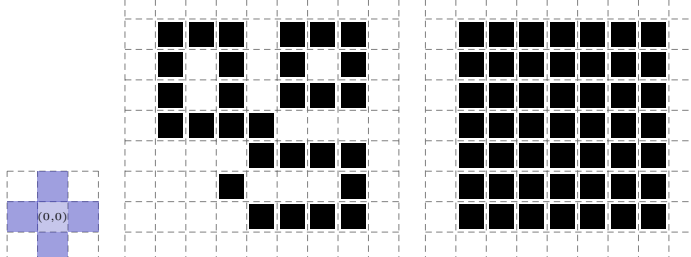

$\mathcal{M}$

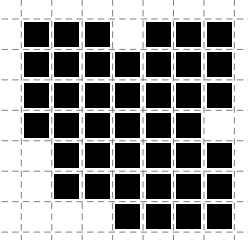

$\varepsilon_{\mathcal{B}}^{1}(\mathcal{X}, \mathcal{M})$

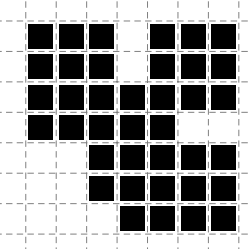

$\varepsilon_{\mathcal{B}}^{2}(\mathcal{X}, \mathcal{M})$

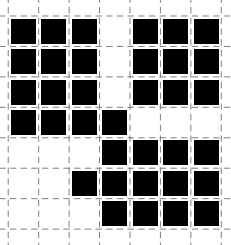

$\varepsilon_{\mathcal{B}}^{\infty}(\mathcal{X}, \mathcal{M})=\varepsilon_{\mathcal{B}}^{3}(\mathcal{X}, \mathcal{M})$

Figura 2.8: Exemplo de reconstrução por erosão para um conjunto $\mathcal{X}$ e um conjunto marcador $\mathcal{M}$.

Estes operadores podem ser facilmente estendidos para imagens simplesmente utilizando os elementos: relação de ordem $(\leq)$, ínfimo $(\wedge)$ e supremo $(\vee)$ do reticulado das imagens, ao invés dos elementos: relação de ordem $(\subseteq)$, ínfimo $(\cap)$ e supremo $(\cup)$ do reticulado dos subconjuntos. Assim, a dilatação por reconstrução, ilustrada na Figura 2.9, para uma imagem $f \in \mathcal{F}(\mathcal{D})$ por um EE $\mathcal{B}$ utilizando uma imagem marcadora $g \in \mathcal{F}(\mathcal{D})$ com $g \leq f$ é definida da seguinte forma:

$$
\delta_{\mathcal{B}}^{\infty}(f, g)=\delta_{\mathcal{B}}^{n}(f, g)
$$

em que $\delta_{\mathcal{B}}^{n}(f, g)=\delta_{\mathcal{B}}\left(\delta_{\mathcal{B}}^{n}(f, g)\right) \wedge f$.

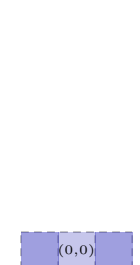

EE $\mathcal{B}$

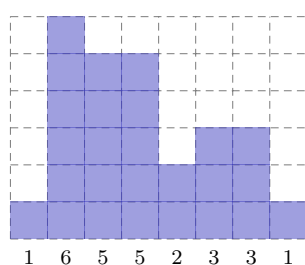

máscara $f$

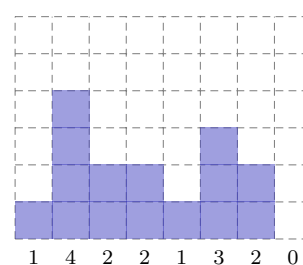

marcador $g$

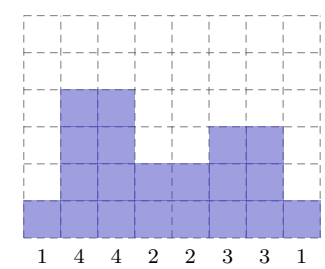

$\delta_{\mathcal{B}}^{1}(f, g)$

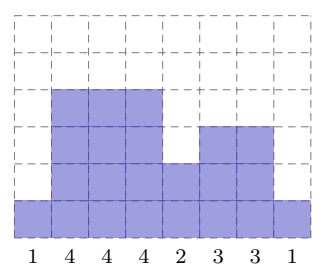

$\delta_{\mathcal{B}}^{\infty}(f, g)=\delta_{\mathcal{B}}^{2}(f, g)$

Figura 2.9: Exemplo de reconstrução por dilatação para um imagem $f$ e uma imagem marcadora $g$.

Analogamente, a reconstrução por erosão, ilustrada na Figura 2.10, para uma imagem $f \in \mathcal{F}(\mathcal{D})$ por um EE $\mathcal{B}$ utilizando uma imagem marcadora $g \in \mathcal{F}(\mathcal{D})$ com $f \leq g$ é definida da seguinte forma:

$$
\varepsilon_{\mathcal{B}}^{\infty}(f, g)=\varepsilon_{\mathcal{B}}^{n}(f, g)
$$

em que $\varepsilon_{\mathcal{B}}^{n}(f, g)=\varepsilon_{\mathcal{B}}\left(\varepsilon_{\mathcal{B}}^{n}(f, g)\right) \vee f$. 


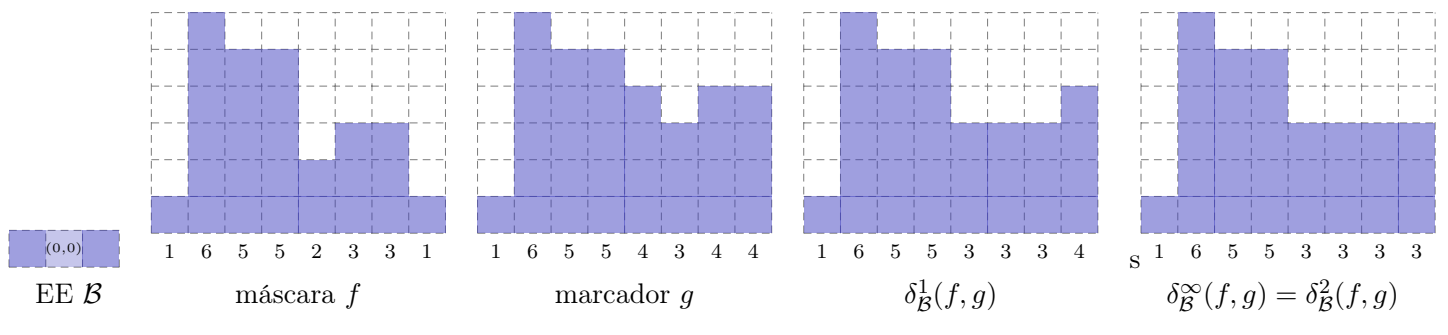

Figura 2.10: Exemplo de reconstrução por erosão para um imagem $f$ e uma imagem marcadora $g$.

A reconstrução por erosão e dilatação são duais, crescentes e idempotentes, ou seja, são filtros morfológicos, e se $\mathcal{B}$ é simétrico em relação a origem, então a abertura é anti-extensiva e o fechamento é extensivo. Aliás, uma grande quantidade de operadores morfológicos são definidos a partir da reconstrução por erosão e dilatação, como por exemplo: abertura e fechamento por reconstrução, $h$ bacias, $h$-domos, abertura e fechamento top-hat por reconstrução, entre outros (Dougherty e Lotufo, 2003; Soille, 2003). Não obstante, erosão e a dilatação por reconstrução fazem parte de uma classe de operadores que preservam contornos, os operadores conexos.

Outros operadores morfológicos utilizados para reconstruir regiões de uma imagem são os filtros por atributos (Breen e Jones, 1996) que também são operadores conexos. Dentre os filtros por atributos (Breen e Jones, 1996; Caselles e Monasse, 2002; Salembier e Wilkinson, 2009; Salembier et al., 1998; Vincent, 1994), a abertura (respectivamente, fechamento) por atributo desempenha um papel similar à reconstrução por dilatação (respectivamente, erosão), sendo a principal diferença a forma com que é realizada a reconstrução, a qual é determinada por um critério sobre o valor de um atributo crescente, enquanto que a reconstrução por dilatação (respectivamente, erosão), é determinada por uma imagem marcadora. O atributo crescente pode ser representado por uma função $\kappa: \mathcal{P}(\mathcal{D}) \rightarrow \mathbb{R}$ tal que se $A, B \in \mathcal{P}(\mathcal{D})$ com $A \subseteq B$ então $\kappa(A) \leq \kappa(B)$. Exemplos de atributos crescentes incluem: área, volume, altitude, entre outros. Dessa forma, a abertura por atributo para um conjunto $\mathcal{X} \in \mathcal{P}(\mathcal{D})$ é um conjunto $\gamma_{i}^{\kappa}(\mathcal{X})$ contendo os CCs de $\mathcal{X}$ definidos por uma relação de adjacência $\mathcal{A}$ que satisfazem o critério definido sobre o valor do atributo $\kappa$, isto é,

$$
\gamma_{i}^{\kappa}(\mathcal{X})=\left\{C \in \mathcal{C C}_{\mathcal{A}}(\mathcal{X}): \kappa(C)>i\right\}
$$

e por dualidade pode-se definir o fechamento por atributos, como $\phi_{i}^{\kappa}(\mathcal{X})=\left[\gamma_{i}^{\kappa}\left(\mathcal{X}^{c}\right)\right]^{c}$. Na Figura 2.11 são apresentados alguns exemplo de aplicações de aberturas e fechamentos por atributos.

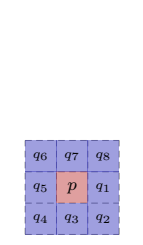

Adjacência

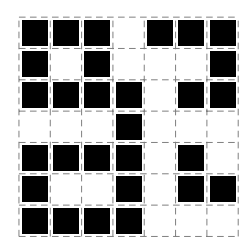

conjunto $\mathcal{X}$

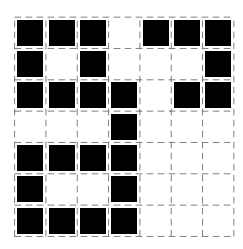

abertura por área $\gamma_{3}^{\kappa}(\mathcal{X})$

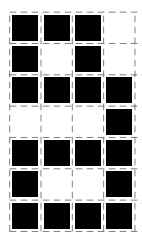

abertura por altura $\gamma_{3}^{\kappa}(\mathcal{X})$

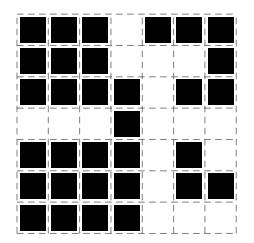

fechamento por área $\phi_{2}^{\kappa}(\mathcal{X})$ fechamento por altura $\phi_{1}^{\kappa}(\mathcal{X})$

Figura 2.11: Exemplos de aberturas e fechamentos por atributos.

O par abertura e fechamento por atributo são duais, crescentes e idempotentes, ou seja, são filtros morfológicos. Como é conhecido, qualquer operador binário crescente, pode ser estendido para imagens por meio da decomposição por limiarização (Maragos e Ziff, 1990; Wendt et al., 1986) que é amplamente discutida no Capítulo 4. Pela decomposição por limiarização, a abertura e o fechamento 
por atributos são definidos para imagens da seguinte forma:

$$
\forall p \in \mathcal{D},\left[\gamma_{i}^{\kappa}(f)\right](p)=\max \left\{\lambda \in \mathbb{K}: p \in \gamma_{i}^{\kappa}\left(T_{\lambda}(f)\right), i \in \mathbb{R}\right\}
$$

onde $T_{\lambda}(f)$ é o tradicional operador limiarização da imagem $f$ pelo limiar $\lambda$, isto é, $T_{\lambda}(f)=$ $\{p \in \mathcal{D}: f(p) \geq \lambda\}$. Analogamente, o fechamento por atributo pode ser definido por dualidade $\phi_{i}^{\kappa}(f)=\left[\gamma_{i}^{\kappa}\left(f^{c}\right)\right]^{c}$. Na Figura 2.12 são apresentados alguns exemplos de aplicações de aberturas e fechamentos por atributos em imagens.
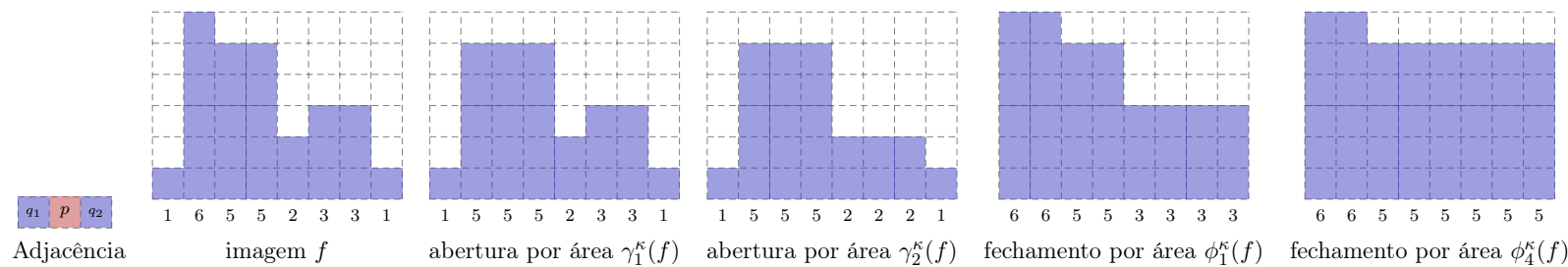

Figura 2.12: Exemplos de aberturas e fechamentos por atributos. 


\section{Operadores conexos e suas especializações}

\section{Resumo do capítulo}

Neste capitulo são apresentados conceitos, definições e notações sobre operadores conexos e suas especializações como os levelings e monotone planings. São mostradas também propriedades e relações que destacam a representação do espaço de escala com base em levelings proposto por Meyer e Maragos (1999).

\subsection{Conceitos básicos sobre operadores conexos e suas especializações}

Neste trabalho, tem-se interesse em uma subclasse específica de operadores morfológicos, os operadores conexos. Eles agem ampliando zonas planas adjacentes por meio de um processo de fusão e, por consequência, acabam não criando, na imagem de saída, novas estruturas (como contornos) que já não estejam presentes na imagem de entrada (Salembier e Serra, 1995; Serra e Salembier , 1993). Na Figura 3.1 são apresentados alguns exemplos de aplicações de operadores de imagens em geral, para uma simples comparação, no intuito de eliminar ruídos.
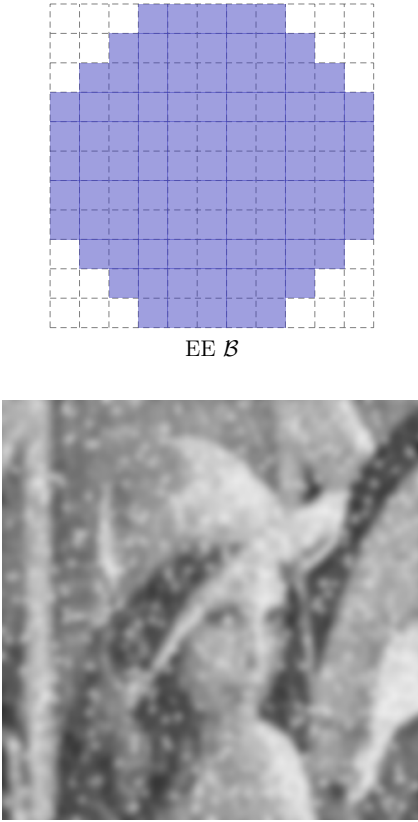

filtro gaussiano (núcleo definido por $\mathcal{B}$ )

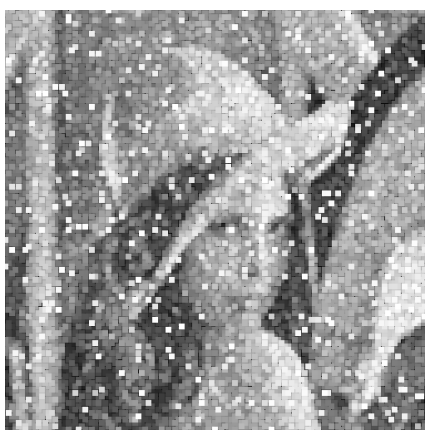

Imagem de entrada $f$ ruidosa

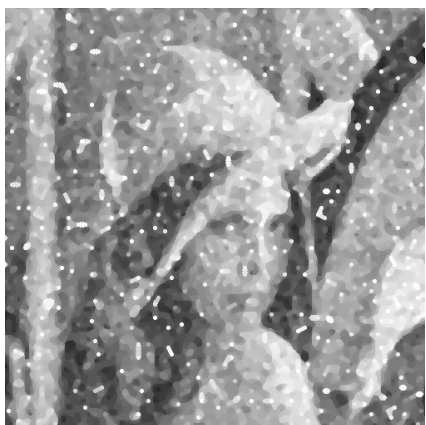

filtro da mediana (núcleo definido por $\mathcal{B}$ )

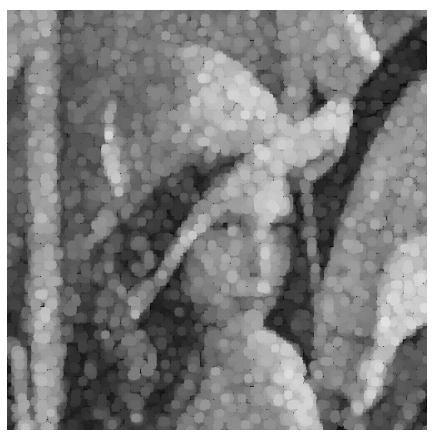

Abertura $\gamma_{\mathcal{B}}(f)$

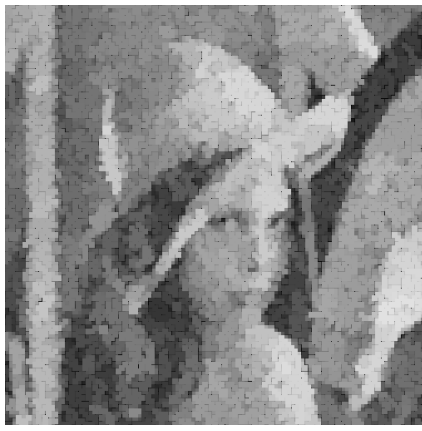

operador conexo

Figura 3.1: Exemplo de aplicações de operadores para fins de filtragem. 
Os operadores conexos, originalmente introduzidos por Serra e Salembier (1993), foram definidos com base na partição $\mathbb{P}_{f}$ de $\mathcal{D}$ formada pelas zonas planas de uma imagem $f \in \mathcal{F}(\mathcal{D})$.

Definição 3.1 (Operador conexo (Serra e Salembier, 1993)). Um operador $\psi: \mathcal{F}(\mathcal{D}) \rightarrow \mathcal{F}(\mathcal{D})$ é dito conexo, se para toda imagem $f \in \mathcal{F}(\mathcal{D})$, a seguinte relação é válida para todos os pixels $p \in \mathcal{D}$,

$$
\mathbb{P}_{f}(p) \subseteq \mathbb{P}_{\psi(f)}(p)
$$

Esta definição destaca o processamento baseado em regiões, uma vez que as regiões da partição da imagem de saída são criadas por fusões de regiões adjacentes da partição da imagem de entrada. Na Figura 3.2 são apresentados exemplos de partições de operadores conexos para uma dada imagem.

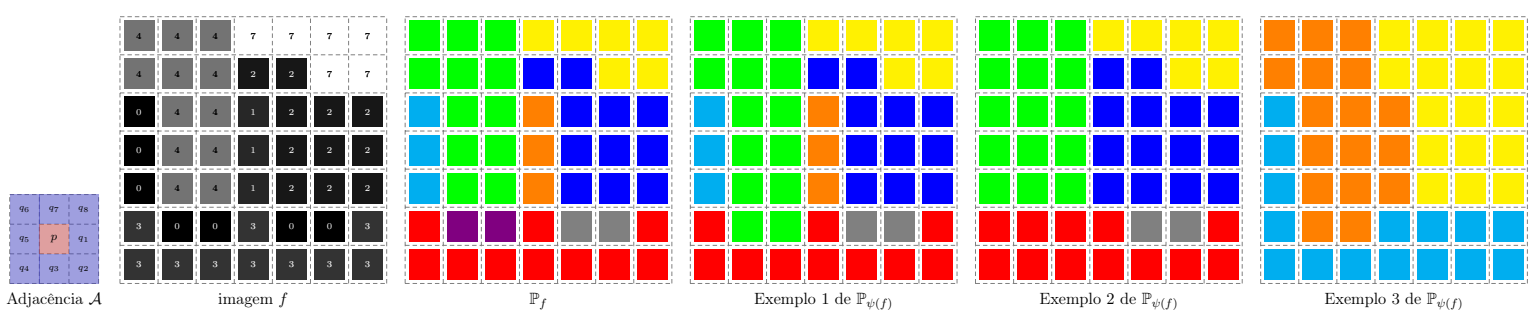

Figura 3.2: Exemplos de partições de operadores conexos para uma dada imagem.

Uma definição equivalente, introduzida por Meyer (1998b), destaca o processamento entre as fronteiras das regiões, em outras palavras, quando houver uma transição entre dois pixels adjacentes, digamos $(p, q) \in \mathcal{A}$, na imagem de saída (isto é, $[\psi(f)](p) \neq[\psi(f)](q)$ ). Essa transição também ocorre entre os mesmos pixels na imagem de entrada (isto é, $f(p) \neq f(q)$ ).

Definição 3.2 (Operador conexo (Meyer, 1998a,b)). Um operador $\psi: \mathcal{F}(\mathcal{D}) \rightarrow \mathcal{F}(\mathcal{D})$ é dito conexo, se para toda imagem $f \in \mathcal{F}(\mathcal{D})$, a seguinte relação é válida para todos os pares de pixels adjacentes, ou seja, $\forall(p, q) \in \mathcal{A}$, temos que:

$$
\text { se }[\psi(f)](p) \neq[\psi(f)](q) \text { então } f(p) \neq f(q) \text {, }
$$

ou equivalentemente (pelo significado lógico: $A \Rightarrow B$ é equivalente a $\neg B \Rightarrow \neg A$ ),

$$
\text { se } f(p)=f(q) \text { então }[\psi(f)](p)=[\psi(f)](q) \text {. }
$$

Os operadores conexos têm em comum a característica de preservar ou criar novas zonas planas por meio de fusões de zonas planas adjacentes. Neste sentido, muitas zonas planas podem se tornar extremas (mínimas ou máximas) regionais na imagem de saída (Salembier e Serra, 1995). Com base neste fato, Meyer (1998a,b) estudou duas subclasses de operadores conexos que não criam novas extremas regionais. A primeira denominada de levelings e a segunda de monotone planings.

Os operadores levelings são poderosos filtros para simplificação de imagens que preservam a ordem das transições, não criam novas estruturas (extremas regionais e contornos) e seus valores são delimitados por valores de uma vizinhança de pixels. 
Definição 3.3 (Operador leveling (Meyer, 1998a,b)). Um operador $\psi: \mathcal{F}(\mathcal{D}) \rightarrow \mathcal{F}(\mathcal{D})$ é dito leveling, se para toda imagem $f \in \mathcal{F}(\mathcal{D})$, a seguinte relação é válida para todos os pares de pixels adjacentes, ou seja, $\forall(p, q) \in \mathcal{A}$, temos que:

$$
\text { se }[\psi(f)](p)>[\psi(f)](q) \text { então } f(p) \geq[\psi(f)](p) \text { e }[\psi(f)](q) \geq f(q) \text {. }
$$

ou equivalentemente (pelo significado lógico: $A \Rightarrow B$ é equivalente a $\neg B \Rightarrow \neg A$ ),

$$
\text { se } f(p)<[\psi(f)](p) \leq[\psi(f)](q)<f(q) \text { então }[\psi(f)](p) \leq[\psi(f)](q) \text {. }
$$

Pela Definição 3.3, pode-se observar que quando existe uma transição $(p, q)$ em $\psi(f)$ então $f(p) \wedge f(q) \leq[\psi(f)](p) \wedge[\psi(f)](q)<[\psi(f)](p) \vee[\psi(f)](q) \leq f(p) \vee f(q)$, pois $f(p) \geq[\psi(f)](p)>$ $[\psi(f)](q) \geq f(q)$. Logo, $[\psi(f)](p)$ e $[\psi(f)](q)$ têm valores entre $f(p) \wedge f(q)$ e $f(p) \vee f(q)$, quando $[\psi(f)](p) \neq[\psi(f)](q)$. Basicamente, isso significa que qualquer transição entre duas regiões conexas de $\psi(f)$ é delimitada por uma grande variação entre os níveis destas regiões em $f$. Este fato, pode ser observado por meio de exemplos de operadores levelings apresentados na Figura 3.3.
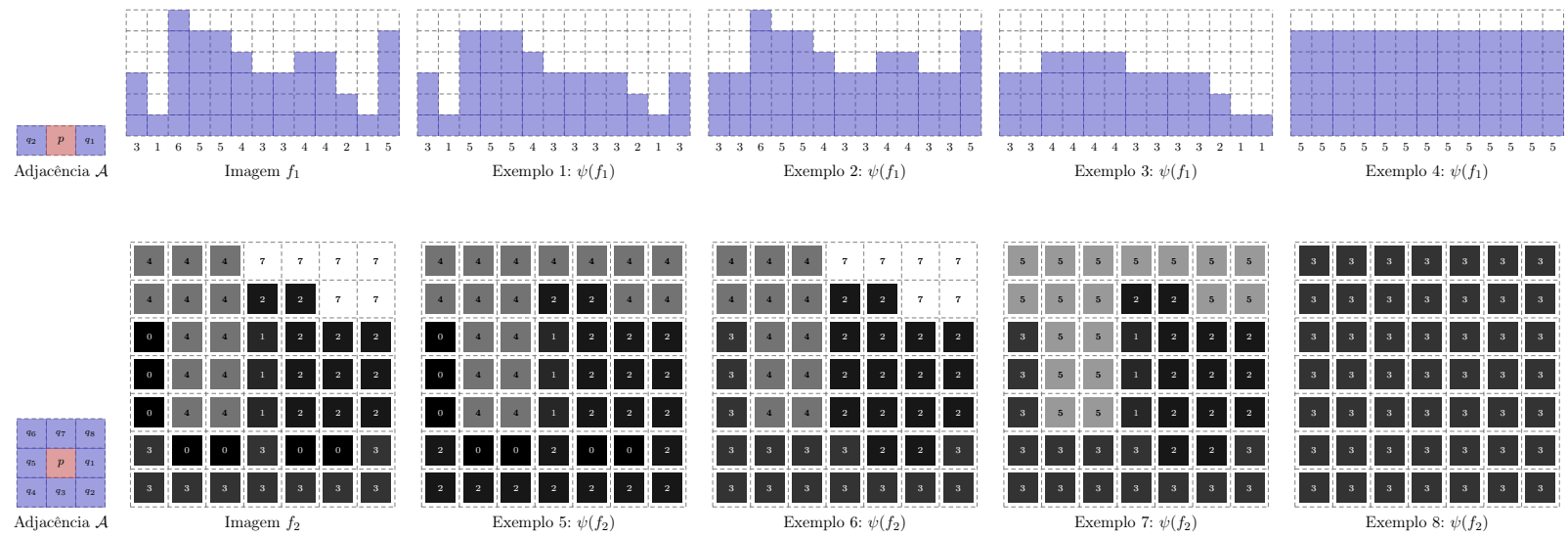

Figura 3.3: Exemplos de aplicações de operadores levelings em uma dada imagem.

Os levelings, uma subclasse de operadores conexos, estabelecem limites de valores de acordo com os valores de $f$, enquanto que os operadores conexos simplesmente ignoram tais limites. Uma outra subclasse dos operadores conexos, os monotone planing, também ignoram estes limites, mas preservam a ordem das transições de $f$ como os levelings. Na Figura 3.4 pode-se observar este fato por meio dos exemplos de aplicações de operadores monotone planing.

Definição 3.4 (Operador monotone planing (Meyer, 1998a,b)). Um operador $\psi: \mathcal{F}(\mathcal{D}) \rightarrow \mathcal{F}(\mathcal{D})$ é dito monotone planing, se para toda imagem $f \in \mathcal{F}(\mathcal{D})$, a seguinte relação é válida para todos os pares de pixels adjacentes, ou seja, $\forall(p, q) \in \mathcal{A}$, temos que:

$$
\text { se }[\psi(f)](p)>[\psi(f)](q) \text { então } f(p)>f(q) \text {. }
$$

ou equivalentemente (pelo significado lógico: $A \Rightarrow B \Longleftrightarrow \neg B \Rightarrow \neg A$ ),

$$
\text { se } f(p) \leq f(q) \text { então }[\psi(f)](p) \leq[\psi(f)](q) \text {. }
$$




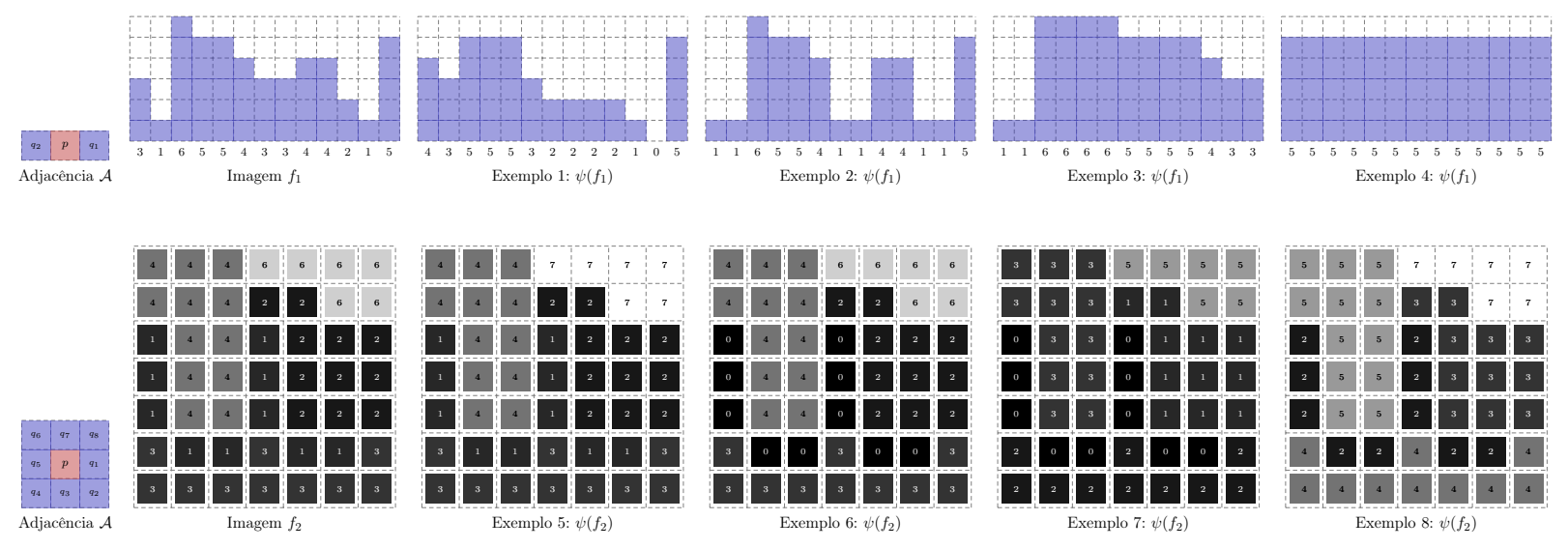

Figura 3.4: Exemplos de aplicações de operadores monotone planing em uma dada imagem.

Note que, os levelings podem ser vistos como operadores monotone planing que são limitados pelos valores de $f$.

\subsection{Operadores vistos como relações binárias}

Por meio das Definições 3.2, 3.3 e 3.4 das classes dos operadores conexos, levelings e monotone planing são possíveis construirmos relações binárias $\mathcal{E}_{\text {conexo }}, \mathcal{E}_{\text {leveling }}$ e $\mathcal{E}_{\text {monotone planing }}$ sobre $\mathcal{F}(\mathcal{D})$. Por exemplo, a relação binária $\mathcal{E}_{\text {conexo }} \subseteq \mathcal{F}(\mathcal{D}) \times \mathcal{F}(\mathcal{D})$ é construída da seguinte forma: $(g, f) \in$ $\mathcal{E}_{\text {conexo }}$ se, e somente se, existir um operador conexo $\psi$ tal que $g=\psi(f)$. Neste caso, dizemos que $g$ é conexo de $f$ se, e somente se, $(g, f) \in \mathcal{E}_{\text {conexo. Analogamente, define-se também as relações }}$ binárias $\mathcal{E}_{\text {leveling }} \subseteq \mathcal{F}(\mathcal{D}) \times \mathcal{F}(\mathcal{D})$ e $\mathcal{E}_{\text {monotone planing }} \subseteq \mathcal{F}(\mathcal{D}) \times \mathcal{F}(\mathcal{D})$. Estas relações binárias podem estar associadas a diversas propriedades, como por exemplo:

- reflexividade: Uma relação binária $\mathcal{E}_{\preccurlyeq}$ sobre $\mathcal{V}$ é dita reflexiva se, e somente se, para todo $x \in \mathcal{V}$ tem-se $(x, x) \in \mathcal{E}_{\preccurlyeq}$ (ou pela notação: $\left.x \preccurlyeq x\right)$;

- simetria: Uma relação binária $\mathcal{E}_{\preccurlyeq}$ sobre $\mathcal{V}$ é dita simétrica se, e somente se, para todo $x, y \in \mathcal{V}$ tal que se $(x, y) \in \mathcal{E}_{\preccurlyeq}$ então $(y, x) \in \mathcal{E}_{\preccurlyeq}$ (ou pela notação: se $x \preccurlyeq y$ então $y \preccurlyeq x$ );

- anti-simetria: Uma relação binária $\mathcal{E}_{\preccurlyeq}$ sobre $\mathcal{V}$ é dita anti-simétrica se, e somente se, para todo $x, y \in \mathcal{V}$ tal que se $(x, y),(y, x) \in \mathcal{E}_{\preccurlyeq}$ então $x=y$ (ou pela notação: se $x \preccurlyeq y$ e $y \preccurlyeq x$ então $x=y$ );

- transitividade: Uma relação binária $\mathcal{E}_{\preccurlyeq}$ sobre $\mathcal{V}$ é dita transitiva se, e somente se, para todo $x, y, z \in \mathcal{V}$ tal que se $(x, y),(y, z) \in \mathcal{E}_{\preccurlyeq}$ então $(x, z) \in \mathcal{E}_{\preccurlyeq}$ (ou pela notação: se $x \preccurlyeq y$ e $y \preccurlyeq z$ então $x \preccurlyeq z)$;

As relações binárias que são anti-simétricas e transitivas são chamadas de relações de préordem e analogamente as relações binárias reflexivas, anti-simétricas e transitivas são chamadas de relações de ordem. Neste sentido, as Proposições 3.5, 3.6 e 3.7 respectivamente, garantem que a

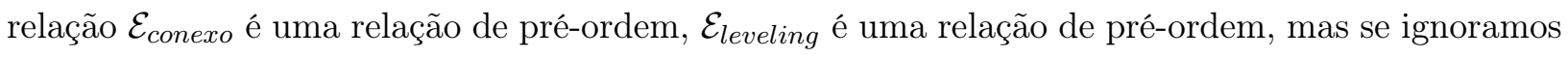
imagens constantes então $\mathcal{E}_{\text {leveling }}$ é uma relação de ordem e $\mathcal{E}_{\text {monotone planing é uma relação de }}$ pré-ordem. 


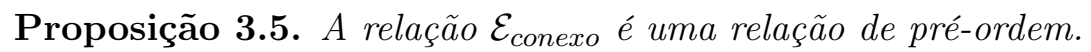

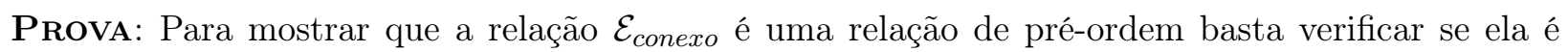
transitiva e reflexiva.

- transitiva: Sejam $f, g$ e $h \in \mathcal{F}(\mathcal{D})$ tais que $h$ é conexo de $g$ e $g$ é conexo de $f$. Logo, para $\forall(p, q) \in \mathcal{A}$, temos que, se $h(p) \neq h(q)$ então $g(p) \neq g(q)$. Consequentemente, temos que $f(p) \neq f(q)$, pois $g$ é conexo de $f$. Portanto, $h$ é conexo de $f$.

- reflexiva: Por definição, uma imagem $f \in \mathcal{F}(\mathcal{D})$ é conexa de si mesma.

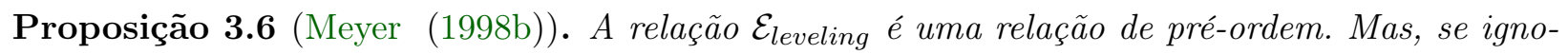
ramos imagens constantes então $\mathcal{E}_{\text {leveling }}$ é uma relação de ordem.

Prova: Para mostrar que $\mathcal{E}_{\text {leveling }}$ é uma relação de pré-ordem (respectivamente, ordem) basta verificar se ela é transitiva e reflexiva (respectivamente, anti-simétrica, transitiva e reflexiva).

- anti-simétrica: Sejam $g$ e $f \in \mathcal{F}(\mathcal{D})$ tais que $g$ é leveling de $f$ e $f$ é leveling de $g$. Logo, para $\forall(p, q) \in \mathcal{A}$, temos que:

se $g(p)>g(q)$ e $f(p)>f(q)$, então $f(p) \geq g(p), g(q) \geq f(q), g(p) \geq f(p)$ e $f(q) \geq g(q)$.

Como, $f(p) \geq g(p)$ e $g(p) \geq f(p)$ então $g(p)=f(p)$ e de forma análoga, temos que $g(q)=$ $f(q)$. Dessa forma, segue que $g(p)=f(p)$ e $g(q)=f(p)$. Portanto, como $g$ e $f$ não são constantes, então $g=f$ e consequentemente a relação $\mathcal{E}_{\text {leveling }}$ é anti-simétrica.

- transitiva: Sejam $f, g$ e $h \in \mathcal{F}(\mathcal{D})$ tais que $h$ é um leveling de $g$ e $g$ é um leveling de $f$. Logo, para $\forall(p, q) \in \mathcal{A}$, temos que: se $h(p)>h(q)$ então $g(p) \geq h(p)>h(q) \geq g(q)$. Como $g(p)>g(q)$ segue que $f(p) \geq g(p)>g(q) \geq f(q)$. Portanto, $h(p)>h(q) \Rightarrow g(p) \geq h(p)>$ $h(q) \geq g(q) \Rightarrow f(p) \geq g(p) \geq h(p)>h(p) \geq g(q) \geq f(q)$ o que mostra que $h$ é um leveling de $f$.

- reflexiva: Por definição, uma imagem $f \in \mathcal{F}(\mathcal{D})$ é leveling de si mesma, pois para $\forall(p, q) \in \mathcal{A}$, se $f(p)>f(q)$ então $f(p) \geq f(p)>f(q) \geq f(q)$.

Proposição 3.7 (Meyer (1998b)). A relação $\mathcal{E}_{\text {monotone planing é uma relação de pré-ordem. }}$

Prova: Para mostrar que a relação $\mathcal{E}_{\text {monotone planing }}$ é uma relação de pré-ordem basta verificar se ela é transitiva e reflexiva.

- transitiva: Sejam $f, g$ e $h \in \mathcal{F}(\mathcal{D})$ tais que $h$ é monotone planing de $g$ e $g$ é monotone planing de $f$. Logo, para $\forall(p, q) \in \mathcal{A}$, temos que, se $h(p)>h(q)$ então $g(p)>g(q)$. Consequentemente, temos que $f(p)>f(q)$, pois $g$ é monotone planing de $f$. Portanto, $h$ é monotone planing de $f$.

- reflexiva: Por definição, uma imagem $f \in \mathcal{F}(\mathcal{D})$ é monotone planing de si mesma. 


\subsection{Propriedades com relação as extremas regionais}

Os operadores monotone planing, como os levelings, além de preservar novos contornos da imagem também não criam uma outra estrutura da imagem, as extremas regionais. Em outras palavras, se $\mathcal{X}$ é uma extrema regional de um operador monotone planing, então existem extremas regionais na imagem referência contidas em $\mathcal{X}$, como pode ser observado pelo exemplo apresentado na Figura 3.5. Com base neste fato, Meyer (1998b) estabeleceu a Proposição 3.8.

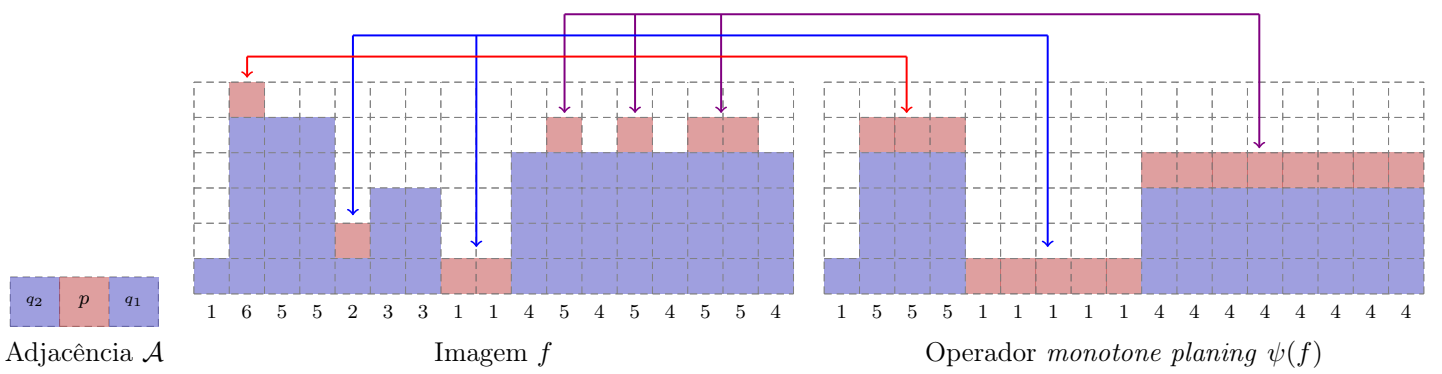

Figura 3.5: Ilustração destacando que os operadores monotone planing não criam novas extremas regionais.

Proposição 3.8 (Meyer (1998b)). Se g é um monotone planing de uma imagem $f$ possuindo uma minima (respectivamente, máxima) regional $\mathcal{X} \in \mathcal{Z P}(g)$, então $f$ possui uma mínima (respectivamente, máxima) regional $\mathcal{Z} \in \mathcal{Z} \mathcal{P}(f)$ incluída em $\mathcal{X}$, ou seja, $\mathcal{Z} \subseteq \mathcal{X}$.

Prova: Seja $\mathcal{X} \in \mathcal{Z P}(g)$ uma mínima regional de $g$ e considere $y=\arg \min _{x \in \mathcal{X}}\{f(x)\}$ um pixel de $\mathcal{X}$ de modo que $y$ tem um valor mínimo em $f$. Então, existe uma zona plana $\mathcal{Z}$ em $f$ que contém $y$. Claramente, $\mathcal{Z} \subseteq \mathcal{X}$, pois $g$ é conexo de $f$. Vamos mostrar, por contradição, que $\mathcal{Z} \subseteq \mathcal{X}$ é uma mínima regional em $f$.

Suponha que $\mathcal{Z}$ não é uma mínima regional de $f$. Então, existe um pixel $p \notin \mathcal{Z} \subseteq \mathcal{X}$ adjacente de algum pixel $q \in \mathcal{Z} \subseteq \mathcal{X}$, tal que $f(p)<f(q)=f(y)$. Como $g$ é um monotone planing de $f$, segue que, se $f(p) \leq f(q)$ então $g(p) \leq g(q)$, mas como $q \in \mathcal{X}$ e $\mathcal{X}$ é uma mínima regional de $g$, então $g(p)=g(q)$. Logo $p \in \mathcal{X}$, mas isso é um contradição e portanto $\mathcal{Z}$ é uma mínima regional de $f$.

\subsection{Algumas propriedades algébricas sobre os operadores conexos, levelings e monotone planings}

Nesta seção, são apresentadas algumas propriedades sobre derivação de operadores conexos, leveling e monotone planing. Essas propriedades são úteis no Capítulo 6, onde são estabelecidas as propriedades da classe dos últimos levelings.

Os operadores conexos (respectivamente, levelings e monotone planings) podem ser derivados por meio de combinações de operadores conexos (respectivamente, levelings e monotone planings). Assim, a composição de operadores conexos (respectivamente, levelings e monotone planings) é um operador conexo (respectivamente, levelings e monotone planings), da mesma forma que o ínfimo e o supremo de operadores conexos (respectivamente, levelings e monotone planings) são operadores conexos. Estas afirmações são garantidas pelas Proposições 3.9 a 3.14 .

Proposição 3.9 (Serra e Salembier (1993)). Se $\psi_{1}$ e $\psi_{2}$ são operadores conexos então, a composição $\psi_{1} \psi_{2}$ também é conexo.

Prova: Seja $f \in \mathcal{F}(\mathcal{D})$ e considere $(p, q) \in \mathcal{A}$. Então, por hipótese temos que $\psi_{1}$ e $\psi_{2}$ são operadores conexos. Logo, por definição: $\left[\psi_{2}\left(\psi_{1}(f)\right)\right](p) \neq\left[\psi_{2}\left(\psi_{1}(f)\right)\right](q) \Rightarrow\left[\psi_{1}(f)\right](p) \neq\left[\psi_{1}(f)\right](q)$ e 
consequentemente $\left[\psi_{1}(f)\right](p) \neq\left[\psi_{1}(f)\right](q) \Rightarrow f(p) \neq f(q)$. Portanto, por transitividade, temos que $\left[\psi_{2}\left(\psi_{1}(f)\right)\right](p) \neq\left[\psi_{2}\left(\psi_{1}(f)\right)\right](q) \Rightarrow f(p) \neq f(q)$, o que mostra que $\psi_{1} \psi_{2}$ é um operador conexo.

Proposição 3.10 (Meyer (1998b)). Se $\psi_{1}$ e $\psi_{2}$ são operadores levelings então, a composição $\psi_{1} \psi_{2}$ também é leveling.

Prova: Seja $f \in \mathcal{F}(\mathcal{D})$ e considere $(p, q) \in \mathcal{A}$. Então, por hipótese temos que $\psi_{1}$ e $\psi_{2}$ são operadores levelings. Logo, por definição: $\left[\psi_{2}\left(\psi_{1}(f)\right)\right](p)>\left[\psi_{2}\left(\psi_{1}(f)\right)\right](q) \Rightarrow\left[\psi_{1}(f)\right](p) \geq\left[\psi_{2}\left(\psi_{1}(f)\right)\right](p)>$ $\left[\psi_{2}\left(\psi_{1}(f)\right)\right](q) \geq\left[\psi_{1}(f)\right](q)$ e consequentemente $\left[\psi_{1}(f)\right](p)>\left[\psi_{1}(f)\right](q) \Rightarrow f(p) \geq\left[\psi_{1}(f)\right](p)>$ $\left[\psi_{1}(f)\right](q) \geq f(q)$. Portanto, por transitividade, temos que $\left[\psi_{2}\left(\psi_{1}(f)\right)\right](p)>\left[\psi_{2}\left(\psi_{1}(f)\right)\right](q) \Rightarrow$ $f(p) \geq\left[\psi_{2}\left(\psi_{1}(f)\right)\right](p)>\left[\psi_{2}\left(\psi_{1}(f)\right)\right](q) \geq f(q)$, o que mostra que $\psi_{1} \psi_{2}$ é um operador leveling.

Proposição 3.11. Se $\psi_{1}$ e $\psi_{2}$ são operadores monotone planing então, a composição $\psi_{1} \psi_{2}$ também é monotone planing.

Prova: Seja $f \in \mathcal{F}(\mathcal{D})$ e considere $(p, q) \in \mathcal{A}$. Então, por hipótese temos que $\psi_{1}$ e $\psi_{2}$ são operadores monotone planings. Logo, por definição: $\left[\psi_{2}\left(\psi_{1}(f)\right)\right](p)>\left[\psi_{2}\left(\psi_{1}(f)\right)\right](q) \Rightarrow\left[\psi_{1}(f)\right](p)>\left[\psi_{1}(f)\right](q)$ e consequentemente $\left[\psi_{1}(f)\right](p)>\left[\psi_{1}(f)\right](q) \Rightarrow f(p)>f(q)$. Portanto, por transitividade, temos que $\left[\psi_{2}\left(\psi_{1}(f)\right)\right](p)>\left[\psi_{2}\left(\psi_{1}(f)\right)\right](q) \Rightarrow f(p)>f(q)$, o que mostra que $\psi_{1} \psi_{2}$ é um operador monotone planing.

Proposição 3.12 (Serra e Salembier (1993)). Se $\left\{\psi_{0}, \psi_{1}, \ldots, \psi_{n}\right\}$ é uma família de operadores conexos então, o supremo $\bigvee_{0 \leq i \leq n} \psi_{i}$ e o ínfimo $\bigwedge_{0 \leq i \leq n} \psi_{i}$ são operadores conexos.

Prova: Seja $f \in \mathcal{F}(\mathcal{D})$ e considere $(p, q) \in \mathcal{A}$. Então, por hipótese temos que:

$$
f(p)=f(q) \Rightarrow\left[\psi_{0}(f)\right](p)=\left[\psi_{0}(f)\right](q),\left[\psi_{1}(f)\right](p)=\left[\psi_{1}(f)\right](q), \ldots,\left[\psi_{n}(f)\right](p)=\left[\psi_{n}(f)\right](q) .
$$

Claramente, $f(p)=f(q) \Rightarrow \bigvee_{0 \leq i \leq n} \psi_{i}(p)=\bigvee_{0 \leq i \leq n} \psi_{i}(q)$ e $\bigwedge_{0 \leq i \leq n} \psi_{i}(p)=\bigwedge_{0 \leq i \leq n} \psi_{i}(q)$. Portanto, o supremo $\bigvee_{0 \leq i \leq n} \psi_{i}$ e o ínfimo $\bigwedge_{0 \leq i \leq n} \psi_{i}$ são operadores conexos.

Proposição 3.13 (Meyer (1998b)). Se $\left\{\psi_{0}, \psi_{1}, \ldots, \psi_{n}\right\}$ é uma família de operadores levelings então, o supremo $\bigvee_{0 \leq i \leq n} \psi_{i}$ e o ínfimo $\bigwedge_{0 \leq i \leq n} \psi_{i}$ são operadores levelings.

Prova: Seja $f \in \mathcal{F}(\mathcal{D})$ e considere $(p, q) \in \mathcal{A}$. Por definição, se $\psi_{i}$ é um operador leveling então a seguinte relação é valida para todo $(p, q) \in \mathcal{A}$,

$$
f(p)<\left[\psi_{i}(f)\right](p) \leq\left[\psi_{i}(f)\right](q)<f(q) \Rightarrow\left[\psi_{i}(f)\right](p) \leq\left[\psi_{i}(f)\right](q) .
$$

Esta definição mostra que os valores de $p$ e $q$ em $\psi_{i}$ estão delimitados pelos valores de $f$. Assim, o ínfimo e o supremo da família $\left\{\psi_{0}, \psi_{1}, \ldots, \psi_{n}\right\}$ também estão delimitados pelos valores de $f$, pois esta família é de operadores leveling. Da mesma forma, a relação $\left[\psi_{i}(f)\right](p) \leq\left[\psi_{i}(f)\right](q)$ também é valida para o supremo e ínfimo desta família. Portanto, o ínfimo e o supremo de uma família de operadores levelings também é um operador leveling.

Proposição 3.14. Se $\left\{\psi_{0}, \psi_{1}, \ldots, \psi_{n}\right\}$ é uma família de operadores monotone planing então o supremo $\bigvee_{0 \leq i \leq n} \psi_{i}$ e o ínfimo $\bigwedge_{0 \leq i \leq n} \psi_{i}$ são operadores monotone planings. 
Prova: Seja $f \in \mathcal{F}(\mathcal{D})$ e considere $(p, q) \in \mathcal{A}$. Por definição, se $\psi_{i}$ é um operador monotone planing então a seguinte relação é valida para todo $(p, q) \in \mathcal{A}$,

$$
f(p) \leq f(q) \Rightarrow\left[\psi_{i}(f)\right](p) \leq\left[\psi_{i}(f)\right](q)
$$

Logo, como $\left[\psi_{0}(f)\right](p) \leq\left[\psi_{0}(f)\right](q),\left[\psi_{1}(f)\right](p) \leq\left[\psi_{1}(f)\right](q), \ldots,\left[\psi_{n}(f)\right](p) \leq\left[\psi_{n}(f)\right](q)$ e os operadores máximo e mínimo preservam esta ordem então $\bigvee_{0 \leq i \leq n}\left[\psi_{i}(f)\right](p) \leq \bigvee_{0 \leq i \leq n}\left[\psi_{i}(f)\right](q)$ e $\bigwedge_{0 \leq i \leq n}\left[\psi_{i}(f)\right](p) \leq \bigvee_{0 \leq i \leq n}\left[\psi_{i}(f)\right](q)$. Portanto, o ínfimo e o supremo de uma família de operadores monotone planings também são operadores monotone planings.

\subsection{Relações entre levelings e reconstruções por dilatações e erosões}

Por definição, um operador leveling $\psi$ aplicado em uma dada imagem $f \in \mathcal{F}(\mathcal{D})$ satisfaz duas inequações para cada par de transições entre pixels adjacentes $(p, q) \in \mathcal{A}$, isto é, quando houver uma transição entre $p$ e $q$ em $\psi$ então $f(p) \geq[\psi(f)](p)$ e $[\psi(f)](q) \geq f(q)$. Mais precisamente, um operador que satisfaz uma destas duas inequações é chamado de upper-leveling e lower-leveling e são apresentados pelas Definições 3.15 e 3.16.

Definição 3.15 (Operador upper-leveling (Meyer, 1998a)). Um operador $\psi: \mathcal{F}(\mathcal{D}) \rightarrow \mathcal{F}(\mathcal{D})$ é dito upper-leveling, se para toda imagem $f \in \mathcal{F}(\mathcal{D})$, a seguinte relação é válida para todos os pares de pixels adjacentes, ou seja, $\forall(p, q) \in \mathcal{A}$, temos que:

$$
\text { se }[\psi(f)](p)>[\psi(f)](q) \text { então } f(p) \geq[\psi(f)](p)
$$

Definição 3.16 (Operador lower-leveling (Meyer, 1998a)). Um operador $\psi: \mathcal{F}(\mathcal{D}) \rightarrow \mathcal{F}(\mathcal{D})$ é dito lower-leveling, se para toda imagem $f \in \mathcal{F}(\mathcal{D})$, a seguinte relação é válida para todos os pares de pixels adjacentes, ou seja, $\forall(p, q) \in \mathcal{A}$, temos que:

$$
\text { se }[\psi(f)](p)>[\psi(f)](q) \text { então }[\psi(f)](q) \geq f(q) \text {. }
$$

A reconstrução por dilatação $\delta_{\mathcal{B}}^{\infty}(f, g)$ de uma imagem $f \in \mathcal{F}(\mathcal{D})$ por um EE $\mathcal{B}$ e uma imagem marcadora $g \in \mathcal{F}(\mathcal{D})$, satisfaz a seguinte propriedade: $\delta_{\mathcal{B}}^{\infty}(f, g) \leq f$ e $\delta_{\mathcal{B}}^{\infty}(f, g)=\delta_{\mathcal{B}}\left(\delta_{\mathcal{B}}^{\infty}(f, g)\right) \wedge f$. Esta propriedade também é verificada pelos lower-levelings que são anti-extensivos, como observado por Meyer (1998a) na Proposição 3.17.

Proposição 3.17 (Meyer (1998a)). Um operador $\psi$ é lower-leveling (respectivamente, upperleveling) e anti-extensivo (respectivamente, extensivo) se, e somente se, $\psi$ é uma reconstrução por dilatação (respectivamente, erosão).

Prova: Considere $g=\psi(f)$ para uma dada imagem $f \in \mathcal{F}(\mathcal{D})$. Como $\psi$ é lower-leveling e antiextensivo, segue que: $g \leq f$ e $g$ é lower-leveling de $f$.

$\Longleftrightarrow g \leq f$ e $\forall(p, q) \in \mathcal{A}, g(p)>g(q) \Rightarrow g(q) \geq f(q)$.

$\Longleftrightarrow g \leq f$ e $\forall(p, q) \in \mathcal{A}, g(p) \leq g(q)$ ou $g(q) \geq f(q)$. [pelo argumento lógico: $A \Rightarrow B \Longleftrightarrow B$ ou $\neg A$ ]

$\Longleftrightarrow g \leq f$ e $\forall(p, q) \in \mathcal{A}, g(q) \geq g(p) \wedge f(q)$.

$\Longleftrightarrow g \leq f$ e $\forall q \in \mathcal{D}, p \in \mathcal{A}(q), g(q) \geq g(p) \wedge f(q)$.

$\Longleftrightarrow g \leq f$ e $\forall q \in \mathcal{D}, g(q) \geq \bigvee_{p \in \mathcal{A}(q)}\{g(p)\} \wedge f(q)$

$\Longleftrightarrow g \leq f$ e $\forall q \in \mathcal{D}, g(q) \geq\left[\delta_{\mathcal{B}}(g)\right](q) \wedge f(q)$, onde $(p, q) \in \mathcal{A} \Longleftrightarrow q \in(\mathcal{B}+p)$.

$\Longleftrightarrow g \leq f$ e $g=\delta_{\mathcal{B}}^{\infty}(f, g)$ é uma reconstrução por dilatação de uma imagem $f$ por EE simétrico e 
isotrópico $\mathcal{B}$ e alguma imagem marcadora $g \in \mathcal{F}(\mathcal{D})$.

Analogamente, pode-se provar que upper-levelings são reconstruções por erosões.

Além dessas relações entre lower-levelings e upper-levelings com reconstruções por dilatações e erosões, é possível, seguindo Meyer (1998b) na Equação 3.10, construir um operador leveling auto-dual $\rho$ combinando as reconstruções por dilatação e erosão.

$$
\forall p \in \mathcal{D},[\rho(f, g)](p)= \begin{cases}{\left[\delta_{\mathcal{B}}^{\infty}(f, g \wedge f)\right](p)} & , \text { se } g(p)<f(p), \\ {\left[\varepsilon_{\mathcal{B}}^{\infty}(f, g \vee f)\right](p)} & \text {, se } g(p)>f(p), \\ f(p) & \text {, caso contrário. }\end{cases}
$$

Também é muito comum construir operadores levelings por meio de composição, por exemplo: $\varepsilon_{\mathcal{B}}^{\infty}\left(\delta_{\mathcal{B}}^{\infty}(f, g \wedge f), g \vee f\right)$ e $\delta_{\mathcal{B}}^{\infty}\left(\varepsilon_{\mathcal{B}}^{\infty}(f, g \vee f), g \wedge f\right)$. Na Figura 3.6 são apresentados exemplos de construções de levelings a partir de imagens marcadoras.

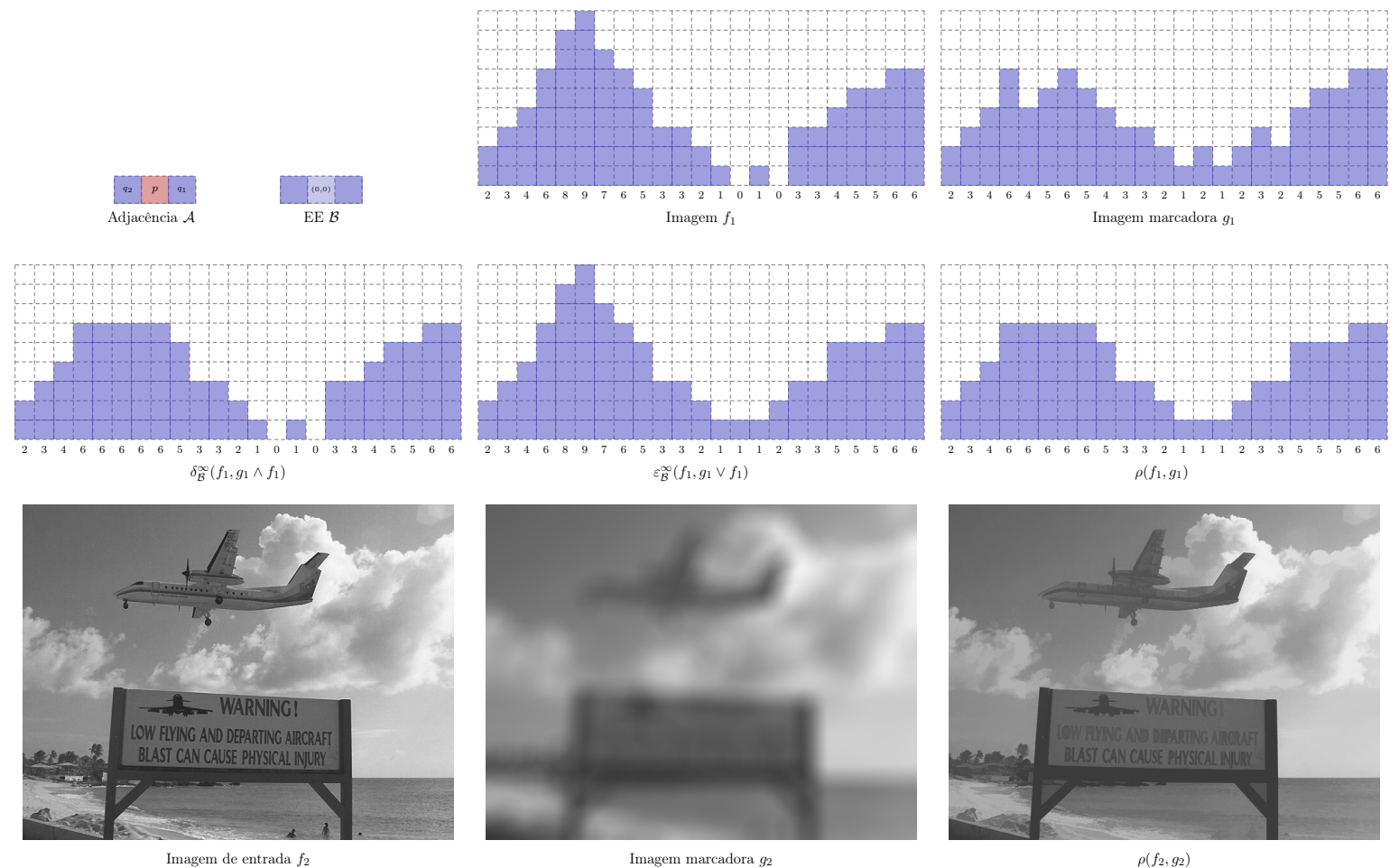

Figura 3.6: Exemplos de construções de levelings a partir de imagens marcadoras.

\subsection{Espaço de escala com base em levelings}

Como mostrado na Seção 3.2, a relação binária $\mathcal{E}_{\text {leveling }}$ é reflexiva e transitiva e, se ignoramos as imagens constantes, então a relação também é anti-simétrica, ou seja, $\mathcal{E}_{\text {leveling }}$ é uma relação de ordem quando ignoram-se as imagens constantes. Com a ajuda desta relação, os levelings podem ser aninhados para criar um espaço de escala de uma dada imagem $f \in \mathcal{F}(\mathcal{D})$ em forma de uma sequência de levelings $\left(g_{0}=f, g_{1}, \ldots, g_{n}\right)$, onde $g_{k}$ é leveling de $g_{k-1}$ e, por consequência da transitividade, $g_{k}$ também é leveling de cada imagem $g_{j}$, para todo $j<k$. Neste sentido, o tradicional algoritmo para construir levelings, descrito pela Equação 3.10, toma como argumento duas imagens, uma imagem de entrada $f \in \mathcal{F}(\mathcal{D})$ e uma imagem marcadora $h \in \mathcal{F}(\mathcal{D})$. Ele reconstrói a imagem marcadora $h$ de tal maneira a torná-la leveling da imagem $f$. Assim, dizemos que $g=\rho(f, h)$ é leveling de $f$, obtida a partir do marcador $h$. Com este operador é possível construir um espaço 
de escala baseado em levelings a partir de qualquer família $\left(h_{1}, h_{2}, \ldots, h_{n}\right)$ de imagens marcadoras com o seguinte encadeamento: $g_{0}=f, g_{1}=\rho\left(g_{0}, h_{1}\right), g_{2}=\rho\left(g_{1}, h_{2}\right), \ldots, g_{n}=\rho\left(g_{n-1}, h_{n}\right)$. Esta sequência de levelings simplificam mais e mais a imagem $f$ e, como mostrado por Meyer e Maragos (2000), constitui um espaço de escala com as seguintes características:

1. Simplificação: A partir de uma escala para a outra, uma simplificação real acontece, ou seja, se $g_{i+1}$ é leveling de $g_{i}$ então todos os contornos e todas as extremas regionais de $g_{i+1}$ estão presentes em $g_{i}$.

2. Princípio do máximo: Uma forma particular de simplificação é expressa pelo princípio do máximo: qualquer mudança de escala, a intensidade máxima em escalas grosseiras é sempre menor do que a intensidade máxima das escalas finas, isto é, se $g_{i+1}$ é um leveling de $g_{i}$ então qualquer transição entre duas regiões conexas de $g_{i+1}$ é delimitada por uma grande variação entre os níveis de cinza destas regiões em $g_{i}$

3. Princípio da causalidade: O que acontece em escalas mais grosseiras só pode ser causado pelo que aconteceu em escalas mais finas, ou melhor, se $g_{i+1}$ é leveling de $g_{i}$ então todos os contornos presentes em $g_{i+1}$ correspondem a contornos mais fortes em $g_{i}$ nos mesmos lugares e também as extremas regionais em $g_{i+1}$ estão presentes em $g_{i}$.

4. Fidelidade: Não criar novas estruturas em escalas mais grosseiras: cada mínima (respectivamente, máxima) regional do leveling contém uma mínima (respectivamente, máxima) regional da imagem de referência. Além das estruturas que são reprimidas, todos os outros contornos são preservados sem qualquer deslocamento ou desfocagem (ver Definição 3.2 e Proposição 3.8).

5. Invariâncias: Os levelings são invariantes às transformações de rotação (isotropia), translação espacial e mudança de iluminação, isto é, como a relação de adjacência é simétrica têm-se as invariâncias por rotação e translação, e no caso da invariância a iluminação, é sabido que se $g_{i+1}$ é leveling de $g_{i}$ e, $g_{i}$ e $g_{i+1}$ são submetidos a uma transformação linear $\psi$ então $\psi\left(g_{i+1}\right)$ ainda é leveling de $\psi\left(g_{i}\right)$.

Na Figura 3.7 são apresentados exemplos de imagens de um espaço de escala baseado em levelings construídos a partir de uma família de marcadores compostos por filtros medianos.

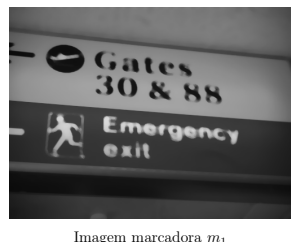

Imagem marcadora $m_{1}$

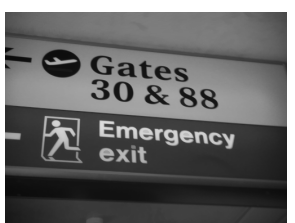

Leveling $g_{1}=\rho\left(f, m_{1}\right)$

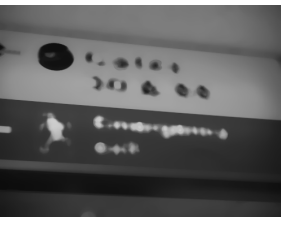

Imagem marcadora $m_{2}$

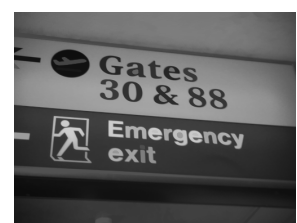

Leveling $g_{2}=\rho\left(g_{1}, m_{2}\right)$

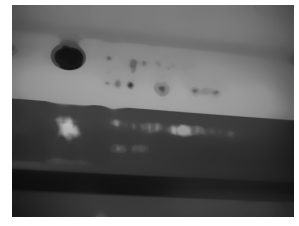

Imagem marcadora $m_{3}$

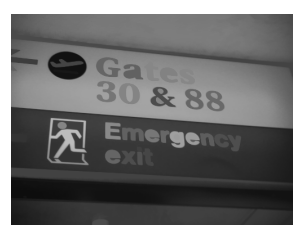

Leveling $g_{3}=\rho\left(g_{2}, m_{3}\right)$

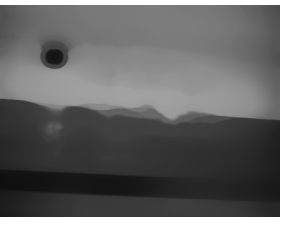

Imagem marcadora $m_{4}$

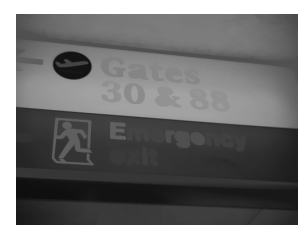

Leveling $g_{4}=\rho\left(g_{3}, m_{4}\right)$

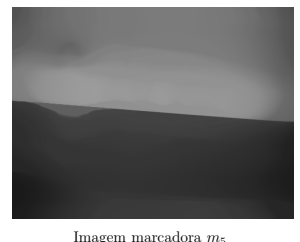

Imagem marcadora $m_{5}$

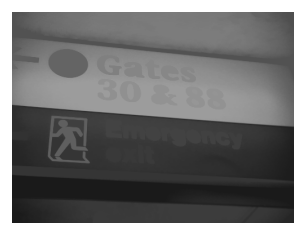

Leveling $g_{5}=\rho\left(g_{4}, m_{5}\right)$

Figura 3.7: Exemplos de imagens de um espaço de escala baseado em levelings construído a partir de uma familia de marcadores compostos por filtros medianos. 


\section{Representações de imagens por meio de árvores baseadas em conjuntos de níveis}

\section{Resumo do capítulo}

Representações de imagens por meio de árvores têm sido propostas nos últimos anos para realizar tarefas de análise e processamento de imagens, como por exemplo: filtragem, segmentação, reconhecimento de padrões, extração de contraste, registro, compactação e outras. Neste cenário, como ilustrado na Figura 4.1, o primeiro passo para realização da tarefa é construir uma representação da imagem por meio de uma árvore, depois a tarefa de processamento e análise de imagens é realizada através de modificações ou extrações de informações na própria árvore e finalmente uma imagem é reconstruída a partir da árvore modificada ou das informações extraídas da árvore. Neste capítulo

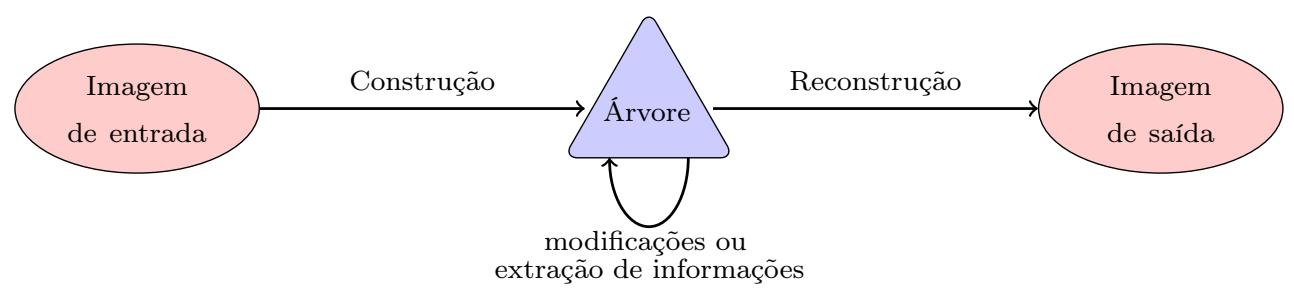

Figura 4.1: Representação de uma imagem por meio de árvore.

são apresentados algoritmos e modelos de árvores construídas a partir dos conjuntos de niveis de uma decomposição da imagem por limiarização. Estes modelos incluem as árvores de componentes e as árvores de formas. Essas árvores, como mostrado no Capítulo 5, têm uma forte relação com operadores levelings.

\subsection{Conceitos de árvores construídas por conjuntos parcialmente ordenados}

Como apresentado na Seção 3.2, uma relação binária $\mathcal{E}_{\preccurlyeq}$ sobre um conjunto $\mathcal{V}$ é chamada de relação de ordem se, e somente se, ela é transitiva, reflexiva e anti-simétrica. Neste caso, o par $(\mathcal{V}, \preccurlyeq)$ é conhecido por conjunto ordenado, ou seja, $\mathcal{V}$ é um conjunto ordenado pela relação $\preccurlyeq$. Mais especificamente, se quaisquer dois elementos de $a, b \in \mathcal{V}$ sempre são comparáveis $(a \preccurlyeq b$ ou $b \preccurlyeq a)$, então o par $(\mathcal{V}, \preccurlyeq)$ é chamado de conjunto totalmente ordenado, caso contrário, chama-se o par $(\mathcal{V}, \preccurlyeq)$ de conjunto parcialmente ordenado (poset - do inglês, partially ordered set).

$\operatorname{Um}$ poset $(\mathcal{V}, \preccurlyeq)$ pode ser representado por um grafo não-direcionado $\mathcal{G}=\left(\mathcal{V}, E_{\preccurlyeq}\right)$ conhecido 
por Diagrama de Hasse, onde seus vértices são os elementos do conjunto $\mathcal{V}$ e suas arestas $E_{\preccurlyeq}$ são definidas pela seguinte propriedade: $\{v, w\}$ é uma aresta em $E_{\preccurlyeq}$ se, e somente se, $v, w \in \mathcal{V}, v \neq w$, $v$ e $w$ são comparáveis e não existe $x \in \mathcal{V}$ diferente de $v$ e $w$ tal que ou $v \preccurlyeq x \preccurlyeq w$, ou $w \preccurlyeq x \preccurlyeq v$. Assim, posets finitos podem ser desenhados (diagrama de Hasse) da seguinte forma: os vértices de $\mathcal{V}$ são representados por pequenos círculos dispostos de tal forma que um circulo $v$ está acima de um circulo $w$, se $\{v, w\}$ é uma aresta em $E_{\preccurlyeq}$ satisfazendo $w \preccurlyeq v$ e neste caso, desenha-se um segmento de reta de $v$ para $w$. Na Figura 4.2 é apresentado um diagrama de Hasse para um dado poset.

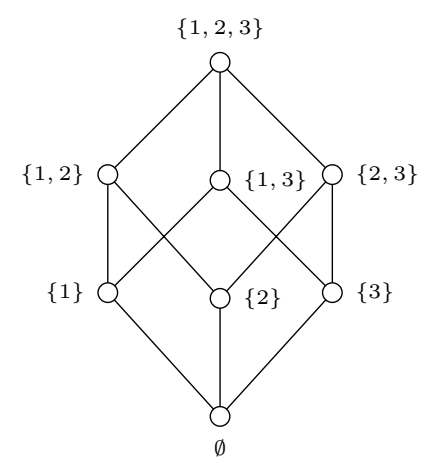

Figura 4.2: Exemplo do diagrama de Hasse do poset $(\{\emptyset,\{1\},\{2\},\{3\},\{1,2\},\{1,3\},\{2,3\},\{1,2,3\}\}, \subseteq)$.

Como é conhecido, uma árvore é um grafo conexo e sem ciclos. Neste sentido, o grafo $\mathcal{G}=\left(\mathcal{V}, E_{\preccurlyeq}\right)$ do diagrama de Hasse é uma árvore se, e somente se, o poset $(\mathcal{V}, \preccurlyeq)$ satisfaz as duas condições da Definição 4.1.

Definição 4.1 (Árvore). Um grafo do diagrama de Hasse de um poset ( $\mathcal{V}, \preccurlyeq)$ é uma árvore se, e somente se, as duas condições a seguir são verificadas e, neste caso, o poset $(\mathcal{V}, \preccurlyeq)$ é chamado simplesmente por árvore.

1. $\exists R \in \mathcal{V}$ tal que $\forall N \in \mathcal{V}, N \preccurlyeq R$. Neste caso, é dito que $R$ é a raiz da árvore.

2. $\forall A, B, C \in \mathcal{V}$, se $A \preccurlyeq B$ e $A \preccurlyeq C$ então ou $B \preccurlyeq C$, ou $C \preccurlyeq B$. Assim, é dito que $B$ e $C$ estão aninhados (ou são comparáveis).

A primeira condição expressa a conexidade do poset, sendo $R$ a raiz da árvore, e a segunda condição implica que não há ciclo, pois sejam $A, B, C, D \in \mathcal{V}$, a seguinte situação nunca acontece: $A \preccurlyeq B \preccurlyeq D$ e $A \preccurlyeq C \preccurlyeq D$ e $B$ e $C$ não comparáveis, como pode ser facilmente observado no diagrama de Hasse da Figura 4.3.

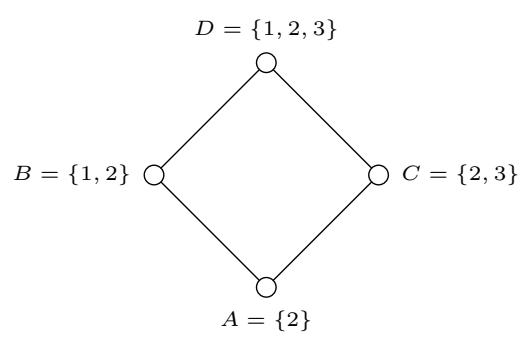

Figura 4.3: Exemplo do diagrama de Hasse do poset $(\{\{2\},\{1,2\},\{2,3\}\{1,2,3\}\}, \subseteq)$ que não satisfaz a Definição 4.1.

Na Figura 4.4 é apresentado um diagrama de Hasse para um dado poset que satisfaz a Definição 4.1 e, em seguida, são apresentadas algumas definições usuais sobre uma árvore $\mathcal{T}=(\mathcal{V}, \preccurlyeq)$. 


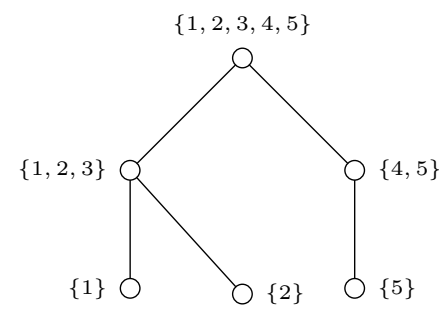

Figura 4.4: Exemplo do diagrama de Hasse do poset $\mathcal{T}=(\{\{1\},\{2\},\{5\},\{1,2,3\},\{4,5\},\{1,2,3,4,5\}\}, \subseteq)$ que satisfaz a Definição 4.1.

Definição 4.2 (Raiz). O vértice $R \in \mathcal{V}$ é chamado por raiz da árvore $\mathcal{T}$ se, e somente se, $\forall A \in$ $\mathcal{V}, A \preccurlyeq R$. Neste caso, denota-se por $\operatorname{Raiz}(\mathcal{T})$ o vértice $R$ raiz da árvore $\mathcal{T}$.

Definição 4.3 (Pai). O vértice $B \in \mathcal{V}$ é chamado de pai do vértice $A \in \mathcal{V}$ se, e somente se, $A \preccurlyeq B$ e não existir um vértice $C \in \mathcal{V}$ diferente de $A$ e $B$ tal que $A \preccurlyeq C \preccurlyeq B$. Neste caso, denota-se por $\operatorname{Pai}(A)$ o vértice $B$ pai do vértice $A$.

Definição 4.4 (Filhos). Os vértices da árvore $\mathcal{T}$ que tem como pai um vértice $A \in \mathcal{V}$ são chamados de filhos do vértice $A$ e definido pelo conjunto $\operatorname{Filhos}(A)=\{B \in \mathcal{V}: \operatorname{Pai}(B)=A\}$.

Definição 4.5 (Folha). Um vértice $A \in \mathcal{V}$ é chamado de folha se, e somente se, o vértice $A$ não possui filhos, ou seja, $\mathrm{Filhos}(A)=\emptyset$. Assim, o conjunto das folhas da árvore $\mathcal{T}$ é definido pelo conjunto $\operatorname{Folhas}(\mathcal{T})=\{A \in \mathcal{V}: \operatorname{Filhos}(A)=\emptyset\}$

Definição 4.6 (Ascendente e Descendente). Sejam $A, B \in \mathcal{V}$ tal que $A \preccurlyeq B$. Neste caso, é dito que $B$ é ascendente de $A$, ou ainda, que $A$ é descendentes de $B$.

Definição 4.7 (Intervalo). Sejam $A, B \in \mathcal{V}$ tal que $A \preccurlyeq B$. Então, chama-se $[A, B]_{\mathcal{T}}$ o intervalo entre $A$ e $B$ se, e somente se, $[A, B]_{\mathcal{T}}=\{S \in \mathcal{V}: A \preccurlyeq S \preccurlyeq B\}$.

Definição 4.8 (Bifurcação). Sejam $A, B \in \mathcal{V}$ tal que $A \preccurlyeq B$. Então, diz-se que existe uma bifurcação entre $A$ e $B$, se existir um $S \in \mathcal{V}$ tal que $S \preccurlyeq B$ e $S$ não é comparável a $A$.

Definição 4.9 (Ramo). Sejam $A, B \in \mathcal{V}$ tal que $A \preccurlyeq B$. Então, diz-se que $[A, B]_{\mathcal{T}}$ é um ramo da árvore $\mathcal{T}$, se não existir uma bifurcação entre $A$ e $B$.

A seguir são apresentados exemplos simples de aplicações das Definições 4.3 a 4.9 no poset $\mathcal{T}$ ilustrado na Figura 4.4.

1. A raiz da árvore $\mathcal{T}$ é o vértice $\operatorname{Raiz}(\mathcal{T})=\{1,2,3,4,5\}$;

2. As folhas da árvore $\mathcal{T}$ são os vértices do conjunto $\operatorname{Folhas}(\mathcal{T})=\{\{1\},\{2\},\{5\}\}$;

3. O pai do vértice $\{2\}$ é o conjunto Pai $(\{2\})=\{1,2,3\}$;

4. Os filhos do vértice $\{1,2,3\}$ é o conjunto Filhos $(\{1,2,3\})=\{\{1\},\{2\}\}$;

5. O intervalo entre os vértices $\{1\}$ e $\{1,2,3,4,5\}$ é o conjunto

$[\{1\},\{1,2,3,4,5\}]_{\mathcal{T}}=\{\{1\},\{1,2,3\},\{1,2,3,4,5\}\} ;$

6. Existe uma bifurcação no intervalo $[\{1\},\{1,2,3,4,5\}]_{\mathcal{T}}$; 
7. O intervalo $[\{5\},\{1,2,3,4,5\}]_{\mathcal{T}}$ é um ramo.

A partir de uma árvore $\mathcal{T}$, pode-se definir uma operação essencial neste trabalho denominada poda. Esta operação produz uma árvore por meio de eliminações de vértices e arestas da árvore $\mathcal{T}$. Assim, é dito que uma árvore $\mathcal{T}^{\prime}$ é obtida por uma operação de poda sobre a árvore $\mathcal{T}$ se a Definição 4.10 é verdadeira.

Definição 4.10 (Poda). Diz-se que um árvore $\mathcal{T}^{\prime}=\left(\mathcal{V}^{\prime}, \preccurlyeq\right)$ é obtida por uma operação de poda sobre a árvore $\mathcal{T}=(\mathcal{V}, \preceq)$ se, e somente se, $\mathcal{V}^{\prime} \subseteq \mathcal{V}$ e qualquer $A \in \mathcal{V}^{\prime}, \nexists B \in\left(\mathcal{V} \backslash \mathcal{V}^{\prime}\right)$ tal que $A \preccurlyeq B$. Neste caso, a operação de poda é denotada por $\mathcal{T}^{\prime}=\mathcal{P}$ oda $(\mathcal{T})$.

Vale salientar $\mathcal{T}^{\prime}$ é uma árvore, isto é, satisfaz a Definição 4.1. Na Figura 4.5 é apresentado um exemplo de operação de poda na árvore ilustrada na Figura 4.4.

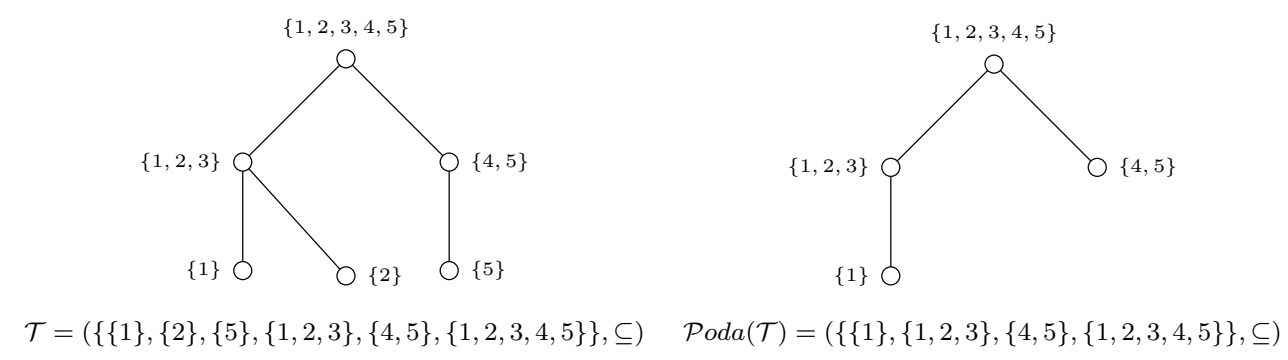

Figura 4.5: Exemplo de operação de poda para uma dada árvore.

\section{2 Árvores baseadas em conjuntos de níveis}

O conjunto de pixels de $f$ que estão estritamente abaixo (respectivamente, acima) de um determinado nível de cinza $h \in \mathbb{K}$ é denominado de conjunto de nível inferior de valor $h$ (respectivamente, conjunto de nível superior de valor $h$. Este conjunto também é conhecido como cross-section de $f$ de valor $h$ (Serra, 1982)). Mais precisamente, os conjuntos de níveis inferiores e superiores são definidos da seguinte forma:

Definição 4.11 (Conjunto de nível). O conjunto de nível inferior $\mathcal{X}_{\downarrow}^{\lambda}(f)$ e o conjunto de nível superior $\mathcal{X}_{\lambda}^{\uparrow}(f)$ de valor $\lambda \in \mathbb{K}$ para uma imagem $f \in \mathcal{F}(\mathcal{D})$, são definidos por:

$$
\mathcal{X}_{\downarrow}^{\lambda}(f)=\{p \in \mathcal{D}: f(p)<\lambda\} \text { e } \mathcal{X}_{\lambda}^{\uparrow}(f)=\{p \in \mathcal{D}: f(p) \geq \lambda\}
$$

A convenção por tomar a desigualdade estrita para conjuntos de nível inferior e não-estrita para conjuntos de nível superior é para obter resultados de consistência entre eles, como por exemplo, $\mathcal{D} \backslash \mathcal{X}_{\lambda}(f)=\mathcal{X}^{\lambda}(f)$. Além de não misturá-los quando lidarmos com ambos simultaneamente.

Estes conjuntos são aninhados pela relação de inclusão. Mais precisamente, o conjunto de nível inferior é crescente, isto é

$$
\mathcal{X}_{\downarrow}^{1}(f) \subseteq \mathcal{X}_{\downarrow}^{2}(f) \subseteq \ldots \subseteq \mathcal{X}_{\downarrow}^{K}(f),
$$

e o conjunto de nível superior é decrescente, ou seja

$$
\mathcal{X}_{K}^{\uparrow}(f) \subseteq \mathcal{X}_{K-1}^{\uparrow}(f) \subseteq \ldots \subseteq \mathcal{X}_{0}^{\uparrow}(f)
$$

Consequentemente, $\forall \alpha, \beta \in \mathbb{K}$, tal que $\alpha<\beta$ segue que $\mathcal{X}_{\downarrow}^{\alpha}(f) \subseteq \mathcal{X}_{\downarrow}^{\beta}(f)$ e $\mathcal{X}_{\alpha}^{\uparrow}(f) \supseteq \mathcal{X}_{\beta}^{\uparrow}(f)$. Outro fato interessante é que se $q \in \mathcal{D}$, então $\mathcal{X}_{\downarrow}^{f(q)+1}(f)=\{p \in \mathcal{D}: f(p)<f(q)+1\}$ é o conjunto de nível 
inferior de menor valor que contém $q$, pois $\mathcal{X}_{\downarrow}^{f(q)+1}(f) \subseteq \mathcal{X}_{\downarrow}^{f(q)+2}(f) \subseteq \ldots \mathcal{X}_{\downarrow}^{K}(f)$. Logo, o nível de cinza $f(q)=\min \left\{\lambda-1: q \in \mathcal{X}_{\downarrow}^{\lambda}(f), \lambda \in \mathbb{K}\right\}$. Analogamente, $\mathcal{X}_{f(q)}^{\uparrow}(f)=\{p \in \mathcal{D}: f(p) \geq f(q)\}$ é o conjunto de nível superior de maior valor que contém $q$. Assim, o nível de cinza $f(q)=\max \{\lambda$ : $\left.q \in \mathcal{X}_{\lambda}^{\uparrow}(f), \lambda \in \mathbb{K}\right\}$. Portanto, isso mostra que basta uma família de conjuntos de níveis inferiores ou superiores extraídos de uma imagem $f$, para reconstruir esta imagem $f$, isto é,

$$
\forall p \in \mathcal{D}, f(p)=\min \left\{\lambda-1: p \in \mathcal{X}_{\downarrow}^{\lambda}(f), \lambda \in \mathbb{K}\right\}=\max \left\{\lambda: p \in \mathcal{X}_{\lambda}^{\uparrow}(f), \lambda \in \mathbb{K}\right\}
$$

Na Figura 4.6 é apresentada uma imagem de entrada com seus respectivos conjuntos de níveis superiores e inferiores.
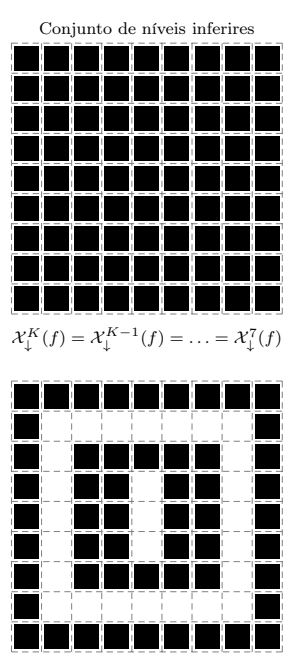

$\mathcal{X}_{\downarrow}^{6}(f)=\mathcal{X}_{\downarrow}^{5}(f)=\mathcal{X}_{\downarrow}^{4}(f)$

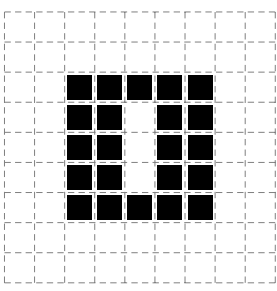

$\mathcal{X}_{\downarrow}^{3}(f)=\mathcal{X}_{\downarrow}^{2}(f)$

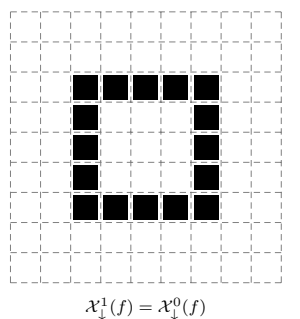

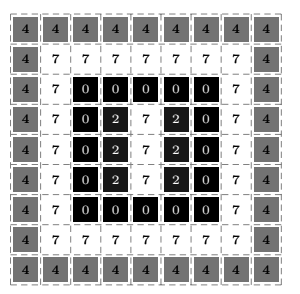

imagem $f$
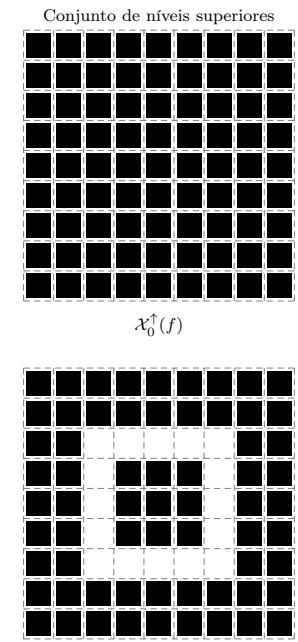

$\mathcal{X}_{1}^{\uparrow}(f)=\mathcal{X}_{2}^{\uparrow}(f)$

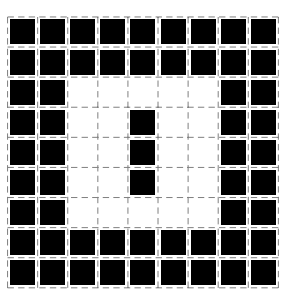

$\mathcal{X}_{3}^{\uparrow}(f)=\mathcal{X}_{4}^{\uparrow}(f)$

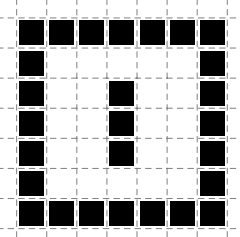

$\mathcal{X}_{5}^{\uparrow}(f)=\mathcal{X}_{6}^{\uparrow}(f)=\mathcal{X}_{7}^{\uparrow}(f)$

Figura 4.6: Exemplo de conjuntos de níveis extraídos de uma imagem. Na parte de cima central está a imagem de entrada $f$ e as colunas da esquerda e da direita, respectivamente, estão os conjuntos de níveis $\mathcal{X}_{\downarrow}^{\lambda}(f)$ e $\mathcal{X}_{\lambda}^{\uparrow}(f)$. 


\subsection{1 Ärvore de componentes}

Uma árvore de componentes é uma estrutura hierárquica de CCs dos conjuntos de níveis extraídos de uma imagem. Ela pode ser obtida pelos conjuntos de níveis superiores ou inferiores e tem sido usada, sob diversas variações, em um grande número de aplicações entre as quais podese citar: filtragem (Jones, 1999; Najman e Couprie, 2006), segmentação (Couprie e Bertrand, 1997; Silva e de Alencar Lotufo, 2011), extração de resíduos (Alves e Hashimoto, 2014; Alves et al., 2013; Fabrizio e Marcotegui, 2009), registro (Mattes et al., 1999) e compressão (Salembier et al., 1998). Além disso, a estrutura desta árvore é fundamental para a computação eficiente de outros métodos, como por exemplo, o watershed topológico (Couprie e Bertrand, 1997; Couprie et al., 2005), a árvore de zonas quasi-planas (Soille e Najman, 2012) e regiões extremais maximamente estáveis (Matas et al., 2004).

Para definirmos a árvore de componentes, considere $\mathcal{L}(f)$ a família dos CCs dos conjuntos de níveis inferiores de $f$, ou seja, $\mathcal{L}(f)=\left\{C: C \in \mathcal{C C}\left(\mathcal{X}_{\downarrow}^{\lambda}(f), \mathcal{A}\right), \lambda \in \mathbb{K}\right\}$ e considere também $\mathcal{U}(f)$ a família de CCs dos conjunto de níveis superiores de $f$, isto é, $\mathcal{U}(f)=\left\{C: C \in \mathcal{C C}\left(\mathcal{X}_{\lambda}^{\uparrow}(f), \mathcal{A}\right), \lambda \in \mathbb{K}\right\}$. Observe que, por definição dos conjunto de níveis, se $\alpha \geq \beta$ e $A \in \mathcal{C C}\left(\mathcal{X}_{\alpha}^{\uparrow}(f), \mathcal{A}\right), B \in \mathcal{C C}\left(\mathcal{X}_{\beta}^{\uparrow}(f), \mathcal{A}\right)$, então ou $A \cap B=\emptyset$ ou $A \subseteq B$, pois se existir intersecção entre $A$ e $B$, então existem caminhos de $A$ para $B$ em $B$, já que $B$ é uma $\mathrm{CC}$ e com isso $A \subseteq B$. De maneira similar, se $A \in \mathcal{C C}\left(\mathcal{X}_{\downarrow}^{\alpha}(f), \mathcal{A}\right)$, $B \in \mathcal{C C}\left(\mathcal{X}_{\downarrow}^{\beta}(f), \mathcal{A}\right)$ então ou $A \cap B=\emptyset$ ou $B \subseteq A$. Então, o poset consistindo da família das CCs dos conjuntos dos níveis inferiores (respectivamente, superiores) e a usual relação de inclusão leva à árvore de componentes dos conjuntos de níveis inferiores (respectivamente, superiores). Assim, a Proposição 4.12 garante que os posets $(\mathcal{L}(f), \subseteq)$ e $(\mathcal{U}(f), \subseteq)$ são árvores.

Proposição 4.12. Ambos os posets $(\mathcal{L}(f), \subseteq)$ e $(\mathcal{U}(f), \subseteq)$ são árvores.

Prova: Para mostrar que $(\mathcal{L}(f), \subseteq)$ e $(\mathcal{U}(f), \subseteq)$ são árvores basta verificar se a Definição 4.1 é satisfeita. Na sequência, é mostrado que $(\mathcal{L}(f), \subseteq)$ satisfaz as duas condições da Definição 4.1.

1. Seja $\lambda$ um nível a mais do que o maior nível de cinza de $f$. Por definição, $\mathcal{X}_{\downarrow}^{\lambda}(f)=\{x \in \mathcal{D}$ : $f(x)<\lambda\}$. Logo, todos os pixels $x \in \mathcal{D}$ satisfazem $f(x)<\lambda$, pois $\lambda$ é maior do que todos os níveis de cinzas de $f$. Como $\mathcal{D}$ é um subconjunto retangular e compacto de $\mathbb{Z}^{2}$ então $\mathcal{D}$ é uma $\mathrm{CC}$ e consequentemente $\mathcal{D} \in \mathcal{U}(f)$. Portanto, $\forall A \in \mathcal{U}(f)$ temos que $A \subseteq \mathcal{D}$.

2. Sejam $A, B, C \in \mathcal{L}(f)$ tais que $A \subseteq B$ e $A \subseteq C$. Então, existem inteiros $a, b$ e $c$ tais que $A \in \mathcal{C C}\left(\mathcal{X}_{\downarrow}^{a}(f)\right), B \in \mathcal{C C}\left(\mathcal{X}_{\downarrow}^{b}(f)\right)$ e $C \in \mathcal{C C}\left(\mathcal{X}_{\downarrow}^{c}(f)\right)$. Considere $p \in A$. Então, como $A \subseteq B$ e $A \subseteq C$ seguem:

(a) $p \in B$ e $a \leq b$, pois $A \subseteq \mathcal{X}_{\downarrow}^{a}(f)=\{q \in \mathcal{D}: f(q)<a \leq b\} \subseteq \mathcal{X}_{\downarrow}^{b}(f)=\{q \in \mathcal{D}: f(q)<b\}$;

(b) $p \in C$ e $a \leq c$, pois $A \subseteq \mathcal{X}_{\downarrow}^{a}(f)=\{q \in \mathcal{D}: f(q)<a \leq c\} \subseteq \mathcal{X}_{\downarrow}^{c}(f)=\{q \in \mathcal{D}: f(q)<c\}$;

(c) Portanto, por $(a)$ e $(b)$ temos $p \in B \cap C$.

Logo, como $B \cap C \neq \emptyset$ então, ou $c \leq b$, ou $b \leq c$.

- Se $c \leq b$, então $C \subseteq \mathcal{X}_{\downarrow}^{c}(f)=\{q \in \mathcal{D}: f(q)<c \leq b\} \subseteq \mathcal{X}_{\downarrow}^{b}(f)$. Logo, como $B$ é uma $\mathrm{CC}$, então existem caminhos em $B$ para todos os pixels de $C$, pois $B \cap C \neq \emptyset$. Portanto, $C \subset B$. 
- Se $b \leq c$, então $B \subseteq \mathcal{X}_{\downarrow}^{b}(f)=\{q \in \mathcal{D}: f(q)<b \leq c\} \subseteq \mathcal{X}_{\downarrow}^{c}(f)$. Logo, como $C$ é uma CC, então existem caminhos em $C$ para todos os pixels de $B$, pois $B \cap C \neq \emptyset$. Portanto, $B \subseteq C$.

Assim concluímos que o poset $(\mathcal{L}(f), \subseteq)$ é uma árvore. A prova para o poset $(\mathcal{U}(f), \subseteq)$ segue de forma análoga.

Por meio dos argumentos da prova da Proposição 4.12 pode-se derivar o Corolário 4.13.

Corolário 4.13. Se $A, B \in \mathcal{L}(f)$ (respectivamente, $A, B \in \mathcal{U}(f)$ ) então ou $A \subseteq B$, ou $B \subseteq A$, ou $A \cap B=\emptyset$.

Na Figura 4.7 são apresentadas as árvores de componentes construídas com os conjuntos de níveis apresentados na Figura 4.6.
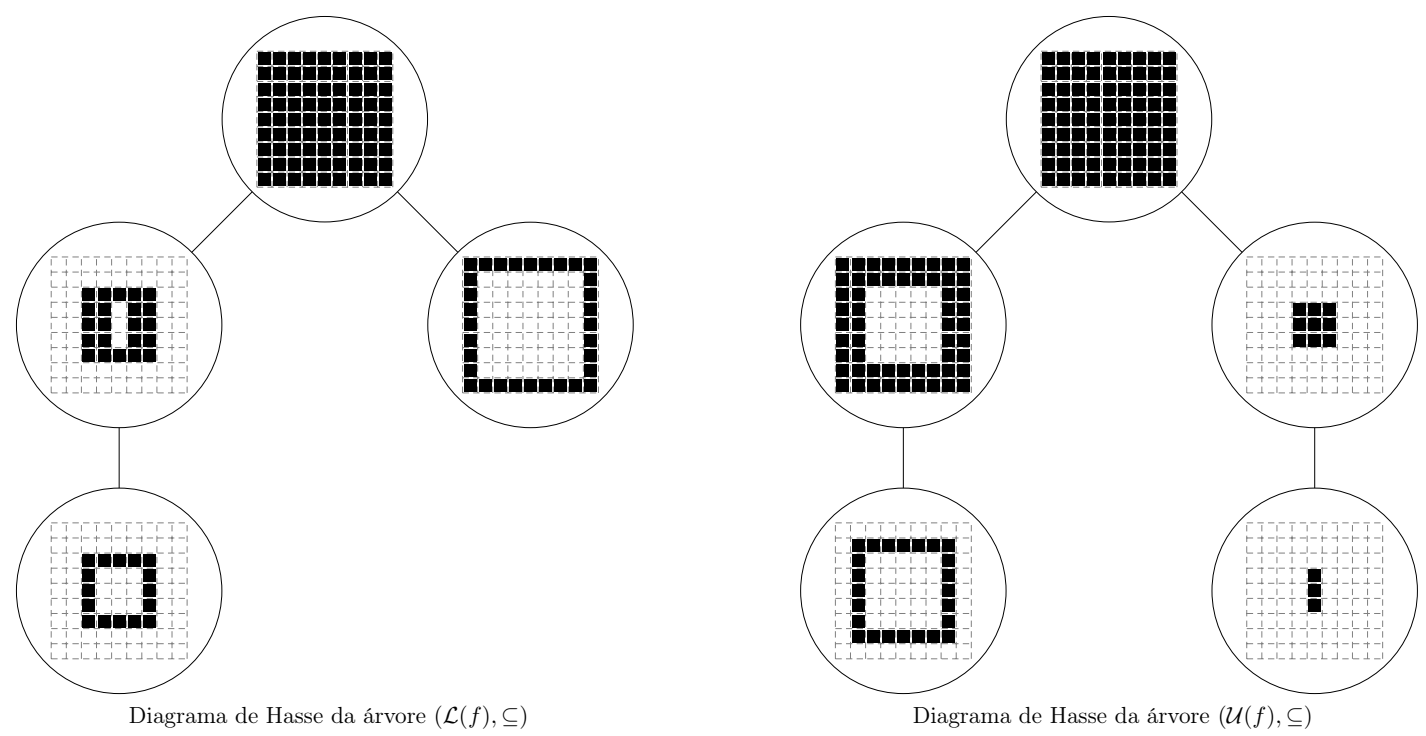

Figura 4.7: Exemplos de árvores de componentes.

A árvore de componentes dos conjuntos de níveis inferiores (respectivamente, superiores) é uma representação completa de uma imagem que pode ser representada por uma estrutura de dados compacta e não redundante conhecida por min-tree (respectivamente, max-tree) (Salembier et al. , 1998). Nesta estrutura, um pixel $p \in \mathcal{D}$ é associado ao menor CC que o contém e pelo uso da relação de parentesco entre os vértices da árvore, ele também está associado a todos os outros CCs ancestrais na árvore.

Definição 4.14 (Menor componente). Chama-se de menor componente um vértice $A$ contendo um pixel $p \in \mathcal{D}$ de uma árvore $\mathcal{T}$ tal que não exista descendentes de $A$ em $\mathcal{T}$ contendo $p$. Assim, é denotado por $\mathcal{S C}(\mathcal{T}, p)$ o menor $\mathrm{CC}$ contendo $p$ na árvore $\mathcal{T}$.

Na Figura 4.8 são apresentadas as árvores da Figura 4.7 destacando em vermelho os pixels que estão posicionados nos seus respectivos menores componentes.

\section{Árvore de componentes estendida}

Neste trabalho também é considerado as versões estendidas das árvores de componentes, ou seja, as árvores contendo CCs extraídos de todos os conjuntos de níveis de uma imagem (Jones 

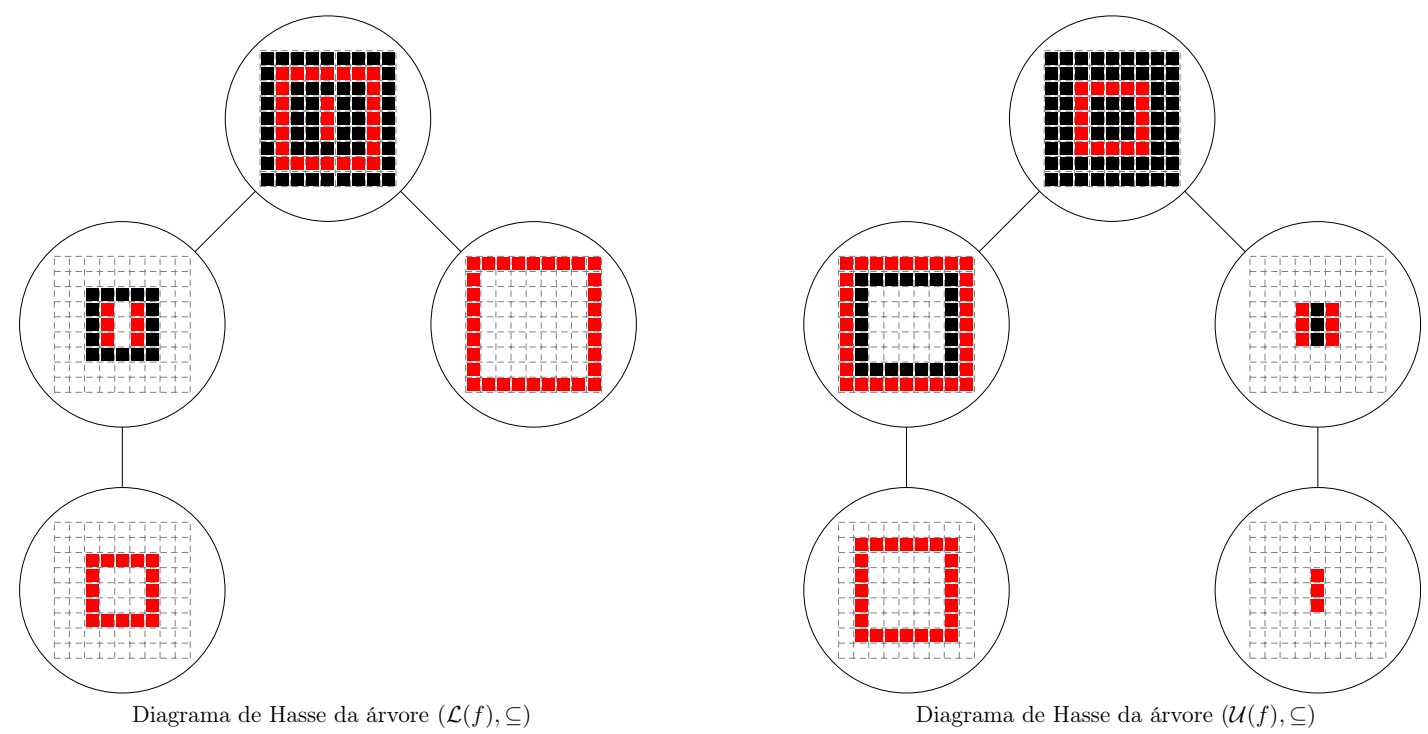

Figura 4.8: Exemplos de árvores de componentes nos quais são destacados em vermelho a associação entre os pixels e os menores CCs.

, 1999). Assim, considere

$$
\operatorname{Ext}(\mathcal{L}(f))=\left\{(C, \lambda) \in \mathcal{L}(f) \times \mathbb{K}: C \in \mathcal{C C}\left(\mathcal{X}_{\downarrow}^{\lambda}(f)\right)\right\}
$$

$\mathrm{e}$

$$
\operatorname{Ext}(\mathcal{U}(f))=\left\{(C, \lambda) \in \mathcal{U}(f) \times \mathbb{K}: C \in \mathcal{C C}\left(\mathcal{X}_{\lambda}^{\uparrow}(f)\right)\right\}
$$

os conjuntos dos pares CCs e limiares extraídos de todos os conjuntos de níveis inferiores e superiores, respectivamente. Embora, também pode-se construir similarmente $\operatorname{Ext}(\mathcal{S A T}(f))$, neste trabalho, são utilizados somente as versões estendidas das árvores componentes, max-tree e min-tree.

Em adição, considere uma relação denotada por $\sqsubseteq$, sobre o conjunto $\operatorname{Ext}(\mathcal{L}(f))$, tal que dois elementos $(A, i),(B, j) \in \operatorname{Ext}(\mathcal{L}(f))$ estão relacionados por $\sqsubseteq$ e escritos como $(A, i) \sqsubseteq(B, j)$ se, e somente se, $A, B \subseteq \mathcal{D}, i, j \in \mathbb{K}, A \subseteq B$ e $i \leq j$. Analogamente, define-se esta relação também sobre o conjunto $\operatorname{Ext}(\mathcal{U}(f))$, ou seja, $(A, i),(B, j) \in \operatorname{Ext}(\mathcal{U}(f))$ se, e somente se, $A, B \subseteq \mathcal{D}, i, j \in \mathbb{K}$, $A \subseteq B$ e $i \geq j$. Portanto, os posets $(\operatorname{Ext}(\mathcal{L}(f)), \sqsubseteq),(\operatorname{Ext}(\mathcal{U}(f)), \sqsubseteq)$ e $(\operatorname{Ext}(\mathcal{S} \mathcal{A} \mathcal{T}(f)), \sqsubseteq)$ são as versões estendidas das árvores $(\mathcal{L}(f), \subseteq),(\mathcal{U}(f), \subseteq)$ e $(\mathcal{S} \mathcal{A} \mathcal{T}(f), \subseteq)$, respectivamente.

Observe que, se $\mathcal{T}$ é uma árvore (max-tree ou min-tree) construída a partir de uma imagem $f \in \mathcal{D}$, então o menor CC contendo um pixel $p \in \mathcal{D}$ é o vértice $\mathcal{S C}(\mathcal{T}, p)$ e na versão estendida de $\mathcal{T}$ é o vértice $(\mathcal{S C}(\mathcal{T}, p), f(p))$. Na Figura 4.9 são apresentadas as versões estendidas das árvores da Figura 4.7 destacando em vermelho os pixels que estão posicionados nos seus respectivos menores componentes.

\section{Algoritmo para construção de árvore de componentes}

Diversos algoritmos foram propostos para criar uma árvore de componentes de uma imagem, os quais podem ser classificados em duas categorias: (1) algoritmos com base em inundação (em inglês, flooding), como proposto por Nistér e Stewénius (2008); Salembier et al. (1998) e Wilkinson et al. (2011). Nesses algoritmos, a construção da árvore inicia-se partindo da raiz para as folhas por meio de um processo de crescimento de região e propagação de nível de cinza para zonas planas 

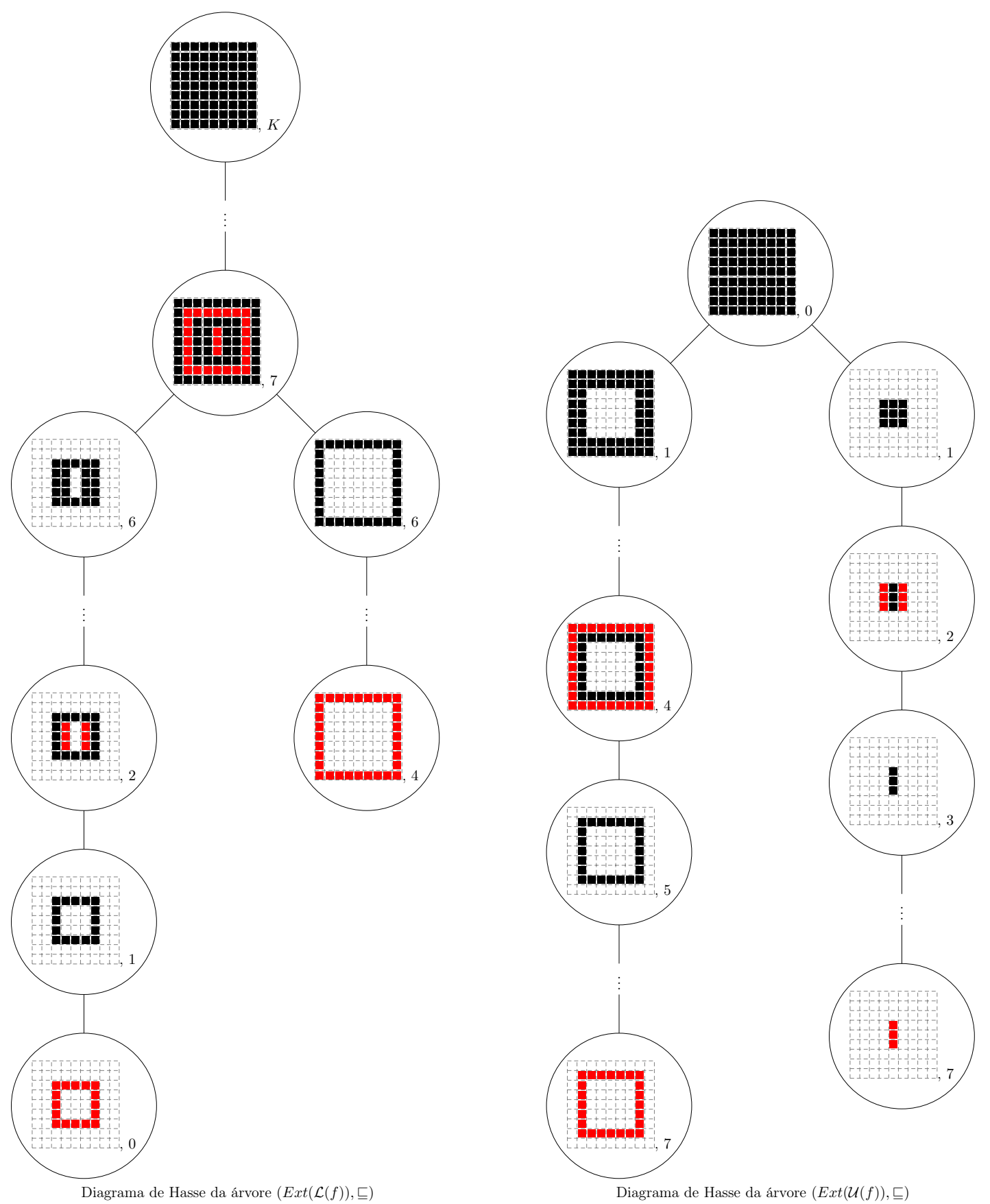

Figura 4.9: Exemplos de construções de árvores de componentes estendidas.

adjacentes até atingir as extremas (máximas para max-tree e mínimas para min-tree) regionais e (2) algoritmos com base em imersão (em inglês, immersion), como proposto por Najman e Couprie (2006) e Berger et al. (2007). Nesses algoritmos, a computação inicia ordenando os pixels das zonas planas da imagem pelo seu nível de cinza. Em seguida, os pixels vão sendo processado de forma ordenada em que cada pixel inicialmente é considerado um conjunto isolado. Neste processamento ordenado, os conjuntos disjuntos vão sendo fundidos e formando uma árvore por meio do tradicional algoritmo de union-find proposto por Tarjan (1975).

Em Carlinet e Géraud (2014) foi realizado um amplo estudo sobre os principais algoritmos para construção da árvore de componentes, onde eles apresentam uma árvore de decisão, mostrada na Figura 4.10, enfatizando os melhores algoritmos com base em critérios de limitação de memória, 
profundidade da imagem e tempo de execução. Percebe-se que quase todos os caminhos da árvore de decisão proposta por Carlinet e Géraud (2014) levam ao algoritmo de Berger et al. (2007) e suas otimizações por union-find (Tarjan, 1975).

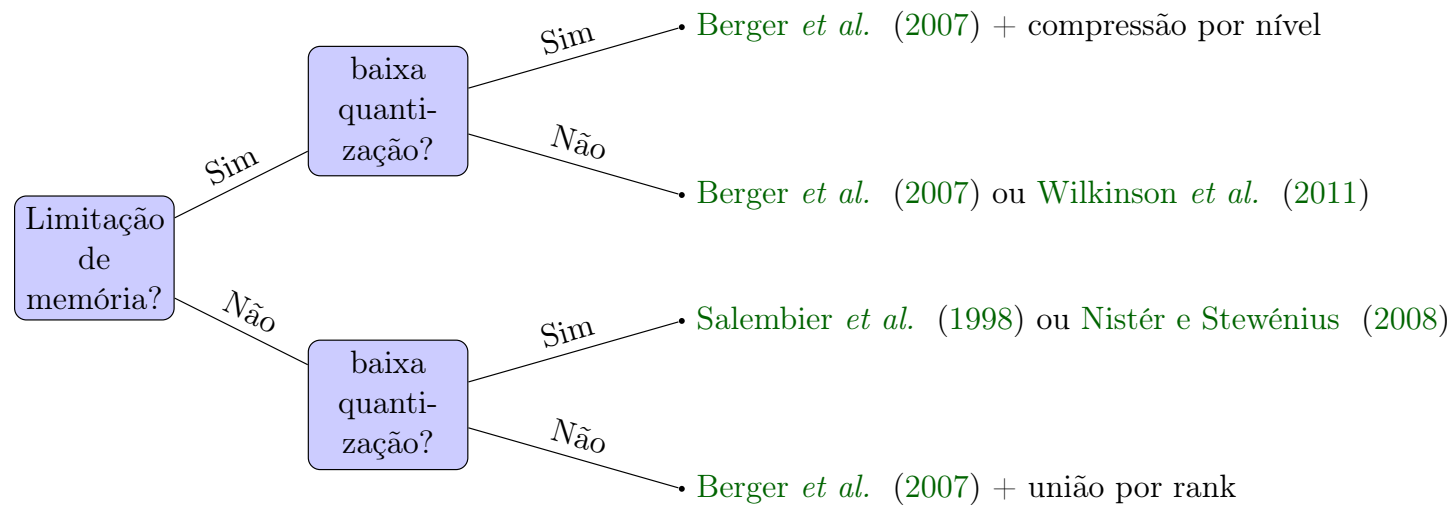

Figura 4.10: Árvore de decisão para auxiliar a escolha de um algoritmo para obter árvore de componentes para uma determinada aplicação.

Os algoritmos baseados em union-find, consistem em rastrear CCs disjuntos e fundi-los por meio de um processamento bottom-up. Primeiramente, os pixels das zonas planas da imagem são ordenados pelo nível de cinza em uma função $f_{\text {sort }}: I_{\text {sort }} \subset \mathbb{N} \rightarrow \mathcal{D}$ tal que $\forall i, j \in I_{\text {sort }}, i<j \Rightarrow$ $f\left(f_{\text {sort }}(i)\right) \leq f\left(f_{\text {sort }}(j)\right)$. Então, cada pixel $p \in \mathcal{D}$ é representado por um conjunto contendo um único elemento (função make-set), ou seja, $\{p\}$. Em seguida, os pixels são processados em ordem decrescente da função $f_{\text {sort }}$, isto é, na ordem $\left(f_{\text {sort }}\left(\left|I_{\text {sort }}\right|\right), f_{\text {sort }}\left(\left|I_{\text {sort }}\right|-1\right), \ldots, f_{\text {sort }}(0)\right)$ que significa o processamento partindo das folhas para raiz da árvore. Quando um pixel $p \in \mathcal{D}$ é processado, é procurado (função find-root) por representantes $q \in \mathcal{D}$ de conjuntos disjuntos adjacentes já processados e se funde (função merge-set) formando um novo conjunto representado por $p$. Este processo de fusão consiste em atualizar os representantes de $p$ e $q$ em uma função chamada parent de tal forma que $p$ seja o pai dos representantes fundidos com $p$. Assim, o algoritmo de union-find baseia-se em três funções: $(i)$ make-set(parent, $x$ ) que constrói um conjunto com um único elemento $\{x\}$; (ii) find-root(parent, $x)$ que localiza o representante do conjunto contendo $x$; (iii) merge-set (parent, $x, y)$ que funde os conjuntos representados por $x$ e $y$ e define $x$ como o representante do novo conjunto. Com base nessas funções, o Algoritmo 4.1 realiza a construção genérica da relação de parentesco entre os CCs de uma árvore de componentes.

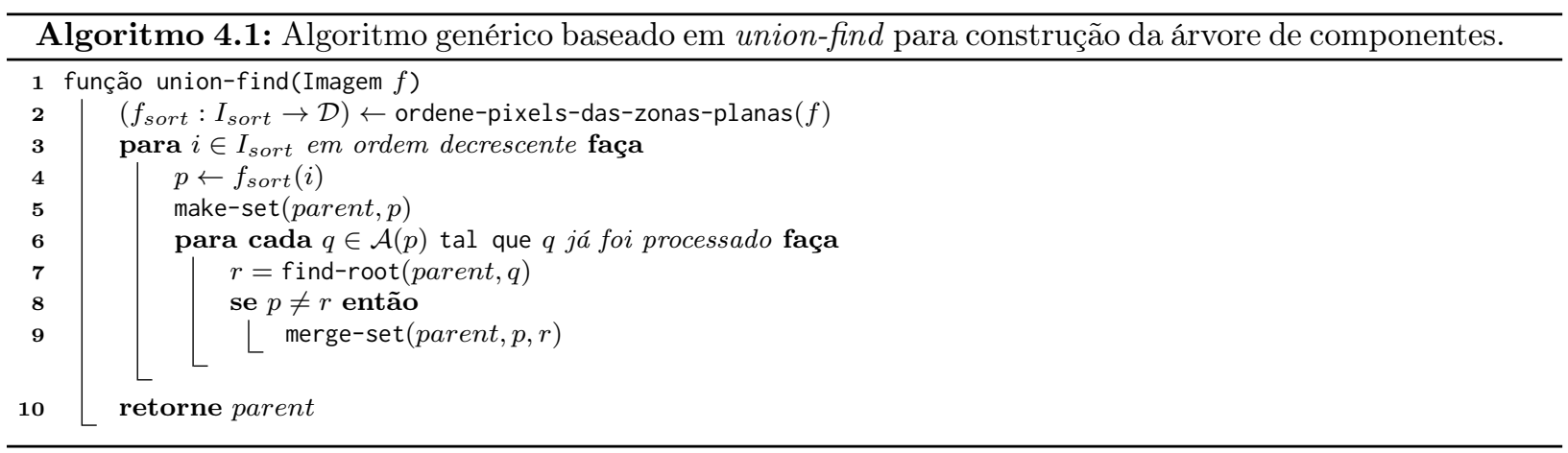

Este algoritmo codifica uma árvore de componentes $\mathcal{T}$ usando uma função (ou computacionalmente vetor) parent $: \mathcal{D} \rightarrow \mathcal{D}$ que armazena a relação de parentesco que existe entre os CCs de $\mathcal{T}$. 
Neste caso, a função parent representa cada CC por um pixel chamado por elemento canônico, cujo seu nível de cinza é o mesmo usado para obter o menor conjunto de nível que contém o CC. Assim, seja $p_{r} \in \mathcal{D}$ o pixel canônico que representa o CC da raiz da árvore $\mathcal{T}$, e então, um pixel $p \in \mathcal{D}$ é canônico se, e somente se, $p=p_{r}$ ou se $\mathcal{T}$ é a max-tree então $f(\operatorname{parent}(p))<f(p)$, ou se $\mathcal{T}$ é a min-tree então $f(\operatorname{parent}(p))>f(p)$. Além disso, como mostrado por Berger et al. (2007), a função parent após um processo de canonização (isto é, fazer com que os pais dos elemento não-canônicos sejam os representantes dos CCs) satisfaz a seguinte propriedade: se $p \in \mathcal{D}$, então parent( $p)$ é canônico. Assim, se $p$ é um pixel canônico, então o pai do menor CC contendo $p \in \mathcal{D}$ é dado por $\mathcal{S C}(\mathcal{T}$, parent $(p))$, caso contrário, é $\mathcal{S C}(\mathcal{T}$, parent $($ parent $(p)))$, isto é

$$
p \in \mathcal{D}, \operatorname{Pai}(\mathcal{S C}(\mathcal{T}, p))= \begin{cases}\mathcal{S C}(\mathcal{T}, \text { parent }(p)), & \text { se } p \text { é um elemento canônico, } \\ \mathcal{S C}(\mathcal{T}, \operatorname{parent}(\operatorname{parent}(p))), & \text { caso contrário. }\end{cases}
$$

Note que, se $p \in \mathcal{D}$ é canônico, então $\mathcal{S C}(\mathcal{T}, p)$ é o mesmo CC para todos os outros pixels $q \in \mathcal{D}$ não-canônico que tem como parent o pixel $p$, isto é, $\mathcal{S C}(\mathcal{T}, p)=\mathcal{S C}(\mathcal{T}, q)$ tal que parent $(q)=p$. Com isso, pode-se construir uma estrutura de dados mais simples, mas ainda assim compacta e não redundante, para manipular a árvore de componentes. Na Figura 4.11 é apresentado um exemplo de computação para árvores de componentes (min-tree) apresentada na Figura 4.7, onde é fácil perceber estas propriedades sobre a função parent.

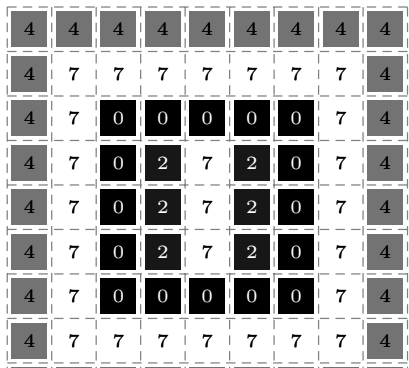

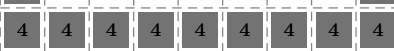

Imagem de entrada $f$

\begin{tabular}{|l|l|l|l|l|l|l|l|l|}
\hline 2 & 2 & 2 & 2 & 2 & 2 & 2 & 2 & 2 \\
\hline 2 & 0 & 0 & 0 & 0 & 0 & 0 & 0 & 2 \\
\hline 2 & 0 & 5 & 5 & 5 & 5 & 5 & 0 & 2 \\
\hline 2 & 0 & 5 & 4 & 1 & 3 & 5 & 0 & 2 \\
\hline 2 & 0 & 5 & 4 & 1 & 3 & 5 & 0 & 2 \\
\hline 2 & 0 & 5 & 4 & 1 & 3 & 5 & 0 & 2 \\
\hline 2 & 0 & 5 & 5 & 5 & 5 & 5 & 0 & 2 \\
\hline 2 & 0 & 0 & 0 & 0 & 0 & 0 & 0 & 2 \\
\hline 2 & 2 & 2 & 2 & 2 & 2 & 2 & 2 & 2 \\
\hline
\end{tabular}

Zonas planas ordenadas

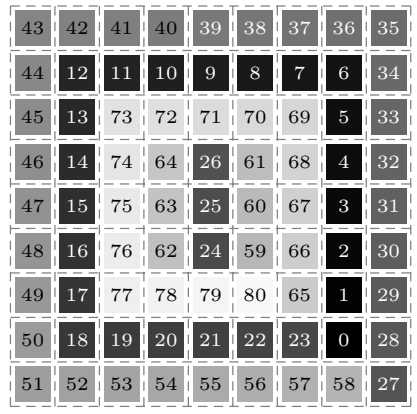

Pixels das zonas planas ordenados

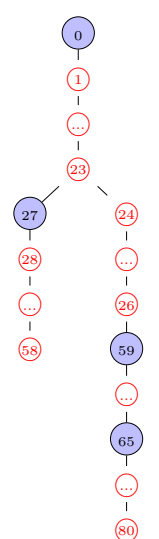

Relação de parentesco da função parent

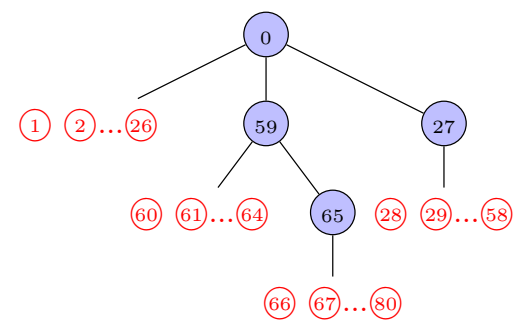

Relação de parentesco da função parent canonizada

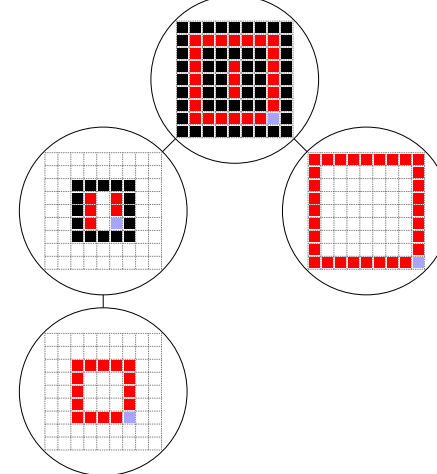

Diagrama de Hasse $(\mathcal{L}(f), \subseteq)$

Figura 4.11: Exemplo de computação da min-tree. Note que, os pixels em azul são canônicos e em vermelho os não-canônicos.

Os algoritmos baseados em union-find requerem que os pixels das zonas planas da imagem estejam ordenados pelos níveis de cinza (ordem crescente para min-tree e ordem decrescente para a 
max-tree). Para esta ordenação, é proposto neste trabalho o Algoritmo 4.2 que utiliza uma fila de prioridade proposta por Falcão et al. (2000) no qual suas operações de consulta, inserção e remoção são realizadas em tempo constante. A ideia central do algoritmo de ordenação é inserir todos os pixels na fila de prioridade com política LIFO (último a entrar, primeiro a sair) e depois, consumir os pixels da fila de prioridade na ordem de prioridade. Para cada pixel consumido são retirados os pixels adjacentes na zona plana do pixel em questão e inseri-los novamente na fila de prioridade para que os mesmos sejam os próximos elementos a serem consumidos. Assim, uma zona plana é processada por vez. Este algoritmo analisa cada pixel exatamente uma única vez e para cada pixel analisado, é visitado seus vizinhos. Logo, a complexidade do algoritmos é $\mathcal{O}(|\mathcal{D}| \times|\mathcal{A}|)$, onde $|\mathcal{D}|$ denota a cardinalidade de $\mathcal{D}$ e $|\mathcal{A}|$ a quantidade máxima de pixel na vizinhança $\mathcal{A}$.

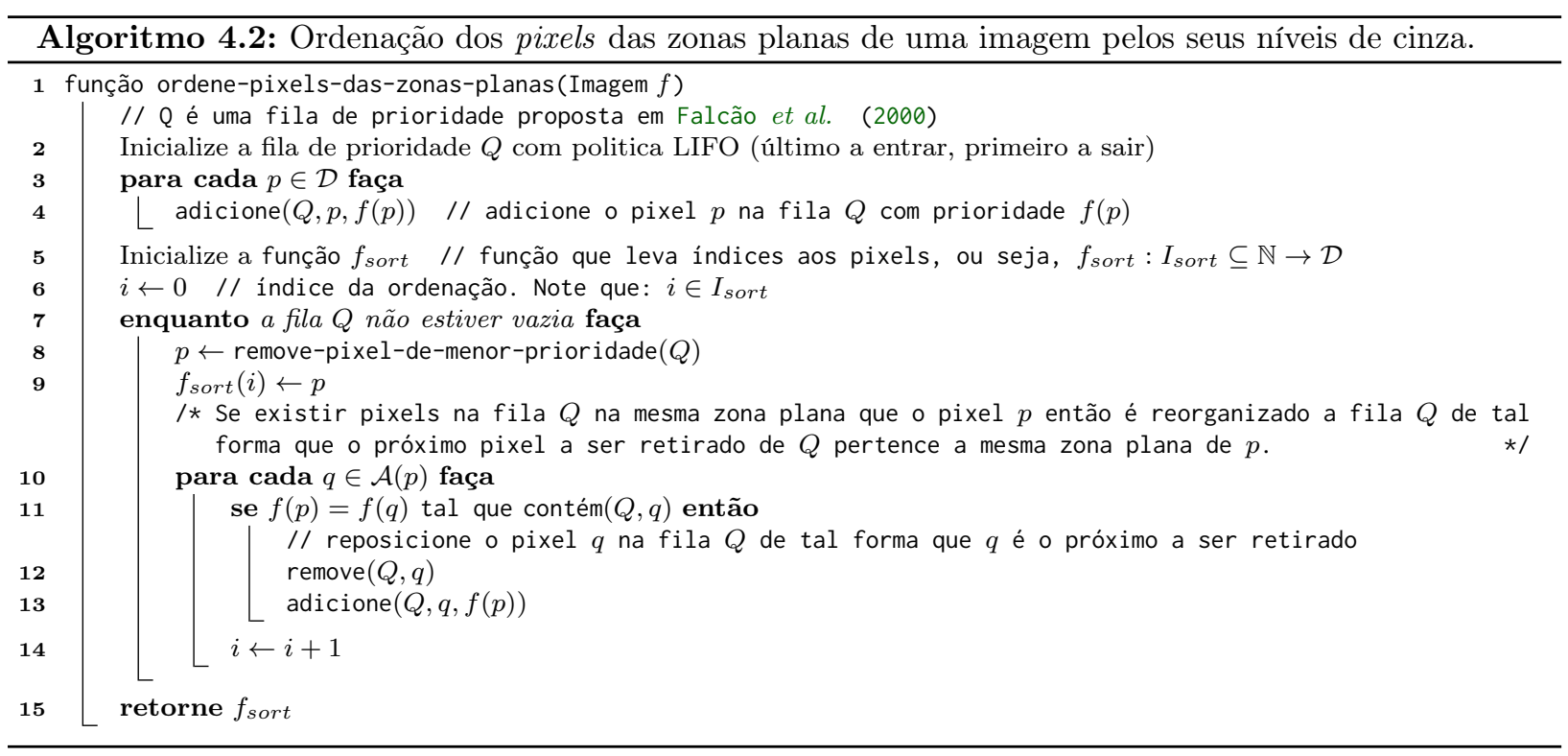

O Algoritmo 4.1 tem complexidade de espaço $\mathcal{O}(|\mathcal{D}|)$ e tempo $\mathcal{O}\left(|\mathcal{D}|^{2}\right)$, pois a função find-root, tem complexidade $\mathcal{O}(|\mathcal{D}|)$ e cada pixel $p$ é processado uma única vez. Em, Tarjan (1975) são discutidas duas otimizações para evitar a complexidade de tempo quadrática: compactação de caminho e união por ranque. Se adicionar a otimização de compactação de caminho, a complexidade de espaço é $\mathcal{O}(2|\mathcal{D}|)$ e a complexidade de tempo é $\mathcal{O}(|\mathcal{D}| \times \log (|\mathcal{D}|))$ e, se adicionar também a otimização de união por ranque, a complexidade de espaço é $\mathcal{O}(4|\mathcal{D}|)$ e a complexidade de tempo é $\mathcal{O}(|\mathcal{D}| \times \log (\log (|\mathcal{D}|)))$. Um estudo completo sobre comparações de complexidade computacional e tempo de execução dos algoritmos para construção de árvore de componentes pode ser encontrado em Carlinet e Géraud (2014). Por fim, uma implementação completa para construção da árvore de componentes usando o algoritmo de Berger et al. (2007) é apresentada no Algoritmo 4.3.

\subsection{2 Árvore de formas}

A árvore de formas, proposta por Monasse e Guichard (2000), é uma importante estrutura morfológica que representa uma imagem de uma maneira auto-dual. Ela pode ser vista como o resultado da fusão das árvores de componentes, min-tree e max-tree, em uma única árvore. Diversos trabalhos encontrados na literatura utilizam árvores de formas, por exemplo, em problemas de filtragem (Caselles e Monasse, 2002; Keshet, 2007), segmentação (Cardelino et al., 2006; Desolneux et al., 2001; Xu et al., 2012), extração de resíduos (Alves e Hashimoto, 2014; Alves et al. , 2013), registro (Monasse, 2000), entre outros. 


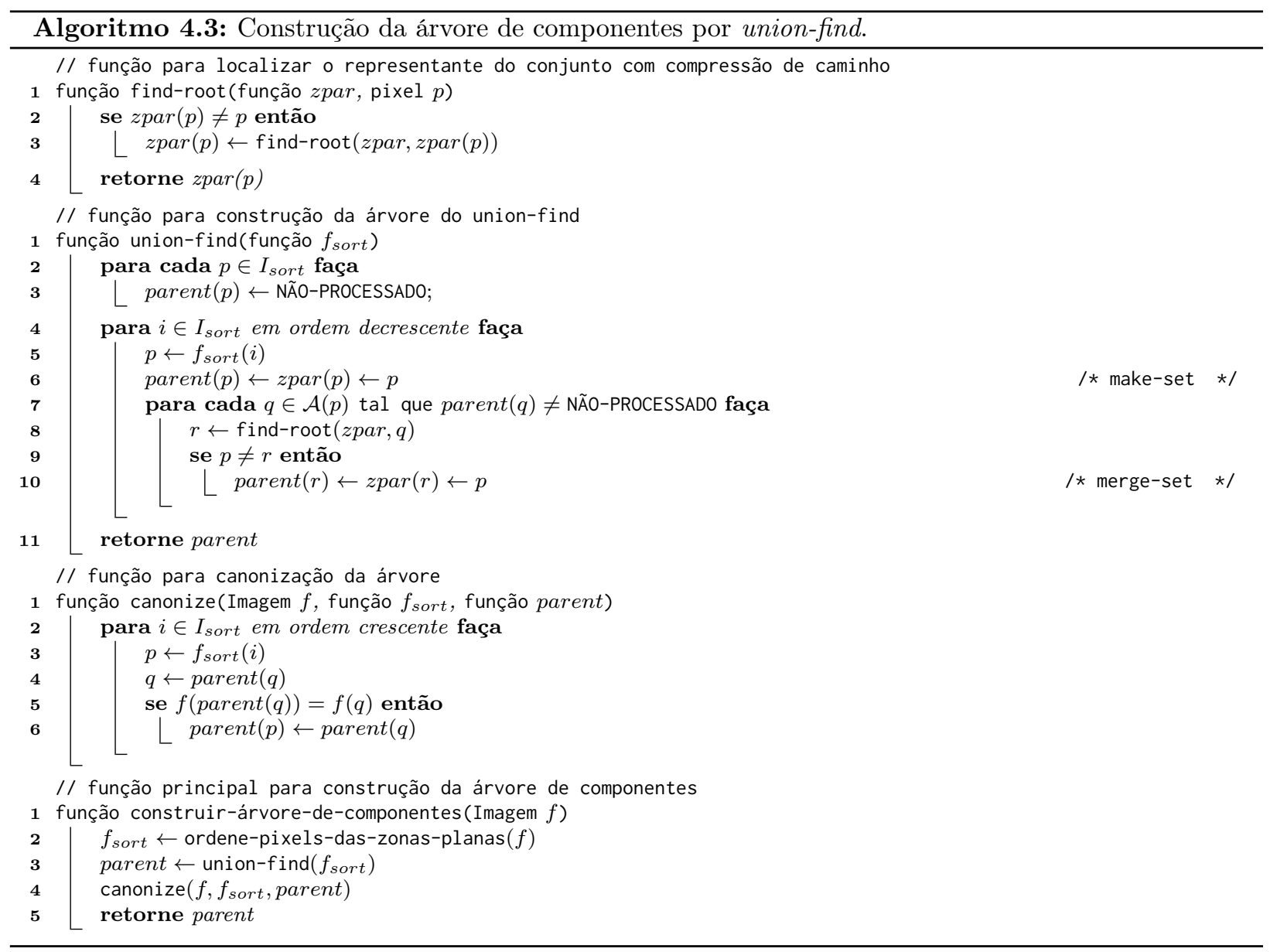

Essa árvore pode ser vista em uma representação hierárquica dos CCs dos conjuntos de níveis inferiores e superiores sem buracos (ou melhor, com os buracos preenchidos). A fusão entre estas duas árvores ocorre pela noção de buracos internos dos CCs de $\mathcal{L}(f)$ e $\mathcal{U}(f)$, e isso pode ser visualizado na Figura 4.7, na qual pode-se perceber que os buracos dos CCs da max-tree podem ser preenchidos por CCs da min-tree. Da mesma forma, os buracos dos CCs da min-tree podem ser preenchidos pelos CCs da max-tree. A fusão destas duas árvores nos permite unificar, em uma única árvore, objetos presentes na max-tree e na min-tree, conforme pode ser observado na Figura 4.12.

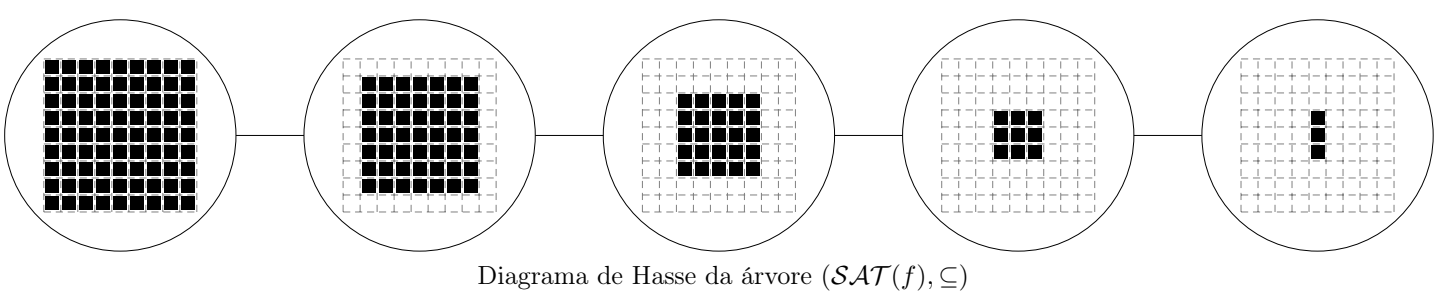

Figura 4.12: Um exemplo de árvore de formas da imagem da Figura 4.6.

Mas esta hierarquia só é possível, se consideramos conectividades complementares para os CCs dos conjuntos de níveis inferiores e superiores, como por exemplo, 4-conectividade para os CCs dos conjuntos de níveis inferiores, isto é, $\mathcal{L}(f)=\left\{C: C \in \mathcal{C C}\left(\mathcal{X}_{\downarrow}^{\lambda}(f), \mathcal{A}_{4 c}\right), \lambda \in \mathbb{K}\right\}$, e 8-conectividade para os CCs dos conjuntos de níveis superiores, ou seja, $\mathcal{U}(f)=\left\{C: C \in \mathcal{C C}\left(\mathcal{X}_{\lambda}^{\uparrow}(f), \mathcal{A}_{8 c}\right), \lambda \in \mathbb{K}\right\}$. Assim, evita-se possíveis problemas topológicos como mencionado por Caselles e Monasse (2010); Monasse (2000); Monasse e Guichard (2000); Najman e Géraud (2013) e Géraud et al. (2013). 
Para definir a árvore de formas, primeiramente, considere sat $: \mathcal{P}(\mathcal{D}) \rightarrow \mathcal{P}(\mathcal{D})$ um operador de preenchimento de buracos internos de um CC (Caselles et al., 2008).

Definição 4.15 (Buracos). Denomina-se de buracos internos de $A \in \mathcal{L}(f) \cup \mathcal{U}(f)$, denotado por Int $(A)$, os CCs de $\mathcal{D} \backslash A$ incluídas em sat $(A)$. Também, denomina-se de exterior de $A$, denotado por $\operatorname{Ext}(A)$, o conjunto $\mathcal{D} \backslash \operatorname{sat}(A)$. Note que

$$
\operatorname{sat}(A)=A \cup \bigcup_{H \in \operatorname{Int}(A)} H
$$

onde as uniões são disjuntas. Na Figura 4.13 é apresentado um exemplo de um CC $A$ com seu conjunto $\operatorname{sat}(A)$ e $\operatorname{Ext}(A)$.

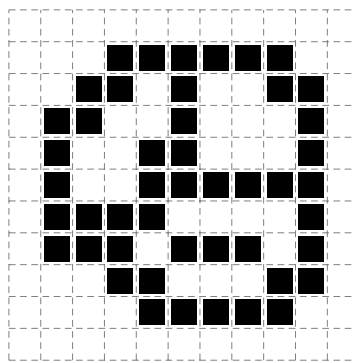

Conjunto $\mathcal{X} \subseteq \mathcal{D}$

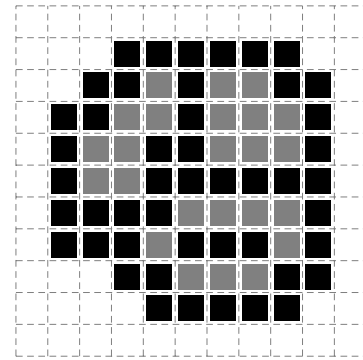

$\operatorname{sat}(A)$

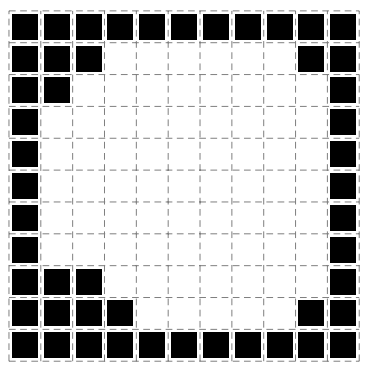

$\operatorname{Ext}(A)$

Figura 4.13: Exemplo mostrando um $C C A$ com seu conjunto sat $(A)$ e $\operatorname{Ext}(A)$.

Observe que, o complemento de um CC $A$ é $\operatorname{Ext}(A) \cup \operatorname{Int}(A)$ e se $H \in \operatorname{Int}(A)$ então $\operatorname{sat}(H) \subseteq$ $\operatorname{sat}(A)$. Além do mais, se $A$ é um $\mathrm{CC} \operatorname{de} \mathcal{U}(f)$ com buracos internos, então $\operatorname{Int}(A)$ são CCs de $\mathcal{L}(f)$, pois $\operatorname{Int}(A)=(\mathcal{D} \backslash A) \cap \operatorname{sat}(A) \subseteq \mathcal{D} \backslash \mathcal{X}_{\downarrow}^{\lambda}=\mathcal{X}_{\lambda}^{\uparrow}$. Agora, sejam $\mathcal{S} \mathcal{A} \mathcal{T}_{\mathcal{L}}(f)=\{\operatorname{sat}(C): C \in \mathcal{L}(f)\}$ e $\mathcal{S A T}_{\mathcal{U}}(f)=\{\operatorname{sat}(C): C \in \mathcal{U}(f)\}$ as famílias de CCs dos conjuntos de níveis inferiores e superiores sem buracos, respectivamente, e considere $\mathcal{S} \mathcal{A} \mathcal{T}(f)=\mathcal{S} \mathcal{A} \mathcal{T}_{\mathcal{L}}(f) \cup \mathcal{S} \mathcal{A} \mathcal{T}_{\mathcal{U}}(f)$. Os elementos de $\mathcal{S} \mathcal{A} \mathcal{T}(f)$ são denominados de formas. Para mostrarmos que o poset $(\mathcal{S} \mathcal{A} \mathcal{T}(f), \subseteq)$ é uma árvore precisa-se ter uma garantia que quaisquer duas formas de $\mathcal{S} \mathcal{A} \mathcal{T}(f)$ são disjuntas ou comparáveis. Neste sentido, o Teorema 4.16 proposto por Monasse (2000) garante este fato. Portanto, o par $(\mathcal{S A T}(f), \subseteq$ ), é uma árvore conhecida por árvore de formas (Caselles e Monasse, 2010; Caselles et al., 2008; Géraud et al., 2013; Monasse e Guichard, 2000).

Teorema 4.16 (Caselles e Monasse (2010)). Se $A, B \in \mathcal{S A T}(f)$. Então, ou $A \subseteq B$, ou $B \subseteq A$, ou $A \cap B=\emptyset$.

Prova: A prova deste teorema pode ser encontrada em Caselles e Monasse (2010) na Pag. 19, Teorema 2.16.

Proposição 4.17. O poset $(\mathcal{S} \mathcal{A} \mathcal{T}(f), \subseteq)$ é uma árvore.

Prova: Para mostrar que $(\mathcal{S A T}(f), \subseteq)$ é uma árvore basta verificar se a Definição 4.1 é satisfeita.

1. Seja $\alpha$ o menor nível de cinza de $f$. Por definição, $\mathcal{X}_{\alpha}^{\uparrow}(f)=\{x \in \mathcal{D}: f(x) \geq \alpha\}$. Logo, todos os pixels $x \in \mathcal{D}$ satisfazem $f(x) \geq \alpha$, pois $\alpha$ é o menor nível de cinza de $f$. Como $\mathcal{D}$ é um subconjunto retangular e compacto de $\mathbb{Z}^{2}$ então $\operatorname{sat}(\mathcal{D})=\mathcal{D}$ e assim temos que $\operatorname{sat}(\mathcal{D}) \in \mathcal{S} \mathcal{A} \mathcal{T}(f)$. Portanto, $\forall A \in \mathcal{S} \mathcal{A} \mathcal{T}(f)$ temos que $A \subseteq \operatorname{sat}(\mathcal{D})$. 
2. Sejam $A, B, C \in \mathcal{S} \mathcal{A} \mathcal{T}(f)$ tais que $A \subseteq B$ e $A \subseteq C$. Então, como $B \cap C \neq \emptyset$ segue, pelo Teorema 4.16, que ou $B \subseteq C$, ou $C \subseteq B$.

Assim, concluímos que $(\mathcal{S A T}(f), \subseteq)$ é uma árvore.

A árvore de formas $\mathcal{T}=(\mathcal{S} \mathcal{A} \mathcal{T}(f), \subseteq)$ pode ser armazenada de forma compacta e sem redundância. Desta forma, um pixel $p$ é associado somente a menor forma $\mathcal{S C}(\mathcal{T}, p)$ que contém $p$ e pela relação de parentesco da árvore, este pixel está associado também a todas as formas ancestrais.

\section{Algoritmo para construção de árvore de formas}

O primeiro algoritmo conhecido para construir uma árvore de formas, o Fast Line Level Transform (FLLT), proposto por Monasse e Guichard (2000), onde a árvore de formas é construída por meio de uma fusão das árvores min-tree e max-tree. Neste algoritmo precisa-se conhecer um pixel de cada buraco dos CCs dos conjuntos de níveis e isso pode ser computado localmente e eficientemente, em imagens 2D, enquanto se constroem as árvores de componentes, min-tree e max-tree, por meio da característica de Euler ${ }^{1}$. Um segundo algoritmo, o Fast Level Set Transform (FLST), também foi proposto por Monasse (2000) e é baseado na abordagem de crescimento de regiões. Este algoritmo extrai cada ramo da árvore partindo das folhas até a raiz, mas este processo faz com que muitos pixels sejam revisitados e por isso sua complexidade no pior caso é $\mathcal{O}\left(|\mathcal{D}|^{2}\right)$. Recentemente, Géraud et al. (2013) e Najman e Géraud (2013) mostram que o algoritmo de Berger et al. (2007) para construir uma árvore de componentes é surpreendentemente capaz de construir uma árvore de formas. Basicamente, o algoritmo de Berger et al. (2007) é composto por três etapas: (i) ordenação dos pixels; (ii) computação da union-find; (iii) canonização da árvore. Então, Géraud et al. (2013) propôs uma nova ordenação dos pixels e aplicou os passos (ii) e (iii) do algoritmo de Berger et al. (2007) como apresentado no Algoritmo 4.5. Por exemplo, considere na Figura 4.14, a imagem de entrada, os conjuntos de níveis, a árvore de formas e a nova representação da imagem proposta por Géraud et al. (2013) e Najman e Géraud (2013). Note que, para manter a propriedade da

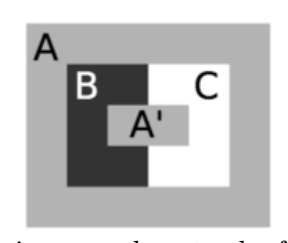

imagem de entrada $f$

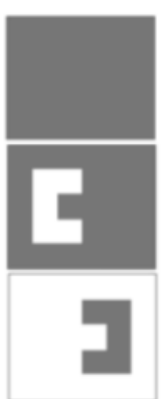

$\mathcal{U}(f)$

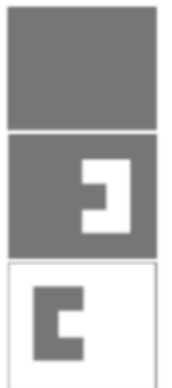

$\mathcal{L}(f)$

Conjuntos de níveis

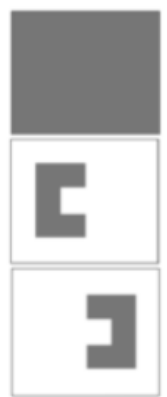

$\mathcal{S} \mathcal{A} \mathcal{T}(f) \quad(\mathcal{S} \mathcal{A} \mathcal{T}(f), \subseteq)$

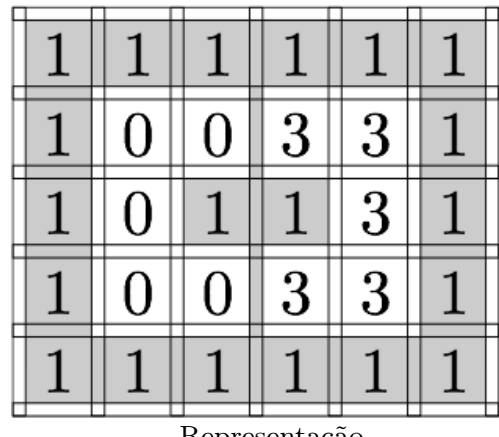

Representação

Figura 4.14: A Figura adaptada de Géraud et al. (2013). Ela apresenta um exemplo contendo a imagem de entrada, os conjuntos de níveis, a árvore de formas e a representação da imagem, respectivamente, da esquerda para direita.

ordenação em uma computação de um CC saturado usando um algoritmo baseado em crescimento de região), é preciso ter caminhos para alcançar as regiões $A$ e $A^{\prime}$ para assim ordená-las antes das

\footnotetext{
${ }^{1}$ A característica de Euler de um conjunto $\mathcal{X} \subseteq \mathcal{D}$ é $E=N_{C}-N_{H}$, onde $N_{C}$ é o número de CCs de $X$ e $N_{H}$ é de buracos de $\mathcal{X}$. Logo, se $\mathcal{X}$ é uma CC então $N_{C}=1$ e assim o número de buracos de $\mathcal{X}$ é $N_{H}=1-E$.
} 
regiões $B$ e $C$ na computação da função $f_{\text {sort }}$. Mas isto só é possível se houver condições de passar entre os pixels e ainda, segundo Géraud et al. (2013), é necessário passar entre os pixels com mais de um valor. Em outras palavras, é necessário uma nova representação da imagem tanto em termos espaciais (domínio) quando em termos de valores (contradomínio), e para este objetivo, duas ferramentas matemáticas são essenciais (Géraud et al., 2013; Najman e Géraud, 2013): (1) grade Khalimsky e (2) funções valor-conjunto. A ideia central é (1) usar grade Khalimsky para criar espaços entre os pixels (domínio da imagem) e (2) usar funções valor-conjunto para modelar diversos valores entre os pixels (contradomínio da imagem). A seguir, é apresentada uma breve descrição sobre esses métodos para depois apresentar o algoritmo proposto por Géraud et al. (2013). Mais detalhes sobre estas teorias podem ser encontrados em Najman e Géraud (2013).

\section{Gride Khalimsky}

A partir dos conjuntos $H_{0}^{1}=\{\{a\}: a \in \mathbb{Z}\}$ e $H_{1}^{1}=\{\{a, a+1\}: a \in \mathbb{Z}\}$, pode-se definir $H^{1}=H_{0}^{1} \cup H_{1}^{1}$ e o conjunto $H^{2}=H^{1} \times H^{1}$ como o produto cartesiano de $H^{1}$ por $H^{1}$. Se um elemento $h \subset \mathbb{Z}^{2}$ é o produto cartesiano de $d$ elementos de $H_{1}^{1}$ e $2-d$ elementos de $H_{0}^{1}$ então é dito que $h$ é uma $d$-face, onde $d$ é a dimensão da face $h$. Assim, se $d=0$ então $h \in H_{0}^{1} \times H_{0}^{1}$. Analogamente, $d=1 \Rightarrow h \in\left(H_{0}^{1} \times H_{1}^{1}\right) \cup\left(H_{1}^{1} \times H_{0}^{1}\right)$ e $d=2 \Rightarrow h \in H_{1}^{1} \times H_{1}^{1}$. O conjunto de todas as faces de $H^{2}$ é chamado de espaço $2 D$ cubos complexos e o conjunto de faces de $H^{2}$ dispostas dentro de uma grade é chamado de grade Khalimsky (Géraud et al., 2013; Najman e Géraud, 2013). Na Figura 4.15 é apresentado um subconjunto de faces de $H^{2}$ com dimensões 0,1 e 2 dispostas em uma grade Khalimsky, de tal forma que as dimensões das faces estão representadas por círculos, retângulos e quadrados, respectivamente.

- Considere $\mathbb{Z}=\{-2,-1,0,1,2\}$

- $H_{0}^{1}=\{\{-2\},\{-1\},\{0\},\{1\},\{2\}\}$

- $H_{1}^{1}=\{\{-2,-1\},\{-1,0\},\{0,1\},\{1,2\}\}$

- $H^{1}=H_{0}^{1} \cup H_{1}^{1}$

- $H^{2}=H^{1} \times H^{1}=\{$

$$
\begin{array}{cccc}
H^{2}=H^{1} \times H^{1} & =\{ & & \\
(\{-2\},\{-2\}), & (\{-2\},\{-2,-1\}), & \ldots, & (\{-2\},\{2\}) \\
(\{-1\},\{-2\}), & (\{-1\},\{-2,-1\}), & \cdots, & (\{-1\},\{2\}) \\
\vdots & \vdots & \vdots & \vdots \\
(\{2\},\{-2\}), & (\{2\},\{-1,-1\}), & \cdots, & (\{2\},\{2\})
\end{array}
$$

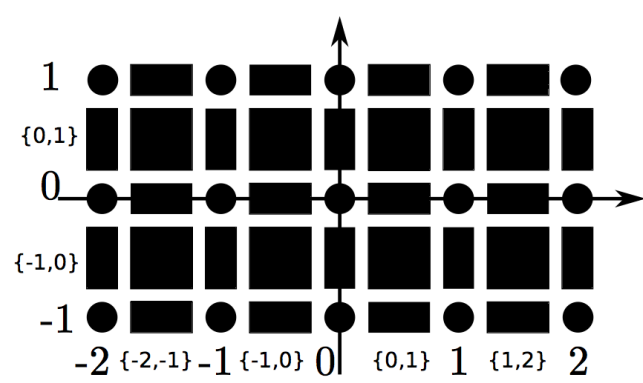

plano cartesiano da grade Khalimsky

Figura 4.15: Figura Adaptada de Najman e Géraud (2013) contendo um plano cartesiano (grade Khalimsky) $H^{2}=H^{1} \times H^{1}$.

Considere os conjuntos $h^{\uparrow}=\left\{h^{\prime} \in H^{2}: h \subseteq h^{\prime}\right\}$ e $h^{\downarrow}=\left\{h^{\prime} \in H^{2}: h^{\prime} \subseteq h\right\}$ e definem-se duas transformações para um subconjunto $E \subseteq H^{2}$, a estrela $s t(E)$ e o fechamento $\operatorname{cl}(E)$, ou seja

$$
s t(E)=\bigcup_{h \in E} h^{\uparrow} \quad \text { e } \quad c l(E)=\bigcup_{h \in E} h^{\downarrow}
$$

Os operadores estrela e fechamento resultam respectivamente, no menor subconjunto de $H_{1}^{2}$ e no menor subconjunto de $H_{0}^{2}$ contendo $E$ (Géraud et al., 2013; Najman e Géraud, 2013). Na Figura 4.16 é apresentado um exemplo de estrela e fechamento de um subconjunto de $E \subseteq H^{2}$. 


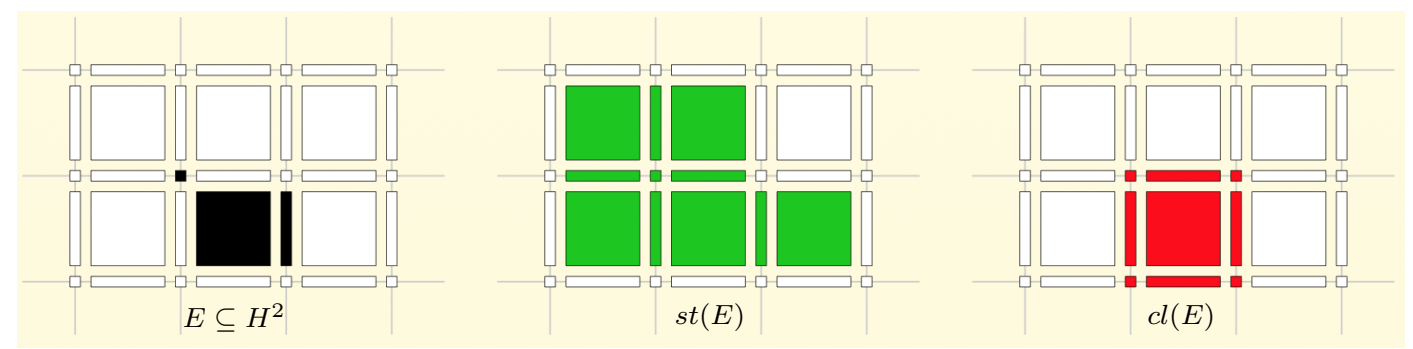

Figura 4.16: Figura adaptada de Géraud et al. (2013) contendo um exemplo de um subconjunto de $E \subset H^{2}$ e respectivamente a estrela e o fechamento de $E$. Note que os elementos de E estão em negrito, os elementos de st $(E)$ estão em verde, e os elementos de $c l(E)$ estão em vermelho.

\section{Função valor-conjunto e interpolação}

Seja $F$ uma função de $X$ em $Y$. Dizemos que $F$ é uma função valor-conjunto, denotada por $F: X \rightsquigarrow Y$, se para todo $x \in X, F$ associa $x$ ao conjunto $F(x) \subseteq Y$ chamado de imagem de $F$ em $x$ (Géraud et al., 2013; Najman e Géraud, 2013). Por meio de uma função $F$, pode-se representar uma imagem $f: \mathcal{D} \subseteq \mathbb{Z}^{2} \rightarrow \mathbb{K} \subseteq \mathbb{Z}$ com espaços maiores, a fim de obter algumas propriedades de continuidade.

Para o espaço do domínio $X$, usa-se subdivisões de $H^{2}$, ou seja, $X=\frac{1}{2} H^{2}=\left\{\frac{1}{2} h: h \in H^{2}\right\}$. Dessa forma, mapeá-se por $m: \mathcal{D} \subseteq \mathbb{Z}^{2} \rightarrow \frac{1}{2} H_{1}^{2}$ cada pixel $p \in \mathcal{D}$ para um elemento de $\frac{1}{2} H_{1}^{2}$ tal que $m(p)=\left\{p_{x}, p_{x}+\frac{1}{2}\right\} \times\left\{p_{y}, p_{y}+\frac{1}{2}\right\}$. Note que assim um pixel $p \in \mathcal{D}$ é uma 2 -face de $X$. Na Figura 4.17 são apresentados os planos cartesianos dos conjuntos $\mathbb{Z}^{2}, H^{2}$ e $\frac{1}{2} H^{2}$.
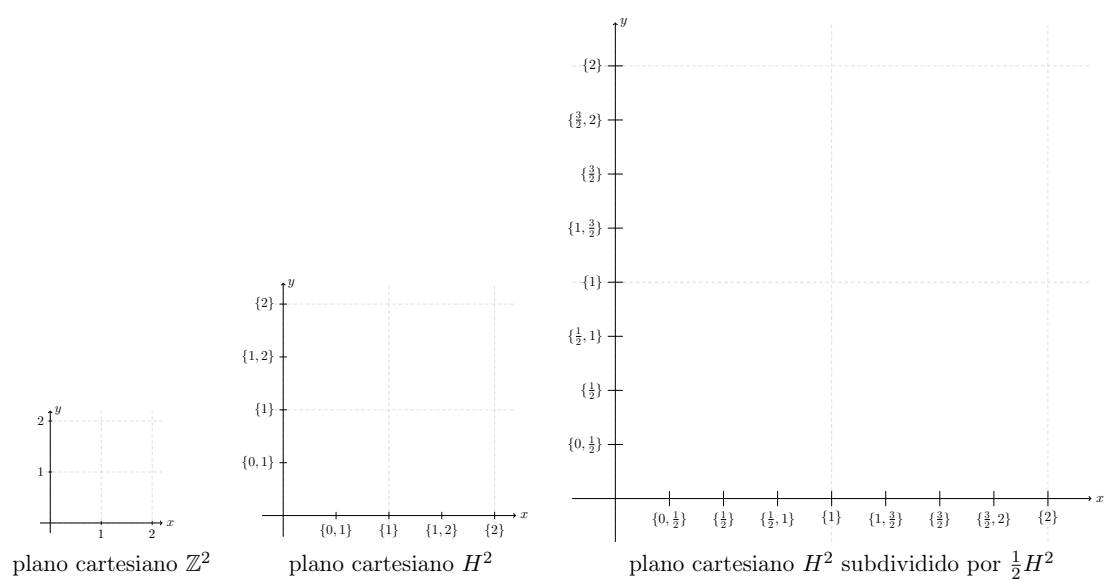

Figura 4.17: Exemplos de planos cartesiano dos conjuntos $\mathbb{Z}^{2}, H^{2}$ e $\frac{1}{2} H^{2}$. Note que, o domínio da função $F$ é uma grade retangular $X \subseteq \frac{1}{2} H^{2}$.

Para o espaço de valores (contradomínio) da imagem, usa-se subdivisões $Y=\frac{1}{2} H^{1}$, onde cada inteiro torna-se um único valor de $H_{1}^{0}$. Então, pode-se representar (interpolar) $f$ por meio de uma função valor-conjunto $F: X \rightsquigarrow Y$, definida por:

$$
\forall h \in X, F(h)= \begin{cases}f\left(m^{-1}(h)\right), & \text { se } f\left(m^{-1}(h)\right) \in \mathcal{D}, \\ \max \left\{F\left(h^{\prime}\right): h^{\prime} \in \operatorname{st}(\operatorname{cl}(h)) \cap \mathcal{D}\right\}, & \text { se } h \in \frac{1}{2} H_{1}^{2} \backslash \mathcal{D}, \\ \operatorname{span}\left\{F\left(h^{\prime}\right): h^{\prime} \in \operatorname{st}(h) \cap \mathcal{D}\right\}, & \text { se } h \in X \backslash \frac{1}{2} H_{1}^{2},\end{cases}
$$

onde o operador span devolve um par de elementos contendo o menor e o maior elemento do 
conjunto. Na Figura 4.18 é apresentado um exemplo de interpolação e no Algoritmo 4.4 é apresentada uma implementação proposta da Equação 4.9, onde o domínio $X$ é representado por inteiros $\{0,1, \ldots,|\mathcal{D}| \times 4-6\}$ e assim, por meio de aritmética modular são implementados os três casos da Equação 4.9. Note que os inteiros divisíveis por 2 são as 2 -faces, os divisíveis por 4 são as 2 -faces presentes em $\mathcal{D}$ e os ímpares são as 1 -faces e 0 -faces.

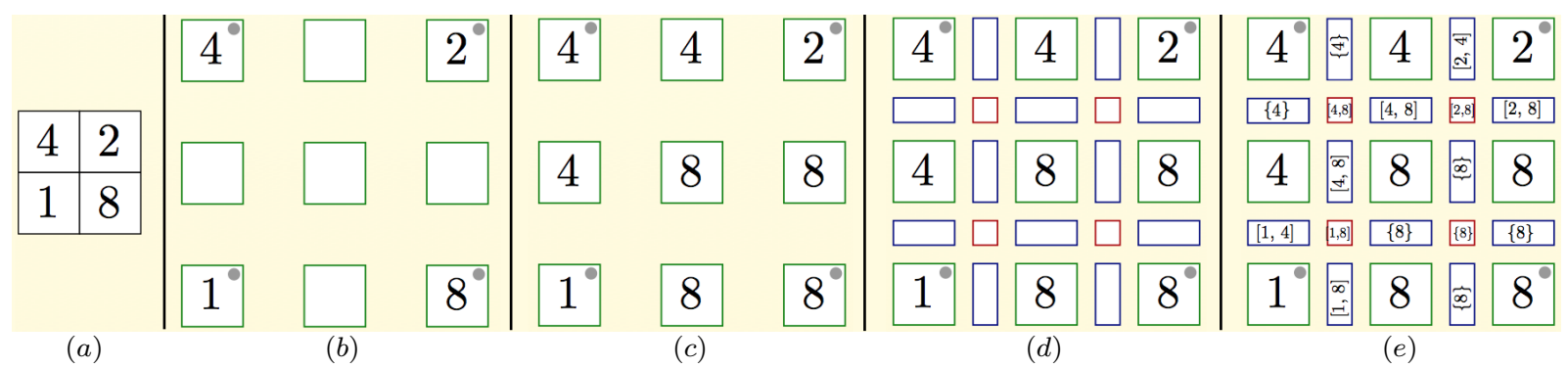

Figura 4.18: Figura adaptada de Géraud et al. (2013) contendo um exemplo de interpolação, onde (a) é a imagem de entrada; (b) é a primeira subdivisão do dominio e os elementos preenchidos é a aplicação de $f$ nos elementos de $\mathcal{D} ;(c)$ é a aplicação do operador max nos elementos que estão em $\frac{1}{2} H_{1}^{n} \backslash \mathcal{D} ;(d)$ é a segunda subdivisão de $\frac{1}{2} H^{2} ;(e)$ é a aplicação do operador span nos elementos de $X \backslash \frac{1}{2} H_{1}^{2}$.

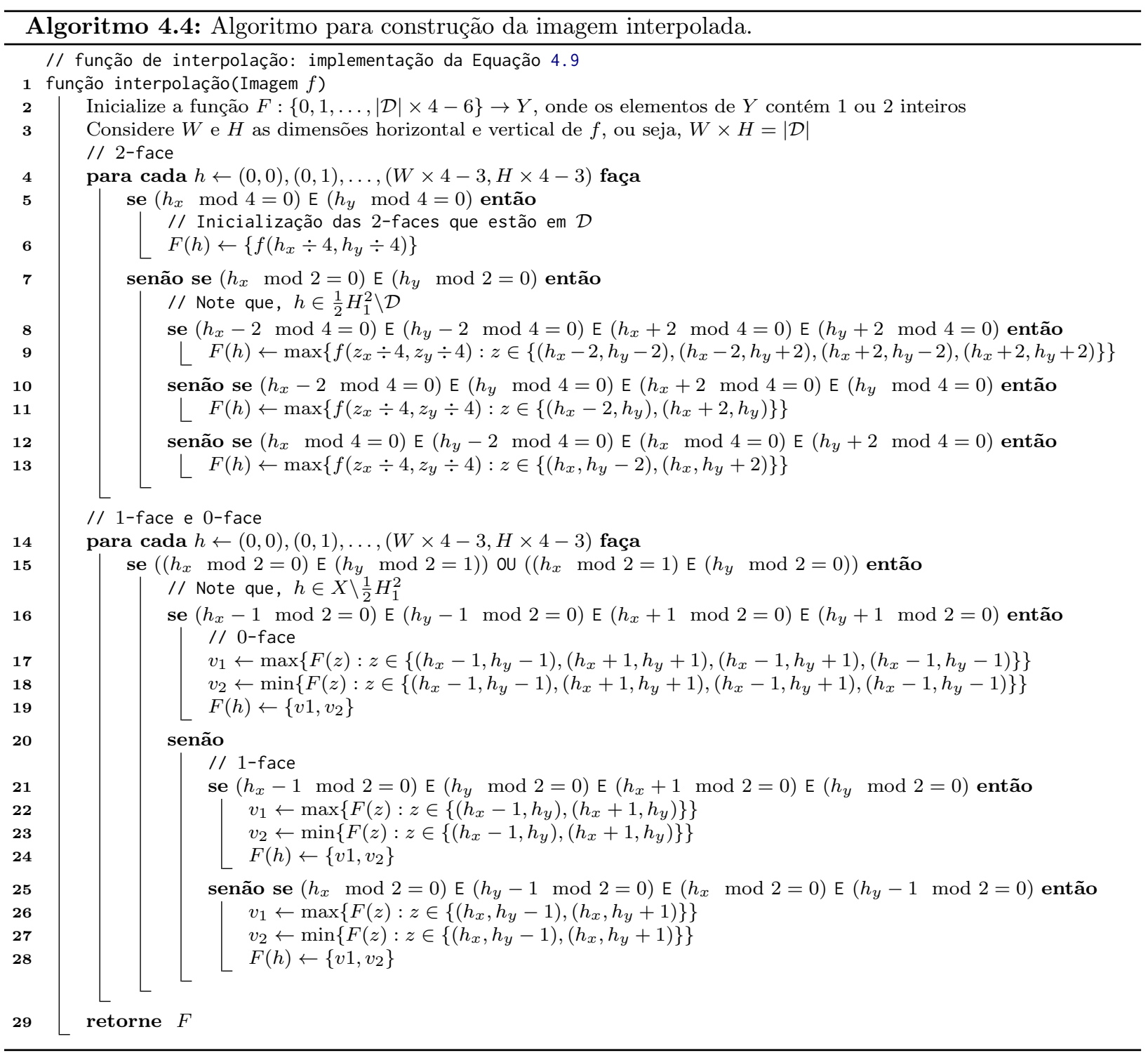


Com base na função $F$ pode-se definir noções de conjuntos de níveis saturados como feito por Najman e Géraud (2013). Aliás, Najman e Géraud (2013) mostra que $\mathcal{S} \mathcal{A} \mathcal{T}(f)=\mathcal{S} \mathcal{A} \mathcal{T}(F) \cap \mathbb{Z}^{2}$. Com base neste fato, Géraud et al. (2013) propôs um algoritmo, ver função ordenação-das-faces no Algoritmo 4.5, baseado em um processo de crescimento de regiões, para ordenar as faces de $F$ para em seguida construir a árvore de formas com algoritmo de Berger et al. (2007).

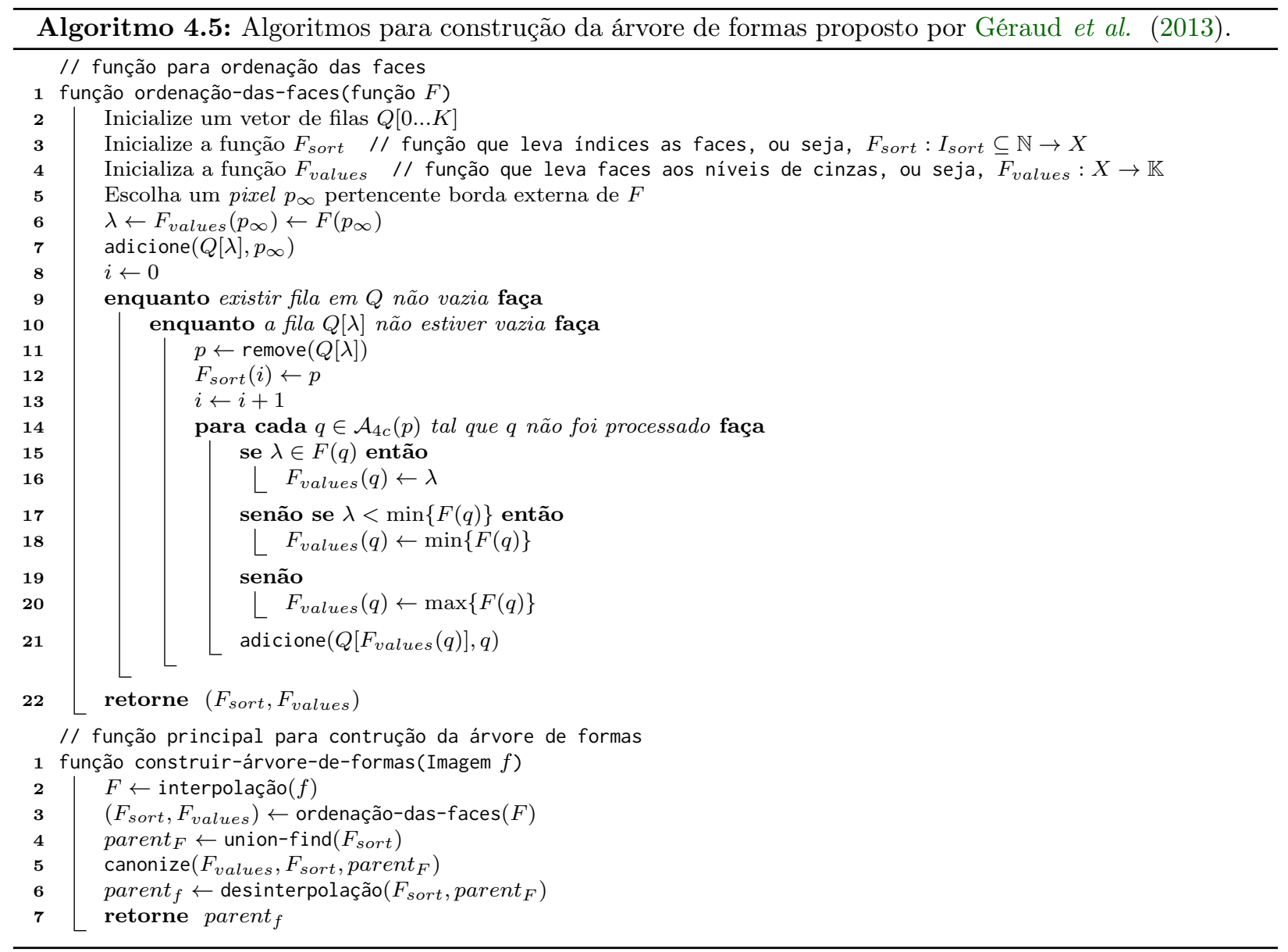

A função ordenação-das-faces utiliza um vetor de filas $Q$ para simular uma fila de prioridades. O algoritmo inicia a propagação em um pixel $p_{\infty}$ escolhido para representar a raiz da árvore. Geralmente este pixel pertence ao maior CC que intersecta a borda da imagem (ver sobre a escolha de $p_{\infty}$ em Monasse (2000)). No processo de crescimento de região, cada vez que uma face $p$ é visitada em uma prioridade (nível de cinza) $\lambda$, as faces $q$ da vizinhança de $p$ são inseridas na fila de prioridade $Q$ com uma prioridade determinada pela posição do seu valor em relação a $\lambda$. Se $\lambda \in F(q)$ então $q$ pertence a um conjunto de nível de valor $\lambda$ que também contém $p$ e assim $q$ é inserido em $Q$ com prioridade $\lambda$. Se $\forall k \in F(q), \lambda<k$ então o próximo nível (prioridade) a ser processado após $\lambda$ é $m=\min \{\lambda: \lambda \in F(q)\}$ e assim $q$ é inserido na fila com prioridade $m$. Analogamente, se $\forall k \in F(q), \lambda>k$ então o próximo nível a ser processado após $\lambda$ é $m=\max \{\lambda: \lambda \in F(q)\}$ e assim $q$ é inserido na fila com prioridade $m$. Esta função ordenação-das-faces desempenha dois papeis, a ordenação $F_{\text {sort }}$ das faces e a criação de uma imagem da interpolação $F_{\text {values }}$ contendo um único valor por pixel. Mais detalhes sobre este algoritmo podem ser encontrados em Géraud et al. (2013) e Crozet e Géraud (2014). Então, para construir a árvore de formas, basta considerar a função construir-árvore-de-formas do Algoritmo 4.5, onde (1) é interpolada e ordenada a imagem de entrada. Depois, (2) é construída a árvore por union-find e finalmente são removidos os pixels 
desnecessários da árvore de formas. Note que as funções interpolação e ordenação-das-faces têm a mesma complexidade computacional, isto é, $\mathcal{O}(16 \mathcal{D})$. Na Figura 4.19 é apresentado um exemplo de uma computação (entrada e saída) do Algoritmo 4.5.

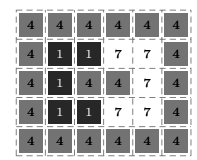

imagem $f$
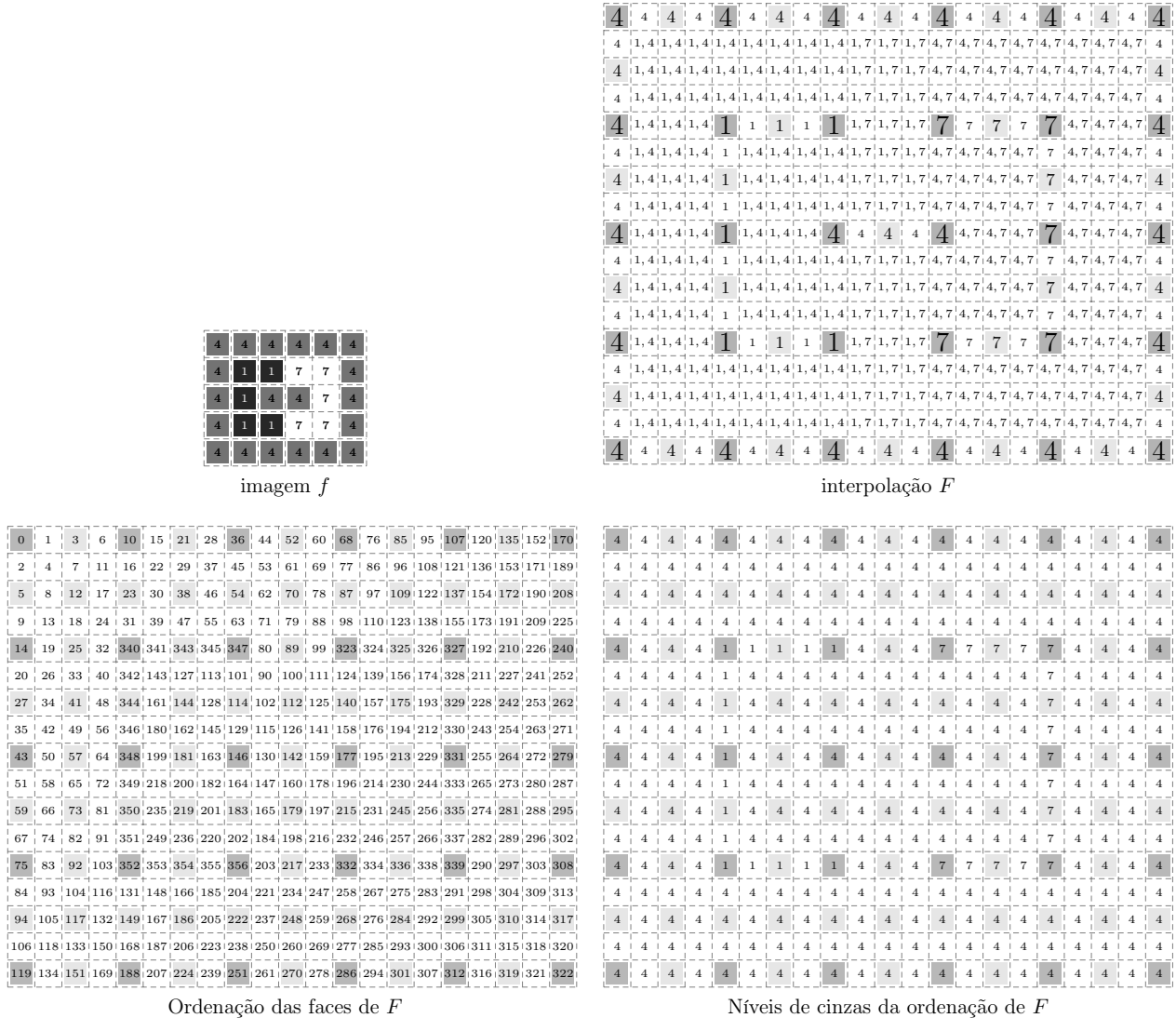

Ordenação das faces de $F$
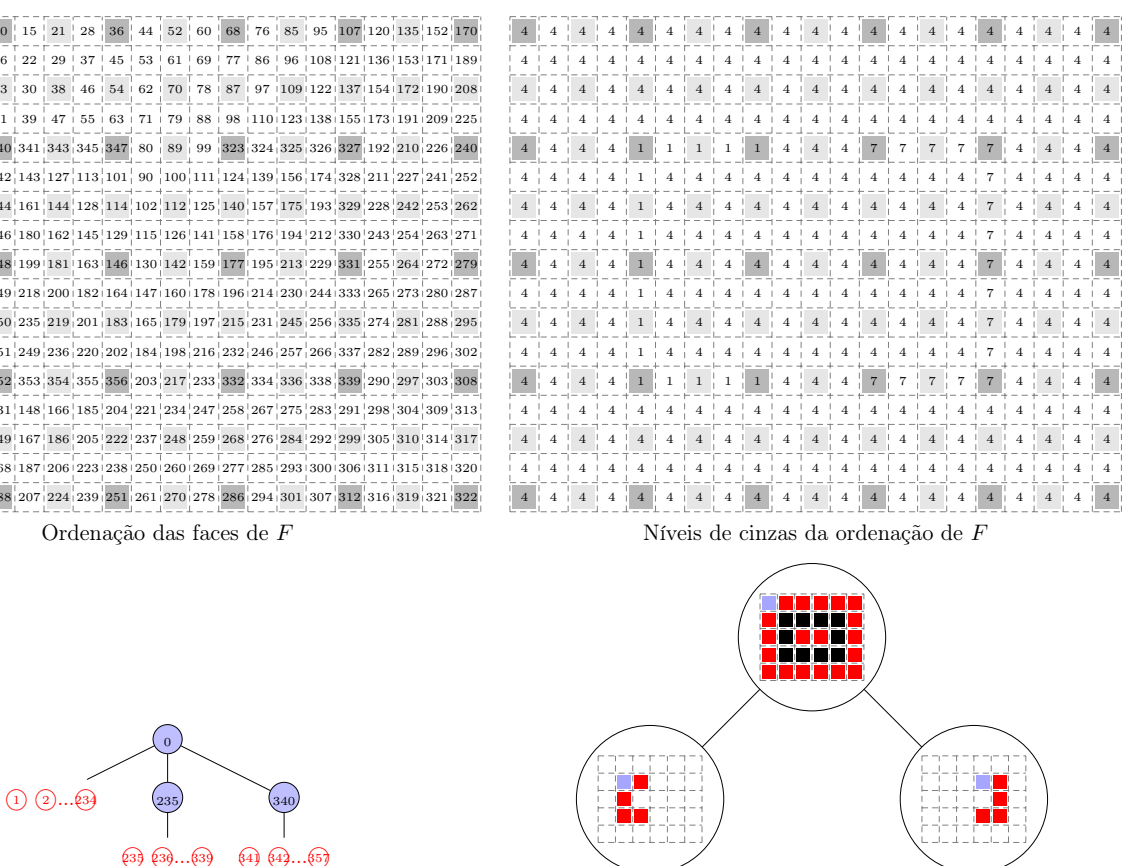

Relação de parentesco da função parent canonizada

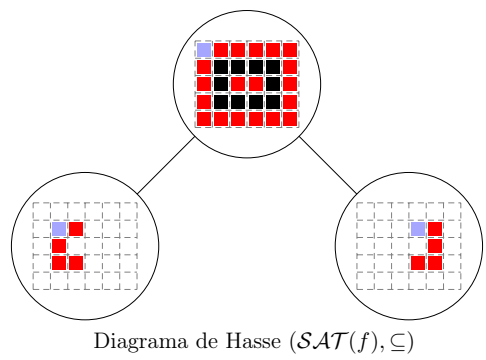

Figura 4.19: Exemplo de construção da árvore de formas.

\subsubsection{Reconstrução de árvores podadas}

Como apresentado na Equação 4.4, uma imagem pode ser reconstruída a partir dos seus conjuntos de níveis. Agora, pretende-se reconstruir uma imagem a partir de sua árvore. Isto leva à definir funções level $_{\mathcal{L}}: \mathcal{L}(f) \rightarrow \mathbb{K}$, level $\mathfrak{\mathcal { U }}: \mathcal{U}(f) \rightarrow \mathbb{K}$ e level $_{\mathcal{S} \mathcal{A} \mathcal{T}}: \mathcal{S} \mathcal{A} \mathcal{T}(f) \rightarrow \mathbb{K}$ derivadas da Equação 4.4 que mapeiam vértices em níveis de cinzas, como a seguir:

$$
\begin{aligned}
& \text { level }_{\mathcal{L}}(C)=\min \left\{\lambda-1: C \in \mathcal{C C}\left(\mathcal{X}_{\downarrow}^{\lambda}(f)\right), \lambda \in \mathbb{K}\right\} \\
& \text { level }_{\mathcal{U}}(C)=\max \left\{\lambda: C \in \mathcal{C C}\left(\mathcal{X}_{\lambda}^{\uparrow}(f)\right), \lambda \in \mathbb{K}\right\} \text { e } \\
& \text { level }_{\mathcal{S} \mathcal{A} \mathcal{T}}(C)=f(y) \text { tal que } y \in \arg \max \left\{\left|\mathcal{S C}\left(\mathcal{T}_{f}, x\right)\right|: x \in C\right\}
\end{aligned}
$$

Obviamente, a função level para as versões estendidas da min-tree e max-tree é simplesmente

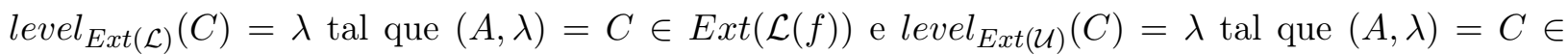


$\operatorname{Ext}(\mathcal{U}(f))$, respectivamente. Por simplicidade de notação, a partir de agora adota-se a nomenclatura level para todas essas funções. Também, é denotado por $\mathcal{T}_{f}$ uma árvore construída a partir de uma imagem $f \in \mathcal{F}(\mathcal{D})$. Dessa forma, partindo da árvore $\mathcal{T}_{f}$ pode-se reconstruir a imagem $f$ por:

$$
\forall p \in \mathcal{D}, f(p)=\operatorname{level}\left(\mathcal{S C}\left(\mathcal{T}_{f}, p\right)\right)
$$

Neste caso, esta operação é escrita como: $f=\operatorname{Rec}\left(\mathcal{T}_{f}\right)$. Em particular, se $f \in \mathcal{F}(\mathcal{D})$ é uma imagem obtida por $\operatorname{Rec}((\mathcal{L}(f), \subseteq))$ (respectivamente, $\operatorname{Rec}((\mathcal{U}(f), \subseteq))$ e $\operatorname{Rec}((\mathcal{S} \mathcal{A} \mathcal{T}(f), \subseteq)))$, então chama-se esta operação por reconstrução inferior (respectivamente, superior e por formas).

Portanto, seguindo estas definições, se $\mathcal{T}_{f}$ é a árvore de uma imagem $f \in \mathcal{F}(\mathcal{D})$, então dizemos que $\mathcal{T}_{g}$ é a versão podada da árvore $\mathcal{T}_{f}$ se, e somente se, $\mathcal{T}_{g}=\mathcal{P}$ oda $\left(\mathcal{T}_{f}\right)$ e $g=\operatorname{Rec}\left(\mathcal{T}_{g}\right)$. Assim, $g$ é a imagem de referência para aplicação da função level para os vértices de $\mathcal{T}_{g}$. Na Figura 4.20 são apresentados exemplos das operações de podas e reconstruções para uma dada imagem.

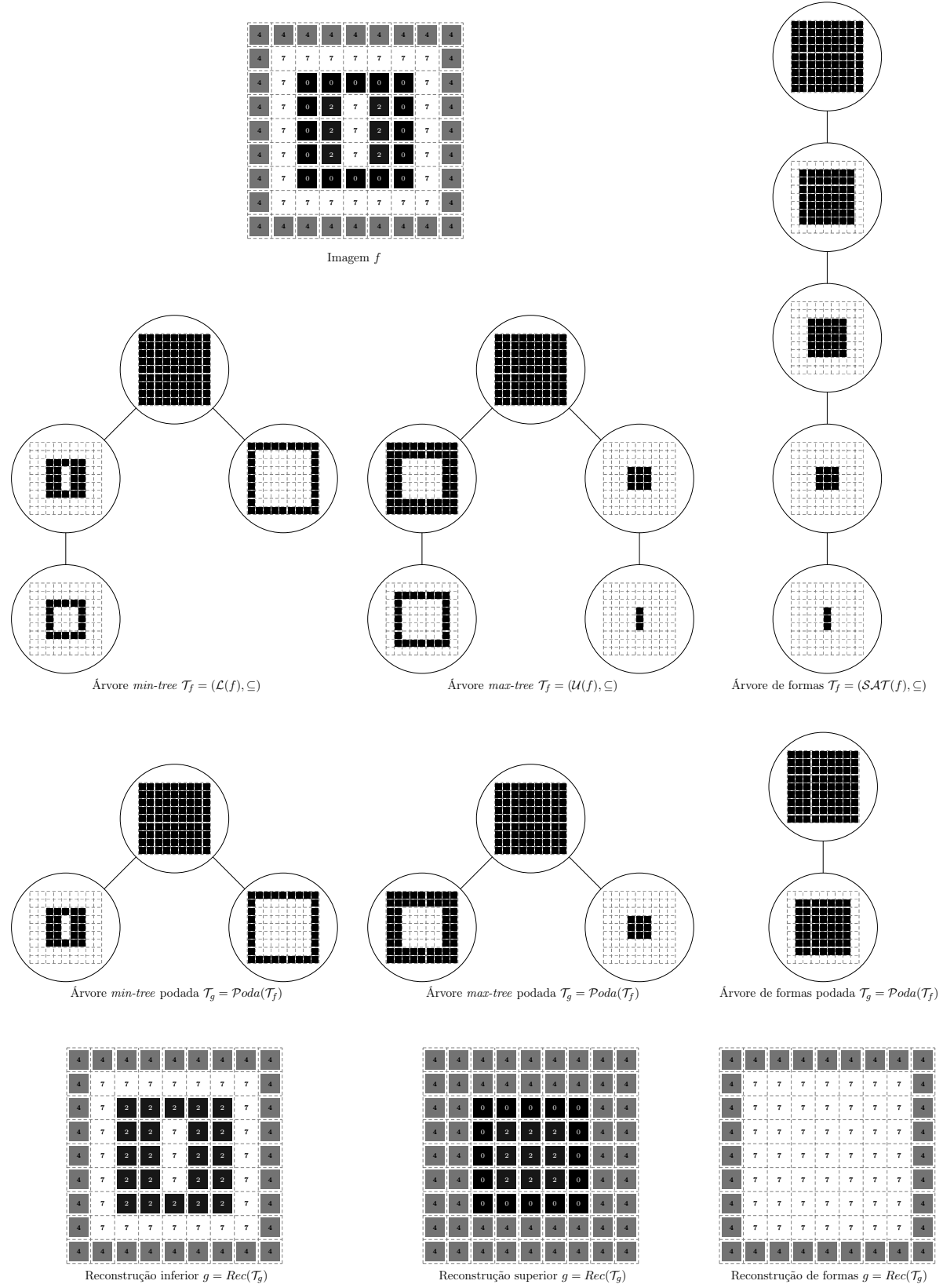

Figura 4.20: Exemplos das operações de podas e reconstruções. 
50 REPRESENTAÇÕES DE IMAGENS POR MEIO DE ÁRVORES BASEADAS EM CONJUNTOS DE NÍVEIS 


\section{Parte II}

\section{Últimos levelings: conceitos, propriedades e algoritmos}

\section{Resumo}

Esta parte da tese é dedicada à apresentação da classe de operadores residuais denominada últimos levelings, a qual consiste de poderosos operadores residuais estendidos, definidos a partir de espaços de escalas baseados em levelings. Um operador desta classe analisa a evolução dos valores residuais presentes em dois operadores consecutivos de um espaço de escala e os maiores valores residuais são considerados. Os valores residuais revelam uma importante informação sobre o contraste de uma imagem. Além dos valores residuais, outras informações associadas com eles podem ser obtidas no momento da extração residual, tais como propriedades das regiões que produziram os valores residuais. Alguns exemplos de operadores desta classe conhecidos na literatura incluem a última abertura (resp. fechamento) por atributo (Retornaz e Marcotegui, 2007) e o último filtro por grão (Alves e Hashimoto, 2014). Eles têm sido usados com sucesso em uma etapa de pré-processamento em diversas aplicações, como por exemplo, a localização de texto em imagens de cenas (Alves e Hashimoto, 2010; Retornaz e Marcotegui, 2007), a segmentação de fachadas de edifícios (Hernandez e Marcotegui, 2011), a restauração de imagens de documentos históricos (Meyer, 2010), entre outras.

Ainda, nesta parte da tese são apresentadas as principais contribuições originais desta pesquisa, as quais incluem: $(i)$ uma representação compacta e eficiente de um espaço de escala baseado em levelings por meio de uma árvore (Capítulo 5); (ii) definições, conceitos, algoritmos, propriedades e relações de operadores conhecidos na literatura com os últimos levelings (Capítulo 6); (iii) estratégias para construções de operadores últimos levelings (Capitulo 7). 


\title{
Representações de espaço de escala baseado em levelings através de hierarquias dos conjuntos de níveis
}

\begin{abstract}
Resumo do capítulo
Em muitas aplicações no campo de análise e processamento de imagens, os objetos de interesse precisam ser detectados, mensurados, segmentados ou reconhecidos em uma imagem, em geral, não pertencem a uma escala fixa, mas sim a muitas escalas. Nesta situação, diversas representações de espaço de escala têm sido desenvolvidas nas últimas décadas, como por exemplo, o espaço de escala por meio encadeamento de levelings mostrado na Seção 3.6. Neste sentido, é apresentada nesta tese um espaço de escala baseado em levelings por meio de uma hierarquia dos conjuntos de niveis, mais especificamente, por meio das árvores de componentes e das árvores de formas. Primeiramente, é demostrado que reconstruções de árvores podadas são levelings, depois são associadas reconstruções de árvores podadas com duas categorias de operadores: os baseados em imagens marcadoras e os baseados em atributos crescentes. Por fim, é mostrado que uma árvore representa um espaço de escala que pode ser construído por meio de podas sucessivas na árvore.
\end{abstract}

Vale salientar que neste capítulo são apresentadas as primeiras contribuições originais desta tese (Alves et al., 2015), onde são mostrados que: (i) reconstruções superiores, inferiores e por formas são operadores levelings; (ii) filtros conexos baseados em imagens marcadoras podem ser obtidos por podas em árvores; (iii) Por fim, é mostrado que um espaço de escala (extensivo, anti-extensivo ou auto-dual) baseado em levelings pode ser representado por uma árvore (de componentes ou de formas) e construído por meio de podas sucessivas baseadas em imagens marcadoras ou atributos.

\subsection{Relações entre reconstruções de árvores podadas e operadores levelings}

No Capítulo 3 foram apresentados conceitos sobre operadores levelings e no Capítulo 4 foram apresentados conceitos sobre representação de imagens por meio de árvores de componentes e de formas. Além disso, foram estabelecidas as operações de podas e reconstruções de árvores podadas. Nesta seção, são apresentadas relações entre reconstruções de árvores podadas (ou seja, reconstruções inferiores, superiores e por formas) com os operadores levelings. Primeiramente, são mostradas 
as relações entre árvores de componentes e depois árvores de formas, com os operadores levelings.

\section{Relações entre reconstruções superiores (respectivamente, inferiores) e operadores le- velings}

Seja $\mathcal{T}_{f}$ uma árvore max-tree (respectivamente, min-tree) construída a partir de uma imagem $f \in \mathcal{F}(\mathcal{D})$ e considere $\mathcal{T}_{g}$ a árvore obtida por uma operação de poda em $\mathcal{T}_{f}$, ou seja, $\mathcal{T}_{g}=\mathcal{P}$ oda $\left(\mathcal{T}_{f}\right)$. Logo, por meio das definições de poda e menores componentes (ver Definições 4.10 e 4.14) podese concluir que $\forall p \in \mathcal{D}, \mathcal{S C}\left(\mathcal{T}_{f}, p\right) \subseteq \mathcal{S C}\left(\mathcal{T}_{g}, p\right)$ e pela definição de reconstrução de árvore podada (ver Seção 4.2.3) temos ainda que $\operatorname{level}\left(\mathcal{S C}\left(\mathcal{T}_{f}, p\right)\right) \geq \operatorname{level}\left(\mathcal{S C}\left(\mathcal{T}_{g}, p\right)\right.$ ) (respectivamente, level $\left.\left(\mathcal{S C}\left(\mathcal{T}_{f}, p\right)\right) \leq \operatorname{level}\left(\mathcal{S C}\left(\mathcal{T}_{g}, p\right)\right)\right)$, graças à ordem bem definida dos conjuntos de níveis. Dessa forma, temos que $\operatorname{Rec}\left(\mathcal{T}_{f}\right) \geq \operatorname{Rec}\left(\mathcal{T}_{g}\right)$ (respectivamente, $\operatorname{Rec}\left(\mathcal{T}_{f}\right) \leq \operatorname{Rec}\left(\mathcal{T}_{g}\right)$ ) o que mostra que reconstruções superiores (respectivamente, inferiores) são anti-extensivas (respectivamente, extensivas). Estas colocações dão origem à Proposição 5.1.

Proposição 5.1. Sejam $\mathcal{T}=(\mathcal{U}(f), \subseteq)$ a árvore max-tree (respectivamente, min-tree $\mathcal{T}=(\mathcal{L}(f), \subseteq$ )) de uma imagem $f \in \mathcal{F}(\mathcal{D})$ e $(p, q) \in \mathcal{A}$. Então, $f(p)>f(q)$ (respectivamente, $f(p)<f(q)$ ) se, e somente se, $\mathcal{S C}(\mathcal{T}, p) \subset \mathcal{S C}(\mathcal{T}, q)$.

\section{Prova:}

$\Longrightarrow$ Como $f(p)>f(q)$, então por definição de conjunto de níveis, tem-se: $\mathcal{X}_{f(p)}^{\uparrow}(f)=\{x \in \mathcal{D}:$ $f(x) \geq f(p)>f(q)\} \subset \mathcal{X}_{f(q)}^{\uparrow}(f)=\{x \in \mathcal{D}: f(x) \geq f(q)\} . \operatorname{Logo}, p \in \mathcal{S C}(\mathcal{T}, p) \subseteq \mathcal{X}_{f(p)}^{\uparrow}(f) \subset$ $\mathcal{X}_{f(q)}^{\uparrow}(f)$. Também temos que, $q \in \mathcal{S C}(\mathcal{T}, q) \subseteq \mathcal{X}_{f(q)}^{\uparrow}(f)$. Dessa forma, temos: $(i) \mathcal{S C}(\mathcal{T}, p)$ é um CC de $\mathcal{X}_{f(p)}^{\uparrow}(f)$ contendo $p ;(i i) \mathcal{S C}(\mathcal{T}, q)$ é um CC de $\mathcal{X}_{f(q)}^{\uparrow}(f)$ contendo $q ;(i i i)(p, q)$ são adjacentes, ou seja, $(p, q) \in \mathcal{A}$; e $(i v) f(p)>f(q)$. Então, $p \in \mathcal{S C}(\mathcal{T}, q)$ e consequentemente $\mathcal{S C}(\mathcal{T}, p) \subset \mathcal{S C}(\mathcal{T}, q)$, pois existem caminhos em $\mathcal{S C}(\mathcal{T}, q)$ para todos os pixels de $\mathcal{S C}(\mathcal{T}, p)$ passando por $p$.

$\Longleftarrow$ Por definição de conjuntos de níveis, temos que $\mathcal{S C}(\mathcal{T}, p) \in \mathcal{C C}\left(\mathcal{X}_{f(p)}^{\uparrow}(f)\right) \subseteq \mathcal{U}(f)$ e $\mathcal{S C}(\mathcal{T}, q) \in$ $\mathcal{C C}\left(\mathcal{X}_{f(q)}^{\uparrow}(f)\right) \subseteq \mathcal{U}(f)$ pois $\mathcal{X}_{f(p)}^{\uparrow}(f)$ e $\mathcal{X}_{f(q)}^{\uparrow}(f)$ são os menores conjuntos de níveis contendo os pixels $p$ e $q$, respectivamente. Assim, $\operatorname{como} \mathcal{S C}(\mathcal{T}, p) \subset \mathcal{S C}(\mathcal{T}, q)$ segue que $\mathcal{X}_{f(p)}^{\uparrow}(f) \subset \mathcal{X}_{f(q)}^{\uparrow}(f)$, pois os conjuntos de níveis são aninhados pela relação de inclusão (ver Equação 4.3). Logo, por definição dos conjuntos de níveis, temos que $\mathcal{X}_{f(p)}^{\uparrow}(f)=\{x \in \mathcal{D}: f(x) \geq f(p)>f(q)\} \subset$ $\mathcal{X}_{f(q)}^{\uparrow}(f)=\{x \in \mathcal{D}: f(x) \geq f(q)\}$. Portanto, $f(p)>f(q)$.

A prova para os conjuntos de níveis inferiores segue de forma similar.

Estes fatos mostram que, se $\mathcal{T}_{g}$ é obtida por uma operação de poda de uma árvore max-tree (respectivamente, min-tree) $\mathcal{T}_{f}$, então a imagem $g=\operatorname{Rec}\left(\mathcal{T}_{g}\right)$ é um leveling da imagem $f=\operatorname{Rec}\left(\mathcal{T}_{f}\right)$, pois para todo $(p, q) \in \mathcal{A}$, a seguinte condição é sempre verdadeira: $g(p)>g(q) \Rightarrow f(p) \geq g(p)>$ $g(q)=f(q)$ (respectivamente, $g(p)>g(q) \Rightarrow f(p)=g(p)>g(q) \geq f(q)$ ). Isso, pode ser constatado pelos casos mostrados na Figura 5.1 ou ainda pela prova mostrada na Proposição 5.2.

Proposição 5.2. Reconstruções superiores (respectivamente, inferiores) são levelings.

Prova: Sejam $f \in \mathcal{F}(\mathcal{D})$ uma imagem e $\mathcal{T}_{f}$ a árvore max-tree de $f$. Então, $g \in \mathcal{F}(\mathcal{D})$ é uma reconstrução superior de $f$ se, e somente se, $g=\operatorname{Rec}\left(\mathcal{T}_{g}\right)$ tal que $\mathcal{T}_{g}=\operatorname{Poda}\left(\mathcal{T}_{f}\right)$. Para mostrar que $g$ é um leveling de $f$, basta verificar se para todo $(p, q) \in \mathcal{A}$, a definição de levelings é verdadeira. 
Então, considere dois casos, quando $f(p)=f(q)$ e $f(p) \neq f(q)$. No primeiro caso, $g$ satisfaz a definição de levelings por vacuidade, pois $(p, q)$ pertence a uma mesma zona plana de $f$ e consequentemente no mesmo vértice em $\mathcal{T}_{f}$ e portanto $g(p)=g(q)$. No segundo caso, temos que $\mathcal{S C}\left(\mathcal{T}_{f}, p\right)$ e $\mathcal{S C}\left(\mathcal{T}_{f}, q\right)$ são comparáveis, pois $(p, q) \in \mathcal{A}, \mathcal{S C}\left(\mathcal{T}_{f}, p\right) \cap \mathcal{S C}\left(\mathcal{T}_{f}, q\right) \neq \emptyset$ e graça ao Corolário 4.13, dois vértices são disjuntos ou comparáveis. Logo, a operação de poda que gera $\mathcal{T}_{g}$ a partir de $\mathcal{T}_{f}$ pode: (1) ou um dos dois vértices é podado (como ilustrado na Figura 5.1 - casos $1 a$ e 1b); (2) ou ambos $\mathcal{S C}\left(\mathcal{T}_{f}, p\right)$ e $\mathcal{S C}\left(\mathcal{T}_{f}, q\right)$ são podados ou preservados (como ilustrado na Figura 5.1 - casos $2 a$ e $2 b$ ). Analisando este casos, temos:

1. Se podar um dos dois vértices, então sem perda de generalidade suponha que $\mathcal{S C}\left(\mathcal{T}_{f}, p\right)$ é podado e $\mathcal{S C}\left(\mathcal{T}_{f}, q\right)$ é preservado. Assim, $\mathcal{S C}\left(\mathcal{T}_{f}, p\right) \subset \mathcal{S C}\left(\mathcal{T}_{g}, p\right)$ e $\mathcal{S C}\left(\mathcal{T}_{g}, q\right)=\mathcal{S C}\left(\mathcal{T}_{f}, q\right)$. Logo, ou $\mathcal{S C}\left(\mathcal{T}_{f}, p\right) \subset \mathcal{S C}\left(\mathcal{T}_{g}, p\right) \subset \mathcal{S C}\left(\mathcal{T}_{g}, q\right)=\mathcal{S C}\left(\mathcal{T}_{f}, q\right)$ (ver Fig. 5.1 - caso $\left.1 a\right)$, ou $\mathcal{S C}\left(\mathcal{T}_{f}, p\right) \subset$ $\mathcal{S C}\left(\mathcal{T}_{g}, p\right)=\mathcal{S C}\left(\mathcal{T}_{g}, q\right)=\mathcal{S C}\left(\mathcal{T}_{f}, q\right)$ (ver Fig. 5.1 - caso 1b). Portanto, pela Proposição 5.1, segue que: ou $f(p) \geq g(p)>g(q)=f(q)$, ou $f(p)=g(p)=g(q)=f(q)$ o que satisfaz a Definição 3.3 de levelings.

2. Se ambos $\mathcal{S C}\left(\mathcal{T}_{f}, p\right)$ e $\mathcal{S C}\left(\mathcal{T}_{f}, q\right)$ são preservados ou podados. Então, ou $\mathcal{S C}\left(\mathcal{T}_{f}, p\right)=\mathcal{S C}\left(\mathcal{T}_{g}, p\right)$ e $\mathcal{S C}\left(\mathcal{T}_{f}, q\right)=\mathcal{S C}\left(\mathcal{T}_{g}, q\right)$ e por consequência segue que: ou $f(p)=g(p)>g(q)=f(q)$ (ver Fig. 5.1 - caso $2 a)$, ou $\mathcal{S C}\left(\mathcal{T}_{g}, p\right)=\mathcal{S C}\left(\mathcal{T}_{g}, q\right)$ e consequentemente $g(p)=g(q)$ (ver Fig. 5.1 casos $2 a$ e $2 b)$ o que satisfaz a Definição 3.3 de levelings.

Portanto, reconstruções superiores são levelings. A prova para reconstruções inferiores segue de forma análoga.
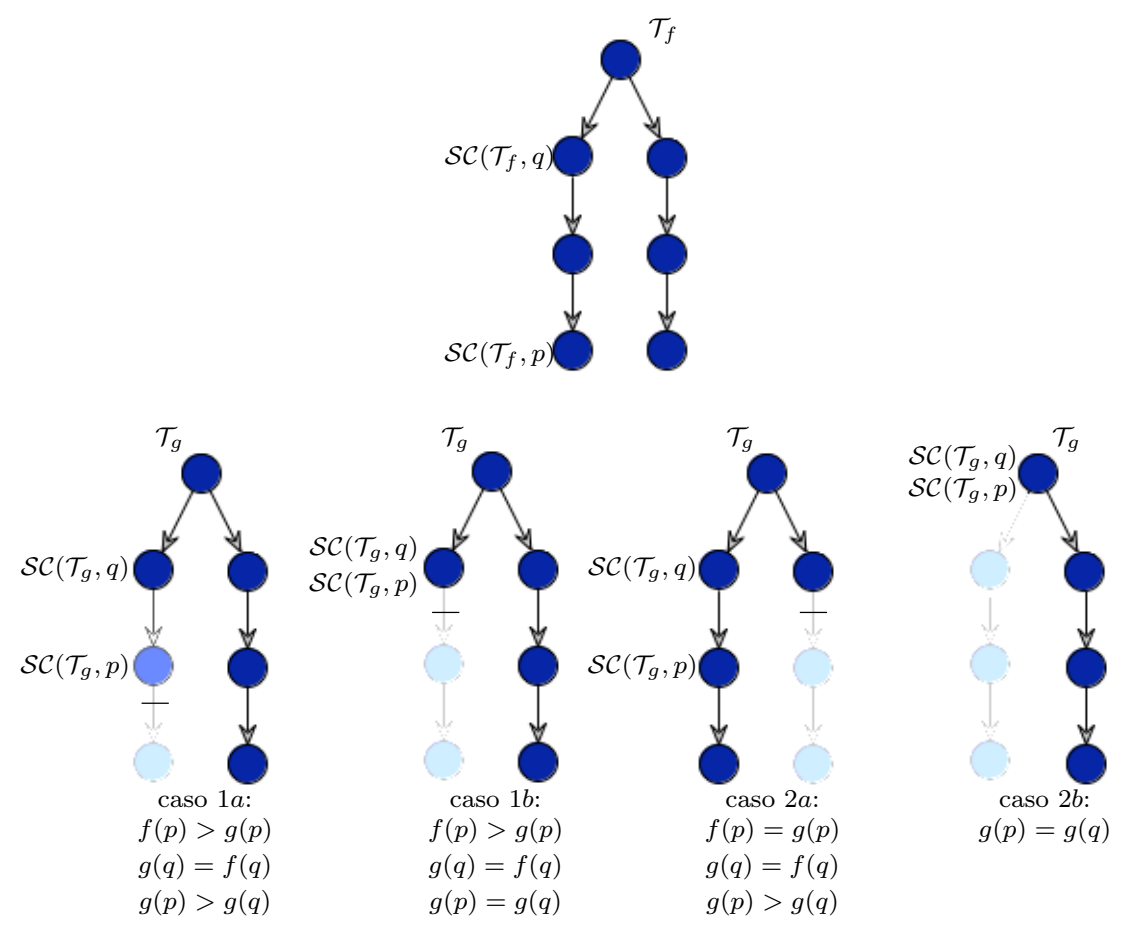

Figura 5.1: Ilustrações das configurações de podas em uma árvore max-tree.

De fato, este resultado não é novo e já foi mencionado por muito autores, como por exemplo: Salembier e Wilkinson (2009), Garrido (2002) e Xu (2013). No entanto, temos um novo resultado quando consideramos a versão estendida da max-tree (respectivamente, min-tree). Assim, como 
é garantido pelo Teorema 5.3, reconstruções superiores (respectivamente, inferiores) por meio da versão estendida da max-tree (respectivamente, min-tree) são equivalentes aos operadores levelings anti-extensivos (respectivamente, extensivos).

Teorema 5.3. Reconstruções superiores (respectivamente, inferiores) e levelings anti-extensivos (respectivamente, extensivos) são equivalentes.

Prova: Sejam $f \in \mathcal{F}(\mathcal{D})$ uma imagem e $\mathcal{T}_{f}=(\operatorname{Ext}(\mathcal{U}(f))$, $)$ a versão estendida da max-tree da imagem $f$. Assim, temos que: $g \in \mathcal{F}(\mathcal{D})$ é uma reconstrução superior de $f$

$$
\begin{aligned}
& \Longleftrightarrow g=\operatorname{Rec}\left(\mathcal{T}_{g}\right) \text { tal que } \mathcal{T}_{g}=\operatorname{Poda}\left(\mathcal{T}_{f}\right) \\
& \Longleftrightarrow g \leq f \mathrm{e} \forall(p, q) \in \mathcal{A} \text {, } \\
& \left\{\begin{array}{l}
\text { ou } \mathcal{S C}\left(\mathcal{T}_{f}, p\right) \sqsubseteq \mathcal{S C}\left(\mathcal{T}_{f}, q\right) \sqsubseteq \mathcal{S C}\left(\mathcal{T}_{g}, p\right)=\mathcal{S C}\left(\mathcal{T}_{g}, q\right) \\
\text { ou } \mathcal{S C}\left(\mathcal{T}_{f}, p\right) \sqsubseteq \mathcal{S C}\left(\mathcal{T}_{g}, p\right) \sqsubseteq \mathcal{S C}\left(\mathcal{T}_{f}, q\right)=\mathcal{S C}\left(\mathcal{T}_{g}, q\right)
\end{array}\right. \\
& \Longleftrightarrow g \leq f \text { e } \forall(p, q) \in \mathcal{A}, \mathcal{S C}\left(\mathcal{T}_{g}, p\right) \sqsubset \mathcal{S C}\left(\mathcal{T}_{g}, q\right) \\
& \Rightarrow\left\{\begin{aligned}
\mathcal{S C}\left(\mathcal{T}_{f}, p\right) & \sqsubseteq \mathcal{S C}\left(\mathcal{T}_{g}, p\right) \\
\mathrm{e} & \\
\mathcal{S C}\left(\mathcal{T}_{g}, q\right) & =\mathcal{S C}\left(\mathcal{T}_{f}, q\right)
\end{aligned}\right. \\
& \Longleftrightarrow \quad g \leq f \text { e } \forall(p, q) \in \mathcal{A}, \text { level }\left(\mathcal{S C}\left(\mathcal{T}_{g}, p\right)\right)>\operatorname{level}\left(\mathcal{S C}\left(\mathcal{T}_{g}, q\right)\right) \\
& \Rightarrow\left\{\begin{aligned}
\text { level }\left(\mathcal{S C}\left(\mathcal{T}_{f}, p\right)\right) & \geq \operatorname{level}\left(\mathcal{S C}\left(\mathcal{T}_{g}, p\right)\right) \\
& \mathrm{e} \\
\operatorname{level}\left(\mathcal{S C}\left(\mathcal{T}_{g}, q\right)\right. & =\operatorname{level}\left(\mathcal{S C}\left(\mathcal{T}_{f}, q\right)\right)
\end{aligned}\right. \\
& \Longleftrightarrow g \leq f \text { e } \forall(p, q) \in \mathcal{A}, g(p)>g(q) \Rightarrow f(p) \geq g(p) \text { e } g(q)=f(q) \\
& \Longleftrightarrow \quad g \text { é um leveling anti-extensivo de } f \text {. }
\end{aligned}
$$

A prova para a equivalência de levelings extensivos e reconstruções inferiores segue de maneira similar.

\section{Relações entre reconstruções de formas e operadores levelings}

Para estabelecer relações entre reconstruções por formas e operadores levelings é necessário conhecer as relações entre os pixels adjacentes e os vértices da árvore de formas. Neste sentido, as Proposições 5.4, 5.5, 5.6 e 5.8 ajudam a entender como os pixels adjacentes estão relacionados na árvore. Assim, a Proposição 5.4 é um corolário do Teorema 2.16 apresentado por Caselles e Monasse (2010), a Proposição 5.5 é uma consequência direta da Proposição 5.1 e a Proposição 5.8 é uma consequência direta da Proposição 5.6.

Proposição 5.4. Seja $\mathcal{T}_{f}$ a árvore de formas construída a partir de uma imagem $f \in \mathcal{F}(\mathcal{D})$. $S e(p, q) \in \mathcal{A}$ tal que $f(p) \neq f(q)$ então duas formas $\mathcal{S C}\left(\mathcal{T}_{f}, p\right)$ e $\mathcal{S C}\left(\mathcal{T}_{f}, q\right)$ são comparáveis ou disjuntas.

Prova: Por definição, level $\left(\mathcal{S C}\left(\mathcal{T}_{f}, p\right)\right)=f(p)$ e level $\left(\mathcal{S C}\left(\mathcal{T}_{f}, q\right)\right)=f(q)$. Portanto, como $f(p) \neq$ $f(q)$ então segue que $\mathcal{S C}\left(\mathcal{T}_{f}, p\right) \neq \mathcal{S C}\left(\mathcal{T}_{f}, q\right)$. Assim, o Teorema 2.16 de Caselles e Monasse (2010) garantem que duas formas em $\mathcal{T}_{f}$ são comparáveis ou disjuntas.

Proposição 5.5. Seja $\mathcal{T}_{f}$ a árvore de formas construída a partir de uma imagem $f \in \mathcal{F}(\mathcal{D})$. Se $(p, q) \in \mathcal{A}$ tal que $\mathcal{S C}\left(\mathcal{T}_{f}, p\right) \subset \mathcal{S C}\left(\mathcal{T}_{f}, q\right)$ e ambos $\mathcal{S C}\left(\mathcal{T}_{f}, p\right)$ e $\mathcal{S C}\left(\mathcal{T}_{f}, q\right)$ pertencem a $\mathcal{S} \mathcal{A} \mathcal{T}_{\mathcal{U}}(f)$ (respectivamente, $\mathcal{S} \mathcal{A}_{\mathcal{L}}(f)$ ), então $f(p)>f(q)$ (respectivamente, $f(p)<f(q)$ ).

Prova: Por definição do conjunto $\mathcal{S} \mathcal{A} \mathcal{T}_{\mathcal{U}}(f)$, segue que existem conjuntos $P$ e $Q$ pertencentes a $\mathcal{U}(f)$ tal que $\mathcal{S C}\left(\mathcal{T}_{f}, p\right)=\operatorname{sat}(P)$ e $\mathcal{S C}\left(\mathcal{T}_{f}, q\right)=\operatorname{sat}(Q)$, pois $\mathcal{S C}\left(\mathcal{T}_{f}, p\right), \mathcal{S C}\left(\mathcal{T}_{f}, q\right) \in \mathcal{S} \mathcal{A} \mathcal{T}_{\mathcal{U}}(f)$. Então, 
seguindo a definição do operador de saturação temos:

$$
\mathcal{S C}\left(\mathcal{T}_{f}, p\right)=\operatorname{sat}(P)=P \cup \bigcup_{H \in \operatorname{Int}(P)} H
$$

Assim, se $h \in H \in \operatorname{Int}(P)$, então $\mathcal{S C}\left(\mathcal{T}_{f}, h\right) \subset \mathcal{S C}\left(\mathcal{T}_{f}, p\right)$ e consequentemente $\mathcal{S C}\left(\mathcal{T}_{f}, p\right)$ não é a menor forma contendo o pixel $h$. Logo, como $\mathcal{S C}\left(\mathcal{T}_{f}, p\right)$ é a menor forma contendo $p$, então $p \notin \mathcal{S C}\left(\mathcal{T}_{f}, h\right)$. Portanto, $p \in P$ e analogamente $q \in Q$.

Agora, queremos mostrar que $p \in Q$ e assim concluir que $P \subset Q$, pois $(p, q) \in \mathcal{A}$ e $P$ e $Q$ são CCs de $\mathcal{U}(f)$. Então, suponha por contradição que $p \notin Q$. Logo, temos que $Q \subset P$ pelos mesmos argumentos anteriores. Então, como sat é um operador crescente (ver Lema 2.8 em Caselles et al. (2008)), segue que, se $Q \subseteq P$ então $\operatorname{sat}(Q) \subseteq \operatorname{sat}(P)$ o que é uma contradição. Portanto, $p \in Q$ e consequentemente $P \subset Q$. Portanto, graças à Proposição 5.1, segue que $f(p)>f(q)$.

Proposição 5.6. Seja $A \in \mathcal{L}(f) \cup \mathcal{U}(f)$ tal que sat $(A) \in \mathcal{S} \mathcal{A T}(f)$. Se $B \in \operatorname{Int}(A)$ e $(p, q) \in \mathcal{A}$ tal que $p \in B$ e $q \notin B$, então $q \in \operatorname{sat}(A)$ (Veja uma ilustração do enunciado na Figura 5.2).
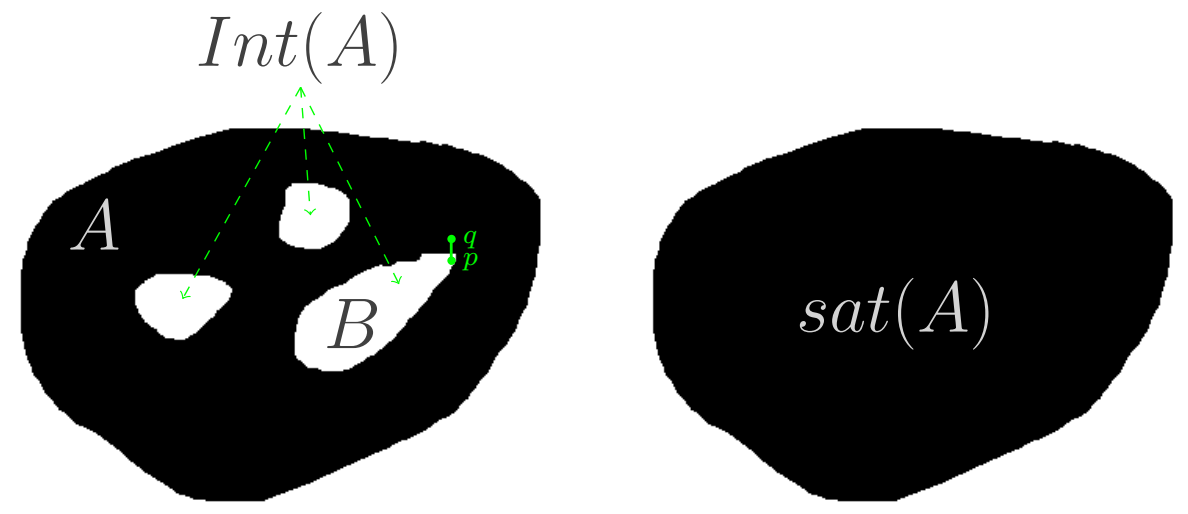

Figura 5.2: Ilustração do enunciado da Proposição 5.6.

Prova: Vamos mostrar que $q \in \operatorname{sat}(A)$ por contradição. Suponha, por contradição que $q \notin \operatorname{sat}(A)$.

Como $q \notin \operatorname{sat}(A)$ então $q$ pertence ao complemento de $\operatorname{sat}(A)$, ou seja, $q \in(\mathcal{D} \backslash \operatorname{sat}(A))=$ $\operatorname{Ext}(A) \subseteq(\mathcal{D} \backslash A)$. Como $\operatorname{Int}(A)$ contém os CCs de $(\mathcal{D} \backslash A)$ incluídas em sat $(A)$ e $B \in \operatorname{Int}(A)$, então temos que ambos $B$ e $\operatorname{Ext}(A)$ são $\operatorname{CCs}$ de $(\mathcal{D} \backslash A)$. Com este resultado em mente e pelo fato que $(p, q) \in \mathcal{A}$ com $p \in B$ e $q \in \operatorname{Ext}(A)$ conclui-se que $\operatorname{Ext}(A)=B$. Mas isso é uma contradição, pois $q \notin B$. Portanto, $q \in \operatorname{sat}(A)$.

O Corolário 5.7 a seguir é uma consequência direta da Proposição 5.6.

Corolário 5.7. Sejam $A, B \in \mathcal{S} \mathcal{A} \mathcal{T}(f)$ tal que $B \subset A, A \in \mathcal{S} \mathcal{A} \mathcal{T}_{\mathcal{U}}(f)$ e $B \in \mathcal{S} \mathcal{A} \mathcal{T}_{\mathcal{L}}(f)$ (respectivamente, $A \in \mathcal{S A T}_{\mathcal{L}}(f)$ e $\left.B \in \mathcal{S A}_{\mathcal{T}}(f)\right)$. Se $(p, q) \in \mathcal{A}$ tal que $p \in B$ e $q \notin B$, então ou $q \in A$, ou $\exists B^{\prime} \in \mathcal{S} \mathcal{A} \mathcal{T}_{\mathcal{U}}(f)$ (respectivamente, $\exists B^{\prime} \in \mathcal{S A}_{\mathcal{T}} \mathcal{T}_{\mathcal{L}}(f)$ ) tal que $B \subseteq B^{\prime} \subseteq A$ e $q \in B^{\prime}$

Proposição 5.8. Sejam $(p, q) \in \mathcal{A}, \mathcal{S C}\left(\mathcal{T}_{f}, p\right)$ e $\mathcal{S C}\left(\mathcal{T}_{f}, q\right)$ duas menores formas de $\mathcal{S} \mathcal{A} \mathcal{T}_{\mathcal{U}}(f)$ (respectivamente, $\mathcal{S} \mathcal{A} \mathcal{T}_{\mathcal{L}}(f)$ ) contendo os pixels p e q. Se $X \in \mathcal{S} \mathcal{A} \mathcal{T}(f)$ tal que $\mathcal{S C}\left(\mathcal{T}_{f}, p\right) \subset X \subset$ $\mathcal{S C}\left(\mathcal{T}_{f}, q\right)$, então $X \in \mathcal{S} \mathcal{A} \mathcal{T}_{\mathcal{U}}(f)$ (respectivamente, $X \in \mathcal{S} \mathcal{A} \mathcal{T}_{\mathcal{L}}(f)$ ). 
Prova: Suponha, por contradição, que $X \notin \mathcal{S} \mathcal{A} \mathcal{T}_{\mathcal{U}}(f)$. Assim, $X \in \mathcal{S} \mathcal{A} \mathcal{T}_{\mathcal{L}}(f)$ pois $X \in \mathcal{S} \mathcal{A} \mathcal{T}(f)$. Então, graças ao Corolário 5.7, segue que $q \in X$, pois $(p, q) \in \mathcal{A}, \mathcal{S C}\left(\mathcal{T}_{f}, p\right) \in \mathcal{S A T}_{\mathcal{U}}(f), X \in$ $\mathcal{S} \mathcal{A} \mathcal{T}_{\mathcal{L}}(f)$ e $\mathcal{S C}\left(\mathcal{T}_{f}, p\right) \subset X$. Mas isso é uma contradição, pois $\mathcal{S C}\left(\mathcal{T}_{f}, q\right)$ é a menor forma contendo q. Portanto, $X \in \mathcal{S} \mathcal{A} \mathcal{T}_{\mathcal{U}}(f)$.

Teorema 5.9. Reconstruções por formas são levelings.

Prova: Seja $f \in \mathcal{F}(\mathcal{D})$ uma imagem e $\mathcal{T}_{f}$ a árvore de formas construída a partir da imagem $f$. Então, $g \in \mathcal{F}(\mathcal{D})$ é uma reconstrução por formas de $f$ se, e somente se, $g=\operatorname{Rec}\left(\mathcal{T}_{g}\right)$ tal que $\mathcal{T}_{g}=\mathcal{P} \operatorname{oda}\left(\mathcal{T}_{f}\right)$. Para provar que $g$ é um leveling de $f$, é preciso verificar se para todo $(p, q) \in \mathcal{A}$, a definição de leveling é satisfeita, isto é, que $g(p)>g(q) \Rightarrow f(p) \geq g(p)$ e $g(q) \geq f(q)$.

Inicialmente, considere dois casos, quando $f(p)=f(q)$ e $f(p) \neq f(q)$. No primeiro caso, $g$ satisfaz a definição de levelings por vacuidade, pois $(p, q)$ pertence a uma mesma zona plana de $f$ e consequentemente no mesmo vértice de $\mathcal{T}_{f}$ e portanto $g(p)=g(q)$. No segundo caso, temos pelo Teorema 4.16, que duas formas $\mathcal{S C}\left(\mathcal{T}_{f}, p\right)$ e $\mathcal{S C}\left(\mathcal{T}_{f}, q\right)$ são disjuntas ou comparáveis. Logo, na operação de poda que gera $\mathcal{T}_{g}$ a partir de $\mathcal{T}_{f}$ pode-se: (1) ou preservar ambos os vértices; (2) ou eliminar ambos os vértices; (3) ou eliminar um dos dois vértices. Veja na Figura 5.3 as ilustrações sobre as configurações de podas.

Casos 1 e 2: triviais

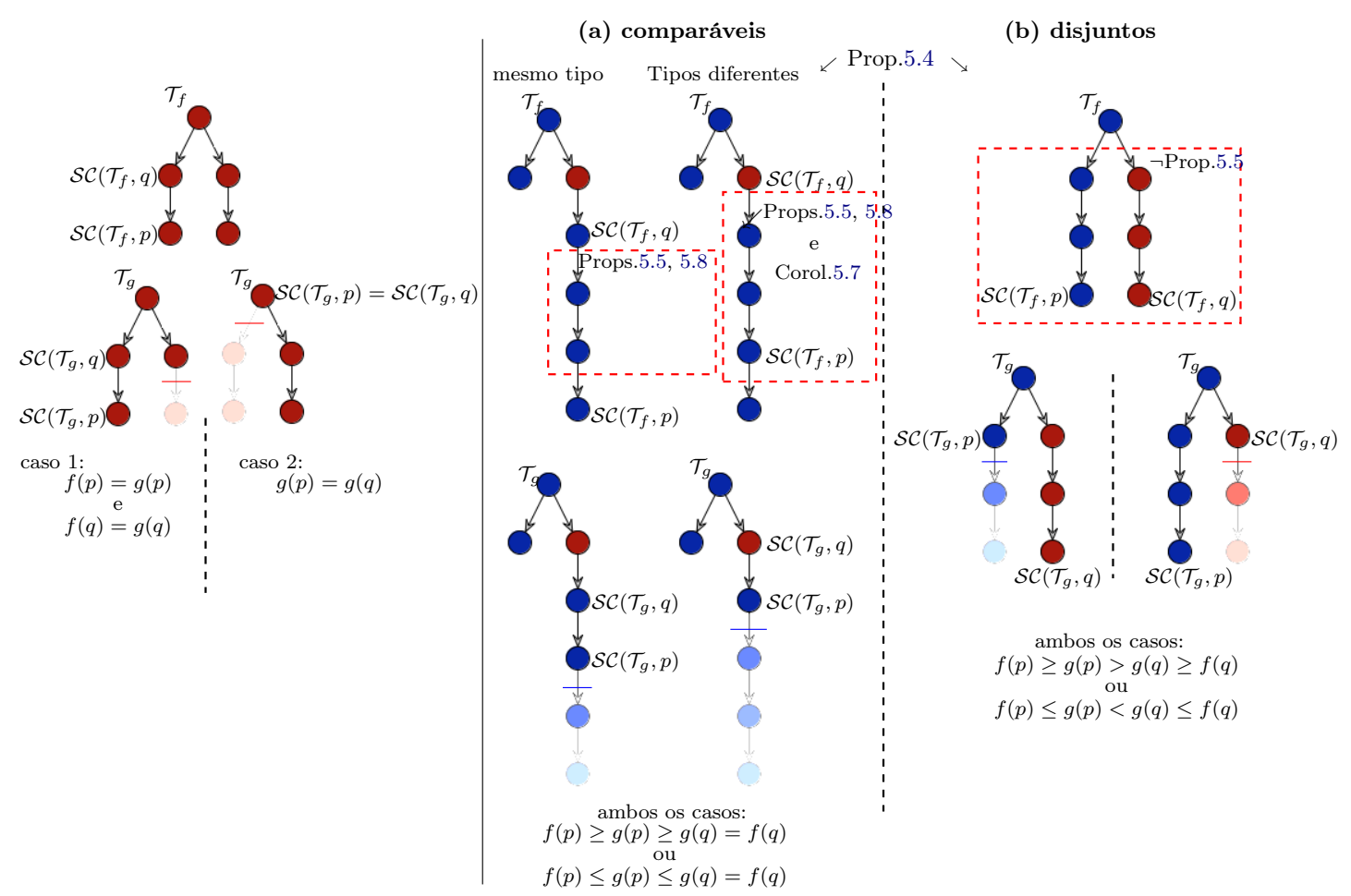

Figura 5.3: Ilustrações das configurações de podas.

1. Se ambos os vértices são preservados, então $f(p)=g(p)$ e $f(q)=g(q)$ o que satisfaz a condição da definição de leveling;

2. Se ambos os vértices são eliminados, então $g(p)=g(q)$ caso eles sejam comparáveis e assim a definição de leveling é verdadeira por vacuidade, caso contrário, veja o caso 3(b); 
3. Se um dos dois vértices é eliminado, então graças à Proposição $5.4, \mathcal{S C}\left(\mathcal{T}_{f}, p\right)$ e $\mathcal{S C}\left(\mathcal{T}_{f}, q\right)$ são disjuntas ou comparáveis.

(a) $\operatorname{Se~} \mathcal{S C}\left(\mathcal{T}_{f}, p\right)$ e $\mathcal{S C}\left(\mathcal{T}_{f}, q\right)$ são comparáveis, então sem perda de generalidade, suponha que $\mathcal{S C}\left(\mathcal{T}_{f}, p\right) \subset \mathcal{S C}\left(\mathcal{T}_{f}, q\right)$. Assim, $\mathcal{S C}\left(\mathcal{T}_{f}, p\right) \subseteq \mathcal{S C}\left(\mathcal{T}_{g}, p\right)$ e $\mathcal{S C}\left(\mathcal{T}_{f}, q\right)=\mathcal{S C}\left(\mathcal{T}_{g}, q\right)$ e consequentemente $g(q)=f(q)$.

- Se $\mathcal{S C}\left(\mathcal{T}_{f}, p\right)$ e $\mathcal{S C}\left(\mathcal{T}_{f}, q\right)$ pertencem $\mathcal{S} \mathcal{A} \mathcal{T}_{\mathcal{U}}(f)$ (respectivamente, $\mathcal{S} \mathcal{A} \mathcal{T}_{\mathcal{L}}(f)$ ) então graças à Proposição 5.8, segue que $\mathcal{S C}\left(\mathcal{T}_{g}, p\right) \in \mathcal{S} \mathcal{A} \mathcal{T}_{\mathcal{U}}(f)$. Assim, graças à Proposição 5.5 , segue que $f(p) \geq g(p) \geq g(q)=f(q)$ (respectivamente, $f(p) \leq g(p) \leq$ $g(q)=f(q))$ satisfazendo a condição da definição de leveling;

- Se $\mathcal{S C}\left(\mathcal{T}_{f}, p\right)$ e $\mathcal{S C}\left(\mathcal{T}_{f}, q\right)$ são de tipos diferentes, então graças ao Corolário 5.7, segue que $\forall(r, s) \in \mathcal{A}$ tal que $r \in \mathcal{S C}\left(\mathcal{T}_{f}, p\right)$ e $s \notin \mathcal{S C}\left(\mathcal{T}_{f}, p\right)$ segue que $s \in \mathcal{S C}\left(\mathcal{T}_{f}, q\right)$. Assim, $\mathcal{S C}\left(\mathcal{T}_{f}, p\right) \subset \mathcal{S C}\left(\mathcal{T}_{f}, s\right) \subseteq \mathcal{S C}\left(\mathcal{T}_{f}, q\right)$ e consequentemente ou $f(p)<f(s) \leq f(q) \Rightarrow$ $f(p) \leq g(p) \leq g(q)=f(q)$, ou $f(p)>f(s) \geq f(q) \Rightarrow f(p) \geq g(p) \geq g(q)=f(q)$, o que satisfaz a condição da definição de leveling.

(b) $\operatorname{Se~} \mathcal{S C}\left(\mathcal{T}_{f}, p\right)$ e $\mathcal{S C}\left(\mathcal{T}_{f}, q\right)$ são disjuntos, então eles são de tipos diferentes (ver Proposição 5.5). Além do mais, $\mathcal{S C}\left(\mathcal{T}_{f}, p\right)$ e $\mathcal{S C}\left(\mathcal{T}_{f}, q\right)$ não pertencem ao mesmo ramo em $\mathcal{T}_{f}$. Neste caso, certamente existe um vértice $\mathcal{S C}\left(\mathcal{T}_{f}, r\right)$ que é ancestral comum a ambos os vértices $\mathcal{S C}\left(\mathcal{T}_{f}, p\right)$ e $\mathcal{S C}\left(\mathcal{T}_{f}, q\right)$. Assim, $\mathcal{S C}\left(\mathcal{T}_{f}, r\right)$ é do mesmo tipo que $\mathcal{S C}\left(\mathcal{T}_{f}, p\right)$ ou $\mathcal{S C}\left(\mathcal{T}_{f}, q\right)$. Então, ou $f(p)>f(r)>f(q)$, ou $f(p)<f(r)<f(q)$. Sem perda de generalidade, assume-se que $f(p)>f(r)>f(q)$. Dessa forma, se $\mathcal{S C}\left(\mathcal{T}_{f}, p\right)$ é removido e $\mathcal{S C}\left(\mathcal{T}_{f}, q\right)$ é preservado, então temos que $\mathcal{S C}\left(\mathcal{T}_{f}, p\right) \subset \mathcal{S C}\left(\mathcal{T}_{g}, p\right) \subseteq \mathcal{S C}\left(\mathcal{T}_{f}, r\right)$ e $\mathcal{S C}\left(\mathcal{T}_{g}, q\right)=\mathcal{S C}\left(\mathcal{T}_{f}, q\right)$ e consequentemente $f(p)>g(p) \geq f(r)$ e $g(q)=f(q)$. Portanto, $f(p)>g(p) \geq f(r)>g(q)=f(q) \Rightarrow f(p)>g(p)>g(q)=f(q)$, satisfazendo a condição da definição de leveling. Mas, se $\mathcal{S C}\left(\mathcal{T}_{f}, p\right)$ é preservado e $\mathcal{S C}\left(\mathcal{T}_{f}, q\right)$ é removido, então temos que $\mathcal{S C}\left(\mathcal{T}_{g}, p\right)=\mathcal{S C}\left(\mathcal{T}_{f}, p\right)$ e $\mathcal{S C}\left(\mathcal{T}_{f}, q\right) \subset \mathcal{S C}\left(\mathcal{T}_{g}, q\right) \subseteq \mathcal{S C}\left(\mathcal{T}_{f}, r\right)$ e consequentemente $f(p)=g(p)$ e $f(r) \geq g(q)>f(q)$. Portanto, $f(p)=g(p)>g(q)>f(q)$, o que satisfaz a condição da definição de leveling.

\subsection{Operadores morfológicos obtidos por meio de reconstruções de árvores po- dadas}

Nesta seção são estabelecidas relações de diversos operadores conhecidos na literatura de MM com reconstruções de árvores podadas e por consequência, com os levelings. Pode-se citar, por exemplo: reconstrução por dilatação e erosão, abertura e fechamento por atributos, filtros por grãos, entre outros. Basicamente, na construção destes operadores cada vértice passa por uma decisão que opta em preservar ou eliminar o vértice e esta decisão pode ser classificada em duas categorias: (1) baseada em uma imagem marcadora ou (2) baseada no valor de um atributo crescente.

\subsubsection{Operadores baseados em imagens marcadoras}

Os levelings são operadores intimamente relacionados com operadores baseados em imagens marcadoras, como mostrado na Seção 3.5. Conforme apresentado, na Seção 2.2 uma imagem binária $\mathcal{X} \in \mathcal{P}(\mathcal{D})$ pode ser reconstruída a partir de uma imagem marcadora $\mathcal{M} \subseteq \mathcal{X}$ por meio do operador 
dilatação condicional $\delta_{\mathcal{B}}^{n}$ até atingir a idempotência e quando atinge, chama-se de reconstrução por dilatação $\delta_{\mathcal{B}}^{\infty}$. Este operador, também pode ser definido simplesmente pela união dos CCs do conjunto $\mathcal{X}$ que contém pelo menos um pixel do marcador $\mathcal{M}$ (Vincent, 1993), isto é, $\delta_{\mathcal{B}}^{\infty}(\mathcal{X}, \mathcal{M})=$ $\left\{C \in \mathcal{C C}\left(X, \mathcal{A}_{\mathcal{B}}\right): C \cap \mathcal{M} \neq \emptyset\right\}$. Assim, como é conhecido há diversas décadas, qualquer operador crescente, como a reconstrução por dilatação, definido para imagens binárias pode ser estendido para imagens em níveis de cinza por meio de decomposição por limiarização (Maragos e Ziff, 1990; Wendt et al., 1986). Logo, aplicando a decomposição por limiarização em uma dada imagem $f \in$ $\mathcal{F}(\mathcal{D})$ e uma imagem marcadora $g \in \mathcal{F}(\mathcal{D})$ tal que $g \leq f$, temos que:

$$
\begin{aligned}
& \forall p \in \mathcal{D},\left[\delta_{\mathcal{B}}^{\infty}(f, g)\right](p)=\sup \left\{\lambda \in \mathbb{K}: p \in \delta_{\mathcal{B}}^{\infty}\left(\mathcal{X}_{\lambda}^{\uparrow}(f), \mathcal{X}_{\lambda}^{\uparrow}(g)\right)\right\} \\
& \Longleftrightarrow\left[\delta_{\mathcal{B}}^{\infty}(f, g)\right](p)=\sup \left\{\lambda \in \mathbb{K}: p \in\left\{C \in \mathcal{C} \mathcal{C}\left(\mathcal{X}_{\lambda}^{\uparrow}(f), \mathcal{A}_{\mathcal{B}}\right) \text { tal que } C \cap \mathcal{X}_{\lambda}^{\uparrow}(g) \neq \emptyset\right\}\right\} \\
& \Longleftrightarrow\left[\delta_{\mathcal{B}}^{\infty}(f, g)\right](p)=\sup \left\{\lambda \in \mathbb{K}: p \in C \cap \mathcal{X}_{\lambda}^{\uparrow}(g) \neq \emptyset \text { tal que }(C, \lambda) \in \operatorname{Ext}(\mathcal{U}(f))\right\} .
\end{aligned}
$$

Note que, para construir $\delta_{\mathcal{B}}^{\infty}(f, g)$ para um pixel $p \in \mathcal{D}$, é preciso encontrar o menor conjunto $\mathcal{X}_{\lambda}^{\uparrow}(g)$ tal que $\mathcal{X}_{\lambda}^{\uparrow}(g) \cap C \neq \emptyset$ e isto pode ser expresso como uma operação de poda na versão estendida da max-tree $(\mathcal{U}(f), \subseteq)$, por meio do seguinte procedimento: na construção de $\delta_{\mathcal{B}}^{\infty}(f, g)$ cada vértice $(C, \mu)$ de $(\operatorname{Ext}(\mathcal{U}(f)), \sqsubseteq)$ passa por uma decisão que opta em eliminá-lo (respectivamente, preserválo) se, e somente se, existir um pixel $p \in C$ tal que $\mu>g(p)$ (respectivamente, $g(p) \geq \mu$ ), pois $\mu>g(p)$ implica em $\mathcal{X}_{\lambda}^{\uparrow}(g) \cap C \neq \emptyset$ e como pode ser observado, por exemplo, nas Figuras 5.4 e 5.5. Portanto,

$$
\delta_{\mathcal{B}}^{\infty}(f, g)=\operatorname{Rec}\left(\left(\mathcal{T}_{\mathcal{U}}^{g}, \sqsubseteq\right)\right)
$$

tal que $\mathcal{T}_{\mathcal{U}}^{g}=\left\{(C, \mu) \in \operatorname{Ext}(\mathcal{U}(f)): \bigvee_{p \in C} g(p) \geq \mu\right\}$

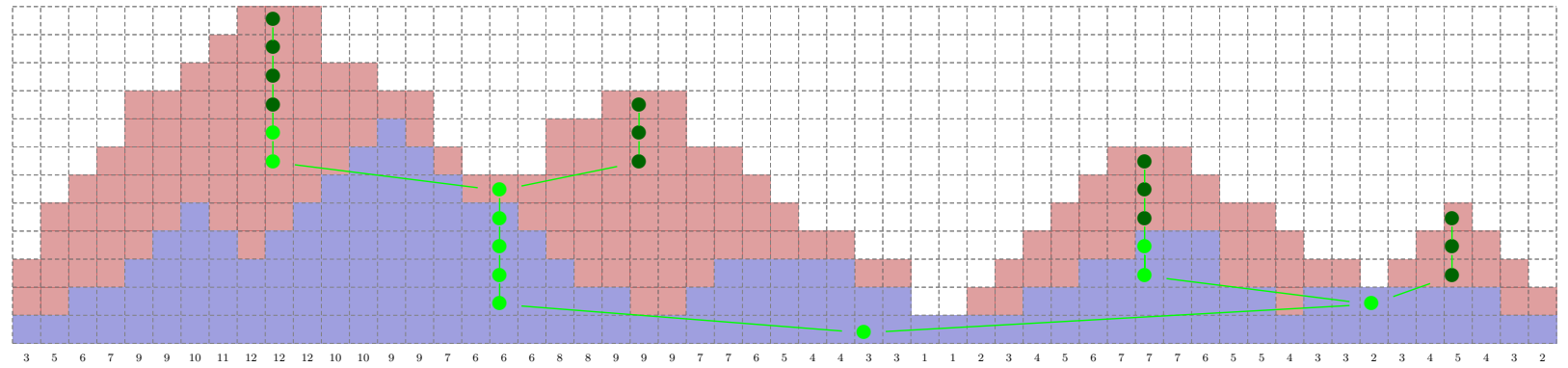

Figura 5.4: Ilustração destacando a imagem $f$ em vermelho, a imagem marcadora $g$ em azul e a versão estendida da max-tree $(\operatorname{Ext}(\mathcal{U}(f)), \sqsubseteq)$ em verde. Note que, a imagem g está sobreposta a imagem $f$ e os vértices em verde escuro não estão presentes no conjunto $\mathcal{T}_{\mathcal{U}}^{g}$.

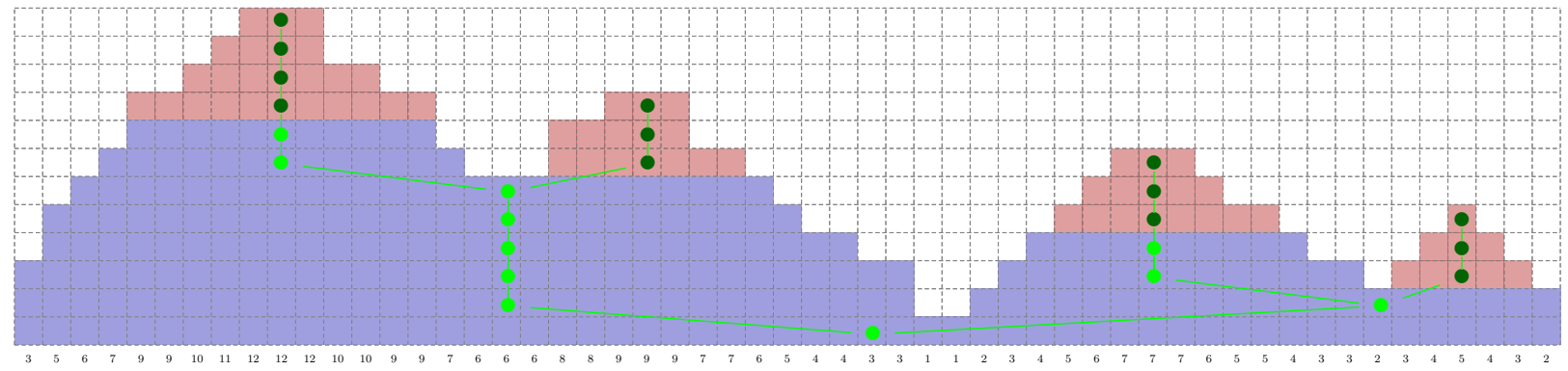

Figura 5.5: Ilustração destacando a imagem $f$ em vermelho, a imagem resultante da reconstrução por dilatação $\delta_{\mathcal{B}}^{\infty}(f, g)$ em azul e a versão estendida da max-tree $(\operatorname{Ext}(\mathcal{U}(f)), \sqsubseteq)$ em verde.

Seguindo estas ideias, é proposto o Algoritmo 5.1 (semelhante ao algoritmo de Wilkinson et al. (2011)) para computar a reconstrução por dilatação. Este algoritmo, inicialmente marca todos os vértices da max-tree estendida $\mathcal{T}_{f}$ para serem podados, depois faz uso de uma fila de prioridade $Q$ para desmarcar os vértices que não serão podados. Dessa forma, primeiramente todos os pixels são 
adicionados em $Q$ (linhas 5 e 6) e assim, quando um pixel $p$ é retirado da fila de prioridade $Q$ para ser processado (linhas 8 e 9), é encontrado o menor componente $\mathcal{S C}\left(\mathcal{T}_{f}, p\right)$ contendo este pixel p. Então (linhas 10 a 15$)$, se o vértice $\mathcal{S C}\left(\mathcal{T}_{f}, p\right)$ não foi processado, é encontrado o primeiro ancestral $(C, \mu)$ de $\mathcal{S C}\left(\mathcal{T}_{f}, p\right)$ tal que $\mu \geq g(p)$ e assim todo o intervalo partindo deste vértice $(C, \mu)$ até a raiz da árvore (ou melhor ainda, até o primeiro vértice já desmarcado) é desmarcado, ou seja, não serão podados. Após processar todos os pixels de $\mathcal{D}$, temos marcados quais vértices serão podados de $\mathcal{T}_{f}$ para construir a versão podada na qual sua reconstrução coincide com a reconstrução por dilatação. Note que, o intervalo de vértice de $(C, \mu)$ até a raiz da árvore é sempre menor, pois se existir no caminho um vértice já desmarcado então não é necessário reprocessá-lo. Por fim, na linha 16 é reconstruída a imagem da árvore podada.

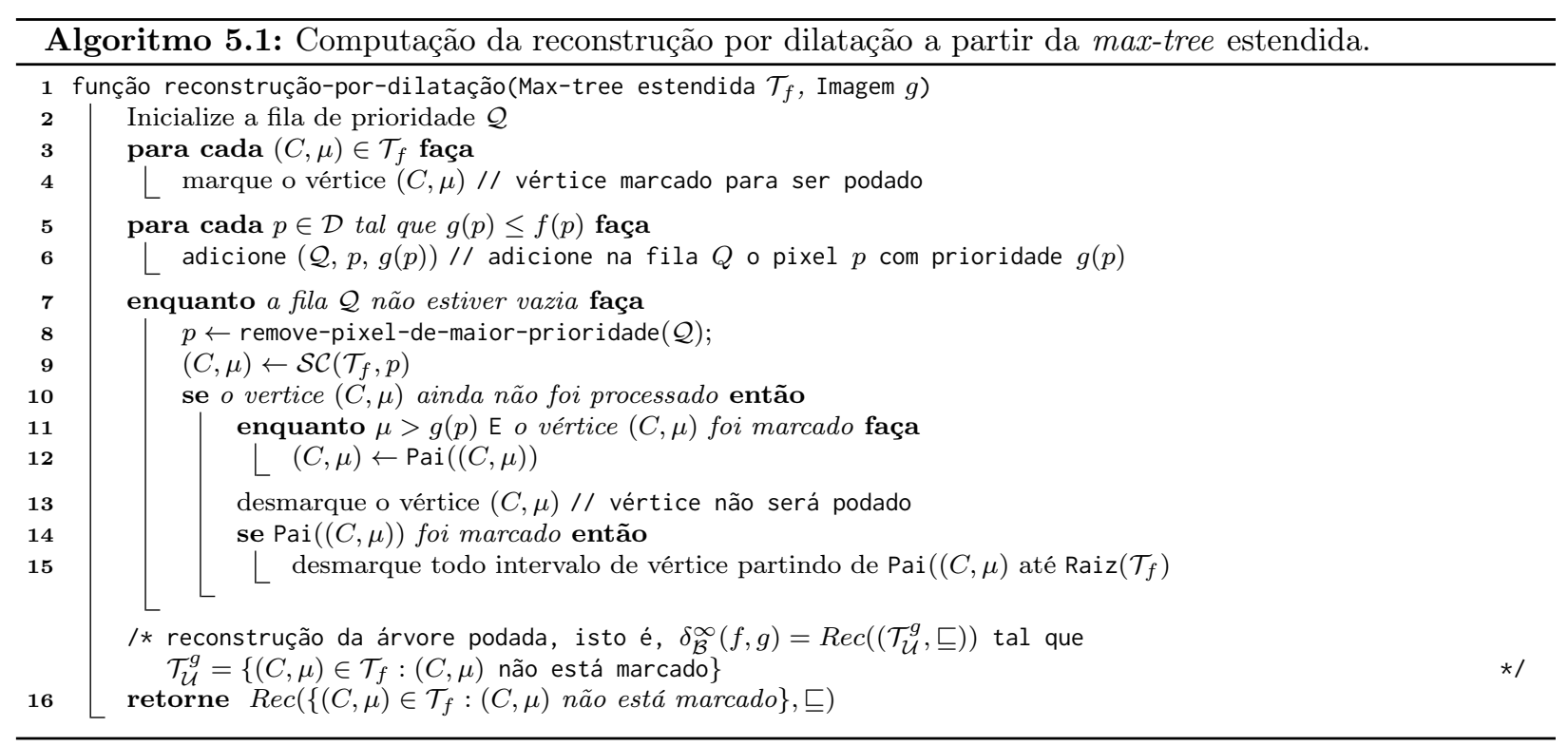

O Algoritmo 5.1 é rápido e sua complexidade computacional é linear na quantidade de pixels. Isso pode ser visto nos gráficos apresentados nas Figuras 5.6 e 5.7 comparando os tempos computacionais do Algoritmo 5.1 e dos algoritmos propostos por Vincent (1993) e Falcao et al. (2001), para computação da reconstrução por dilatação usando um computador com processador $i 7$ e $8 \mathrm{~GB}$ de memória RAM.

Assim, por dualidade pode-se ter a reconstrução por erosão ou similarmente pode ser definida ainda usando os conjuntos de níveis inferiores. Então, dado uma imagem $f \in \mathcal{F}(\mathcal{D})$ e um imagem marcadora $g \in \mathcal{F}(\mathcal{D})$ tal que $g \geq f$, temos:

$$
\varepsilon_{\mathcal{B}}^{\infty}(f, g)=\operatorname{Rec}\left(\left(\mathcal{T}_{\mathcal{L}}^{g}, \sqsubseteq\right)\right)
$$

tal que $\mathcal{T}_{\mathcal{L}}^{g}=\left\{(C, \mu) \in \mathcal{T}_{f}: \bigwedge_{p \in C} g(p) \leq \mu\right\}$

As reconstruções por erosão e dilatação dão origem a um grande número de operadores morfológicos, tais como: abertura e fechamento por reconstrução, top-hat por reconstrução, $h$-bacia, $h$-domos, entre outros (Dougherty e Lotufo, 2003; Soille, 2003; Vincent, 1993). Além disso, seguindo Meyer (1998b), pode-se definir o operador auto-dual self-reconstrução combinando a reconstrução por dilatação com a reconstrução por erosão. Neste caso, ambas as árvores precisam ser definidas com a 


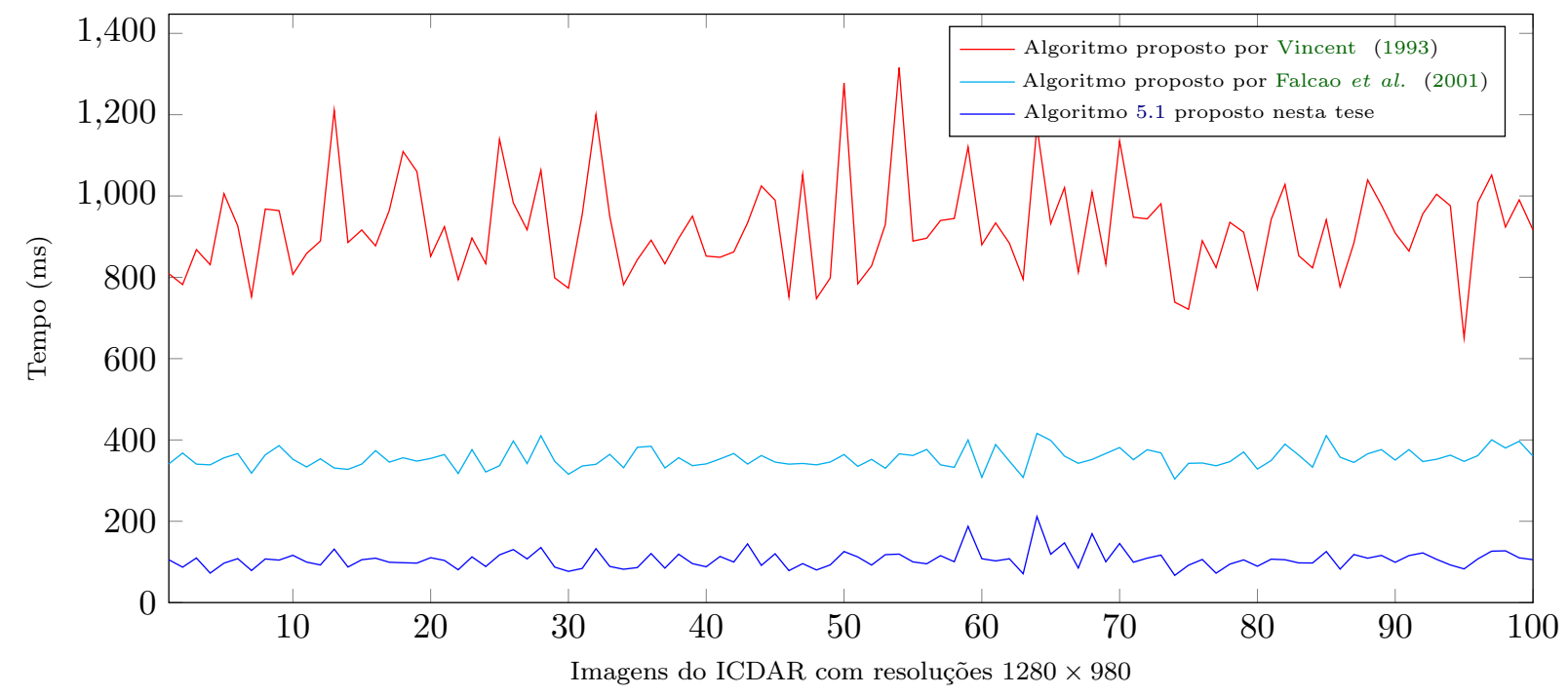

Figura 5.6: Comparação dos tempos computacionais do Algoritmo 5.1 e dos algoritmos propostos por Vincent (1993) e Falcao et al. (2001). Nesta comparação, cada imagem do dataset ICDAR (Lucas et al., 2003) é avaliada com 10 diferentes imagens marcadoras e a média destas avaliações é plotada neste gráfico.

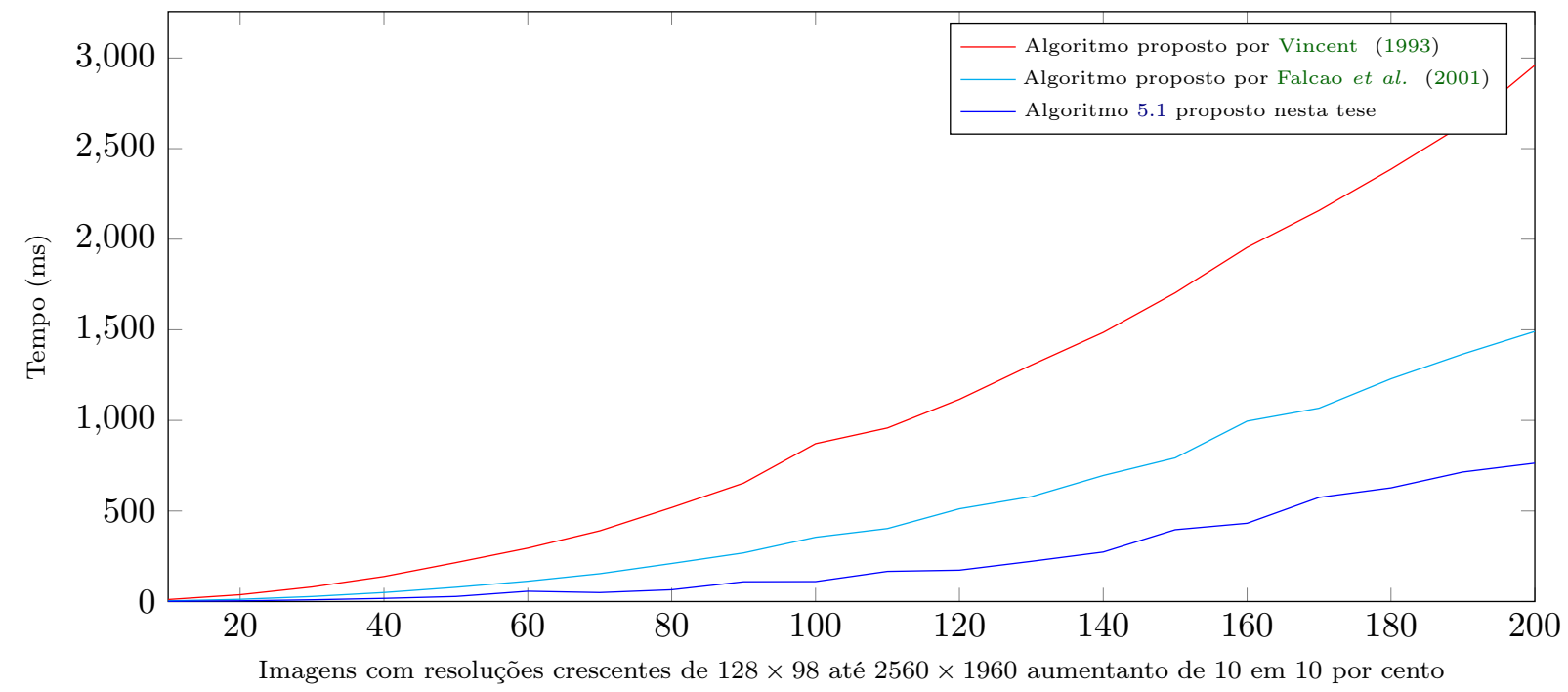

Figura 5.7: Comparação dos tempos computacionais do Algoritmo 5.1 e dos algoritmos propostos por Vincent (1993) e Falcao et al. (2001). Nesta comparação, cada imagem do dataset ICDAR (Lucas et al., 2003) é avaliada com 10 diferentes imagens marcadoras e a média destas avaliações é plotada neste gráfico para cada resolução analisada (de $128 \times 98$ até $2560 \times 1960$ aumentando de 10 em 10 por cento).

mesma relação de adjacência.

$$
p \in \mathcal{D},[\rho(f, g)](p)= \begin{cases}\delta_{\mathcal{B}}^{\infty}(f, g \wedge f), & \text { se } g(p)<f(p), \\ \varepsilon_{\mathcal{B}}^{\infty}(f, g \vee f), & \text { se } g(p)>f(p), \\ f(p), & \text { caso contrário. }\end{cases}
$$

Como verificado na Seção 5.1 estes operadores baseados em reconstrução da árvore estendida max-tree e min-tree são todos levelings e pela Proposição 5.10 proposta por Meyer (1998b) garante que o operador self-reconstrução também é leveling. 
Proposição 5.10 (Meyer (1998b)). O operador $\rho(f, g)$ é um leveling da imagem $f \in \mathcal{F}(\mathcal{D})$, para qualquer imagem marcadora $g \in \mathcal{F}(\mathcal{D})$.

\subsubsection{Operadores baseados em atributos}

Tradicionalmente, a árvore de componentes e a árvore de formas são utilizadas para construir operadores conexos por meio de uma operação de podas ou eliminação de vértices da árvore segundo algum critério sobre atributos extraídos dos vértices. Neste sentido, cada vértice da árvore passa por uma decisão que resulta em preservar ou eliminar o vértice de acordo com o critério de decisão sobre atributos extraídos dos vértices. Assim, como vértices representam CCs dos conjuntos de níveis, então pode-se extrair diversos atributos (e representá-los por meio de uma função $\kappa: \mathcal{P}(\mathcal{D}) \rightarrow \mathbb{R}$ ) destes CCs, como por exemplo: área, volume, altura e largura do retângulo envolvente sobre o CC, perímetro, circularidade, momentos, entre outros. Estes atributos podem ainda ser classificados como crescentes e não-crescentes. Dessa forma, um atributo $\kappa$ é crescente se, e somente se, para quais dois vértices $A$ e $B$ tal que $A \subseteq B$ implica que $\kappa(A) \leq \kappa(B)$, caso contrário, o atributo $\kappa$ é não-crescente. Por exemplo a área, volume, altitude, altura e largura da caixa envolvente são exemplos de atributos crescentes extraídos de um vértice. Enquanto que o perímetro, momentos, circularidade, atributos de textura são exemplos de atributos não-crescentes (Salembier e Wilkinson, 2009; Salembier et al., 1998; Urbach e Wilkinson, 2002; Urbach et al., 2004; Xu, 2013).

Se os atributos extraídos forem crescentes, os valores dos atributos também seguirão uma ordem bem definida na árvore devido à hierarquia de inclusão dos conjuntos de níveis. Graças a esta propriedade, pode-se obter os operadores conexos, abertura por atributo, fechamento por atributo e filtro por grão, simplesmente podando os vértices da max-tree, min-tree e árvore de formas, respectivamente, cujo valor do atributo crescente $\kappa$ é menor que um dado limiar $\alpha$, seguido da reconstrução da árvore podada (Caselles e Monasse, 2002; Salembier et al., 1998).

Dessa forma, define-se a abertura por atributo $\gamma_{\alpha}^{\kappa}$, o fechamento por atributo $\phi_{\alpha}^{\kappa}$ e o filtro por grão $\nu_{\alpha}^{\kappa}$ para toda imagem $f \in \mathcal{F}(\mathcal{D})$ por meio de reconstruções das árvores podadas max-tree $(\mathcal{U}(f), \subseteq)$, min-tree $(\mathcal{L}(f), \subseteq)$ e árvore de formas $(\mathcal{S} \mathcal{A} \mathcal{T}(f), \subseteq)$, da seguinte forma:

$$
\begin{gathered}
\gamma_{\alpha}^{\kappa}(f)=\operatorname{Rec}(\{C \in \mathcal{L}(f): \kappa(C)>\alpha\}, \subseteq), \\
\phi_{\alpha}^{\kappa}(f)=\operatorname{Rec}(\{C \in \mathcal{U}(f): \kappa(C)>\alpha\}, \subseteq) \mathrm{e} \\
\nu_{\alpha}^{\kappa}(f)=\operatorname{Rec}(\{C \in \mathcal{S} \mathcal{A} \mathcal{T}(f): \kappa(C)>\alpha\}, \subseteq) .
\end{gathered}
$$

De uma forma geral, a filtragem utilizando atributo crescente é algo simples, ou seja, descarta os vértices que não satisfazem o critério e reconstrói a imagem com os vértices restantes. Para os atributos não-crescentes, a decisão sobre a remoção dos vértices é menos direta. Exemplos de estratégias para eliminação de vértice (o que não satisfaz necessariamente a Definição 4.10 de poda) utilizando atributos não-crescentes podem ser encontrados em Salembier et al. (1998) e Urbach e Wilkinson (2002), onde são apresentadas três estratégias de podas:

- Min: um vértice $N_{k}$ é removido, ou se $\kappa\left(N_{k}\right)<\alpha$, ou se existe um vértice ascendente $N_{a}$, tal que $\kappa\left(N_{a}\right)<\alpha$.

- Max: um vértice $N_{k}$ é removido, se $\kappa\left(N_{k}\right)<\alpha$ e todos os seus descendentes $N_{d}$ satisfazem $\kappa\left(N_{d}\right)<\alpha$. 
- Viterbi: As remoções ou preservações dos vértices são determinadas por um processo de otimização de custos em um grafo. Neste sentido, cada vértice $N$ recebe dois estados, remoção e preservação, e quatro novas arestas que conectam estes estados, ou seja, os estados remoção e preservação de $N$ conectam (cada um) os estados de remoção e preservação de um outro vértice. Estas arestas recebem custos e o problema consiste em determinar os caminhos de menores custos saindo das folhas até a raiz. Assim, são removidos os vértices com estado de remoção destes caminhos de menores custos.

Vale salientar que operadores conexos obtidos por atributos não-crescentes não necessariamente são levelings. Nesta tese, temos interesse somente em operadores obtidos por podas, ou seja, por meio de atributos crescentes.

\subsection{Espaço de escala baseado em levelings por meio de podas sucessivas}

Como já conhecido por diversos anos que operadores conexos podem ser obtidos por reconstrução de árvore podada (Caselles e Monasse, 2002; Salembier et al., 1998), como por exemplo: a abertura (respectivamente, fechamento) por atributo e filtro por grãos. A partir da Seção 5.2.1, mostrou-se que operadores baseados em imagens marcadoras também podem ser obtidos por meio de podas nas versões estendidas da max-tree e min-tree, como por exemplo: reconstrução por dilatação, reconstrução por erosão, self-reconstrução, entre outros. Aproveitando-se dessa propriedade, nesta seção é mostrada uma maneira de construir uma representação de espaço de escala por meio de uma sequência de podas sucessivas.

Definição 5.11 (Sequência de podas sucessivas). Seja $\mathcal{T}_{f}$ a árvore (max-tree, min-tree ou árvore de formas) construída a partir de uma dada imagem $f \in \mathcal{F}(\mathcal{D})$, a sequência de podas sucessivas da árvore $\mathcal{T}_{f}$ é dada pela seguinte relação de recorrência:

$$
\mathcal{T}_{f}^{i}= \begin{cases}\mathcal{T}_{f} & , \text { se } i=0 \\ \mathcal{P} \operatorname{oda}\left(\mathcal{T}_{f}^{i-1}\right) & , \text { se } i>0\end{cases}
$$

para $i=0,1,2, \ldots$

Considere $\mathcal{T}_{f}$ uma árvore (max-tree, min-tree ou árvore de formas) construída a partir de uma dada imagem $f \in \mathcal{F}(\mathcal{D})$. Então, uma vez que os operadores levelings podem ser aninhados para criar um espaço de escala de uma imagem, como apresentado na Seção 3.6, e pelos Teoremas 5.3 e 5.9, temos que a sequência de reconstruções de podas sucessivas:

$$
\left(\psi_{0}(f), \psi_{1}(f), \ldots, \psi_{\mathcal{I}_{M A X}}(f)\right) \operatorname{com} \psi_{i}(f)=\operatorname{Rec}\left(\mathcal{T}_{f}^{i}\right), \text { para } i \in \mathcal{I}=\left\{0,1, \ldots, \mathcal{I}_{M A X}\right\}
$$

constitui um espaço de escala da imagem $f$ baseado em levelings e isto nos leva ao Teorema 5.12. Na Figura 5.8 é apresentado um exemplo simples de espaço de escala gerado por uma max-tree.

Teorema 5.12. A sequência de reconstruções de podas sucessivas de uma árvore (max-tree, min-tree ou árvore de formas) é um espaço de escala com base em levelings.

Prova: Considere $\left(\psi_{0}(f), \psi_{1}(f), \ldots, \psi_{\mathcal{I}_{M A X}}(f)\right)$ a sequência de reconstruções de podas sucessivas derivada da árvore $\mathcal{T}_{f}$ construída a partir de uma dada imagem $f \in \mathcal{F}(\mathcal{D})$, ou seja, para cada $i \in \mathcal{I}, \psi_{i}(f)=\operatorname{Rec}\left(\mathcal{T}_{f}^{i}\right)$. Assim, pela Definição 5.11 temos que a árvore $\mathcal{T}_{f}^{0}=\mathcal{T}_{f}$ e $\mathcal{T}_{f}^{i}$ é obtida por uma operação de poda da árvore $\mathcal{T}_{f}^{i-1}$ para $1 \leq i \leq \mathcal{I}_{M A X}$. Logo, graças aos Teoremas 5.3 e 


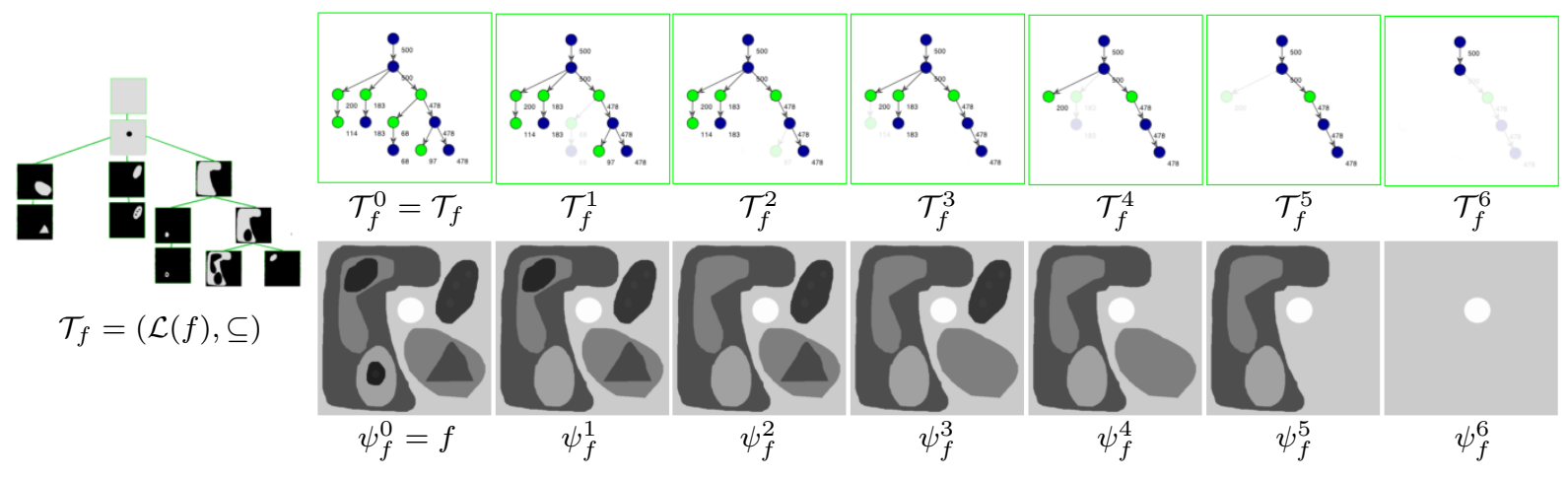

Figura 5.8: Exemplo de um espaço de escala gerado a partir de uma max-tree.

5.9, temos que $\psi_{i}(f)$ é um leveling de $\psi_{i-1}(f)$ e por transitividade temos que $\psi_{i}(f)$ é um leveling $\psi_{j}(f)$ para qualquer $0 \leq j \leq i \leq \mathcal{I}_{M A X}$ o que mostra que os levelings estão encadeados. Portanto, como apresentado na Seção 5.2.1, o encadeamento de levelings constitui um espaço de escala como mostrado em Meyer e Maragos (1999).

Com base nestas considerações, pode-se construir um espaço de escala a partir de uma árvore $\mathcal{T}_{f}$ por meio de reconstruções de podas sucessivas obtidas por uma estratégia baseada em atributos ou imagens marcadoras. Estes espaços de escalas são usados no Capítulo 6 para definir operadores da classe dos últimos levelings. Também, vale lembrar que muitos trabalhos sobre operadores residuais estendidos (Alves e Hashimoto, 2014; Alves et al., 2013; Beucher, 2007; Marcotegui et al., 2011; Meyer, 2010; Retornaz e Marcotegui, 2007), representam um espaço de escala por meio de uma família de operadores $\left\{\psi_{i}: i \in \mathcal{I}\right\}$ indexada pelo conjunto de índices $\mathcal{I}$. Assim, no caso de espaço de escala baseado em levelings, temos que para quaisquer $i, j \in \mathcal{I}, i \leq j \Leftrightarrow \psi_{j}$ é um leveling de $\psi_{i}$. Dessa forma, esta família de levelings indexada por $\mathcal{I}$ aplicada em uma imagem $f \in \mathcal{F}(\mathcal{D})$ dá origem ao espaço de escala $\left(\psi_{0}(f), \psi_{1}(f), \ldots, \psi_{\mathcal{I}_{M A X}}(f)\right)$.

\subsubsection{Espaço de escala baseado em atributos}

Um espaço de escala de aberturas (respectivamente, fechamentos) por atributo e filtros por grãos pode ser construído a partir de uma sequência crescente de limiares $\left(t_{1}, t_{2}, \ldots, t_{\mathcal{I}_{M A X}}\right)$ para um atributo crescente $\kappa$. Por exemplo, a sequência crescente de limiares pode ser derivada da própria árvore $\mathcal{T}_{f}$ da seguinte forma: $t_{i} \in T_{\kappa}=\left\{\kappa(A): A\right.$ é um vértice de $\left.\mathcal{T}_{f}\right\}$ tal que $t_{i-1}<t_{i}$ para $1<i \leq\left|T_{\kappa}\right|=\mathcal{I}_{M A X}$. Dessa forma, considerando $\mathcal{T}_{f}$ o poset $(\mathcal{V}, \subseteq)$, a sequência de podas sucessivas é dada por

$$
\mathcal{T}_{f}^{i}= \begin{cases}\mathcal{T}_{f} & , \text { se } i=0, \\ \mathcal{P} \operatorname{oda}\left(\mathcal{T}_{f}^{i-1}\right)=\left(\left\{A \in \mathcal{V}: \kappa(A)>t_{i}\right\}, \subseteq\right) & \text {, se } i>0,\end{cases}
$$

para $i=0,1, \ldots, \mathcal{I}_{M A X}$. Assim,

$$
\left(\psi_{0}(f), \psi_{1}(f), \ldots, \psi_{\mathcal{I}_{M A X}}(f)\right) \operatorname{com} \psi_{i}(f)=\operatorname{Rec}\left(\mathcal{T}_{f}^{i}\right), \text { para } i \in \mathcal{I}
$$

é um espaço de escala derivado de uma sequência crescente de limiares para um atributo $\kappa$. Na Figura 5.9 são apresentadas algumas imagens obtidas dos espaços de escalas baseados no atributo de área, usando as árvores max-tree, min-tree e de formas, respectivamente. 
max-tree - espaço de escala de aberturas por atributos
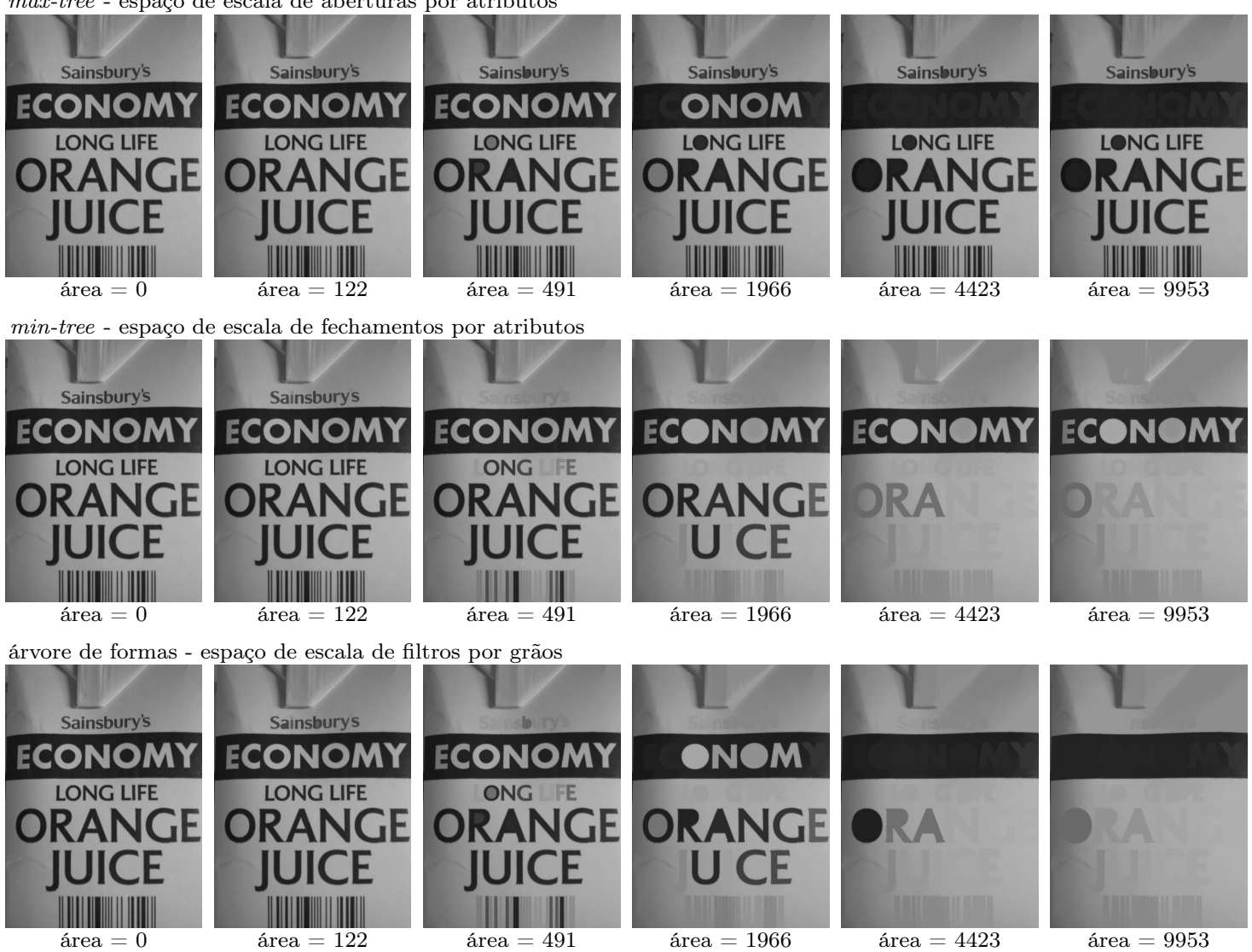

Figura 5.9: Exemplos de imagens de um espaço de escala de levelings obtidos por podas pelo atributo de área das árvores max-tree, min-tree e de formas.

\subsubsection{Espaço de escala baseado em imagens marcadoras}

Um espaço de escala de operadores baseados em reconstruções pode ser construído a partir de uma família de imagens marcadoras $\left(g_{1}, g_{2}, \ldots, g_{I_{M A X}}\right)$. Neste sentido, como mostra o trabalho de Meyer e Maragos (1999), diferentes famílias de marcadores podem ser utilizadas, como por exemplo, as seguintes famílias: extensivas de dilatações ou fechamentos, anti-extensivas de erosões ou aberturas, filtros sequenciais alternados, entre outras. Assim, se $\mathcal{T}_{f}$ é uma max-tree tal que a versão estendida de $\mathcal{T}_{f}$ é o poset $(\operatorname{Ext}(\mathcal{U}(f))$, $)$ então a sequência de podas sucessivas é dada por

$$
\mathcal{T}_{f}^{i}= \begin{cases}(\operatorname{Ext}(\mathcal{U}(f)), \sqsubseteq) & , \text { se } i=0, \\ \mathcal{P} o d a\left(\mathcal{T}_{f}^{i-1}\right)=\left(\left\{(C, \mu) \in \mathcal{T}_{f}^{i-1}: \bigvee_{p \in C} g_{i}(p) \geq \mu\right\}, \sqsubseteq\right) & , \text { se } i>0\end{cases}
$$

para $i=0,1, \ldots, \mathcal{I}_{M A X}$. Assim,

$$
\left(\psi_{0}(f), \psi_{1}(f), \ldots, \psi_{\mathcal{I}_{M A X}}(f)\right) \operatorname{com} \psi_{i}(f)=\operatorname{Rec}\left(\mathcal{T}_{f}^{i}\right), \text { para } i \in \mathcal{I}
$$

é um espaço de escala de levelings anti-extensivos derivado de uma família de imagens marcadoras. Analogamente, um espaço de escala de levelings extensivos pode ser construído por meio de uma min-tree. Também, pode-se construir um espaço de escala de levelings auto-duais combinando os resultados da reconstrução usando max-tree e, como definido na Equação 5.3. Na Figura 5.10 são apresentados alguns exemplos de imagens de espaços de escalas de levelings associados com famílias de marcadores. 
família de marcadores produziada por aberturas

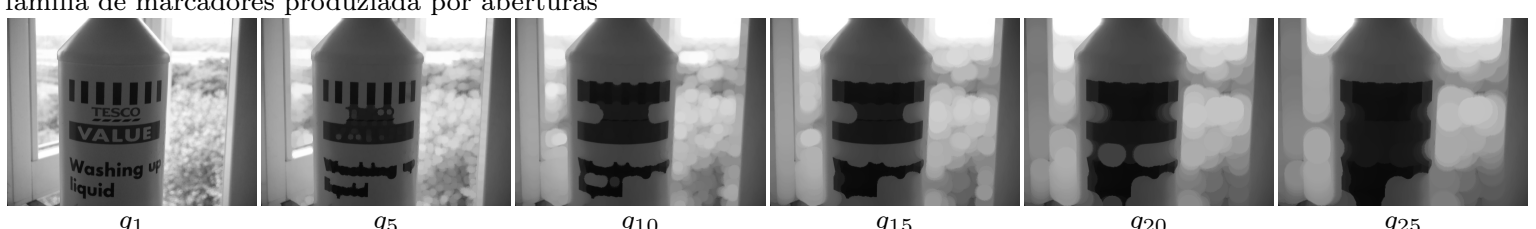

$g_{1}$

$g_{10}$

$g_{15}$

$g_{20}$

$g_{25}$

espaço de escala associado a família de aberturas

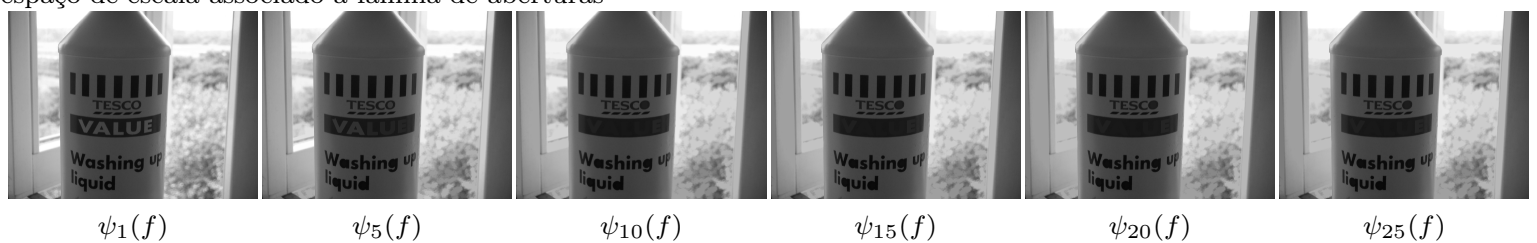

família de marcadores produziada por fechamentos

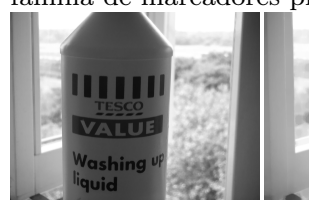

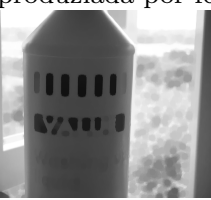

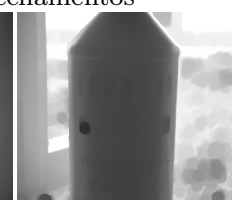

$g_{10}$

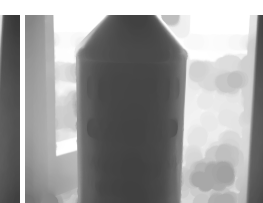

$g_{15}$

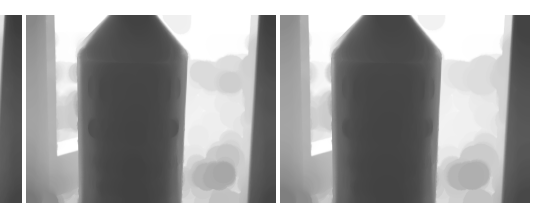

$g_{20}$

$g_{25}$

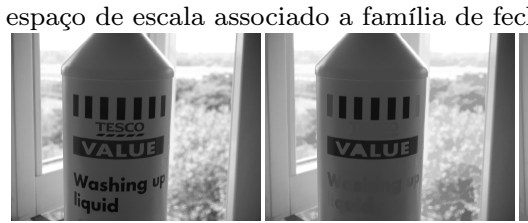

$\psi_{1}(f)$

$\psi_{5}(f)$

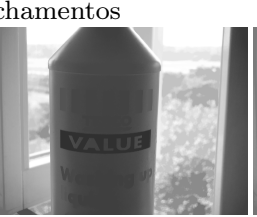

$\psi_{10}(f)$

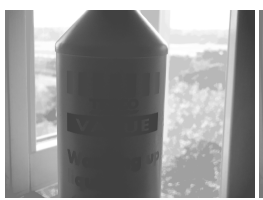

$\psi_{15}(f)$

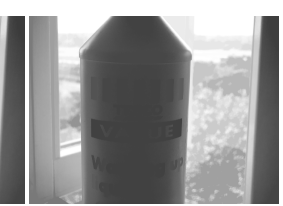

$\psi_{20}(f)$

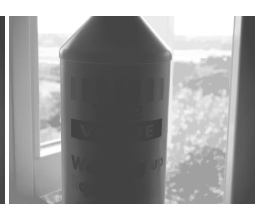

$\psi_{25}(f)$

família de marcadores produziada por filtros de médias

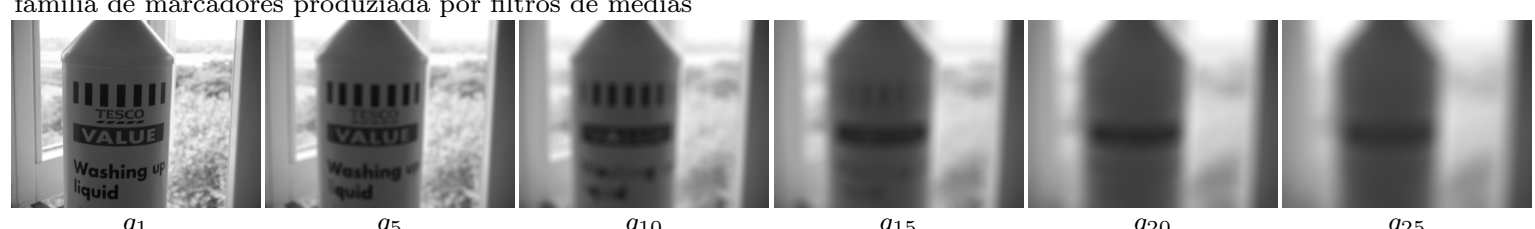

$g_{1}$

$g_{5}$

$g_{10}$

$g_{15}$

$g_{20}$

$g_{25}$

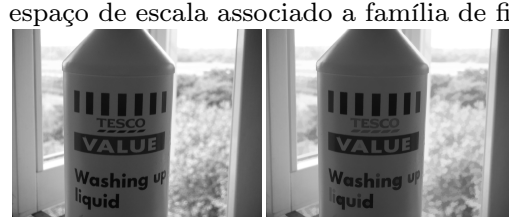

$\psi_{1}(f)$

$\psi_{5}(f)$

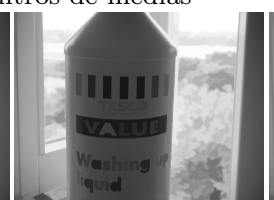

$\psi_{10}(f)$

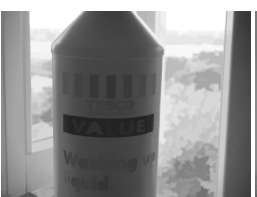

$\psi_{15}(f)$

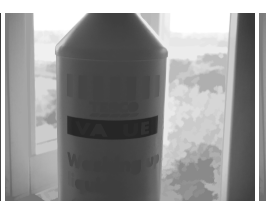

$\psi_{20}(f)$

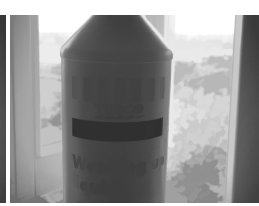

$\psi_{25}(f)$

família de marcadores produziada por filtros sequenciais alternados

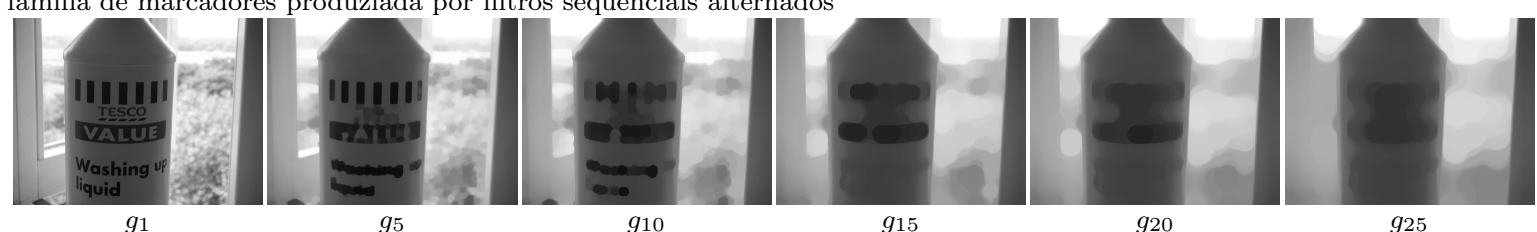

$g_{1}$

$g_{5}$

$g_{10}$

$g_{15}$

$g_{20}$

$g_{25}$

espaço de escala associado a família de filtros sequenciais alternados

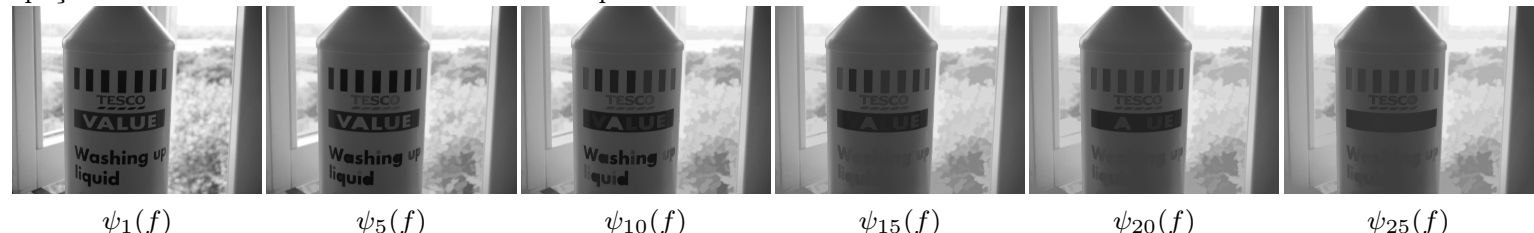

Figura 5.10: Exemplos de imagens de espaços de escalas de levelings associados com famílias de marcadores. 
68 REPRESENTAÇÕES DE ESPAÇO DE ESCALA BASEADO EM LEVELINGS ATRAVÉS DE HIERARQUIAS DOS CONJUNTOS DE NÍVEIS 


\section{Resumo do capítulo}

Neste capítulo é apresentada a classe de operadores residuais últimos levelings, a qual consiste de poderosos operadores residuais estendidos definidos a partir de um espaço de escala baseado em levelings. Um operador desta classe analisa a evolução dos valores residuais presentes em dois operadores consecutivos de um espaço de escala e os maiores valores residuais são considerados. Os valores residuais revelam uma importante informação sobre o contraste de uma imagem. Além dos valores residuais, outras informações associadas com eles podem ser obtidas no momento da extração residual, tais como propriedades das regiões que produziram os valores residuais. Com base nestas considerações, apresenta-se neste capítulo a classe de operadores residuais últimos levelings, na qual é destacado suas propriedades, relações com operadores conhecidos na literatura, definições de novos operadores residuais e algoritmos eficientes para implementação destes operadores.

\subsection{Das últimas aberturas e últimos fechamentos para os últimos levelings}

Como mencionado no Capítulo 1, um operador residual $r$ é definido como a diferença (pixel a pixel) entre dois operadores, digamos $\psi$ e $\phi$, aplicados em uma dada imagem $f \in \mathcal{F}(\mathcal{D})$, ou seja, $r=\psi(f)-\phi(f)$. Assim, $\psi$ e $\phi$ são chamados de primitivas e para um pixel $p \in \mathcal{D},[\psi(f)](p)>$ $[\phi(f)](p)$ implica em um resíduo positivo $r(p)>0 ;[\psi(f)](p)<[\phi(f)](p)$ implica em um resíduo negativo $r(p)<0 ;[\psi(f)](p)=[\phi(f)](p)$ implica em um resíduo nulo $r(p)=0$. Esses operadores tem sido estendidos para extraírem valores residuais de duas famílias de operadores $\left\{\psi_{i}: i \in \mathcal{I}_{\preccurlyeq}\right\}$ e $\left\{\phi_{i}: i \in \mathcal{I}_{\preccurlyeq}\right\}$ indexadas pelo conjunto de índices $\mathcal{I}_{\preccurlyeq}=\left\{0,1, \ldots, \mathcal{I}_{M A X}\right\}$ tal que para quaisquer $i, j \in \mathcal{I}_{\preccurlyeq}$, se $i \leq j$ então $\psi_{i} \preccurlyeq \psi_{j}$ e $\phi_{i} \preccurlyeq \phi_{j}$, onde $\preccurlyeq$ é uma relação de ordem sobre $\mathcal{F}(\mathcal{D})$. Assim, o $i$-ésimo operador residual aplicado em uma imagem $f \in \mathcal{F}(\mathcal{D})$ é definido como $r_{i}(f)=\psi_{i}(f)-\phi_{i}(f)$ e o operador residual estendido $\theta$ aplicado na imagem $f$ é definido como o supremo dos operadores residuais $r_{i}$, isto é

$$
\theta(f)=\sup _{i \in \mathcal{I}_{\preccurlyeq}}\left\{r_{i}(f): \psi_{i}(f)-\phi_{i}(f)\right\} .
$$

Seguindo nesta direção, Beucher (2007) propôs o operador última abertura (por dualidade, o último fechamento), onde as primitivas são aberturas consecutivas, ou seja, $\psi_{i}=\gamma_{i}$ e $\phi_{i}=\gamma_{i+1}$, de 
uma família de aberturas $\left\{\gamma_{i}: i \in I_{\leq}\right\}$indexadas pelo conjunto $\mathcal{I}_{\leq}$. Portanto,

$$
\theta(f)=\sup _{i \in \mathcal{I}_{\leq}}\left\{r_{i}(f): \gamma_{i}(f)-\gamma_{i+1}(f)\right\}
$$

Esta maneira de extrair valores residuais proposta por Beucher (2007) foi usada mais tarde por outros autores para extrair resíduos de famílias de operadores conexos (Alves e Hashimoto , 2014; Alves et al., 2013; Hernandez e Marcotegui, 2011; Marcotegui et al., 2011; Meyer, 2010; Retornaz e Marcotegui, 2007). Assim, objetos contrastados são detectados quando são filtrados por operadores conexos gerando valores residuais conexos. Primeiramente, Retornaz e Marcotegui (2007) propuseram a última abertura por atributo (UAO - do inglês, ultimate attribute opening) $\mathcal{R}_{\theta}^{+}$e por dualidade o último fechamento por atributo (UAC - do inglês, ultimate attribute closing), $\mathcal{R}_{\theta}^{-}$(veja exemplo ilustrado na Figura 6.1) para extrair contraste de objetos presentes em imagens de cenas, ou seja,

$$
\theta(f)=\sup _{i \in \mathcal{I}_{\leq}}\left\{r_{i}(f): r_{i}(f)=\gamma_{i}^{\kappa}(f)-\gamma_{i+1}^{\kappa}(f)\right\}
$$

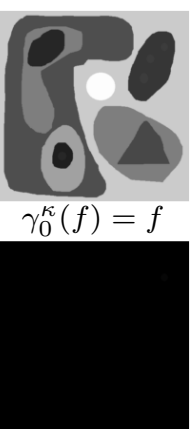

$r_{0}(f)$

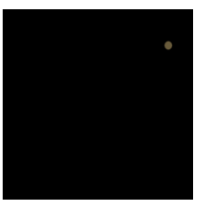

rotulação $r_{0}(f)$

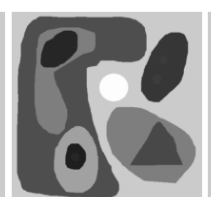

$\gamma_{1}^{\kappa}(f)$

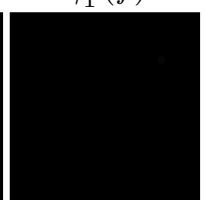

$r_{1}(f)$

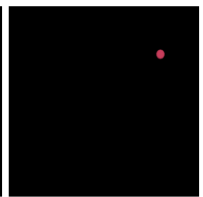

rotulação $r_{1}(f)$

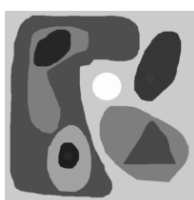

$\gamma_{2}^{\kappa}(f)$

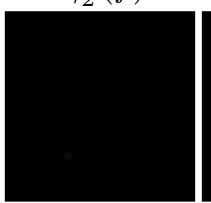

$r_{2}(f)$

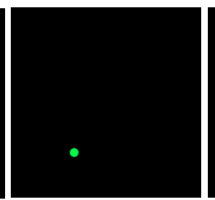

rotulação

$r_{2}(f)$

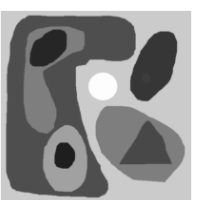

$\gamma_{3}^{\kappa}(f)$

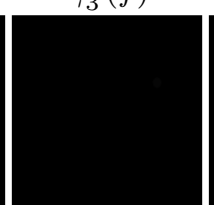

$r_{3}(f)$

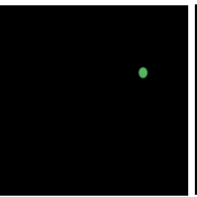

rotulação

$r_{3}(f)$

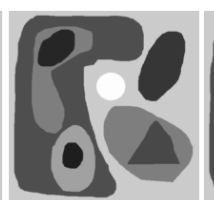

$\gamma_{4}^{\kappa}(f)$

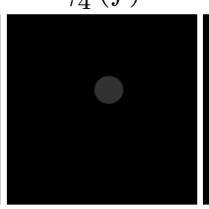

$r_{4}(f)$

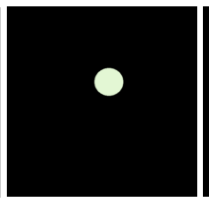

rotulação

$r_{4}(f)$

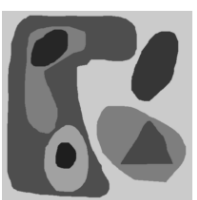

$\gamma_{5}^{\kappa}(f)$

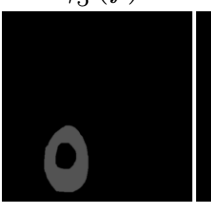

$r_{5}(f)$

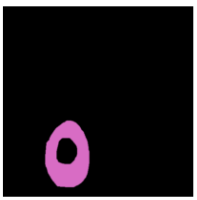

rotulação

$r_{5}(f)$

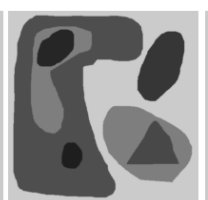

$\gamma_{6}^{\kappa}(f)$

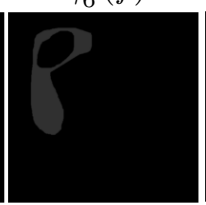

$r_{6}(f)$

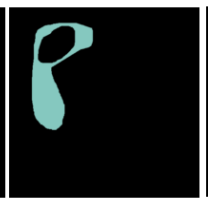

rotulação $r_{6}(f)$

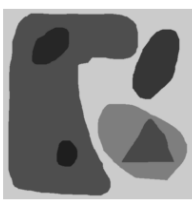

$\gamma_{7}^{\kappa}(f)$

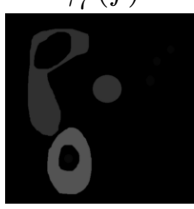

$\sup \left\{r_{i}(f)\right\}$

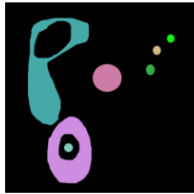

rotulação $\sup \left\{r_{i}(f)\right\}$

Figura 6.1: Um exemplo simples de computação da última abertura por atributo, onde são mostrados: (no topo) a família de primitivas $\left\{\gamma_{0}^{\kappa}(f), \gamma_{1}^{\kappa}(f), \ldots, \gamma_{7}^{\kappa}(f)\right\}$; (no centro) os operadores residuais $\left\{r_{0}(f), r_{1}(f), \ldots, r_{7-1}(f)\right\}$ e o resultado da última abertura por atributo $\sup \left\{r_{i}(f)\right\}$; (em baixo) os operadores residuais rotulados para facilitar a visualização dos valores residuais.

Sabendo que a abertura por atributo pode ser obtida eficientemente usando uma max-tree, Fabrizio e Marcotegui (2009) propuseram um algoritmo eficiente para computar a UAO usando sua estrutura. Depois, Hernandez e Marcotegui (2011) adicionaram um fator de similaridade (função $\Omega: \mathcal{P}(\mathcal{P}(\mathcal{D})) \rightarrow[0,1])$ as zonas planas dos operadores residuais $r_{i}(f)$ para enfatizar as regiões de contraste com base em uma informação de forma. Assim, o $i$-ésimo operador residual com informação de forma é dado por $r_{i}^{\Omega}(f)=r_{i}(f) \times \Omega\left(\mathcal{Z P}\left(r_{i}(f)\right)\right)$ e a última abertura com informação de forma (SUAO - do inglês, shape ultimate attribute opening) é definida por

$$
\mathcal{R}_{\theta}^{+\Omega}(f)=\sup _{i \in \mathcal{I}_{\leq}}\left\{r_{i}^{\Omega}(f): r_{i}^{\Omega}(f)=r_{i}(f) \times \Omega\left(\mathcal{Z P}\left(r_{i}(f)\right)\right) \text { e } r_{i}(f)=\gamma_{i}^{\kappa}(f)-\gamma_{i+1}^{\kappa}(f)\right\}
$$

Diferentemente dessas pesquisas (Fabrizio e Marcotegui, 2009; Hernandez e Marcotegui, 2011; 
Retornaz e Marcotegui, 2007), em Meyer (2010) e depois Alves e Hashimoto (2014), propuseram os últimos levelings por marcadores (ULM - do inglês, ultimate levelings by marked) e os últimos filtros por grãos (UFG - do inglês, ultimate grain filters) para extrair valores residuais de famílias de levelings $\left\{\psi_{i}: i \in \mathcal{I}\right\}$ indexadas pelo conjunto de índices $\mathcal{I}$. Os valores residuais extraídos dessas famílias de primitivas podem produzir valores residuais positivos e negativos em $r_{i}(f)=$ $\psi_{i}(f)-\psi_{i+1}(f)$. Neste caso, são tratados separadamente os valores residuais positivos e negativos, isto é, $r_{i}^{+}(f)=\left[\psi_{i}(f)-\psi_{i+1}(f) \vee 0\right]$ e $r_{i}^{-}(f)=\left[\psi_{i+1}(f)-\psi_{i}(f) \vee 0\right]$. Dessa forma, o operador residual é definido por

$$
\mathcal{R}_{\theta}(f)=\mathcal{R}_{\theta}^{+}(f) \vee \mathcal{R}_{\theta}^{-}(f),
$$

onde $\mathcal{R}_{\theta}^{+}(f)=\sup _{i \in \mathcal{I}}\left\{r_{i}^{+}(f): i \in \mathcal{I}\right\}$ e $\mathcal{R}_{\theta}^{-}(f)=\sup _{i \in \mathcal{I}}\left\{r_{i}^{-}(f): i \in \mathcal{I}\right\}$.

As pesquisas de Meyer (2010) e Alves e Hashimoto (2014) são diferentes da abordagem de Beucher (2007) a qual somente lida com operadores residuais positivos ou negativos. Embora tenham algumas diferenças, estes operadores residuais expostos tem uma importante característica em comum, ou seja, as famílias de primitivas usadas para obtê-los são compostas por levelings consecutivos de espaços de escalas, como apresentado no Capítulo 5. Estes operadores pertencem a uma classe denominada de últimos levelings e definida por primitivas consecutivas de um espaço de escala com base em levelings.

Definição 6.1 (Últimos levelings). Um operador último leveling positivo $\mathcal{R}_{\theta}^{+}$(respectivamente, negativo $\mathcal{R}_{\theta}^{-}$) é o supremo dos resíduos positivos $r_{i}^{+}$(respectivamente, negativos $r_{i}^{-}$) extraído de uma família $\left\{\psi_{i}: i \in \mathcal{I}\right\}$ indexada pelo conjunto de índices $\mathcal{I}=\left\{0,1, \ldots, \mathcal{I}_{M A X}\right\}$, tal que para quaisquer $i, j \in \mathcal{I}, i \leq j \Leftrightarrow \psi_{j}$ é um leveling de $\psi_{i}$. Assim, o operador último leveling $\mathcal{R}_{\theta}$ é o supremo entre $\mathcal{R}_{\theta}^{+}$e $\mathcal{R}_{\theta}^{-}$, isto é

$$
\begin{aligned}
\mathcal{R}_{\theta}^{+}(f)=\sup _{i \in \mathcal{I}}\left\{r_{i}^{+}(f): r_{i}^{+}(f)=\left[\psi_{i}(f)-\psi_{i+1}(f) \vee 0\right]\right\}, \\
\mathcal{R}_{\theta}^{-}(f)=\sup _{i \in \mathcal{I}}\left\{r_{i}^{-}(f): r_{i}^{-}(f)=\left[\psi_{i+1}(f)-\psi_{i}(f) \vee 0\right]\right\}, \\
\mathcal{R}_{\theta}(f)=\mathcal{R}_{\theta}^{+}(f) \vee \mathcal{R}_{\theta}^{-}(f) .
\end{aligned}
$$

Na Figura 6.2 são apresentados alguns exemplos de aplicações de operadores últimos levelings para uma comparação e na Figura 6.3 é apresentado uma ilustração dos operadores da classe dos últimos levelings.

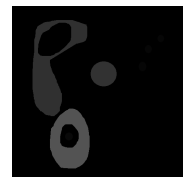

UAO

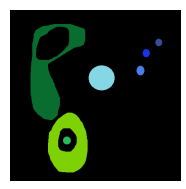

UAO (rotulação)

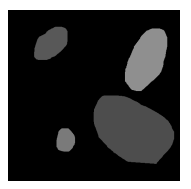

UAC

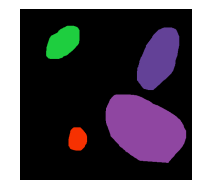

UAC (rotulação)

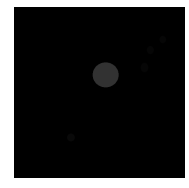

SUAO

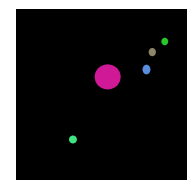

SUAO (rotulação)

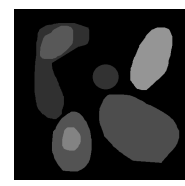

UGF

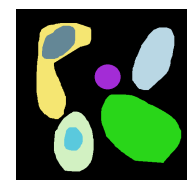

UGF (rotulação)

Figura 6.2: Exemplos de aplicações de últimos levelings: (da esquerda para direita) UAO (Retornaz e Marcotegui, 2007), rotulação da UAO, UAC (Retornaz e Marcotegui, 2007), rotulação da UAC, SUAO com uma função de forma circular (Hernandez e Marcotegui, 2011), rotulação de SUAO, último filtro por grão (UGF - do inglês, ultimate grain filter) (Alves e Hashimoto, 2014), rotulação do $U G F$. 


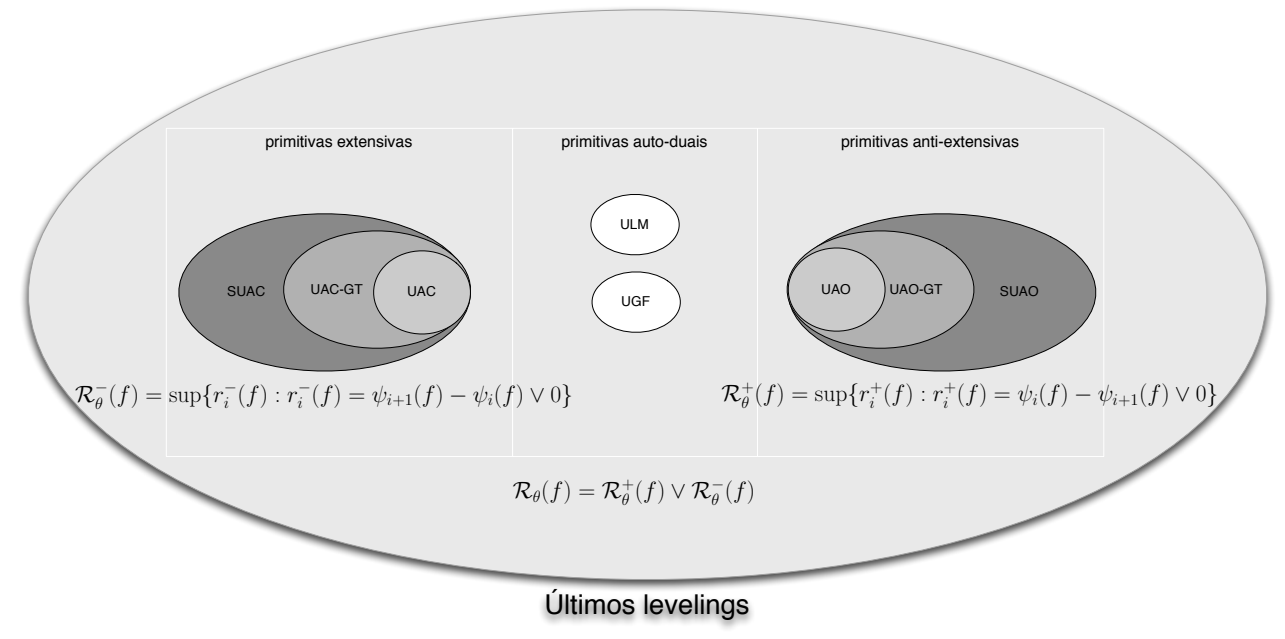

Figura 6.3: Ilustração da classe dos operadores últimos levelings.

\subsection{Propriedade associadas aos operadores últimos levelings}

Os últimos levelings não são filtros morfológicos, ou seja, não são crescentes e também não são idempotente, apesar do $i$-ésimo resíduo $r_{i}^{+}$(respectivamente, $r_{i}^{-}$) ser idempotente, isto é,

$$
\begin{aligned}
r_{i}^{+}\left(r_{i}^{+}(f)\right) & =\psi_{i}\left(r_{i}^{+}(f)\right)-\psi_{i+1}\left(r_{i}^{+}(f)\right) \vee 0 \\
& =\psi_{i}\left(\left[\psi_{i}(f)-\psi_{i+1}(f) \vee 0\right]\right)-\psi_{i+1}\left(\left[\psi_{i}(f)-\psi_{i+1}(f) \vee 0\right]\right) \vee 0 \\
& =\psi_{i}\left(\left[\psi_{i}(f)-\psi_{i+1}(f) \vee 0\right]\right)-0 \\
& =\psi_{i}(f)-\psi_{i+1}(f) \vee 0 \\
& =r_{i}^{+}(f)
\end{aligned}
$$

Apesar disso, os últimos levelings têm propriedades interessantes sobre preservação de estruturas (contornos e extremas regionais) e complementação. Além do mais, os últimos levelings positivos $\mathcal{R}_{\theta}^{+}$ são anti-extensivos, ou seja, $\mathcal{R}_{\theta}^{+}(f) \leq f$. Ademais, a decomposição residual $\left(r_{0}(f), r_{1}(f), \ldots, r_{I_{M A X}}(f)\right)$ de uma imagem $f \in \mathcal{F}(\mathcal{D})$ pode ser usado para construir hierarquias residuais e também permite reconstruir a imagem $f$, pois $r_{i}^{+}(f)=\psi_{i}(f)-\psi_{i+1}(f) \vee 0$ e $r_{i}^{-}(f)=\psi_{i+1}(f)-\psi_{i}(f) \vee 0$ implica em $\psi_{i}(f)=\psi_{i+1}(f)+r_{i}^{+}(f)-r_{i}^{-}(f)$. Assim, pode-se reconstruir a imagem $f$ a partir da decomposição residual da seguinte maneira:

$$
f=\psi_{\mathcal{I}_{M A X}}(f)+\sum_{i \in \mathcal{I}} r_{i}^{+}(f)-\sum_{i \in \mathcal{I}} r_{i}^{-}(f)
$$

A seguir são apresentadas propriedades dos últimos levelings sobre preservação de estruturas e complementação.

\subsubsection{Propriedades sobre preservação de estruturas}

Se a primitiva $\psi_{i+1}(f)$ é um leveling de uma primitiva $\psi_{i}(f)$ e $(p, q) \in \mathcal{A}$, então $\left[\psi_{i}(f)\right](p)=$ $\left[\psi_{i}(f)\right](q) \Rightarrow\left[\psi_{i+1}(f)\right](p)=\left[\psi_{i+1}(f)\right](q)$ o que implica em $\left[0 \vee \psi_{i}(f)-\psi_{i+1}(f)\right](p)=\left[0 \vee \psi_{i}(f)-\right.$ $\left.\psi_{i+1}(f)\right](q)$ (respectivamente, $\left.\left[0 \vee \psi_{i+1}(f)-\psi_{i}(f)\right](p)=\left[0 \vee \psi_{i+1}(f)-\psi_{i}(f)\right](q)\right)$ mostrando que $r_{i}^{+}(f)$ (respectivamente, $\left.r_{i}^{-}(f)\right)$ é conexo de $\psi_{i}(f)$ o que leva ao Lema 6.2. 
Lema 6.2. Se $\left(\psi_{0}(f), \psi_{1}(f), \ldots, \psi_{\mathcal{I}_{M A X}}(f)\right)$ é um espaço de escala baseado em levelings, então $r_{i}^{+}(f)=\left[0 \vee \psi_{i}(f)-\psi_{i+1}(f)\right]$ (respectivamente, $\left.r_{i}^{-}(f)\right)$ é um operador conexo para todo $i \in \mathcal{I}$.

Prova: Seja $(p, q) \in \mathcal{A}$ dois pixels adjacentes. Como os operadores levelings são conexos (pois satisfazem a Definição 3.2 de conexo), então segue que $\left[\psi_{i}(f)\right](p)=\left[\psi_{i}(f)\right](q) \Rightarrow\left[\psi_{i+1}(f)\right](p)=$ $\left[\psi_{i+1}(f)\right](q)$ implicando ainda em $\left[0 \vee \psi_{i}(f)-\psi_{i+1}(f)\right](p)=\left[0 \vee \psi_{i}(f)-\psi_{i+1}(f)\right](q)$. Logo, $\left[\psi_{i}(f)\right](p)=$ $\left[\psi_{i}(f)\right](q) \Rightarrow\left[r_{i}^{+}(f)\right](p)=\left[r_{i}^{+}(f)\right](p)$, mostrando que $r_{i}$ é um operador conexo de $\psi_{i}(f)$ e por transitividade de $f$, pois $f(p)=f(q) \Rightarrow\left[\psi_{i}(f)\right](p)=\left[\psi_{i}(f)\right](q) \Rightarrow\left[r_{i}^{+}(f)\right](p)=\left[r_{i}^{+}(f)\right](p)$.

Além disso, se $\left[\psi_{i+1}(f)\right](p)>\left[\psi_{i+1}(f)\right](q)$, então $\left[\psi_{i}(f)\right](p) \geq\left[\psi_{i+1}(f)\right](p)>\left[\psi_{i+1}(f)\right](q) \geq$ $\left[\psi_{i}(f)\right](q)$, pois $\psi_{i+1}(f)$ é um leveling de $\psi_{i}(f)$. Logo, pode-se derivar duas afirmações:

(i) $\quad\left[0 \vee \psi_{i}(f)-\psi_{i+1}(f)\right](p) \geq\left[0 \vee \psi_{i}(f)-\psi_{i+1}(f)\right](q) \quad$ (ou seja, $\left.\left[r_{i}^{+}(f)\right](p) \geq\left[r_{i}^{+}(f)\right](q)\right)$;

(ii) $\left[\psi_{i}(f)\right](p)>\left[\psi_{i}(f)\right](q)$.

Portanto, isto mostra que quando existe uma transição em $\psi_{i+1}(f)$, então pode-se gerar uma transição no resíduo positivo $r_{i}^{+}(f)$, implicando também em uma transição em $\psi_{i}(f)$ no mesmo lugar e com a mesma ordem. Pode-se chegar a mesma conclusão, se considerarmos $\left[\psi_{i+1}(f)\right](p)=\left[\psi_{i+1}(f)\right](q)$. Portanto,

$$
\forall(p, q) \in \mathcal{A},\left[r_{i}^{+}(f)\right](p)>\left[r_{i}^{+}(f)\right](q) \Rightarrow\left[\psi_{i}(f)\right](p)>\left[\psi_{i}(f)\right](q)
$$

o que mostra também que $r_{i}^{+}(f)$ (respectivamente, $r_{i}^{-}(f)$ ) é um operador monotone planing de $\psi_{i}(f)$. Isto leva ao Lema 6.3.

Lema 6.3. Se $\left(\psi_{0}(f), \psi_{1}(f), \ldots, \psi_{\mathcal{I}_{M A X}}(f)\right)$ é um espaço de escala baseado em levelings, então $r_{i}^{+}(f)=\left[0 \vee \psi_{i}(f)-\psi_{i+1}(f)\right]$ (respectivamente, $r_{i}^{-}(f)$ ) é um operador monotone planing para todo $i \in \mathcal{I}$.

Prova: Seja $(p, q) \in \mathcal{A}$ dois pixels vizinhos. Vamos analisar os casos quando: $\left[\psi_{i+1}(f)\right](p)=$ $\left[\psi_{i+1}(f)\right](q)$ e $\left[\psi_{i+1}(f)\right](p) \neq\left[\psi_{i+1}(f)\right](q)$.

- Se $\left[\psi_{i+1}(f)\right](p) \neq\left[\psi_{i+1}(f)\right](q)$.

Então, sem perda de generalidade, suponha $\left[\psi_{i+1}(f)\right](p)>\left[\psi_{i+1}(f)\right](q)$. Logo, como $\psi_{i+1}(f)$ é um leveling de $\psi_{i}(f)$, segue que $\left[\psi_{i}(f)\right](p) \geq\left[\psi_{i+1}(f)\right](p)>\left[\psi_{i+1}(f)\right](q) \geq\left[\psi_{i}(f)\right](q)$. Consequentemente, temos:

- $\left[\psi_{i}(f)\right](p)-\left[\psi_{i+1}(f)\right](p) \geq 0$, implicando em $\left[r_{i}^{+}(f)\right](p) \geq 0$.

$-\left[\psi_{i}(f)\right](q)-\left[\psi_{i+1}(f)\right](q) \leq 0$, implicando em $\left[r_{i}^{+}(f)\right](q)=0$.

Portanto, se $\left[r_{i}^{+}(f)\right](p)>\left[r_{i}^{+}(f)\right](q)$ então $\left[\psi_{i}(f)\right](p)>\left[\psi_{i}(f)\right](q)$, o que mostra que $r_{i}^{+}(f)$ é um monotone planing de $\psi_{i}(f)$.

- Se $\left[\psi_{i+1}(f)\right](p)=\left[\psi_{i+1}(f)\right](q)$.

Então, teremos dois casos para analisar: ou $\left[\psi_{i}(f)\right](p) \neq\left[\psi_{i}(f)\right](q)$, ou $\left[\psi_{i}(f)\right](p)=\left[\psi_{i}(f)\right](q)$.

- Se $\left[\psi_{i}(f)\right](p)=\left[\psi_{i}(f)\right](q)$ então $\left[\psi_{i}(f)\right](p)-\left[\psi_{i+1}(f)\right](p)=\left[\psi_{i}(f)\right](q)-\left[\psi_{i+1}(f)\right](q)$, pois $\left[\psi_{i+1}(f)\right](p)=\left[\psi_{i+1}(f)\right](q)$ e $\left[\psi_{i}(f)\right](p)=\left[\psi_{i}(f)\right](q)$. Logo, $\left[r_{i}^{+}(f)\right](p)=\left[r_{i}^{+}(f)\right](q)$ e $\left[\psi_{i}(f)\right](p)=\left[\psi_{i}(f)\right](q)$, o que mostra que $r_{i}^{+}(f)$ é um monotone planing de $\psi_{i}(f)$ por vacuidade. 
- Se $\left[\psi_{i}(f)\right](p) \neq\left[\psi_{i}(f)\right](q)$. Então, suponha sem perda de generalidade que $\left[\psi_{i}(f)\right](p)>$ $\left[\psi_{i}(f)\right](q)$. Logo, ou $\left[\psi_{i}(f)\right](p)>\left[\psi_{i+1}(f)\right](p)$, ou $\left[\psi_{i}(f)\right](p)=\left[\psi_{i+1}(f)\right](p)$, ou $\left[\psi_{i}(f)\right](p)<$ $\left[\psi_{i+1}(f)\right](p)$.

* Se $\left[\psi_{i}(f)\right](p)=\left[\psi_{i+1}(f)\right](p)$ então $\left[\psi_{i}(f)\right](p)-\left[\psi_{i+1}(f)\right](p)=0$ e $\left[\psi_{i}(f)\right](q)-$ $\left[\psi_{i+1}(f)\right](q)<0$, pois $\left[\psi_{i}(f)\right](q)<\left[\psi_{i}(f)\right](p)=\left[\psi_{i+1}(f)\right](p)=\left[\psi_{i+1}(f)\right](q)$. Logo, $\left[r_{i}^{+}(f)\right](p)=\left[r_{i}^{+}(f)\right](q)$ e $\left[\psi_{i}(f)\right](p)>\left[\psi_{i}(f)\right](q)$ o que mostra que $r_{i}^{+}(f)$ é um monotone planing de $\psi_{i}(f)$ por vacuidade.

* Se $\left[\psi_{i}(f)\right](p)>\left[\psi_{i+1}(f)\right](p)$ então $\left[\psi_{i}(f)\right](p)-\left[\psi_{i+1}(f)\right](p)>0$ e $\left[\psi_{i}(f)\right](q)-$ $\left[\psi_{i+1}(f)\right](q)$, pode ser maior, menor ou igual a zero. Logo, mesmo assim, temos que $\left[\psi_{i}(f)\right](p)-\left[\psi_{i+1}(f)\right](p)>\left[\psi_{i}(f)\right](q)-\left[\psi_{i+1}(f)\right](q)$, pois $\left[\psi_{i}(f)\right](p)>\left[\psi_{i}(f)\right](q)$ e $\left[\psi_{i+1}(f)\right](p)=\left[\psi_{i+1}(f)\right](q)$, o que mostra que $r_{i}^{+}(f)$ é um monotone planing de $\psi_{i}(f)$.

* Se $\left[\psi_{i}(f)\right](p)<\left[\psi_{i+1}(f)\right](p)$ então $\left[\psi_{i}(f)\right](p)-\left[\psi_{i+1}(f)\right](p)<0$ e $\left[\psi_{i}(f)\right](q)-$ $\left[\psi_{i+1}(f)\right](q)<0$, pois $\left[\psi_{i+1}(f)\right](q)=\left[\psi_{i+1}(f)\right](p)>\left[\psi_{i}(f)\right](p)>\left[\psi_{i}(f)\right](q)$. Logo, $\left[r_{i}^{+}(f)\right](p)=\left[r_{i}^{+}(f)\right](q)$ e $\left[\psi_{i}(f)\right](p)>\left[\psi_{i}(f)\right](q)$, o que mostra que $r_{i}^{+}(f)$ é um monotone planing de $\psi_{i}(f)$.

E assim concluímos que $\forall(p, q) \in \mathcal{A}$ vizinhos, se $r_{i}^{+}(p)>r_{i}^{+}(q)$ então $\psi_{i}(p)>\psi_{i}(q)$ o que mostra que $r_{i}^{+}$é um monotone planing de $\psi_{i}$ e por transitividade $r_{i}^{+}$também é um monotone planing de $f$, pois $r_{i}^{+}(p)>r_{i}^{+}(q) \Rightarrow \psi_{i}(p)>\psi_{i}(q) \Rightarrow f(p)>f(q)$.

Como mostrado nas Proposições 3.12, 3.14 e 3.13, os operadores conexos, monotone planings e levelings são fechados sobre a operação de supremo. Logo, o supremo dos $i$-ésimos resíduos positivos $r_{i}^{+}$e negativos $r_{i}^{-}$são operadores conexos. Mais ainda, o supremo dos $i$-ésimos resíduos positivos $r_{i}^{+}$(respectivamente, negativos $r_{i}^{-}$) são operadores monotone planings. Isso leva às Proposições 6.4 e 6.5 .

Proposição 6.4. Os últimos levelings são operadores conexos.

Prova: Seguindo a Definição 6.1, um último leveling de uma imagem $f \in \mathcal{F}(\mathcal{D})$ é dado por $\mathcal{R}_{\theta}(f)=\mathcal{R}_{\theta}^{+}(f) \vee \mathcal{R}_{\theta}^{-}(f)$, onde $\mathcal{R}_{\theta}^{+}(f)$ e $\mathcal{R}_{\theta}^{-}(f)$ são os supremos dos $i$-ésimos resíduos $r_{i}^{+}(f)$ e $r_{i}^{+}(f)$. Então, pela Proposição 6.2 os $i$-ésimos resíduos $r_{i}^{+}(f)$ e $r_{i}^{+}(f)$ são operadores conexos e pela Proposição 3.12 o supremo de operadores conexos é um operador conexo. Portanto, $\mathcal{R}_{\theta}^{+}(f)$ e $\mathcal{R}_{\theta}^{+}(f)$ são operadores conexos e consequentemente $\mathcal{R}_{\theta}(f)$ é um operador conexo.

Proposição 6.5. Os últimos levelings positivos (respectivamente, negativos) são operadores monotone planing.

Prova: Seguindo a Definição 6.1, um último leveling positivo $\mathcal{R}_{\theta}^{+}(f)$ é dado pelo supremo dos $i$-ésimos resíduos $r_{i}^{+}(f)$. Então, graças a Proposição 6.3, os operadores $r_{i}^{+}$são operadores monotone planings e pela Proposição 3.14 o supremo de operadores monotone planings é um operador monotone planing. Portanto, os últimos levelings positivos são monotone planings.

Consequentemente, as Proposições 6.4 e 6.5 levam aos Corolários 6.6 e 6.7.

Corolário 6.6. Os últimos filtros por grãos são operadores conexos.

Corolário 6.7. As últimas aberturas (respectivamente, fechamentos) por atributos são operadores monotone planings. 
Como os últimos levelings positivos (respectivamente, negativos) são operadores monotone planings e portanto herdam a propriedade de não criarem máximos regionais (respectivamente, mínimos), e isso leva à Proposição 6.8 e consequentemente ao Corolário 6.9.

Proposição 6.8. Se $\mathcal{R}_{\theta}^{+}(f)$ (respectivamente, $\mathcal{R}_{\theta}^{-}(f)$ ) é um último leveling positivo (respectivamente, negativo) contendo uma máxima regional $\mathcal{X} \subseteq \mathcal{D}$, então $f$ possui uma máxima (respectivamente, minima) regional $\mathcal{Z} \subseteq \mathcal{X}$.

Prova: Esta Proposição é análoga a Proposição 3.8, sendo que a única diferença é que as máximas regionais $\mathcal{X}$ dos últimos levelings negativos implicam em uma mínima regional $\mathcal{Z} \subseteq \mathcal{X}$ da imagem $f$. Tendo em mente esta consideração, a prova é a mesma.

Corolário 6.9. Se $\mathcal{R}_{\theta}^{+}(f)$ (respectivamente, $\mathcal{R}_{\theta}^{-}(f)$ ) é uma abertura (respectivamente, fechamento) por atributo de uma imagem $f \in \mathcal{F}(\mathcal{D})$ contendo uma máxima (respectivamente, minima) regional $\mathcal{X} \subseteq \mathcal{D}$, então $f$ possui uma máxima (respectivamente, minima) regional $\mathcal{Z} \subseteq \mathcal{X}$.

\subsubsection{Propriedade a respeito de complementação}

Relembrando, dois operadores $\psi$ e $\varphi$ são duais com respeito à complementação se ao aplicar $\psi$ em uma imagem $f \in \mathcal{F}(\mathcal{D})$ equivale em aplicar $\varphi$ no complemento de $f$ e tomar o complemento de $\varphi$, isto é, $\psi(f)=\left[\varphi\left(f^{c}\right)\right]^{c}$. Similarmente, $\psi$ e $\varphi$ são complementares se ao aplicar $\psi$ na imagem $f$ equivale a aplicar $\varphi$ no complemento de $f$, isto é, $\psi(f)=\varphi\left(f^{c}\right)$. Neste sentido, os últimos levelings respeitam a complementariedade das primitivas e isto leva à Proposição 6.10 e consequentemente ao Corolário 6.11.

Proposição 6.10. Se $\left(\psi_{0}(f), \psi_{1}(f), \ldots, \psi_{\mathcal{I}_{M A X}}(f)\right)$ e $\left(\varphi_{0}(f), \varphi_{1}(f), \ldots, \varphi_{\mathcal{I}_{M A X}}(f)\right)$ são dois espaços de escalas baseados em levelings satisfazendo $\psi_{i}(f)$ é dual de $\varphi_{i}(f)$ para todo $i \in \mathcal{I}$, então $r_{i}^{+}(f)$ e $r_{i}^{-}(f)$ são complementares, onde $r_{i}^{+}(f)=\left[0 \vee \psi_{i}(f)-\psi_{i+1}(f)\right]$ e $r_{i}^{-}(f)=\left[0 \vee \varphi_{i+1}(f)-\varphi_{i}(f)\right]$. Prova:

$$
\begin{aligned}
r_{i}^{+}(f) & =\left[0 \vee \psi_{i}(f)-\psi_{i+1}(f)\right] \\
& =\left[0 \vee\left(\varphi_{i}\left(f^{c}\right)\right)^{c}-\left(\varphi_{i+1}\left(f^{c}\right)\right)^{c}\right] \\
& =\left[0 \vee\left(K-\varphi_{i}\left(f^{c}\right)\right)-\left(K-\varphi_{i+1}\left(f^{c}\right)\right)\right] \\
& =\left[0 \vee \varphi_{i+1}\left(f^{c}\right)-\varphi_{i}\left(f^{c}\right)\right] \\
& =r_{i}^{-}\left(f^{c}\right) .
\end{aligned}
$$

Corolário 6.11. A última abertura por atributo e o último fechamento por atributo são complementares.

Analogamente, um operador $\psi$ é auto-dual com respeito a complementação se o seu operador dual é si mesmo, ou seja, $\psi(f)=\left[\psi\left(f^{c}\right)\right]^{c}$. Similarmente, $\psi$ é auto-complementar se $\psi(f)=\psi\left(f^{c}\right)$. Neste sentido, deriva-se as Proposições 6.12 e consequentemente os Corolários 6.13 e 6.14.

Proposição 6.12. Se $\left(\psi_{0}(f), \psi_{1}(f), \ldots, \psi_{\mathcal{I}_{M A X}}(f)\right)$ é um espaço de escala baseado em levelings tal que $\psi_{i}(f)$ é auto-dual, então $r_{i}(f)=r_{i}^{+}(f) \vee r_{i}^{-}(f)$ é auto-complementar.

Prova:

$$
\begin{array}{rlr}
r_{i}(f) & =r_{i}^{+}(f) \vee r_{i}^{-}(f) \\
& =r_{i}^{-}\left(f^{c}\right) \vee r_{i}^{+}\left(f^{c}\right) & \\
& =r_{i}\left(f^{c}\right)
\end{array}
$$


Corolário 6.13. Os últimos filtros por grãos são auto-complementares.

Corolário 6.14. Se $\mathcal{R}_{\theta}^{+}(f)$ é uma última abertura por atributo de uma imagem $f \in \mathcal{F}(\mathcal{D})$ e $\mathcal{R}_{\theta}^{-}(f)$ é um último fechamento por atributo de $f$, então $\left[\mathcal{R}_{\theta}^{+}(f) \vee \mathcal{R}_{\theta}^{-}(f)\right]$ é auto-complementar.

\subsection{Algoritmo para construção dos últimos levelings}

Graças ao Teorema 5.12 sabemos que uma sequência $\left(\psi_{0}(f), \psi_{1}(f), \ldots, \psi_{I_{M A X}(f)}\right)$ de reconstruções de uma sequência de árvores $\left(\mathcal{T}_{f}^{0}, \mathcal{T}_{f}^{1}, \ldots, \mathcal{T}_{f}^{\mathcal{I}_{M A X}}\right.$ ) obtidas por podas sucessivas (ver Definição 5.11) de uma árvore $\mathcal{T}_{f}$ é um espaço de escala baseado em levelings. A Figura 6.4 ilustra para uma árvore $\mathcal{T}_{f}$, a obtenção dos operadores $\psi_{i}(f)=\operatorname{Rec}\left(\mathcal{T}_{f}^{i}\right)$, e das sucessivas operações de podas $\mathcal{T}_{f}^{0}=\mathcal{T}_{f}$ e $\mathcal{T}_{f}^{i}=\mathcal{P} \operatorname{oda}\left(\mathcal{T}_{f}^{i-1}\right)$, para todo $i \in \mathcal{I}$. Assim, pode-se usar os vértices de $\mathcal{T}_{f}^{i}$ que não estão

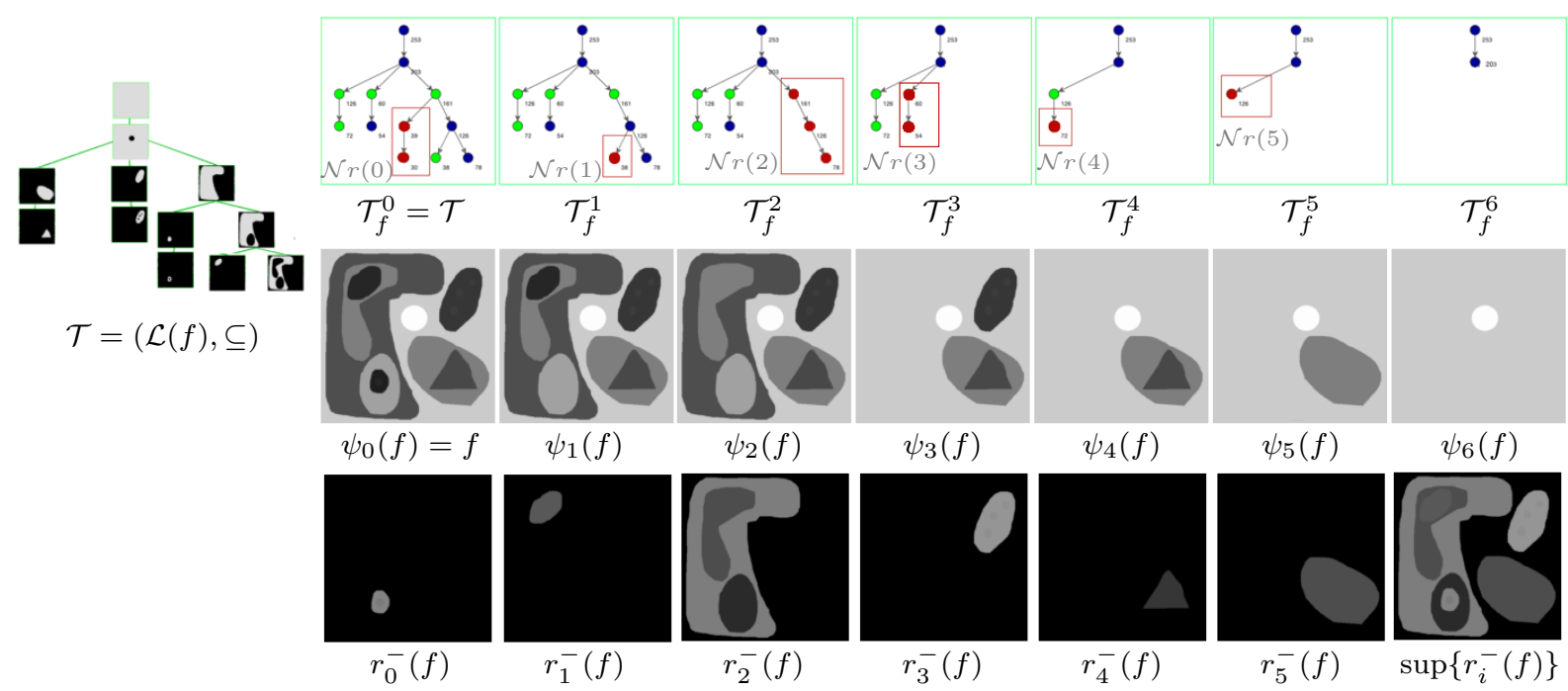

Figura 6.4: Um exemplo de computação do último fechamento por atributo onde são mostrados: (no topo) as árvore derivadas por podas sucessivas; (no centro) as família de primitivas $\left\{\psi_{i}(f): \psi_{i}(f)=\operatorname{Rec}\left(\mathcal{T}_{f}^{i}\right), i=\right.$ $0,1, \ldots, 6\}$; (em baixo) os i-ésimos operadores residuais $\left\{r_{0}^{-}(f), r_{1}^{-}(f), \ldots, r_{6-1}^{-}(f)\right\}$ extraídos da família de primitivas e o resultado do último fechamento por atributo $\sup \left\{r_{i}(f)\right\}$.

em $\mathcal{T}_{f}^{i+1}$ para calcular os valores residuais entre $\psi_{i}(f)$ e $\psi_{i+1}(f)$. Note que, $\forall p \in \mathcal{D}$,

$$
\begin{aligned}
& {\left[r_{i}^{+}(f)\right](p)=\operatorname{level}\left(\mathcal{S C}\left(\mathcal{T}_{f}^{i}, p\right)\right)-\operatorname{level}\left(\mathcal{S C}\left(\mathcal{T}_{f}^{i+1}, p\right)\right) \vee 0} \\
& {\left[r_{i}^{-}(f)\right](p)=\operatorname{level}\left(\mathcal{S C}\left(\mathcal{T}_{f}^{i+1}, p\right)\right)-\operatorname{level}\left(\mathcal{S C}\left(\mathcal{T}_{f}^{i}, p\right)\right) \vee 0}
\end{aligned}
$$

Então, considere $\mathcal{N} r(i)$ o conjunto de vértices removidos da árvore $\mathcal{T}_{f}^{i}$ para construir a versão podada $\mathcal{T}_{f}^{i+1}$, ou seja, $\mathcal{N} r(i)=\mathcal{T}_{f}^{i} \backslash \mathcal{T}_{f}^{i+1}$. Dessa forma, como as árvores são derivadas por podas sucessivas, pode-se estabelecer à Proposição 6.15, que garante que os $i$-ésimos operadores residuais $r_{i}$ podem ser computados paralelamente.

Proposição 6.15. Se $i, j \in \mathcal{I}$ tal que $i \neq j$, então $\mathcal{N} r(i) \cap \mathcal{N} r(j)=\emptyset$.

Prova: Prova por contradição.

Suponha que $\mathcal{N} r(i) \cap \mathcal{N} r(j) \neq \emptyset$. Então, por definição de $\mathcal{N} r(i)$ e $\mathcal{N} r(j)$, segue que:

$$
\mathcal{N} r(i)=\mathcal{T}_{f}^{i} \backslash \mathcal{T}_{f}^{i+1} \text { e } \mathcal{N} r(j)=\mathcal{T}_{f}^{j} \backslash \mathcal{T}_{f}^{j+1}
$$


Como a sequência de árvores $\left(\mathcal{T}_{f}^{0}, \mathcal{T}_{f}^{1}, \ldots, \mathcal{T}_{f}^{\mathcal{I}_{M A X}}\right)$ são obtidas por podas sucessivas de $\mathcal{T}_{f}=\mathcal{T}_{f}^{0}$, então $\mathcal{T}_{f}^{i+1} \subset \mathcal{T}_{f}^{i}$ e $\mathcal{T}_{f}^{j+1} \subset \mathcal{T}_{f}^{j}$. Logo,

$$
\begin{array}{lll}
\mathcal{N} r(i) \subseteq \mathcal{T}_{f}^{i} & \text { e } & \mathcal{N} r(i) \cap \mathcal{T}_{f}^{i+1}=\emptyset \\
\mathcal{N} r(j) \subseteq \mathcal{T}_{f}^{j} & \text { e } & \mathcal{N} r(j) \cap \mathcal{T}_{f}^{j+1}=\emptyset
\end{array}
$$

Portanto, sem perda de generalidade, suponha $i>j$, e como todas as árvores são derivadas por podas sucessivas, segue que $\mathcal{T}_{f}^{i} \subset \mathcal{T}_{f}^{j}$ e consequentemente $\mathcal{N} r(i) \subseteq \mathcal{T}_{f}^{i}$ e $\mathcal{N} r(i) \cap \mathcal{T}_{f}^{j}=\emptyset$, o que é uma contradição, pois $\mathcal{N} r(i) \cap \mathcal{N} r(j) \neq \emptyset$ e $\mathcal{N} r(j) \subseteq \mathcal{T}_{f}^{j}$.

Portanto, pode-se calcular os valores residuais positivos (respectivamente, negativos) de todos os pixels de um dado vértice $C_{1} \in \mathcal{N} r(i)$ subtraindo o seu nível com o nível do primeiro vértice ancestral $C_{k}$ de $C_{1}$ que não está presente em $\mathcal{N} r(i)$, isto é, [level $\left.\left(C_{1}\right)-\operatorname{level}\left(C_{k}\right) \vee 0\right]$. Mas é claro, se existirem vértices $C_{2}, C_{3}, \ldots, C_{k-1}$ entre $C_{1}$ e $C_{k}$ dentro de $\mathcal{N} r(i)$, ou seja, $C_{1} \subset C_{2} \subset \ldots \subset C_{k-1} \subset C_{k}$ então pela Proposição 6.16 vale que: $\left[\operatorname{level}\left(C_{1}\right)-\operatorname{level}\left(C_{k}\right)\right]=\left[\operatorname{level}\left(C_{1}\right)-\operatorname{level}\left(C_{2}\right)\right]+\ldots+\left[\operatorname{level}\left(C_{k-1}\right)-\right.$ level $\left.\left(C_{k}\right)\right]$.

Proposição 6.16. As somas das diferenças consecutivas de uma sequência (level $\left(C_{1}\right)$, level $\left(C_{2}\right)$, $\ldots$, level $\left.\left(C_{k}\right)\right)$ é dada pela diferença do primeiro com o último termo da sequência. Em outras palavras, level $\left(C_{1}\right)-\operatorname{level}\left(C_{k}\right)=\left(\sum_{i=1}^{k-1} \operatorname{level}\left(C_{i}\right)-\operatorname{level}\left(C_{i+1}\right)\right)$.

Prova: Prova por indução em $k$.

A base da indução é para $k=2$ e assim: level $\left(C_{1}\right)-\operatorname{level}\left(C_{2}\right)=\left(\sum_{i=1}^{2-1} \operatorname{level}\left(C_{i}\right)-\operatorname{level}\left(C_{i+1}\right)\right)$.

Suponha por hipótese de indução (HI) que:

$$
k>2 \text { e level }\left(C_{1}\right)-\operatorname{level}\left(C_{k}\right)=\sum_{i=1}^{k-1} \operatorname{level}\left(C_{i}\right)-\operatorname{level}\left(C_{i+1}\right)
$$

Portanto, segue que:

$$
\begin{aligned}
\operatorname{level}\left(C_{1}\right)-\operatorname{level}\left(C_{k+1}\right) & =\left(\sum_{i=1}^{k} \operatorname{level}\left(C_{i}\right)-\operatorname{level}\left(C_{i+1}\right)\right)+\left(\operatorname{level}\left(C_{k}\right)-\operatorname{level}\left(C_{k+1}\right)\right) \\
& =\left(\operatorname{level}\left(C_{1}\right)-\operatorname{level}\left(C_{k}\right)\right)+\left(\operatorname{level}\left(C_{k}\right)-\operatorname{level}\left(C_{k+1}\right)\right) \\
& =\operatorname{level}\left(C_{1}\right)-\operatorname{level}\left(C_{k+1}\right)
\end{aligned}
$$

Assim, pode-se derivar as seguintes recorrências para calcular os valores residuais presentes em cada vértice $C \in \mathcal{N} r(i)$, isto é:

$$
\begin{aligned}
& r_{\mathcal{T}_{f}^{i}}^{+}(C)= \begin{cases}{[\operatorname{level}(C)-\operatorname{level}(\operatorname{Pai}(C)) \vee 0],} & \text { se Pai }(C) \notin \mathcal{N} r(i), \\
{[\operatorname{level}(C)-\operatorname{level}(\operatorname{Pai}(C)) \vee 0]+r_{\mathcal{T}_{f}^{i}}^{+}(\operatorname{Pai}(C)),} & \text { caso contrário, }\end{cases} \\
& r_{\mathcal{T}_{f}^{i}}^{-}(C)= \begin{cases}{[\operatorname{level}(\operatorname{Pai}(C))-\operatorname{level}(C) \vee 0],} & \text { se Pai }(C) \notin \mathcal{N} r(i), \\
{[\operatorname{level}(\operatorname{Pai}(C))-\operatorname{level}(C) \vee 0]+r_{\mathcal{T}_{f}^{i}}^{-}(\operatorname{Pai}(C)),} & \text { caso contrário, }\end{cases}
\end{aligned}
$$

e assim os $i$-ésimos resíduos $r_{i}^{+}(f)$ e $r_{i}^{-}(f)$ podem ser computados pelas Equações 6.10 e 6.11 usando 
os menores componentes (ou formas) de $\mathcal{T}_{f}^{i}$ quando eles estão em $\mathcal{N} r(i)$, isto é:

$$
\begin{aligned}
& \forall p \in \mathcal{D},\left[r_{i}^{+}(f)\right](p)= \begin{cases}r_{\mathcal{T}_{f}^{i}}^{+}\left(\mathcal{S C}\left(\mathcal{T}_{f}^{i}, p\right)\right), & \text { se } \mathcal{S C}\left(\mathcal{T}_{f}^{i}, p\right) \in \mathcal{N} r(i), \\
0, & \text { caso contrário, }\end{cases} \\
& \forall p \in \mathcal{D},\left[r_{i}^{-}(f)\right](p)= \begin{cases}r_{\mathcal{T}_{f}^{i}}^{-}\left(\mathcal{S C}\left(\mathcal{T}_{f}^{i}, p\right)\right), & \text { se } \mathcal{S C}\left(\mathcal{T}_{f}^{i}, p\right) \in \mathcal{N} r(i), \\
0, & \text { caso contrário. }\end{cases}
\end{aligned}
$$

Consequentemente, isto leva ao Algoritmo 6.1 para computar o último leveling por meio de uma abordagem top-down, semelhante ao algoritmo proposto por Fabrizio e Marcotegui (2009) para computar a última abertura por atributo, isto é: $(i)$ o resíduo do vértice pai é calculado e, $(i i)$ propaga-se para os vértices filhos, (iii) para cada vértice filho calcula-se seu resíduo e o compara com o resíduo do vértice pai, e mantem atualizados os máximos resíduos em $\mathcal{R}_{\theta}$. Depois, (iv) cada vértice filho se torna pai e o processo se repete.

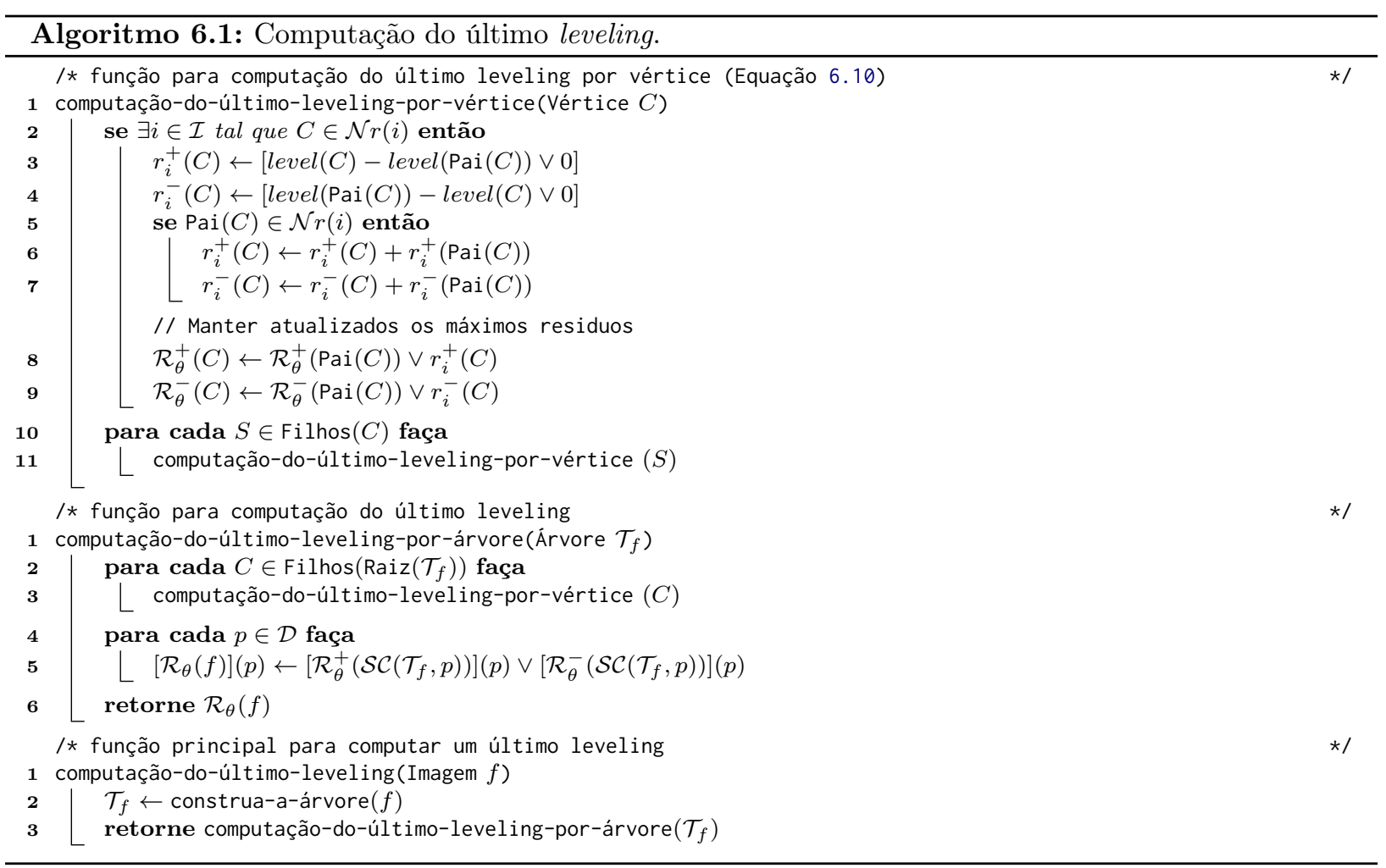

O Algoritmo 6.1 requer uma árvore (max-tree, min-tree ou de formas) de entrada. Então, como apresentado no Capítulo 4, esta árvore pode ser construída por algoritmos baseados em unionfind cuja complexidade computacional é $\mathcal{O}(|\mathcal{D}| \log (|\mathcal{D}|))$. Uma vez que a árvore é construída, a função computação-do-último-leveling-por-árvore do Algoritmo 6.1 pode ser executada, iniciando sua computação da raiz para folhas da árvore (ver linhas 2 e 3). Logo, é executada a função computação-do-último-leveling-por-vértice para cada vértice $C$ da árvore onde é feita a computação dos resíduos no vértice (ver Equação 6.10). Nesta função, é feito um teste na linha 2 para saber se o vértice $C$ está em $\mathcal{N} r(i)$ e este teste é sempre verdadeiro para todos os pontos de podas que resultam em uma primitiva presente na família de primitivas (e pode ser feito em tempo constante), como é discutido no Capítulo 7. Então, quando o teste da linha 2 
é verdadeiro são calculadas (entre as linhas 3 a 7) as Equações 6.10 e Equação 6.11, e nas linhas 8 e 9 são mantidos os máximos resíduos atualizados em $\mathcal{R}_{\theta}^{+}$e $\mathcal{R}_{\theta}^{-}$. Depois, entre as linhas 10 e 11, é propagada a computação para os filhos do vértice $C$ e o processo se repete até as folhas da árvore. Esta função computação-do-último-leveling-por-vértice visita cada vértice somente uma vez e o número de vértice no pior caso é o número de pixels da imagem, portanto sua complexidade computacional é $\mathcal{O}(|\mathcal{D}|)$. Por fim, é reconstruída (nas linhas 4 e 5 da função computação-do-último-leveling-por-árvore) a saída do último leveling. Portanto, como cada vértice é visitado somente uma vez e todas as operações são feitas em tempo constante, então concluímos que a complexidade computacional para construção do último leveling (construção da árvore e do Algoritmo 6.1) é $\mathcal{O}(|\mathcal{D}| \log (|\mathcal{D}|))$.

Na Figura 6.5 é apresentado um exemplo de entrada e saída do Algoritmo 6.1, onde do lado esquerdo está a árvore de entrada min-tree (e também do lado esquerdo, a imagem que produziu a árvore). Note que, a árvore de entrada (do lado esquerdo) está com os respectivos níveis de cinza associados aos vértices, no lado direito está a árvore com os resíduos $r_{i}^{-}$associados aos vértices (calculados pela Equação 6.10). Note ainda que, a família de primitivas utilizada para construir este último leveling está apresentada na Figura 6.4). Além do mais, se um vértice $C$ está colorido em verde, então o teste $\operatorname{Pai}(C) \notin \mathcal{N} r(i)$ é verdeiro e a aresta que conecta $C$ ao vértice Pai $(C)$ é um ponto de poda gerando assim uma primitiva apresentada na Figura 6.4. O resultado final do Algoritmo 6.1 é apresentado na parte inferior do lado direito da Figura 6.5.

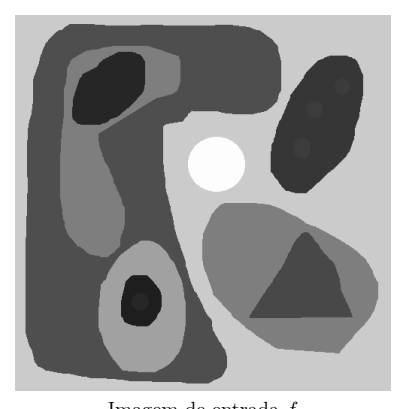

Imagem de entrada $f$

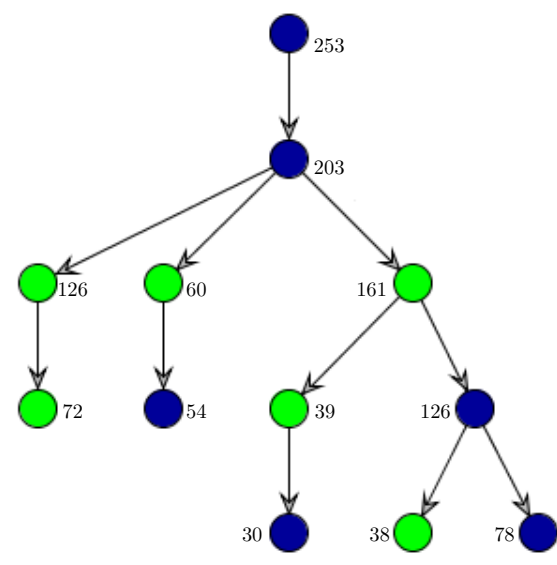

Árvore $\mathcal{T}_{f}$ com os níveis de cinza associado aos vértices

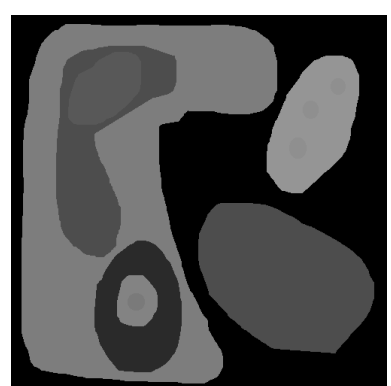

Último leveling negativo $\mathcal{R}_{\theta}^{-}(f)$

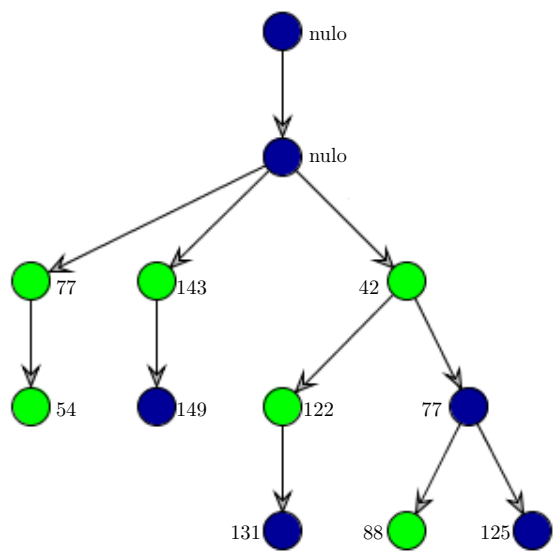

Árvore $\mathcal{T}_{f}$ com os resíduos $r_{i}^{-}$associado aos vértices

Figura 6.5: Exemplo de computação do Algoritmo 6.1 utilizando a árvore da Figura 6.4 como entrada para o algoritmo. Note que, do lado esquerdo está a árvore de entrada com os respectivos níveis de cinza associados aos vértice e do lado direito a árvore com os resíduos associados aos vértices (calculados pela Equação 6.10). Observe também que, se um vértice $C$ está colorido em verde então o teste $\mathrm{Pai}(\mathrm{C}) \notin \mathcal{N} r(i)$ é verdeiro. 


\subsection{Informações associadas aos últimos levelings}

Os últimos levelings são operadores residuais definidos a partir de uma espaço de escala com base em levelings. Na computação de um último leveling analisa-se a evolução do valor residual extraído de duas primitivas consecutivas do espaço de escala e mantém-se os máximos valores residuais (positivo e negativo) para cada pixel. Nesta computação, pode-se associar para cada pixel $p \in \mathcal{D}$, o máximo valor residual a outras informações, na qual podem ser de grande utilidade para realizar uma análise do processo de extração de resíduos ou até mesmo para guiar ou supervisionar o processo de extração de resíduos. Vejamos alguns exemplos de funções associadas:

- A função $q_{\mathcal{I}}: \mathcal{D} \rightarrow \mathcal{I}$, denominada função associada ao índice, que leva um pixel $p \in \mathcal{D}$ ao índice $i \in \mathcal{I}$ do $i$-ésimo resíduo $r_{i}$ que produziu o máximo resíduo não nulo contendo $p$ em $\mathcal{R}_{\theta}(f)$ (mais precisamente, $q(p)$ é o índice $i+1$, assim a função é diferente de zero para $r_{0}$ (Beucher, 2007)), isto é,

$$
\forall p \in \mathcal{D}, q_{\mathcal{I}}(p)= \begin{cases}q_{\mathcal{I}}^{+}(p) & , \text { if }\left[\mathcal{R}_{\theta}^{+}(f)\right](p)>\left[\mathcal{R}_{\theta}^{-}(f)\right](p), \\ q_{\mathcal{I}}^{-}(p) & , \text { caso contrário. }\end{cases}
$$

onde $q_{\mathcal{I}}^{+}(p)=\max \left\{i+1:\left[r_{i}^{+}(f)\right](p)>0\right.$ e $\left.\left[r_{i}^{+}(f)\right](p)=\left[\mathcal{R}_{\theta}^{+}(f)\right](p)\right\}$ e $q_{\mathcal{I}}^{-}(p)=\max \{i+1:$ $\left[r_{i}^{-}(f)\right](p)>0$ e $\left.\left[r_{i}^{-}(f)\right](p)=\left[\mathcal{R}_{\theta}^{-}(f)\right](p)\right\}$.

- A função $q_{\kappa}: \mathcal{D} \rightarrow \mathbb{R}$, denominada função associada ao atributo, que leva um pixel $p \in \mathcal{D}$ ao atributo $\kappa\left(\mathcal{S C}\left(\mathcal{T}_{f}^{i}, p\right)\right)$ extraído da $\mathrm{CC}$ que deu origem ao $i$-ésimo resíduo $r_{i}$ que produziu o máximo resíduo não nulo contendo $p$ em $\mathcal{R}_{\theta}(f)$, ou seja,

$$
\forall p \in \mathcal{D}, q_{\kappa}(p)= \begin{cases}q_{\kappa}^{+}(p) & , \text { if }\left[\mathcal{R}_{\theta}^{+}(f)\right](p)>\left[\mathcal{R}_{\theta}^{-}(f)\right](p), \\ q_{\kappa}^{-}(p) & , \text { caso contrário. }\end{cases}
$$

onde $q_{\kappa}^{+}(p)=\max \left\{\kappa\left(\mathcal{S C}\left(\mathcal{T}_{f}^{i}, p\right)\right):\left[r_{i}^{+}(f)\right](p)>0\right.$ e $\left.\left[r_{i}^{+}(f)\right](p)=\left[\mathcal{R}_{\theta}^{+}(f)\right](p)\right\}$ e $q_{\kappa}^{-}(p)=$ $\max \left\{\kappa\left(\mathcal{S C}\left(\mathcal{T}_{f}^{i}, p\right)\right):\left[r_{i}^{-}(f)\right](p)>0\right.$ e $\left.\left[r_{i}^{-}(f)\right](p)=\left[\mathcal{R}_{\theta}^{-}(f)\right](p)\right\}$.

As computações dessas funções associadas podem ser realizadas modificando o Algoritmo 6.1 nos pontos ondes são calculados os resíduos $r_{i}^{+}$e $r_{i}^{-}$para incluir, logo após os cálculos dos resíduos $r_{i}^{+}$ e $r_{i}^{-}$, a implementação das funções associadas, como pode-se observar a partir das linhas em azul do Algoritmo 6.2. Assim, no teste da linha 5, da função computação-do-último-leveling-por-vértice, é verificado se o pai do vértice $C$ pertence a mesma primitiva que $C$ e, se for verdadeiro, é feita a soma da diferença com o vértice pai, neste caso (ver linhas entre 8 e 11), a primitiva que produziram os máximos resíduos para os pixels de $C$ é a mesma do vértice Pai $(C)$. Mas, se o teste da linha 5 for falso, então os resíduos $r_{i}^{+}$e $r_{i}^{-}$são de uma nova primitiva e, neste caso, é verificado entre as linhas 13 e 24 se a nova primitiva produziu máximos resíduos e assim atualiza o índice e o atributo que geraram máximos resíduos. Por fim, é reconstruída (entre as linhas 4 e 13 da função computação-do-último-leveling-por-árvore) a saída do último leveling e as imagens associadas aos índices e os atributos que produziram os máximos resíduos não nulos. Na Figura 7.1 é apresentado um exemplo de entrada e saída do Algoritmo 6.2, onde da esquerda para direita está a imagem de entrada e a árvore min-tree com os respectivos níveis de cinza associados aos vértices, a imagem do último levelings e a árvore min-tree com os máximos resíduos associados aos vértices, a imagem 
rotulada dos índices que produziram os máximos resíduos e a árvore min-tree com os índices associados aos vértices, a imagem do atributo (altitude) obtida a partir dos vértices que produziram os máximos resíduos e a árvore min-tree com os valores da altitude associadas aos vértices.

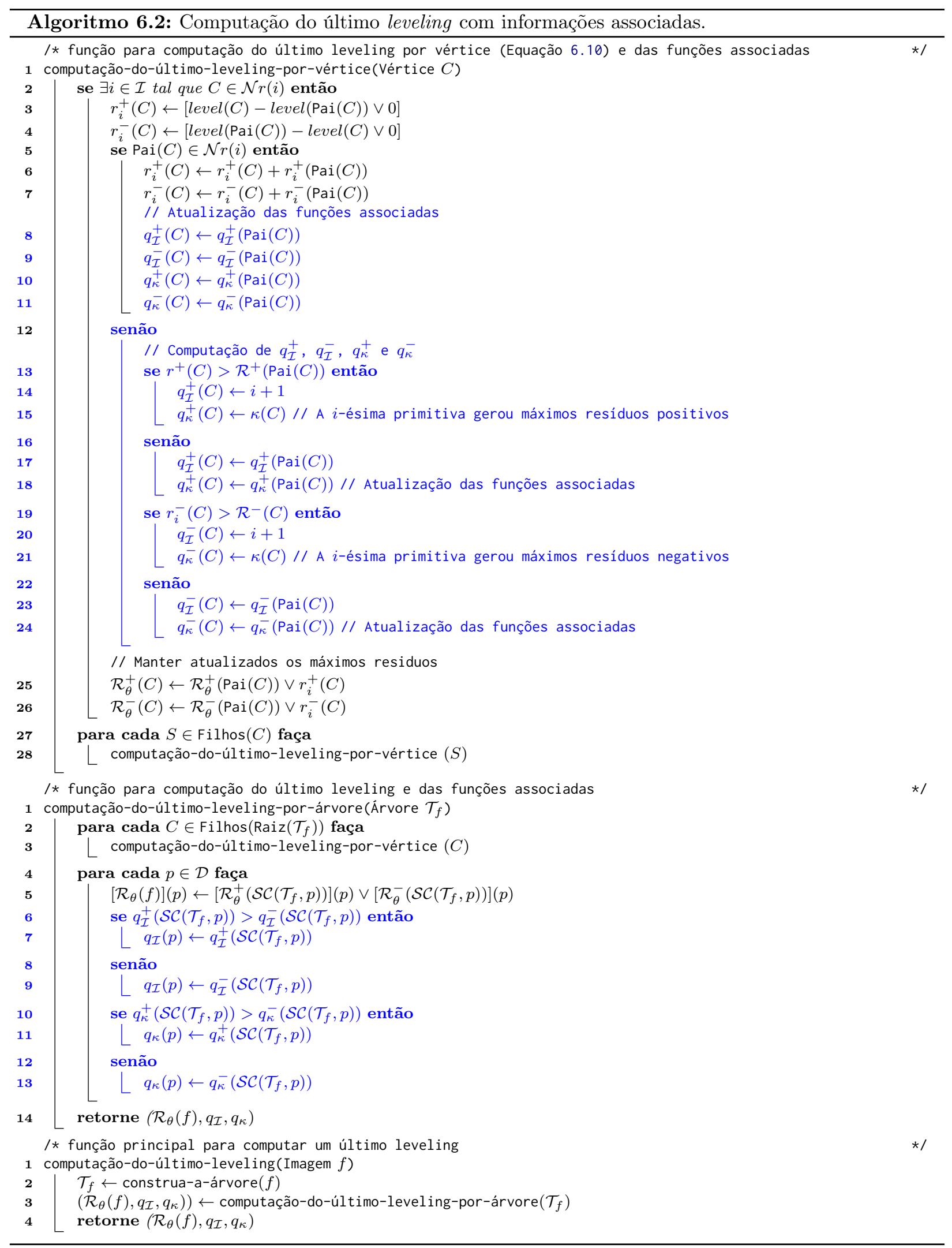

Na Figura 6.7 são apresentados exemplos de operadores últimos levelings onde a família de primitivas são: aberturas por atributo de altura, fechamento por atributo de altura e últimos filtros 
por grãos utilizando atributo de altura.
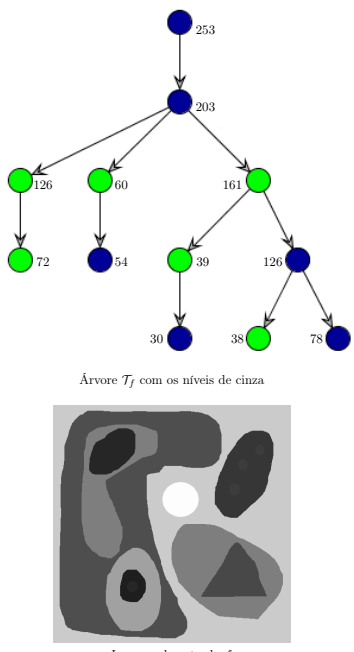
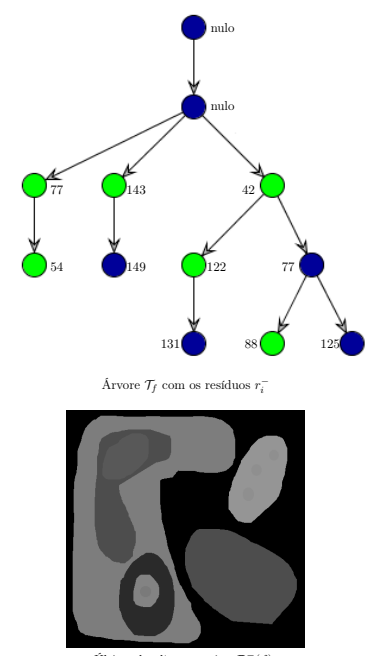
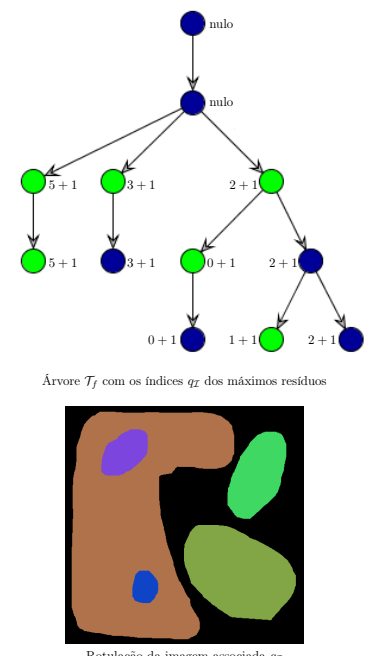
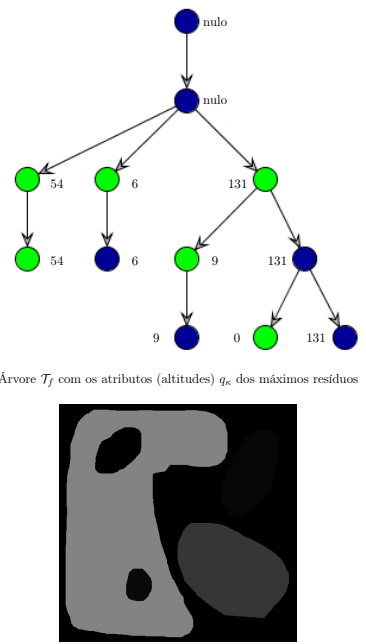

Figura 6.6: Exemplo de computação de último leveling com funções associadas utilizando uma árvore mintree.

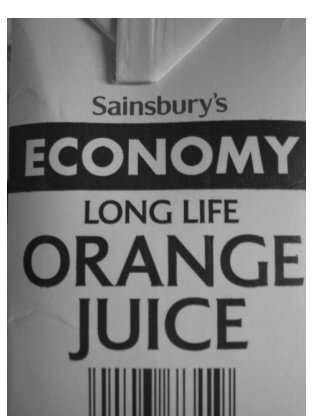

imagem de entrada

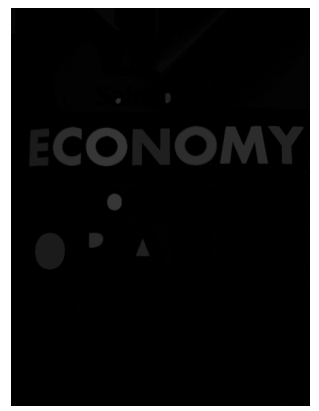

UAO

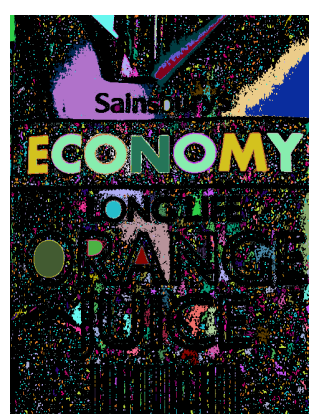

rotulação da imagem associada ao UAO

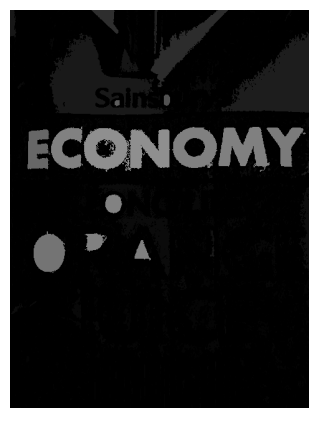

imagem associada (altitude) ao UAO

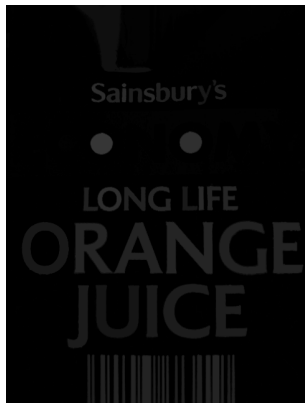

UAC

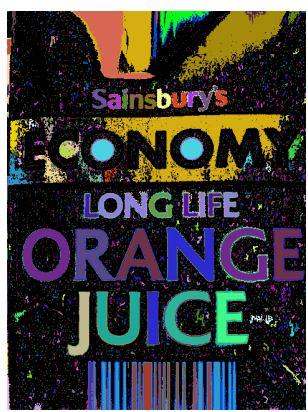

otulação da imagem associada ao UAC

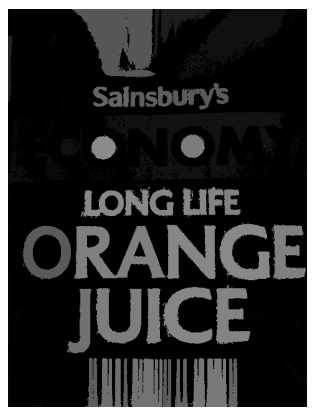

imagem associada (altitude) ao UAC

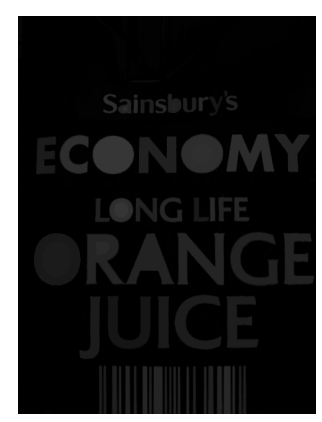

UGF

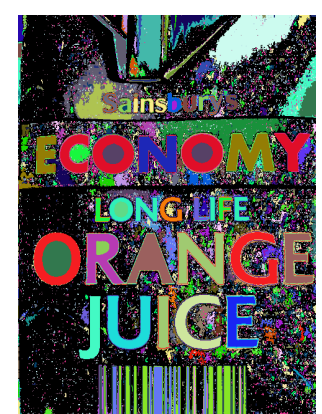

rotulação da imagem associada ao UGF

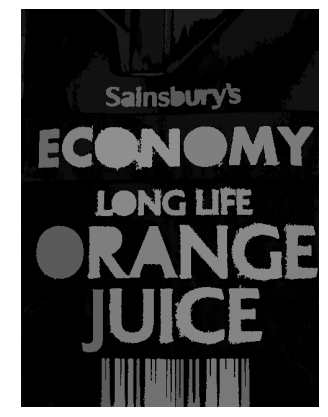

imagem associada (altitude) ao UG

Figura 6.7: Exemplos de aplicações de últimos levelings: (da esquerda para direita) imagem de entrada, $U A O, U A C$ e $U G F$. 


\title{
Estratégias para construções de últimos
} levelings

\begin{abstract}
Resumo do capítulo
Neste capítulo são apresentadas estratégias para construções de últimos levelings. Em particular, são apresentadas estratégias para escolha de famílias de primitivas. Dessa forma, um último leveling é o supremo dos i-ésimos resíduos $r_{i}$ extraídos ao longo da família de primitiva escolhida. Neste processo de extração dos $i$-ésimos resíduos $r_{i}$ podem existir resíduos extraídos de regiões indesejáveis. Assim, são apresentados neste capítulo estratégias para filtrar resíduos $r_{i}$ indesejáveis extraídos ao longo da família de primitivas, e assim o último leveling é definido como supremo dos resíduos $r_{i}$ desejáveis.
\end{abstract}

\subsection{Estratégias para escolher a família de primitivas}

A escolha da família de primitivas leva em consideração hipóteses sobre as regiões que se pretende extrair os valores residuais de uma imagem e sobre essas hipóteses, constrói-se um espaço de escala contendo as primitivas mais significativas para construção do último leveling. Neste sentido, um ponto de partida para a escolha da família de primitivas é a questão da polaridade dos objetos de interesses, ou seja, se os objetos são claros ou escuros em relação ao seu background. Neste caso, as primitivas podem ser obtidas por reconstruções inferiores ou superiores o que fazem produzirem resíduos positivos $\mathcal{R}^{+}$(objetos claros) ou negativos $\mathcal{R}^{-}$(objetos escuros). Mas, se os objetos de interesses estiverem em ambas polaridades então as primitivas podem ser obtidas por reconstruções de formas ou alternativamente, pode-se considerar simultaneamente duas famílias de primitivas obtidas por reconstruções inferiores e superiores e depois combinar seus resultados.

O resultado de um operador último leveling é diretamente influenciado pela escolha da família de primitivas as quais são obtidas, como já mencionado, por reconstruções de podas sucessivas de uma árvore. Embora no Algoritmo 6.1, aparece somente o conjunto $\mathcal{N} r(i)$ que contém vértices obtidos pela diferenças entre duas podas consecutivas. Assim, para cada índice $i \in \mathcal{I}$ de uma família de primitivas, precisa-se determinar o conjunto $\mathcal{N} r(i)$. Esta tarefa é simples, mas depende das primitivas incluídas na família de primitivas.

A ideia para determinar a família de primitivas (ver Algoritmo 7.1) consiste em fazer marcações dos vértices $C$ da árvore, por meio de um critério $\Gamma: \mathcal{P}(\mathcal{D}) \rightarrow$ verdadeiro, falso\}, de tal forma que a remoção da aresta que conecta um vértice $C$ ao vértice $\operatorname{Pai}(C)$ resulta na árvore podada. Assim, estas marcações em uma árvore $\mathcal{T}_{f}$ dão origem as árvores $\left(\mathcal{T}_{f}^{0}, \mathcal{T}_{f}^{1}, \ldots, \mathcal{T}_{f}^{\mathcal{I}_{M A X}}\right)$ obtidas por 
podas sucessivas e consequentemente os conjuntos $\mathcal{N} r(i)$ de vértices removidos entre duas árvores consecutivas. Estas marcações podem ser obtidas (e consecutivamente, cada árvore $\mathcal{T}_{f}^{i}$ e seu conjunto $\mathcal{N} r(i))$, por exemplo, através de um percurso em pós-ordem em $\mathcal{T}_{f}$. Mas é claro, podem ser usados outros tipos de ordenação para visitar os vértices de $\mathcal{T}_{f}$, entretanto isto não modifica o resultado dos últimos levelings graças à Proposição 6.15. Na Figura 7.1 são mostrados dois exemplos de construção da família de primitivas usando o mesmo critério $\Gamma$, um pelo Algoritmo 7.1 onde as primitivas são produzidas de acordo com o percurso em pós-ordem e a outra família de primitivas estão ordenadas pelo atributo de altura.
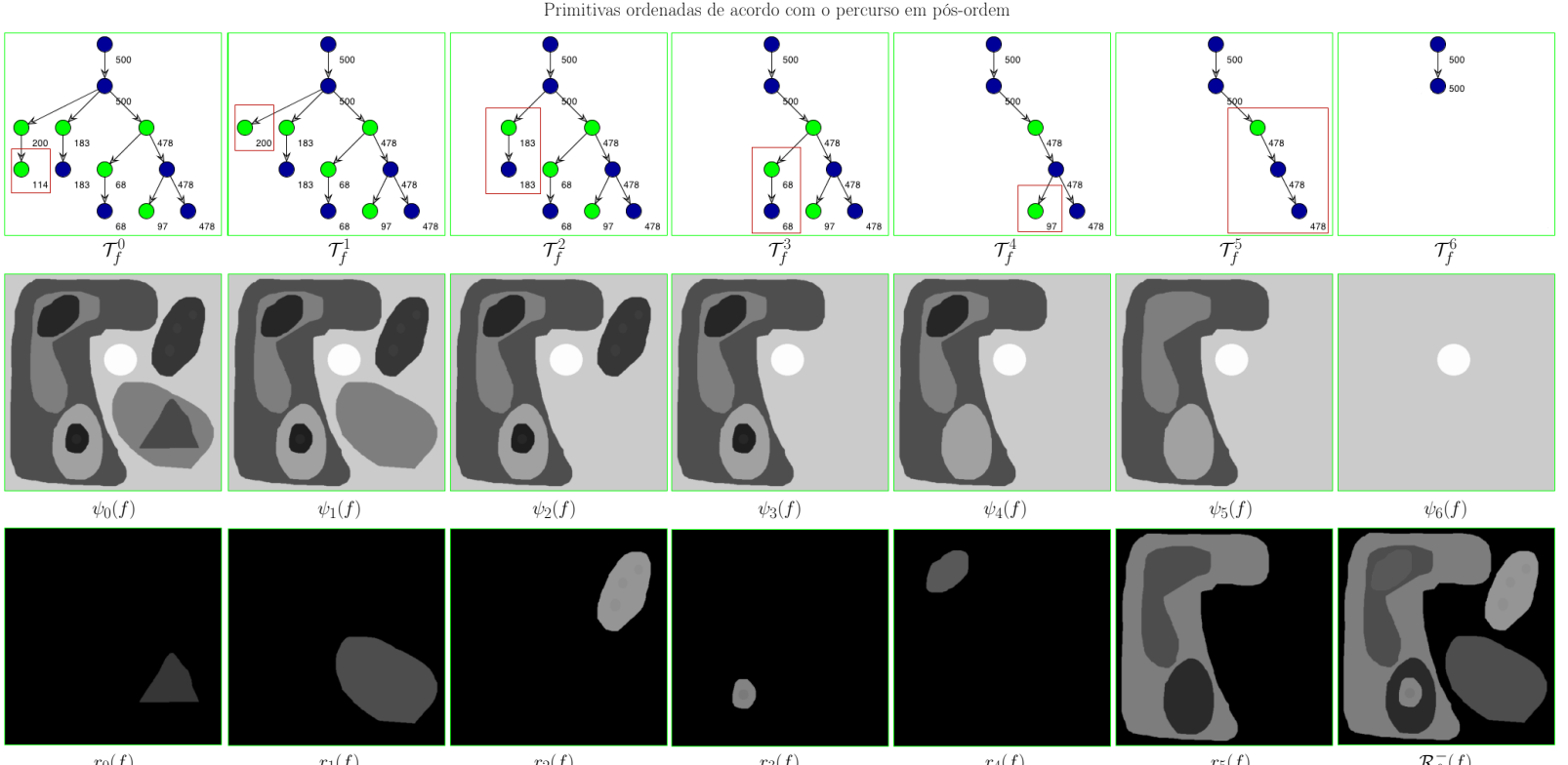

$\psi_{2}(f)$
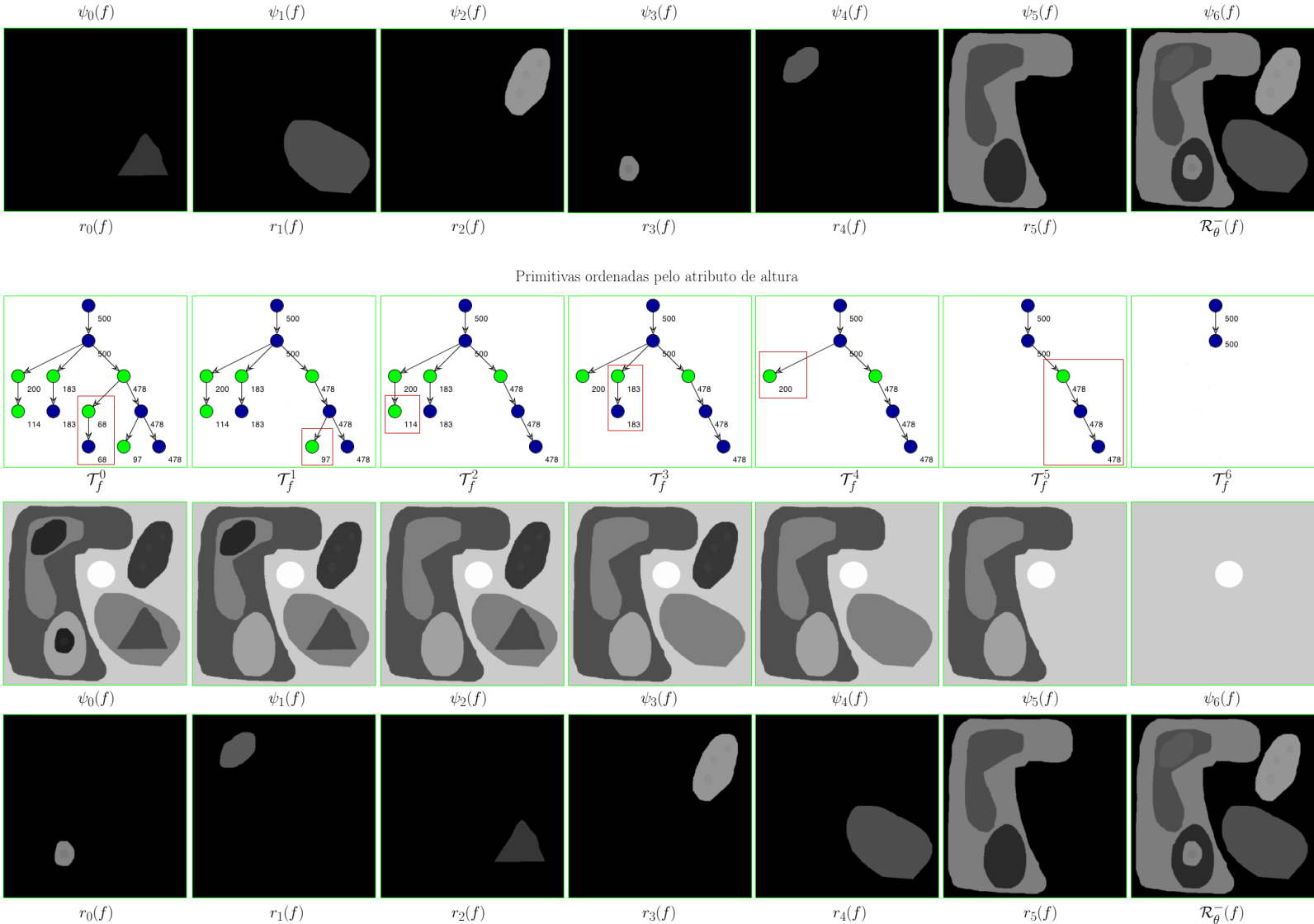

Figura 7.1: Exemplo de computação de um último leveling negativo onde é evidenciado que a ordem das marcações nos vértices da árvore não modifica o resultado final dos últimos levelings.

Por exemplo, o conjunto com maior número de primitivas que podem ser derivadas de uma árvore $\mathcal{T}_{f}$ é dado pelo seguinte critério: um vértice $C$ de uma árvore $\mathcal{T}_{f}$ satisfaz a condição do 


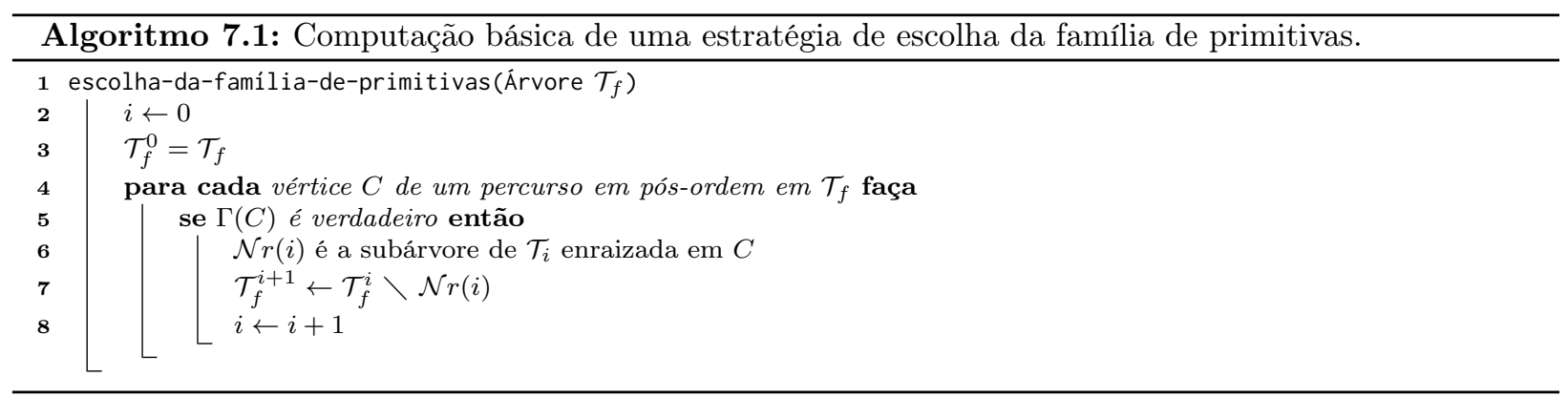

critério $\Gamma$ se, e somente se, $C$ não é a raiz de $\mathcal{T}_{f}$, ou seja,

$$
\Gamma(C) \text { é verdadeiro } \Longleftrightarrow C \neq \operatorname{Raiz}\left(\mathcal{T}_{f}\right)
$$

Assim, pode-se extrair todas as possíveis árvores $\left(\mathcal{T}_{f}^{0}, \mathcal{T}_{f}^{1}, \ldots, \mathcal{T}_{f}^{\mathcal{I}_{M A X}}\right)$ de $\mathcal{T}_{f}$ e consequentemente a família de primitivas $\left(\psi_{0}(f)=\operatorname{Rec}\left(\mathcal{T}_{f}^{0}\right), \psi_{1}(f)=\operatorname{Rec}\left(\mathcal{T}_{f}^{1}\right), \ldots, \psi_{f}^{\mathcal{I}_{M A X}}=\operatorname{Rec}\left(\mathcal{T}_{\mathcal{I}_{M A X}}\right)\right)$ e então extrair os $i$-ésimos resíduos positivos e negativos. Mas isso não parece ser uma boa ideia se o propósito é extrair o contraste de uma imagem, pois os valores residuais gerados entre duas primitivas consecutivas são próximos de zero. Isso ocorre por causa da grande quantidade de níveis de cinza presente em uma imagem e também pela hierarquia dos conjuntos de níveis. Por outro lado, se o propósito envolve reconhecimento de objetos pode ser uma boa ideia usar todas as primitivas possíveis. Isto leva às seções seguintes onde são definidas algumas estratégias para construir famílias de primitivas que podem gerar valores residuais mais significativos para os últimos levelings.

\subsubsection{Primitivas baseadas em atributos}

Como apresentado no Capítulo 5, filtrar uma imagem usando um atributo pode ser feito simplesmente podando os vértices da árvore que satisfaz um certo critério, como por exemplo, os vértices cujo o valor do atributo é menor que um dado limiar. Assim, muitos diferentes tipos de informações podem ser armazenadas em cada vértice da árvore. Estas informações são chamadas de atributos, mais precisamente, um atributo é uma função $\kappa$ que representa uma informação armazenada no vértice da árvore como: área, volume, diâmetro, circularidade, entre outros. Então, reconstruções de árvores podadas com atributos crescentes são levelings. Assim, um conjunto crescente de limiares $\left(t_{1}, t_{2}, \ldots, t_{\mathcal{I}_{M A X}}\right)$ com $t_{1}<t_{2}<\ldots<t_{\mathcal{I}_{M A X}}$ dá origem a um espaço de escala baseado em levelings.

Então, famílias de primitivas baseadas em aberturas por atributos, fechamentos por atributos e filtros por grãos podem ser determinadas de um conjunto de limiares derivados da própria árvore $\mathcal{T}_{f}$, isto é, $T_{\kappa}=\left\{\kappa(C): C\right.$ é um vértice de $\mathcal{T}_{f}$ e $\left.\kappa(C) \leq t_{\mathcal{I}_{M A X}}\right\}$. Então, a partir do conjunto de limiares $T_{\kappa}$ pode-se estabelecer o critério $\Gamma$ para todo vértice $C$ de $\mathcal{T}_{f}$ seguinte forma:

$$
\Gamma(C) \text { é verdadeiro } \Longleftrightarrow \exists t_{j} \in T_{\kappa} \text { satisfazendo } \kappa(C)=t_{j} \text { e } \kappa(\operatorname{Pai}(C))>t_{j} \text {. }
$$

Note que, quando $\Gamma(C)$ é verdadeiro, então $C$ está em $\mathcal{T}_{f}^{j-1}$ mas não está em $\mathcal{T}_{f}^{j}$, pois os vértices da $j$-ésima árvore podada tem atributos maiores que $t_{j}$ (ver Seção 5.3.1). Logo, $C \in \mathcal{N} r(j-1)$. Note também que, para este conjunto de limiares $T_{\kappa}$, o critério: $\Gamma(C)$ é verdadeiro, é equivalente a, 
$\kappa(C) \neq \kappa(\operatorname{Pai}(C))$ e $\kappa(C) \leq t_{\mathcal{I}_{M A X}}$ pois,

$$
\begin{aligned}
& \Gamma(C) \text { é verdadeiro } \\
& \Longleftrightarrow \exists t_{j} \in T_{\kappa} \text { satisfazendo } \kappa(C)=t_{j} \text { e } \kappa(\operatorname{Pai}(C))>t_{j} \\
& \Longleftrightarrow \kappa(C) \neq \kappa(\operatorname{Pai}(C)) \text { e } \kappa(C) \leq t_{\mathcal{I}_{M A X}} .
\end{aligned}
$$

Os valores residuais de um último leveling negativo (respectivamente, positivo) $\mathcal{R}_{\theta}^{-}$(respectivamente, $\mathcal{R}_{\theta}^{+}$) são as maiores diferenças entre duas primitivas consecutivas de uma família de primitivas. Neste sentido, na Figura 7.2 é mostrado um exemplo com duas aplicações de últimos levelings negativos $\mathcal{R}^{-}$em uma dada imagem $f \in \mathcal{F}(\mathcal{D})$ onde se analisa a evolução do valor residual para um dado pixel ao longo da computação de $\mathcal{R}^{-}(f)$, usando duas famílias de primitivas diferentes: (1) fechamento por atributo de área e (2) fechamento por atributo de altura do retângulo envolvente. Observe que, o contraste de um objeto é dividido em diversas transições e a maior destas transições é o valor residual obtido por $\mathcal{R}^{-}(f)$, que inevitavelmente é menor do que o verdadeiro contraste existente entre o objeto e o seu plano de fundo. Assim, as famílias de primitivas por atributos de área e volume tendem a simplificar a imagem muito lentamente, uma vez que existem muitas primitivas distintas que podem ser extraídas com estes atributos. Por um outro lado, este efeito pode ser reduzido utilizando tipos de atributos que resultam em um mesmo valor para diferentes vértices, como por exemplo: altura, largura e diâmetro do retângulo envolvente. Mesmo assim, o contraste real ainda é subestimado.

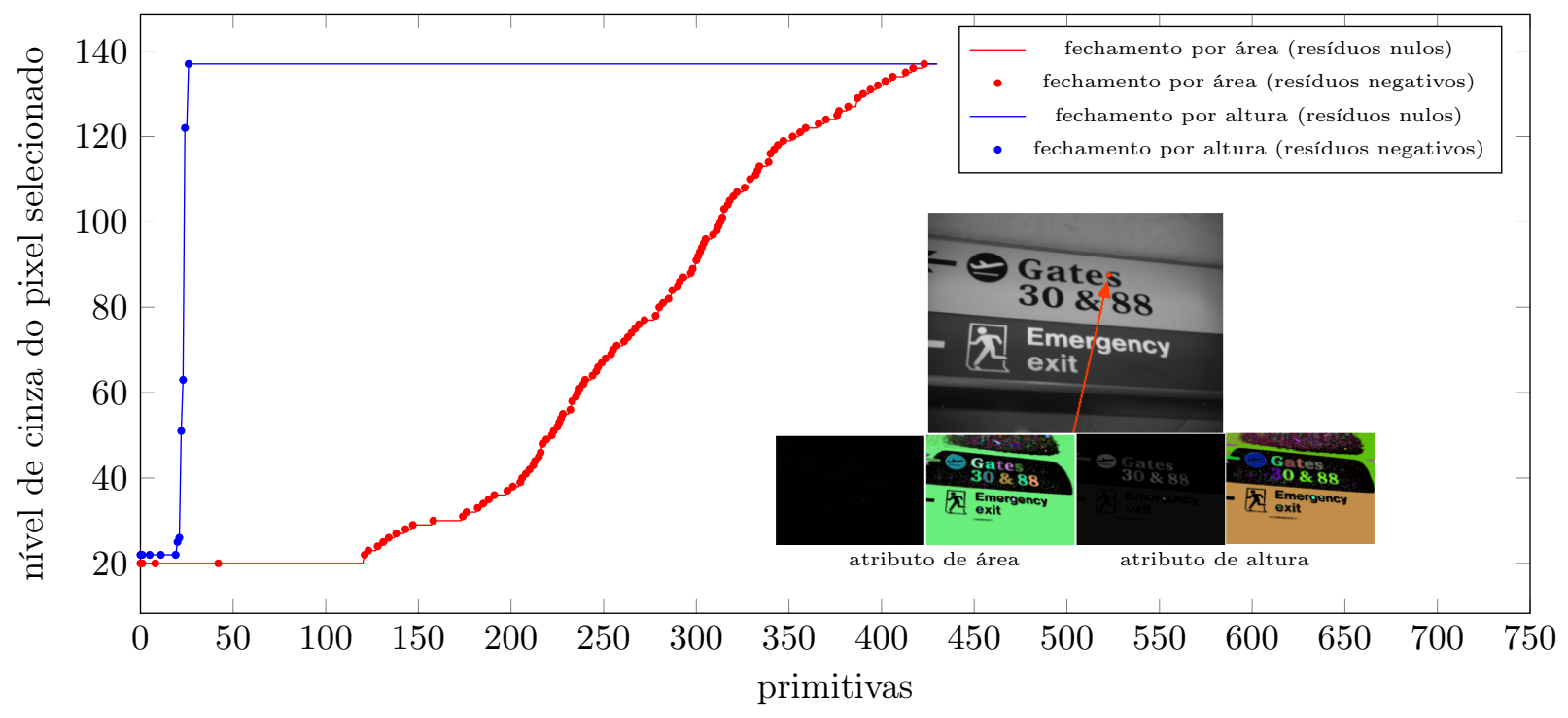

Figura 7.2: Análise da evolução residual para o pixel marcado de vermelho da imagem de entrada.

Com base nestas considerações, Marcotegui et al. (2011), propôs uma solução chamada transição gradual que precisa de um limiar para ajustar o contraste. Neste sentido, é proposta uma segunda solução baseada em valores de extinção que não faz uso de limiares.

- Primitivas baseadas em transições graduais

Como mostrado anteriormente, as famílias de aberturas por atributos, fechamentos por atributos e filtros por grãos podem ser determinadas a partir de um conjunto crescente de limiares $\left(t_{1}, t_{2}, \ldots, t_{\mathcal{I}_{M A X}}\right)$ para um atributo crescente $\kappa$. Assim, um vértice $C \in \mathcal{N} r(i) \subseteq \mathcal{T}_{i}$ satisfaz as 
condições do critério $\Gamma$, se

$$
\begin{aligned}
& \Gamma(C) \text { é verdadeiro } \\
& \Longleftrightarrow \exists t_{j} \in T_{\kappa} \text { satisfazendo } \kappa(C)=t_{j} \text { e } \kappa(\operatorname{Pai}(C))>t_{j} \\
& \Longleftrightarrow \kappa(C) \neq \kappa(\operatorname{Pai}(C)) \text { e } \kappa(C) \leq t_{\mathcal{I}_{M A X}} \\
& \Longleftrightarrow \kappa(\operatorname{Pai}(C))-\kappa(C)>0 \text { e } \kappa(C) \leq t_{\mathcal{I}_{M A X}} .
\end{aligned}
$$

Então, uma maneira de reduzir o desconforto de subestimar o contraste é selecionar os vértices (para serem as referências das podas) que tenham uma grande variação $\Delta$ do valor do atributo em relação ao seu vértice pai, isto é,

$$
\Gamma(C) \text { é verdadeiro } \Longleftrightarrow \kappa(\operatorname{Pai}(C))-\kappa(C)>\Delta \text { e } \kappa(C) \leq t_{\mathcal{I}_{M A X}}
$$

Assim, quando $\Delta=0$, tem-se as tradicionais famílias de primitivas utilizando os limiares do conjunto $T_{\kappa} ; \Delta=1$ é acumulada uma série de resíduos não nulos; $\Delta=2$ é acumulada uma série de resíduos separados por pelo menos dois resíduos não nulos e assim por diante. Assim, inclui-se em $\mathcal{N} r(i)$, vértices com pequenas variações $\Delta$ nos valores dos seus atributos.

O inconveniente dessa abordagem é encontrar o valor para $\Delta$, uma vez que esta informação é global para todos os vértices da árvore e muitas vezes não é possível estimar o real contraste para todas as regiões da imagem como pode ser observado na Figura 7.3.

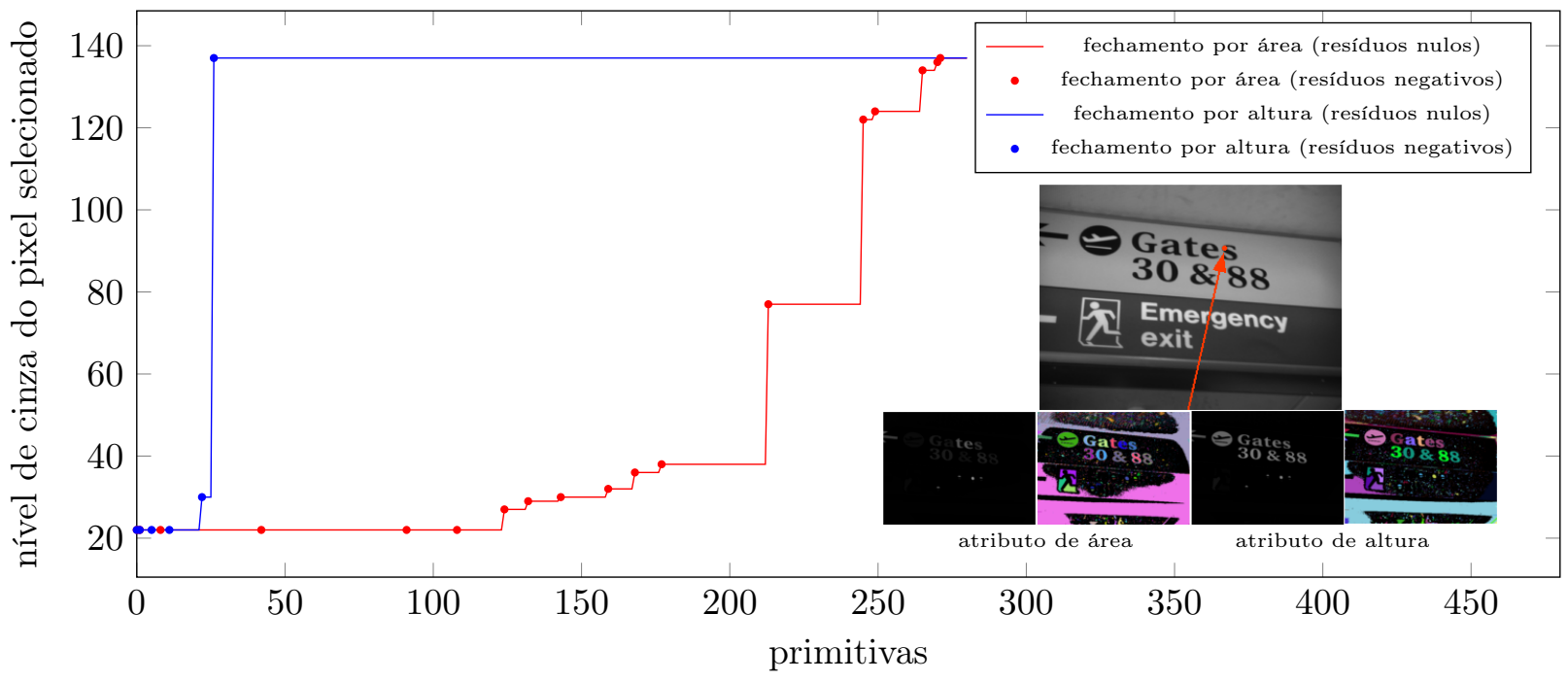

Figura 7.3: Análise da evolução residual para o pixel marcado de vermelho da imagem de entrada.

- Primitivas baseadas em valores de extinção

Uma outra maneira de reduzir o desconforto de subestimar o contraste dos objetos é por meio do uso de valores de extinção (Grimaud, 1992; Vachier e Meyer, 1995). Os valores de extinções são medidas de persistências extraídas das extremas (minimas ou máximas) regionais. Em poucas palavras, o valor de extinção de uma extrema regional $\mathcal{M}$ para um atributo crescente $\kappa$ é o valor máximo de um filtro por atributos, tal que esta extrema regional ainda exista após a filtragem. Assim, são marcados somente vértices nos quais os valores do seus atributos correspondem a valores de extinções para a extrema regional presente no vértice (Grimaud, 1992; Vachier e Meyer, 1995). 
Este procedimento pode ser computado por um algoritmo com base em árvore de componentes (Silva e de Alencar Lotufo, 2011) e adaptado no Algoritmo 7.2 para selecionar vértices baseada no valor de extinção, da seguinte forma: para cada vértice folha $L$ da árvore $\mathcal{T}_{f}$ (isto é, regional extrema), um caminho em direção ao vértice raiz é iniciado (entre as linhas 2 e 3 ). Quando um vértice $N_{L}$ com mais de um filho aparece (ou seja, uma bifurcação), no caminho, um verificação é feita em cada filho $C_{L}$ de $N_{L}$. Se o vértice $C_{L}$ tem um irmão $C \in \operatorname{Filhos}\left(N_{L}\right)$ tal que $\kappa(C)>\kappa\left(C_{L}\right)$, tem-se que o valor do atributo do vértice $C_{L}$ no caminho desta folha é definido como o valor de extinção para folha $L$ (entre as linhas 4 a 6 ). Neste caso,

$$
\Gamma(C) \text { é verdadeiro } \Longleftrightarrow C \in \operatorname{Filhos}\left(N_{L}\right) \text { e } \kappa(C) \leq t_{\mathcal{I}_{\text {MAX }}}
$$

onde $t_{\mathcal{I}_{M A X}}$ é o valor máximo para um atributo da família de primitiva, em seguida, a próxima folha é analisada. Caso contrário, continua-se no caminho até encontrar a próxima bifurcação. Finalmente, se o caminho atingir a raiz, então o valor de extinção para folha $L$ é o valor do primeiro vértice antes de chegar com maior valor de atributo.

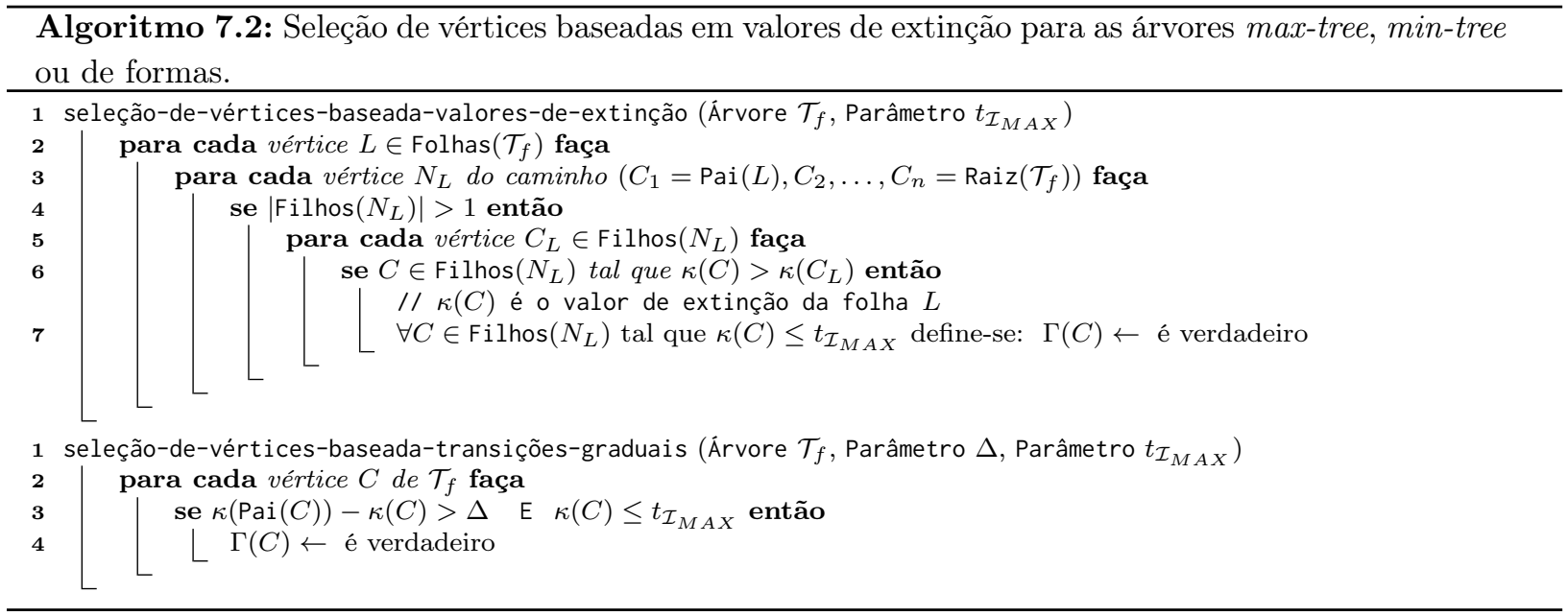

Note que os vértices que correspondem as pequenas transições graduais de níveis de cinza ao longo da borda não são selecionados. Na Figura 7.4 é reapresentada a evolução residual usando duas famílias de primitivas baseada em valores de extinções.

Na Figura 7.5 são apresentados exemplos de entrada e saída para as seleções dos vértices a serem podados por estratégias de escolha de famílias de primitivas com base em (1) atributo de área, (2) transições graduais com parâmetro $\Delta=90$ para o atributo de área e (3) valores de extinção usando o atributo de área.

\subsubsection{Primitivas baseadas em imagens marcadoras}

Como apresentado na Seção 5.3.2, um espaço de escala pode ser derivado a partir de uma família de imagens marcadoras $\left(g_{1}, g_{2}, \ldots, g_{I_{M A X}}\right)$. Dessa forma, conforme apresentado na Seção 5.3.2, deriva-se da versão estendida de uma árvore $\mathcal{T}_{f}$ uma sequência de árvores podadas sucessivamente $\left(\mathcal{T}_{f}^{0}, \mathcal{T}_{f}^{1}, \ldots, \mathcal{T}_{f}^{\mathcal{I}_{M A X}}\right)$ por meio da família de marcadores. Assim, um vértice $C$ da versão estendida 


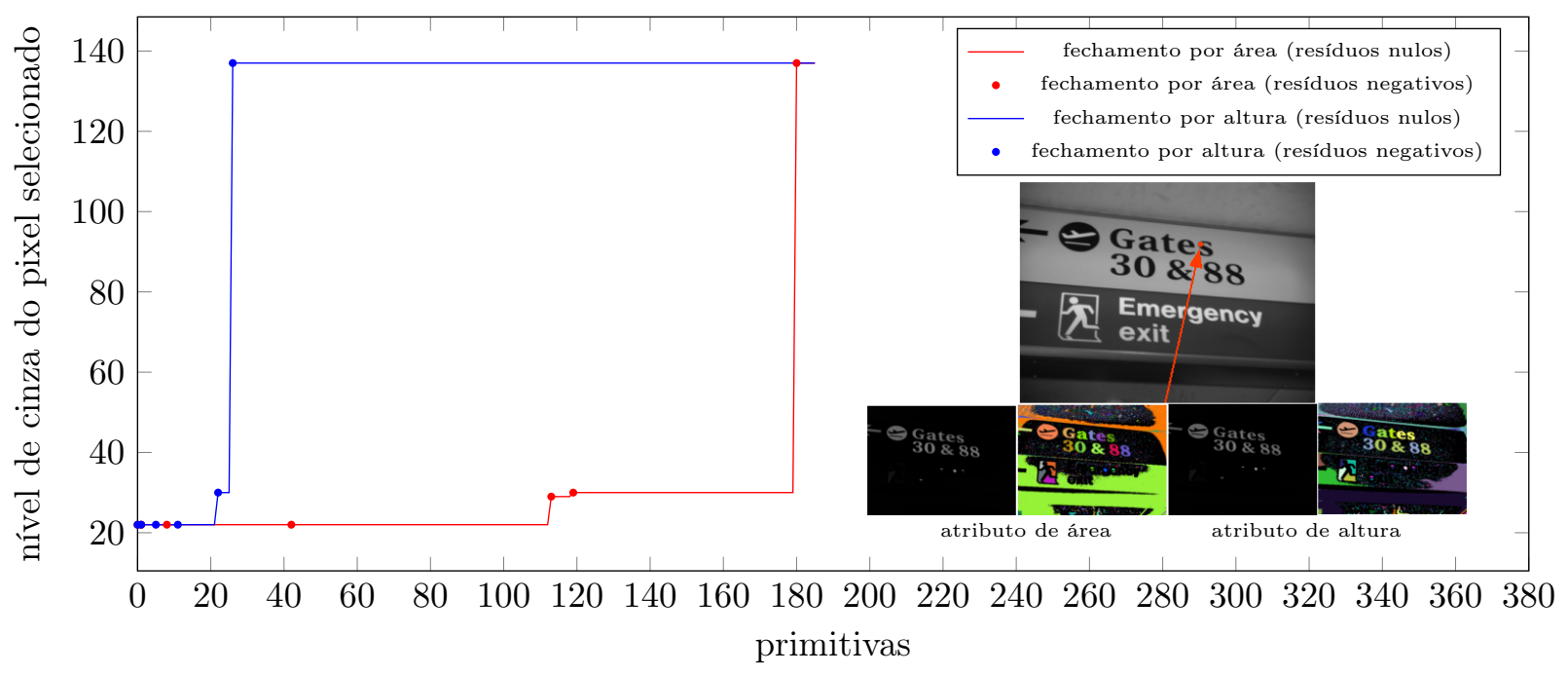

Figura 7.4: Análise da evolução residual para o pixel marcado de vermelho da imagem de entrada.

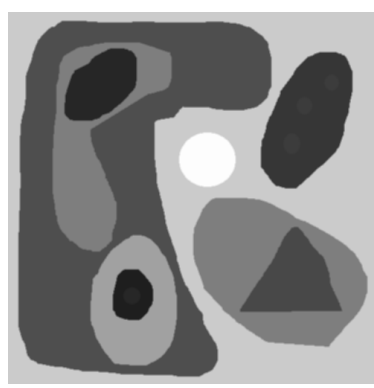

imagem de entrada (suavizada)

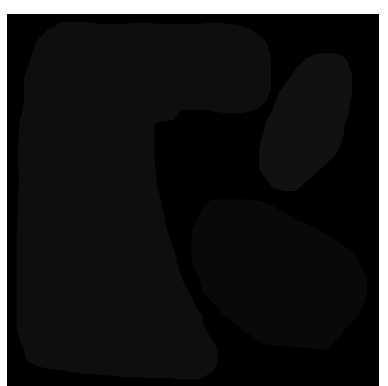

UAC com atributo de área

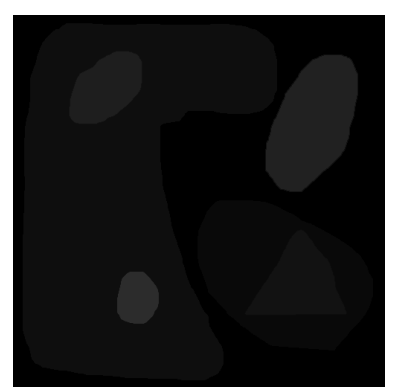

UAC com transições graduais

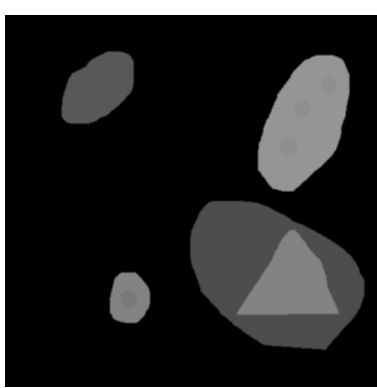

UAC com valores de extinção

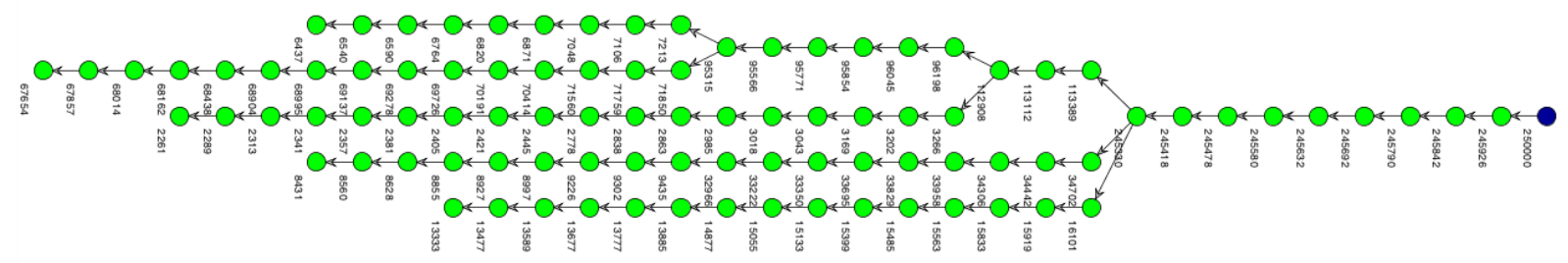

Árvore da imagem de entrada (podas baseadas em atributo de área)

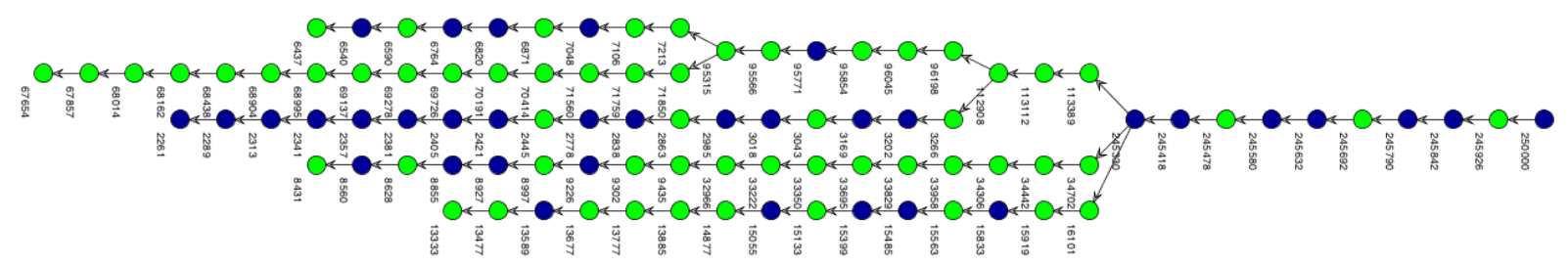

Árvore da imagem de entrada (podas baseadas transições graduais de valor $\Delta=90$ para o atributo de área)

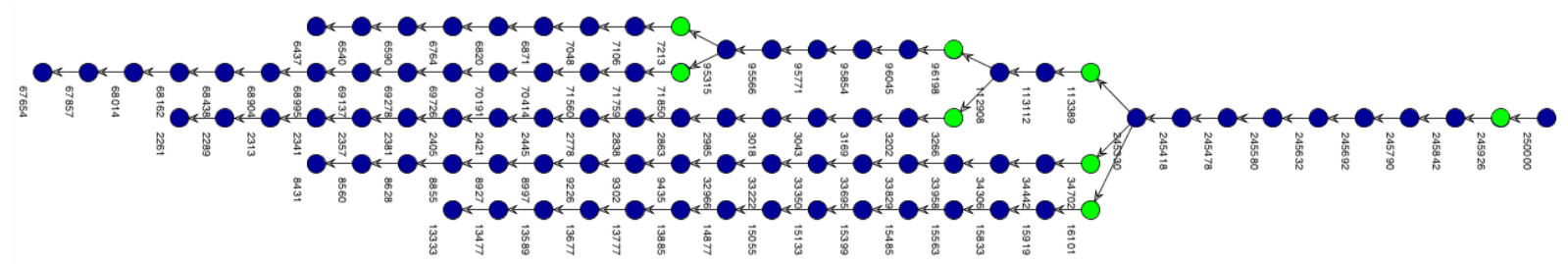

Figura 7.5: Exemplos de entrada e saída dos processamentos baseados em transições graduais e valores de extinção.

de $\mathcal{T}_{f}$ é selecionado pelo critério $\Gamma$ se,

$\Gamma(C)$ é verdadeiro $\Longleftrightarrow \exists j$ tal que o vértice $C$ foi removido e

Pai $(C)$ foi preservado na operação de poda que produziu $\mathcal{T}_{f}^{j}$. 
O uso de família de primitivas construídas usando imagens marcadoras pode prover mais liberdade ao operador, pois é possível adicionar informações a priori na construção da família de marcadores. Mas o uso dessas primitivas aumenta substancialmente o custo computacional dos últimos levelings. Por este motivo, sempre recomendamos o uso de primitivas baseadas em atributos e, se houver informações à priori, então pode-se aplicá-las através de estratégias de filtragens dos valores residuais indesejáveis (ver Seção 7.2).

\subsection{Estratégias para filtrar resíduos indesejáveis}

Os últimos levelings são operadores definidos como o supremo de resíduos consecutivos extraídos ao longo de uma família de primitivas. Durante este processo de extração residual é comum a extração de valores residuais de regiões indesejáveis da imagem. Por conta disso, muitas vezes esses valores residuais indesejáveis se sobrepõem aos valores residuais extraídos de regiões desejáveis, devido a própria concepção dos últimos levelings que consideram sempre os máximos valores residuais extraídos ao longo da família de primitivas. Esse problema de concepção dos últimos levelings, é chamado por Retornaz (2007) como miopia, que é normalmente produzido por estruturas aninhadas ou por transições graduais. Nesta direção, Hernandez e Marcotegui (2011) propuseram uma solução para este problema usando informação de formas no processo de extração residual, mas o inconveniente dessa abordagem é que os valores residuais podem ser modificados devido uma função de similaridade que multiplica com os valores residuais e com isso as propriedade dos últimos levelings acabam sendo anuladas.

Por outro lado, nesta tese propõe-se preservar os valores residuais extraídos ao longo da família de primitivas e informações sobre regiões indesejáveis são adicionadas por meio de um processo de filtragem. Assim, o resultado do último leveling é melhorado quando se filtram os resíduos $r_{i}$ extraídos de regiões indesejáveis, como pode ser observado na Figura 7.6, a qual exemplifica o cálculo do último leveling somente dos $i$-ésimos resíduos $r_{i}$ que satisfazem um critério de filtragem $\Omega: \mathcal{P}(\mathcal{D}) \rightarrow$ \{verdadeiro, falso $\}$.

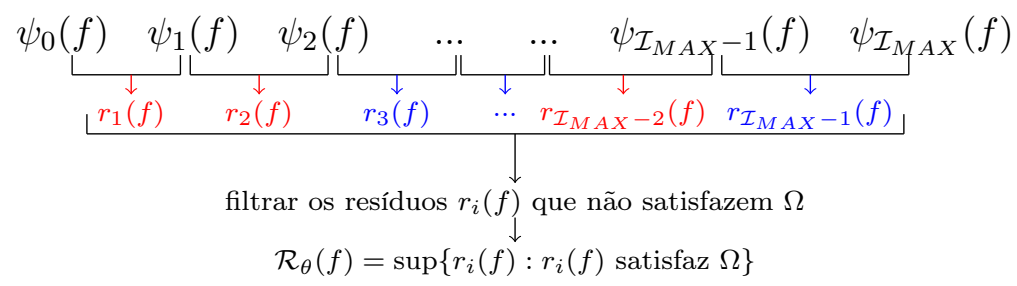

Figura 7.6: Esquema para filtrar os i-ésimos resíduos $r_{i}$ extraídos de regiões indesejáveis.

Este procedimento de filtragem pode ser implementado de diversas maneiras, mas todas elas partem do mesmo princípio. Para decidir se um resíduo $r_{i}^{+}(f)$ (respectivamente, $r_{i}^{-}(f)$ ) é filtrado ou não filtrado, basta verificar se algum vértice $C \in \mathcal{N} r(i)$ satisfaz o critério de filtragem e assim os $i$-ésimos resíduos $r_{i}^{\Omega+}(f)$ e $r_{i}^{\Omega-}(f)$ podem ser redefinidos com o critério de filtragem $\Omega$ por

$$
\left[r_{i}^{\Omega+}(f)\right](p)= \begin{cases}r_{\mathcal{T}_{f}^{i}}^{+}\left(\mathcal{S C}\left(\mathcal{T}_{f}^{i}, p\right)\right), & \text { se } \mathcal{S C}\left(\mathcal{T}_{f}^{i}, p\right) \in \mathcal{N} r(i) \text { e } \exists C \in \mathcal{N} r(i), \Omega(C) \text { é falso } \\ 0, & \text { caso contrário, }\end{cases}
$$




$$
\left[r_{i}^{\Omega-}(f)\right](p)= \begin{cases}r_{\mathcal{T}_{f}^{i}}^{-}\left(\mathcal{S C}\left(\mathcal{T}_{f}^{i}, p\right)\right), & \text { se } \mathcal{S C}\left(\mathcal{T}_{f}^{i}, p\right) \in \mathcal{N} r(i) \text { e } \exists C \in \mathcal{N} r(i), \Omega(C) \text { é falso } \\ 0, & \text { caso contrário, }\end{cases}
$$

e consequentemente, os últimos levelings com critério de filtragem $\Omega$ podem ser redefinidos como

$$
\mathcal{R}_{\Omega}^{+}(f)=\sup _{i \in \mathcal{I}}\left\{r_{i}^{\Omega+}(f)\right\}, \quad \mathcal{R}_{\Omega}^{-}(f)=\sup _{i \in \mathcal{I}}\left\{r_{i}^{\Omega-}(f)\right\} \quad \text { e } \quad \mathcal{R}_{\Omega}(f)=\mathcal{R}_{\Omega}^{+}(f) \vee \mathcal{R}_{\Omega}^{-}(f)
$$

A seguir, são apresentadas duas estratégias de filtragens de resíduos indesejáveis. Uma com base em um vetor de atributos (Urbach et al., 2007, 2005) similar ao trabalho de Hernandez e Marcotegui (2011) e a outra baseada em regiões estáveis (Matas et al., 2004).

\subsubsection{Filtragem com base em vetor de atributos}

O reconhecimento de formas é um dos problemas fundamentais na área de análise de imagens e diversas abordagens têm sido propostas ao longo dos últimos anos. Um dos subproblemas de reconhecimento de formas, denominado problema de decisão (Veltkamp e Hagedoorn, 2000), consiste em comparar dois padrões $A$ e $B$ com uma medida de similaridade $d$ por meio de um dado limiar $\alpha$, ou seja, decidir se similaridade é maior do que o limiar. No contexto desta tese, os padrões são vetores de atributos extraídos dos vértices das árvores ou dos CCs dos conjunto de níveis (que podem estar saturados). Então, a medida de similaridade $d$ é uma função definida como segue:

Definição 7.1 (Medida de similaridade (Veltkamp, 2001)). Uma medida de similaridade entre os padrões $A$ e $B$ em $\mathcal{P}(\mathcal{D})$, é definida por uma função $d: \mathcal{P}(\mathcal{D}) \times \mathcal{P}(\mathcal{D}) \rightarrow[0,1] \in \mathbb{R}$ verificando as seguinte condições:

1. Identidade: $d(A, A)=1$;

2. Unicidade: $d(A, B)=1$ implica em $A=B$;

3. Simétrica: $d(A, B)=d(B, A)$.

Assim, pretende-se construir um classificador $\Omega$ para decidir os rótulos (filtrar ou não filtrar) para os vértice de $\mathcal{N} r(i)$, de acordo com um determinado critério construído por uma medida de similaridade entre a forma representada por um vértice e uma forma de um padrão de referência $\Omega_{\text {ref }} \in \mathcal{P}(\mathcal{D})$. Desse modo, o classificador é construído para todo vértice $C \in \mathcal{P}(\mathcal{D})$ da seguinte forma:

$$
\Omega(C)= \begin{cases}\text { filtrar, } & \text { se } d\left(C, \Omega_{r e f}\right) \leq \alpha, \\ \text { não filtrar, } & \text { caso contrário, }\end{cases}
$$

Uma forma simples de construir $\Omega$ é por meio dos tradicionais descritores sobre a geometria do CC (área, largura, altura, perímetro, etc) e as suas relações (circularidade, retangularidade, momentos, etc). Por exemplo:

- Classificar vértices com formatos retangulares

$$
\Omega_{\text {retangularidade }}(C)= \begin{cases}\text { não filtrar, }, & , \text { se } \frac{\text { Área }(C)}{\operatorname{Largura}_{\theta}(C) \times \operatorname{Altura}_{\theta}(C)} \leq \alpha, \\ \text { filtrar, } & , \text { caso contrário },\end{cases}
$$


onde Área $(C)$ é a área de $C, \operatorname{Largura}_{\theta}(C)$ e $\operatorname{Altura}_{\theta}(C)$ são as medidas de largura e a altura do retângulo envolvente sobre $C$ rotacionado na direção do maior eixo.

- Classificar vértices com formatos circulares

$$
\Omega_{\text {circularidade }}(C)= \begin{cases}\text { não filtrar, } & , \text { se } 4 \pi \frac{\text { Área }(C)}{\text { Perímetro }(C)^{2}} \leq \alpha, \\ \text { filtrar, } & \text {, caso contrário, }\end{cases}
$$

onde Área $(C)$ é a área do CC $C$ e Perímetro $(C)$ é o perímetro do $\mathrm{CC} C$.

- Classificar vértices com formatos de estruturas alongadas.

$$
\Omega_{\text {alongamento }}(C)= \begin{cases}\text { não filtrar, } & , \text { se } \frac{\text { Área }(C)}{\pi \times \text { Maior eixo }(C)^{2}} \leq \alpha, \\ \text { filtrar, } & \text {, caso contrário. }\end{cases}
$$

onde Área $(C)$ é a área do CC $C$ e Maior eixo $(C)$ é o comprimento do maior eixo de $C$ (Xu et al. , 2013).

Além disso, outros descritores de formas mais complexos, tais como shape context, casamento de template, modelos deformáveis, entre outros podem ser utilizados. Apesar de todas estas possibilidades para filtrar resíduos indesejáveis durante o processo de extração de resíduos. No entanto, é necessário ter cuidado com a escolha destes descritores para preservar as propriedades de invariâncias (translação, rotação e escala) dos últimos levelings. Além disso, dependendo da aplicação pode ser que o tempo computacional seja relevante, neste caso, é melhor dar preferência aos descritores computáveis incrementalmente na árvore (Matas e Zimmermann, 2005). Estes descritores referem-se aos atributos que podem ser computados por meio dos atributos dos seus filhos e o conjunto de pixels canônicos presentes nos vértices. Em outras palavras, não é necessário reconstruir o CC representado pelo vértice para fazer a computação do atributo. Um procedimento simples (ver Algoritmo 7.3), para fazer esta computação é por meio de um percurso em profundidade, em que ao visitar um vértice em pré-ordem pode-se inicializar o seu atributo e em uma visita em pós-ordem pode-se atualizar o seu atributo com os atributos dos seus filhos já computados anteriormente.

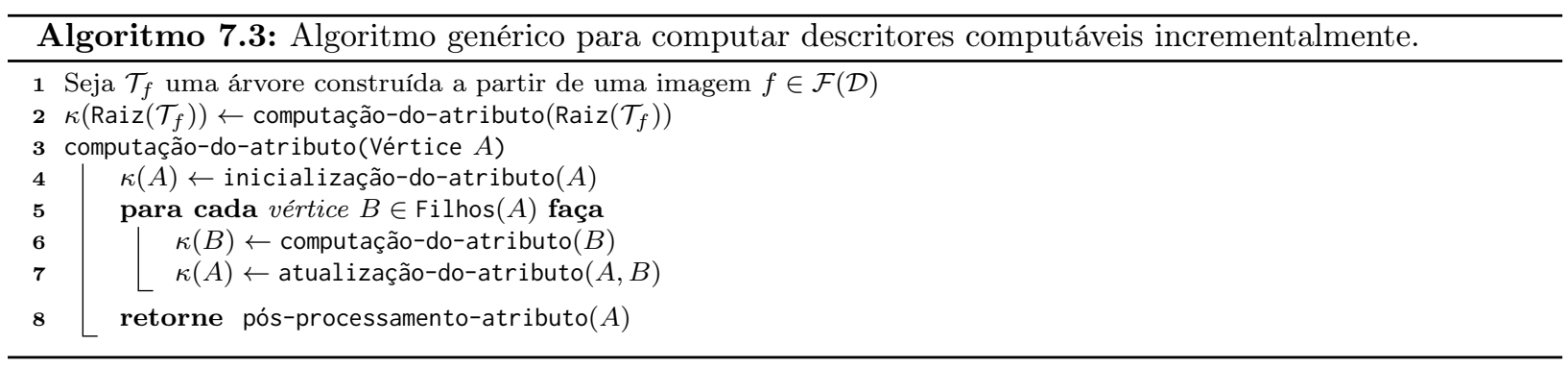

Assim, atributo como área, volume, altura, largura, perímetro, variância, momentos, entre outros, podem ser computados incrementalmente por meio do Algoritmo 7.3. Um exemplo simples de implementação das funções inicialização-do-atributo e atualização-do-atributo para o atributo área é apresentado no Algoritmo 7.4 e, de maneira semelhante, podem ser construídas as funções para os outros atributos (Najman e Couprie, 2006; Neumann e Matas, 2012; Silva, 2009; Urbach e Wilkinson, 2002; Urbach et al., 2007, 2005; Xu, 2013). 


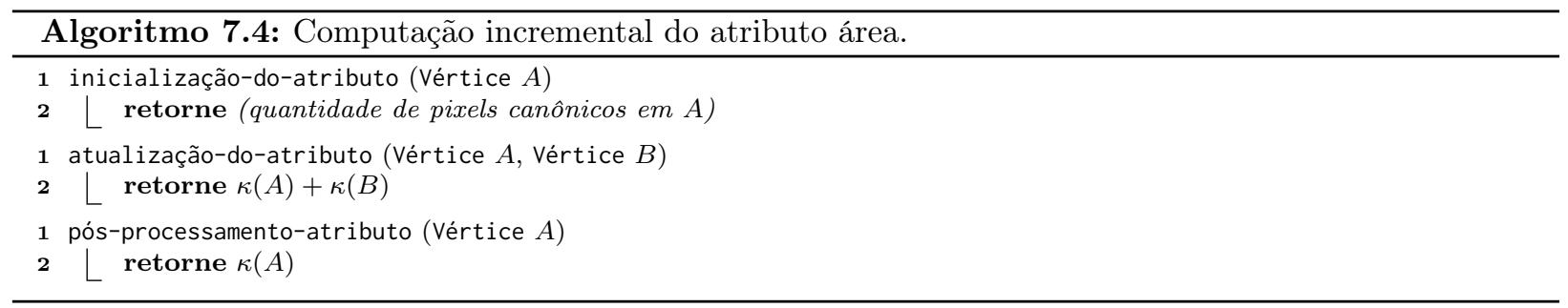

Nas Figuras 7.7 e 7.8 são mostrados exemplos de aplicações dos últimos levelings com intuito de segmentar objetos circulares e com estruturas finas por meio de filtragem dos resíduos indesejáveis.

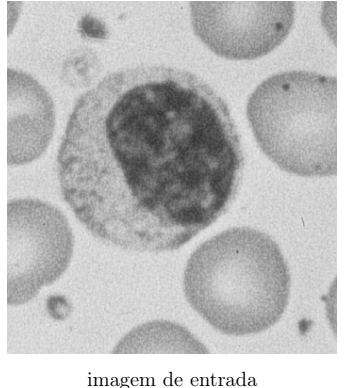

imagem de entrada

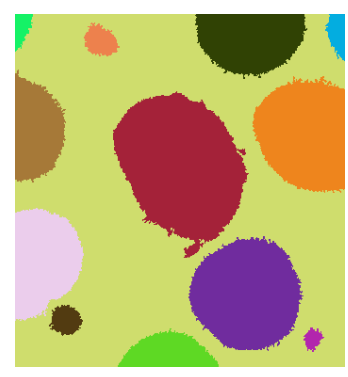

rotulação da imagem associada ao UGF com filtro de circularidade

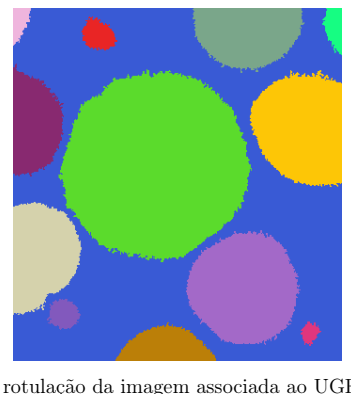

com filtro de circularidade

Figura 7.7: Aplicações dos últimos filtros por grãos utilizando famílias de primitivas baseadas em valores de extinções com atributo de área e filtragens baseadas em circularidades.

No exemplo apresentado na Figura 7.7 foram utilizadas famílias de primitivas baseadas em valores de extinções com atributo de área e filtragens baseadas em circularidades.

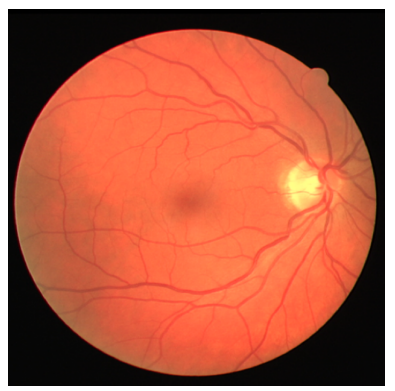

(a) imagem de entrada RGB

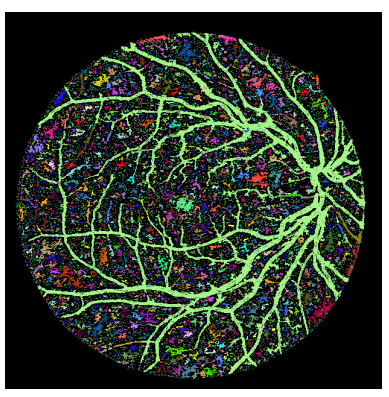

(d) rotulação da imagem associada ao UAO

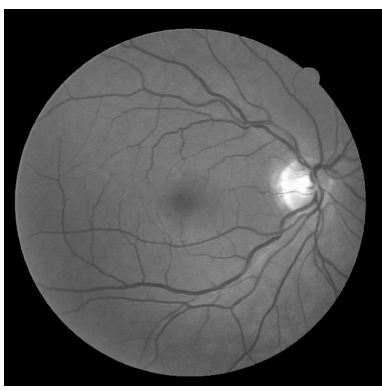

(b) imagem de entrada (banda G)

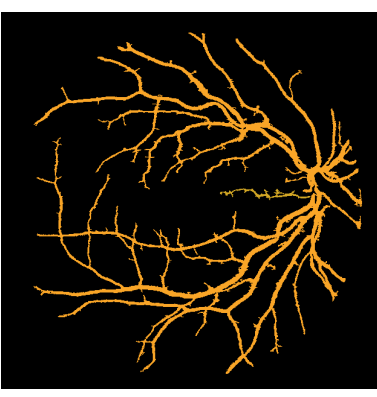

(e) rotulação da imagem associada ao UAO com filtro de alongamento

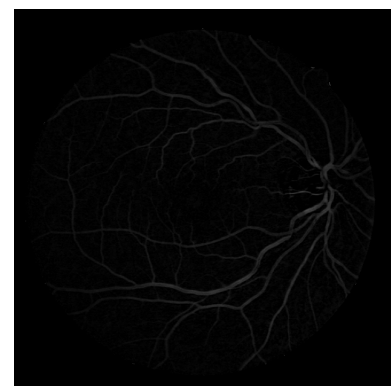

(c) fechamento top-hat

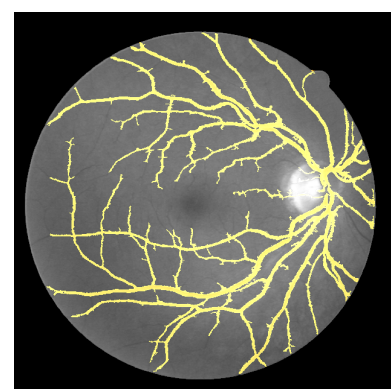

(f) Sobreposição de (b) com (e)

Figura 7.8: Aplicação da última abertura com informação de formas de estruturas finas.

Neste segundo exemplo apresentado na Figura 7.8 também foi utilizada uma família de primitivas baseada em valor de extinção com atributo de área e utilizando filtragem com base no afinamento. 


\subsubsection{Filtragem com base em regiões estáveis}

Nesta seção apresentamos um outro modelo de filtragem de resíduos indesejáveis por meio de um detector de regiões chamado Regiões Extremais Maximamente Estáveis (MSER - do inglês, Maximally Stable Extremal Regions) introduzido por Matas et al. (2004). Eles são amplamente utilizados em uma grande variedade de aplicações em visão computacional, incluindo correspondência entre imagens (Kimmel et al., 2011; Matas et al., 2004), recuperação de imagens (Nister e Stewenius , 2006; Sivic e Zisserman, 2003), reconhecimento de objetos (Obdrzalek e Matas, 2005), rastreamento de objetos (Donoser e Bischof, 2006), entre outros. As regiões detectadas por este método são CCs dos conjuntos de níveis de estabilidade máxima e, como mostrado por Kimmel et al. (2011), são invariante as transformações afins o que é uma vantagem quando comparados com os detectores SIFT (Kimmel et al., 2011).

A estabilidade é uma função que associa os CCs $C_{\lambda}$ extraídos de um conjunto de nível de limiar $\lambda$ com um valor de estabilidade definido por uma relação entre intensidade (limiar do conjunto de nível) e os limites exteriores do CC. Mais precisamente, a estabilidade de um CC $C_{\lambda}$ é a razão entre a diferença das áreas de dois CCs comparáveis a $C_{\lambda}$ de uma certa intensidade, com a área de $C_{\lambda}$, ou seja,

$$
\operatorname{Estabilidade}\left(C_{\lambda}, \Delta\right)=\frac{\operatorname{Área}\left(C_{\lambda}-\Delta\right)-\operatorname{Área}\left(C_{\lambda}+\Delta\right)}{\operatorname{Área}\left(C_{\lambda}\right)}
$$

onde $\Delta \in \mathbb{K}$ é um parâmetro de estabilidade e $C_{\lambda-\Delta}, C_{\lambda}$ e $C_{\lambda+\Delta}$ são CCs obtidos por conjuntos de níveis de limiares $\lambda-\Delta, \lambda+\Delta$ e $\lambda$ satisfazendo ou $C_{\lambda-\Delta} \subset C_{\lambda} \subset C_{\lambda+\Delta}$, ou $C_{\lambda-\Delta} \supset C_{\lambda} \supset C_{\lambda+\Delta}$.

Considere uma sequência de $\operatorname{CCs}\left(C_{0}, C_{1}, \ldots, C_{K}\right)$ extraídos de conjuntos de níveis de limiares de valores $0,1, \ldots, K$ de tal forma que $C_{0} \subset C_{1} \subset \ldots \subset C_{K}$. As regiões de estabilidades máximas são os pontos de mínimos locais para função Estabilidade definida para uma sequência de CCs $\left(C_{1}, C_{2}, \ldots, C_{n}\right)$ e são chamadas de MSER. No trabalho de Matas et al. (2004), são definidos outros dois conjuntos de CCs MSER mais específicos, os MSER+ definidos usando somente CCs dos conjuntos de níveis superiores e os MSER- definidos usando somente CCs dos conjunto de níveis inferiores. As detecções dessas regiões podem ser computadas eficientemente por meio de uma árvore, por exemplo, com a max-tree extraí as regiões MSER+, com a min-tree as regiões MSER-, com a árvore de formas as regiões MSER.

A computação dos vértices MSER pode ser feita para cada sequência de vértices $\left(C_{1}, C_{2}, \ldots, C_{n}\right)$ definida por um intervalo $\left[F, \operatorname{Raiz}\left(\mathcal{T}_{f}\right)\right]_{\mathcal{T}_{f}}$ de uma folha $F \in \operatorname{Folhas}\left(\mathcal{T}_{f}\right)$ para a raiz da árvore. Assim, pode-se visitar os vértices $\left(C_{1}, C_{2}, \ldots, C_{n}\right)$ nesta ordem por meio de um caminho partindo da folha $F$ em direção a raiz da árvore. A cada vértice $C_{i}$ vistado do caminho pode-se calcular a função de estabilidade. Quando fazemos este procedimento para todos os possíveis intervalos da folha a raiz, muitos vértices acabam sendo revisitados. Então, uma outra abordagem é armazenar para cada vértice $C$ da árvore, um ancestral $A$ e um descendente de maior área $D$ de $C$ tal que

$$
\text { ou level }(D)=\operatorname{level}(C)-\Delta \text { e level }(A)=\operatorname{level}(C)+\Delta
$$

se $C$ é um CC originado de um conjunto de nível superior,

$$
\text { ou level }(D)=\operatorname{level}(C)+\Delta \text { e level }(A)=\operatorname{level}(C)-\Delta
$$


se $C$ é um CC originado de um conjunto de nível inferior. Assim,

$$
\operatorname{Estabilidade}(C, \Delta)=\frac{\text { Área }(D)-\text { Área }(A)}{\text { Área }(C)}
$$

e consequentemente $C$ é um vértice MSER se, e somente se,

$$
\operatorname{Estabilidade}(D, \Delta)<\operatorname{Estabilidade}(C, \Delta)<\operatorname{Estabilidade}(A, \Delta) \text {. }
$$

Essa ideia leva ao Algoritmo 7.5 para computação dos vértices MSER de uma árvore max-tree, mintree ou de formas. Primeiramente, são armazenados em duas funções Ascendente e Descendente os vértices ascendentes e descendeste para cada vértice $C$ da árvore $\mathcal{T}_{f}$. Durante esta computação (ver linhas 2 a 7 da função extração-dos-vértices-MSER) armazena-se o ancestral do vértice $C$ que só existe um e mantém-se atualizado o descendente de maior área do vértice $C$. Note que, se um vértice $A$ é ancestral de $C$, então o vértice $C$ é descendente do vértice $A$. Uma vez computadas as funções Ascendente e Descendente, então calcula-se a função de estabilidade para todos os vértices (entre as linhas 8 a 10). Em seguida, para cada vértice $C$ da árvore é verificado se ele é um vértice MSER (linhas 11 e 12). Esse algoritmo, no pior caso, visita para cada vértice $\Delta$ vezes para produzir as funções Ascendente e Descendente. Depois, são visitados mais duas vezes os vértices da árvore para computar a função de estabilidade e a máxima estabilidade. Portanto, a complexidade do algoritmo no pior caso é $\mathcal{O}\left(\left|\mathcal{T}_{f}\right| \times \Delta\right)$, onde $\left|\mathcal{T}_{f}\right|$ é a quantidade de vértices da árvore $\mathcal{T}_{f}$ que normalmente é inferior a quantidade de pixels de $f$.

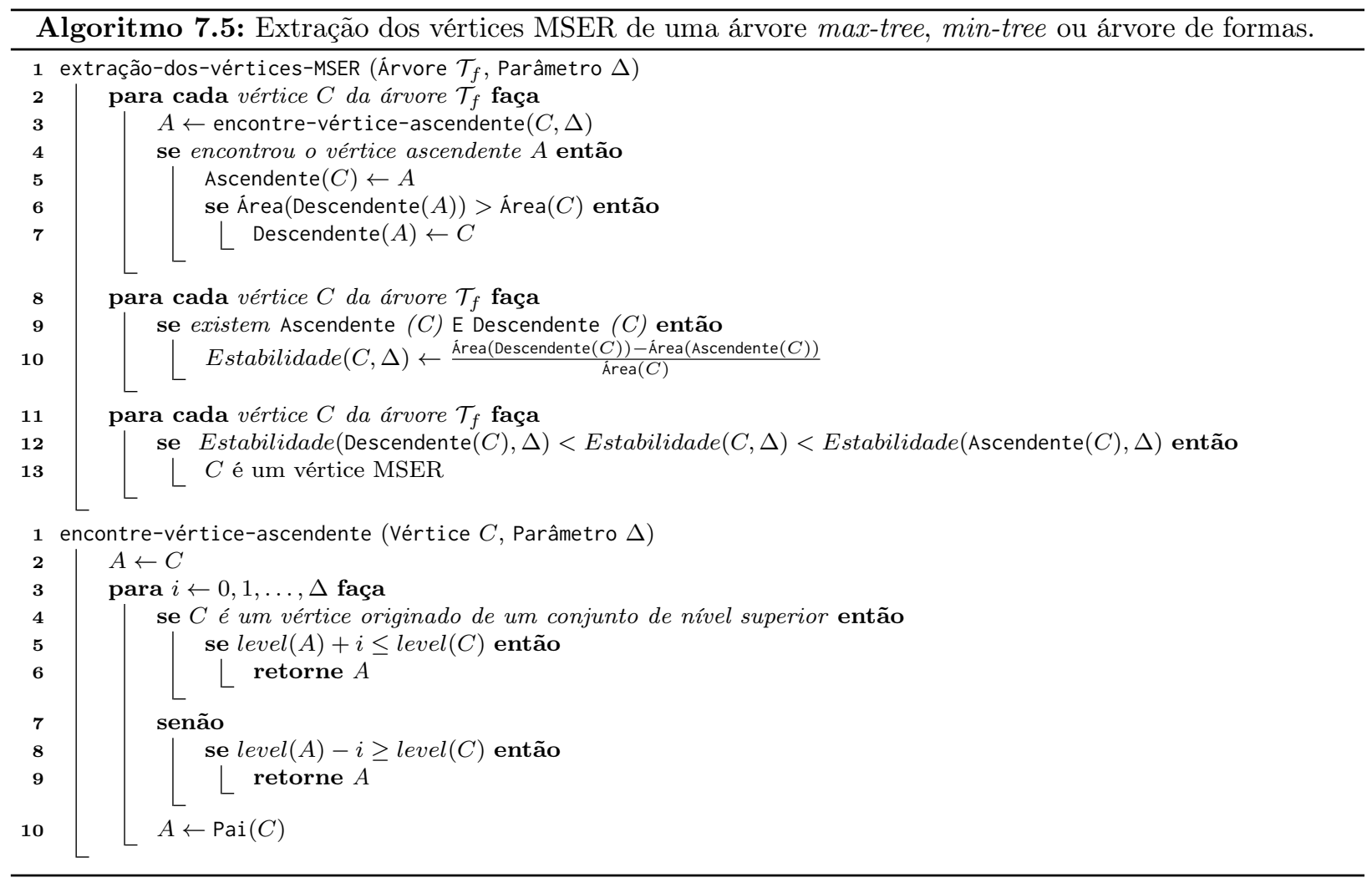

Com base nestas considerações, o classificador $\Omega_{m s e r}$ é construído para todo vértice $C \in \mathcal{P}(\mathcal{D})$ 
da seguinte forma:

$$
\Omega_{m s e r}(C)= \begin{cases}\text { não filtrar, } & \text { se } C \text { é um vértice MSER } \\ \text { filtrar, } & \text { caso contrário, }\end{cases}
$$

Diversas variantes de MSER têm sido propostas na literatura, como por exemplo, Kimmel et al. (2011) e Perd'och (2011) que definem novas funções de estabilidade que levam em consideração informações de outros atributos geométricos extraídos dos CCs. Já Xu et al. (2014) propôs uma nova abordagem para selecionar vértice baseada na teoria de Morse. Todas essas abordagens, podem ser consideradas para definir filtros de resíduos indesejáveis. Nas Figuras 7.9 e 7.10 são mostrados exemplos de aplicações dos últimos levelings por meio de filtragem dos resíduos indesejáveis baseadas em MSER. No exemplo apresentado na Figura 7.9 foi utilizada uma família de primitivas baseada
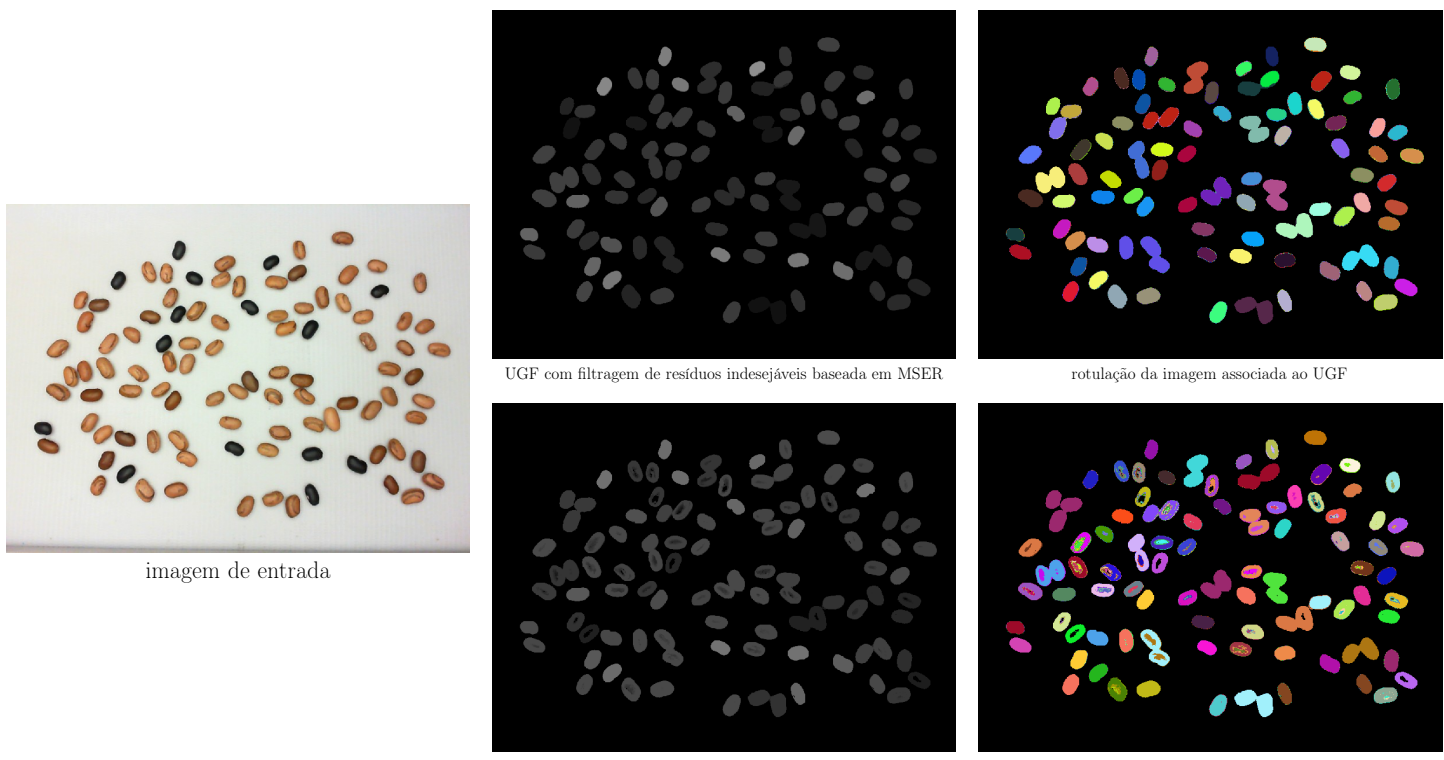

Figura 7.9: Aplicações dos últimos filtros por grãos com filtragem de resíduos indesejáveis baseadas em MSER.

em valor de extinção com atributo de área e utilizando filtragem por meio de MSER-.

No segundo exemplo apresentado na Figura 7.10 também foi utilizada uma família de primitivas baseada em valor de extinção com atributo de área e utilizando filtragem de resíduos indesejáveis com base em uma composição de filtragens: MSER seguido de filtragens baseadas em informação de formas (área, número de buracos, retangularidade, variância). 


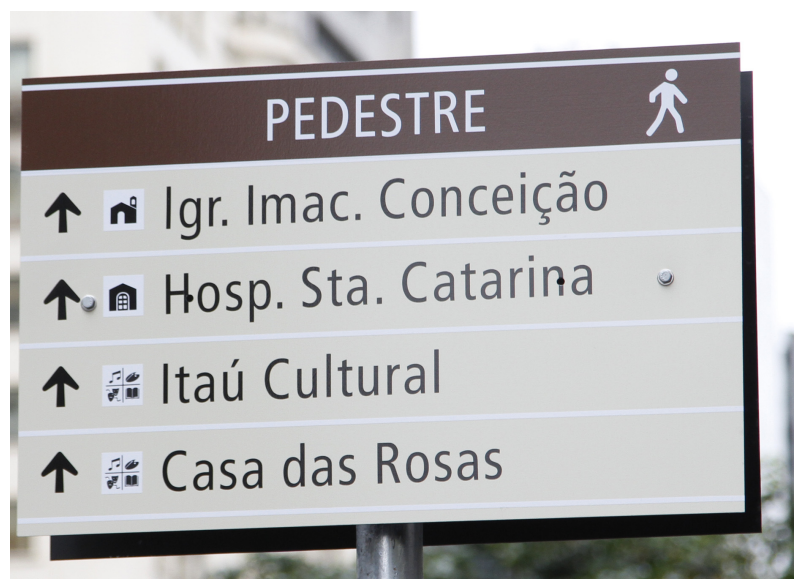

imagem de entrada (Colorida)

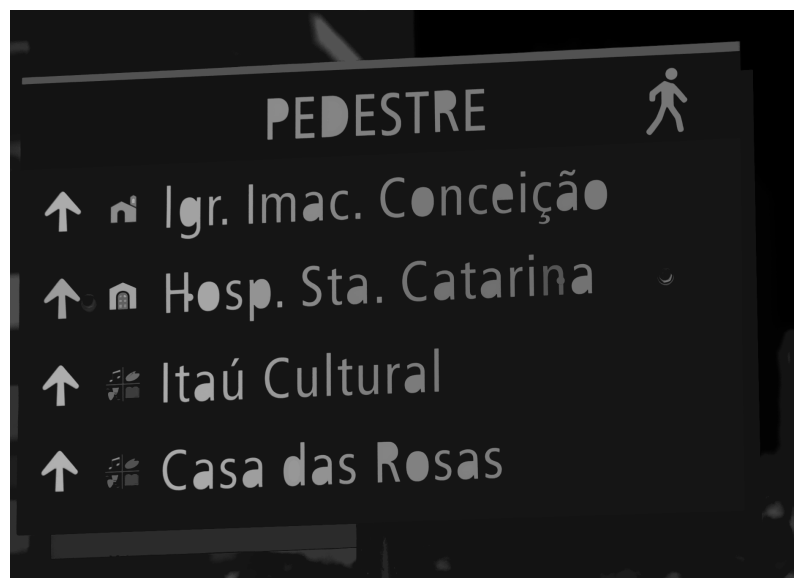

UGF com família de primitivas baseada em valores de extinção

\section{PEDESTRE}

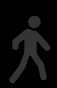

个 nil Igr. Imac. Conceição

个, n Hosp. Sta. Catarina

个 : í |taú Cultural

个 : Casa das Rosas

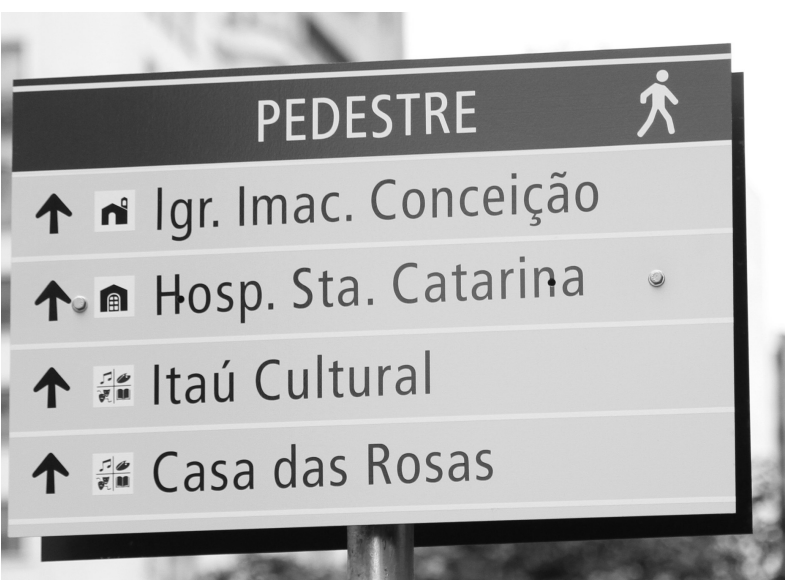

imagem de entrada (Níveis de cinca)

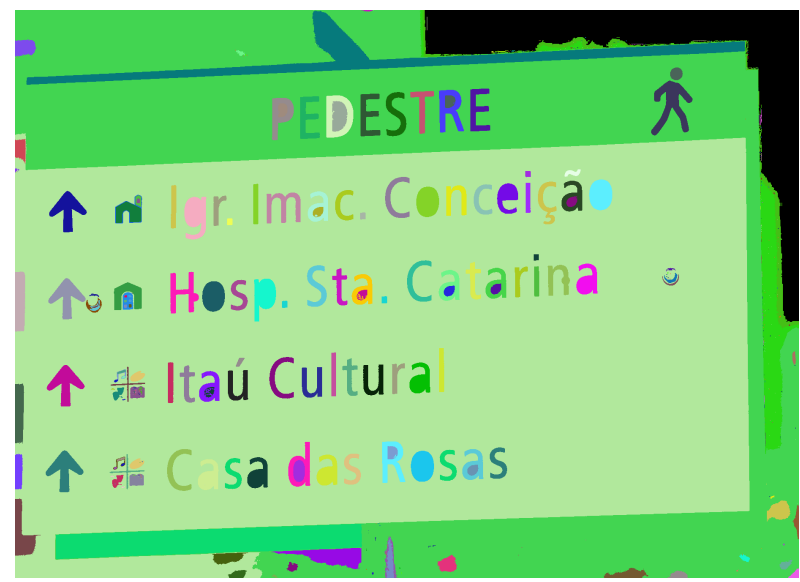

rotulação da imagem associada ao UGF

\section{PEDESTRE $\quad \dot{i}$}

$\uparrow$ ål Igr. Imac. Conceição

个, a Hosp. Sta. Catarina

个 : í |taú Cultural

$\uparrow$ iz Casa das Rosas

UGF com família de primitivas baseada em valores de extinção e composições de filtragens de resíduos indesejáveis

rotulação da imagem associada ao UGF

Figura 7.10: Aplicações dos últimos filtros por grãos com filtragem de resíduos indesejáveis baseada em uma composição de filtragens: MSER seguido de filtragens baseadas em informação de formas (área, número de buracos, retangularidade, variância). 


\section{Parte III}

\section{Aplicações}

\section{Resumo}

Esta última parte da tese é dedicada à aplicações dos últimos levelings em problemas de processamento e análise de imagens. São apresentados três problemas: (1) segmentação de vasos sanguineos em imagens de retina; (2) binarização de documentos históricos e (3) localização de texto em imagens de cenas. O primeiro problema consiste em segmentar vasos sanguineos em imagens de retinas. A abordagem proposta analisa todo o espaço de resíduos gerados por uma imagem filtrada da retina e, sobre esse espaço é projetado um filtro, com base em um descritor de alongamento, para eliminar resíduos extraídos de regiões não alongadas. Dessa forma, os pixels desse último leveling contendo resíduos não nulos, são classificados como pertencentes aos vasos sanguíneos, caso contrário, são classificados como pixels do plano de fundo. O segundo problema consiste em binarizar imagens de documentos históricos por meio dos últimos levelings. A abordagem proposta analisa o espaço de resíduos e classifica como objeto da imagem os pixels com valores residuais não nulos extraídos de regiões maximalmente estáveis. O terceiro problema consiste em localizar regiões de texto em imagens de cenas. O método proposto consiste de duas grandes etapas, sendo que na primeira etapa, é empregado o uso dos últimos levelings para extrair regiões de caracteres da imagem de referência e, em seguida, esses caracteres são agrupados formando assim um conjunto de regiões de textos candidatas. Assim, na segunda etapa, um conjunto de atributos é obtido a partir de cada região de texto candidata e é usado mais tarde como entrada para um classificador, a fim de rotular essas regiões em texto ou não-texto. Resultados experimentais utilizando imagens de bancos públicos mostram que os métodos propostos por meio dos últimos levelings são comparáveis aos outros métodos encontrados na literatura. 


\section{Segmentação de vasos sanguíneos em imagens}

\section{Resumo do capítulo}

Neste capítulo descreve-se o uso dos últimos levelings para a segmentação de vasos sanguíneos em imagens de retinas. A abordagem proposta analisa todo o espaço de resíduos gerados por uma imagem filtrada da retina e, sobre esse espaço é projetado um filtro, com base em um descritor de alongamento, para eliminar resíduos extraídos de regiões não alongadas. Dessa forma, os pixels desse último leveling contendo resíduos não nulos, são classificados como pertencentes aos vasos sanguíneos, caso contrário, são classificados como pixels do plano de fundo. Resultados experimentais utilizando imagens dos bancos públicos DRIVE e STARE mostram que a abordagem proposta, apesar de simples, é comparável aos outros métodos encontrados na literatura.

\subsection{Introdução}

A segmentação da rede de vasos sanguíneos é uma tarefa inicial e importante no processo de análise de imagens de retinas, pois por meio dela é possível identificar sinais de hipertensão, diabetes, arteriosclerose e doenças cardiovasculares (Hoover et al., 2000; Staal et al., 2004). Na Figura 8.1 é apresentada uma imagem de fundo de olho e a rede de vasos sanguíneos segmentada manualmente.

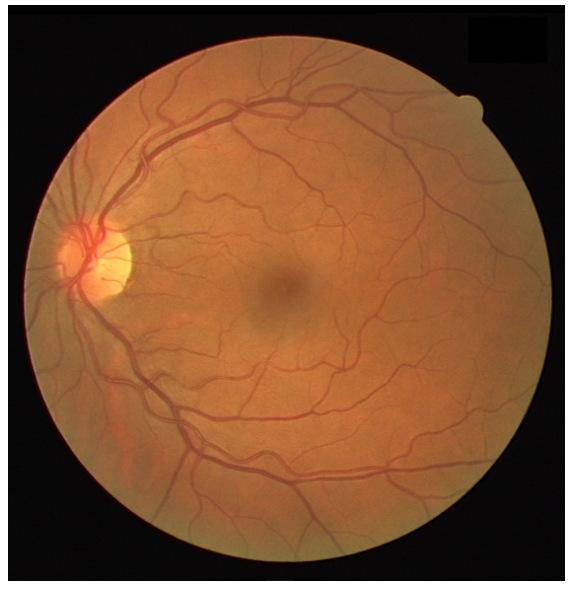

Imagem de fundo de olho

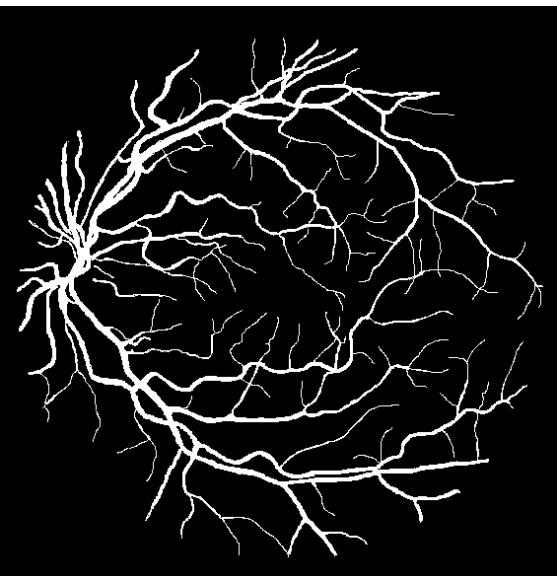

Rede de vasos sanguíneos segmentada

Figura 8.1: Exemplo de imagem de fundo de olho e sua rede de vasos sanguineos segmentada manualmente. 
Nas últimas décadas, têm surgido diversos métodos para segmentação automática de vasos sanguíneos (Al-Diri et al., 2009; Hoover et al., 2000; Jiang e Mojon, 2003; Mendonça e Campilho , 2006; Niemeijer et al., 2004; Staal et al., 2004; Xu et al., 2013). Estes métodos vêm sendo testados em bancos públicos de imagens e avaliados quantitativamente através da comparação com segmentações manuais através da análise ROC (receiver operating characteristic, ou característica de operação do receptor). Por conseguinte, é apresentado neste capítulo um método baseado nos últimos levelings para segmentar a rede de vasos sanguíneos.

\subsection{Abordagem proposta}

A grande maioria dos métodos propostos para segmentação automática de vasos sanguíneos faz uso da imagem do canal verde $f_{g} \in \mathcal{F}(\mathcal{D})$ de uma dada imagem colorida (RGB) da retina por revelar um maior contraste na rede de vasos sanguíneos (Hoover et al., 2000; Staal et al., 2004; Xu et al., 2013), como pode ser observado na Figura 8.2.

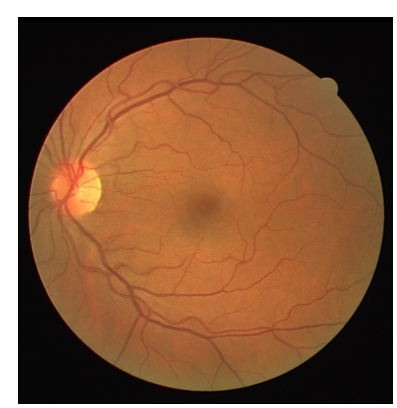

Imagem colorida (RGB)

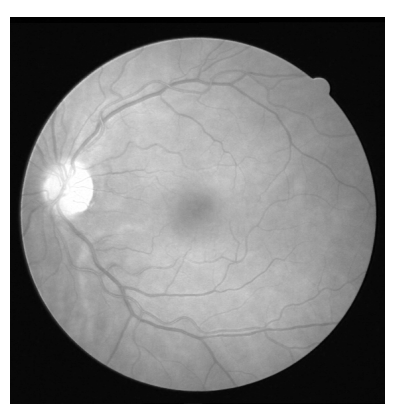

Imagem do canal vermelho

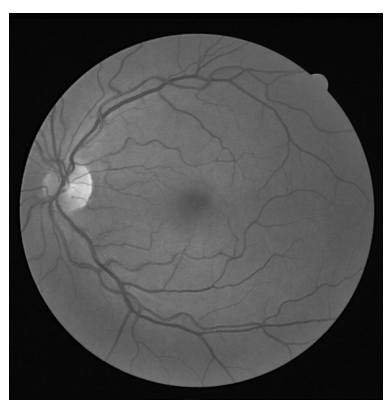

Imagem do canal verde

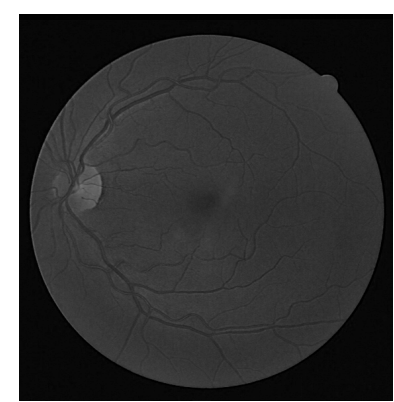

Imagem do canal azul

Figura 8.2: Exemplo de uma imagem colorida de retina.

Esta imagem $f_{g}$ contém muitas regiões contrastantes que não coincidem com regiões de vasos sanguíneos, então para eliminar estes artefatos indesejáveis da imagem $f_{g}$ e, ao mesmo tempo, preservar as regiões de vasos sanguíneos, é aplicado o operador residual fechamento top-hat $\varphi_{\mathcal{B}}^{T H}$ por um EE $\mathcal{B}$ no formato de disco cujo o raio corresponde a pelo menos a metade (mais um) da espessura máxima dos vasos, ou seja, aproximadamente 7 pixels para as imagens dos bancos públicos DRIVE (Hoover et al., 2000) e STARE (Staal et al., 2004). Dessa forma, são eliminadas as regiões de vasos sanguíneos por meio de um fechamento $\varphi_{\mathcal{B}}\left(f_{g}\right)$ e, em seguida, elas são recuperadas por uma subtração com a imagem de referência $f_{g}$, como mostrado na Figura 8.3.

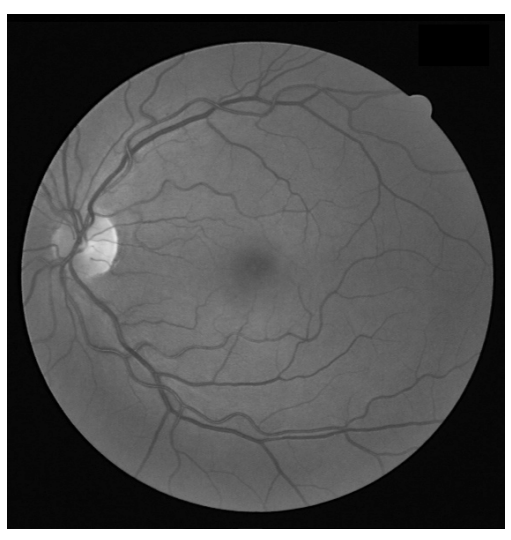

Imagem $f_{g}$

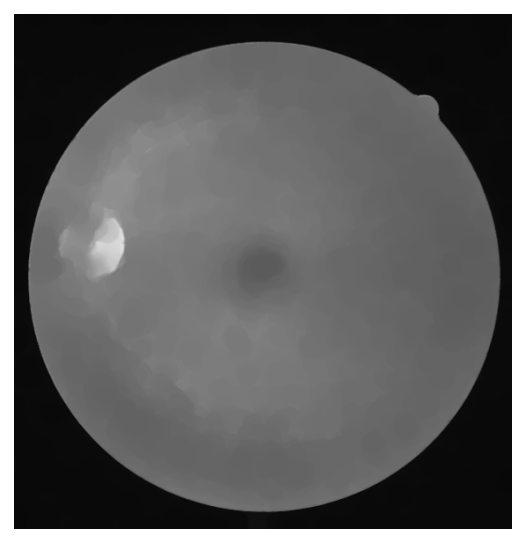

Fechamento $\varphi_{\mathcal{B}}\left(f_{g}\right)$

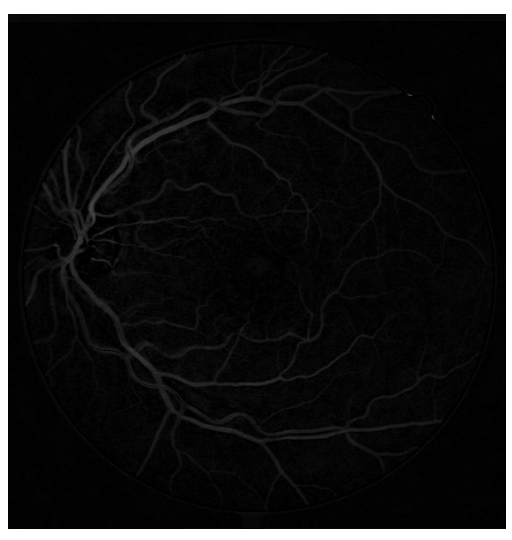

Fechamento top-hat $\varphi_{\mathcal{B}}\left(f_{g}\right)-f_{g}$

Figura 8.3: Exemplo de imagem de fundo de olho e sua rede de vasos sanguíneos. 
Nesta imagem $\varphi_{\mathcal{B}}^{T H}\left(f_{g}\right)$ aplica-se um último leveling construído com base nas seguintes observações: $(i)$ pelo fato que os objetos (vasos sanguíneos) da imagem $\varphi_{\mathcal{B}}^{T H}\left(f_{g}\right)$ são claros e seus planos de fundos serem escuros, nós usamos primitivas anti-extensivas obtidas por reconstruções superiores; (ii) como pretende-se segmentar os vasos sanguíneos é importante considerar todo o espaço de primitivas possíveis (ver Seção 7.1); (iii) assim, sobre o espaço dos resíduos $\left\{r_{i}\left(\varphi_{\mathcal{B}}^{T H}\left(f_{g}\right)\right.\right.$ ): $\left.i \in \mathcal{I}\right\}$ (ou melhor, dos conjuntos $\{\mathcal{N} r(i): i \in \mathcal{I}\})$ pode-se projetar filtros que preservem as estruturas finas e alongadas que podem estar presentes nos vértices dos conjuntos $\mathcal{N} r(i), i \in \mathcal{I}$, como por exemplo, o filtro de alongamento descrito na Seção 7.2.1. Dessa forma, sabendo que os vértices representam CCs dos conjuntos de níveis superiores podem-se produzir amostras a partir de algumas imagens de um conjunto de treinamento e assim estimar um limiar de separação dos objetos alongados dos não alongados. Esse limiar pode ser empiricamente determinado por meio da observação do valor de alongamento para algumas formas binárias, como mostrado na Figura 8.4. Note que, os vértices do conjuntos $\mathcal{N} r(i)$ contém CCs que se assemelham a estas imagens binárias apresentadas na Figura 8.4. Assim, por meio de uma análise estatística pode-se chegar a um limiar $\alpha$ e assim utilizá-lo

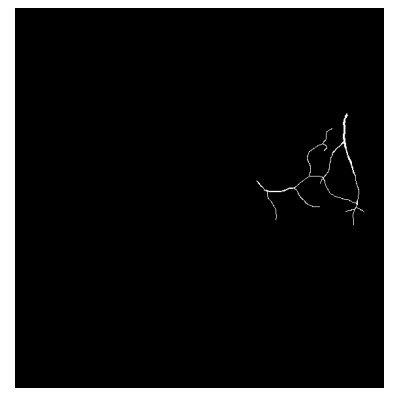

Alongamento 0,030

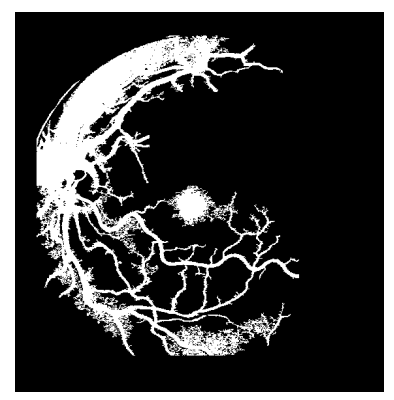

Alongamento 0,203

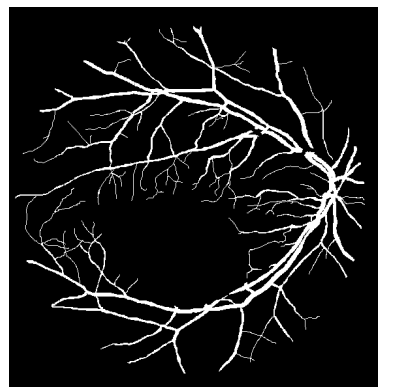

Alongamento 0, 114

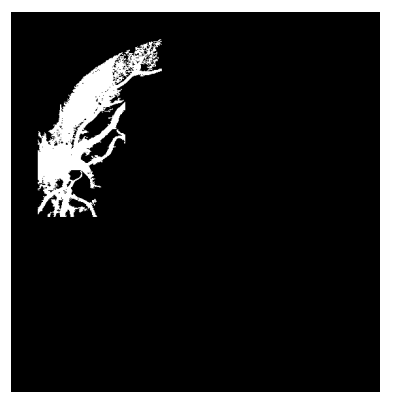

Alongamento 0, 223

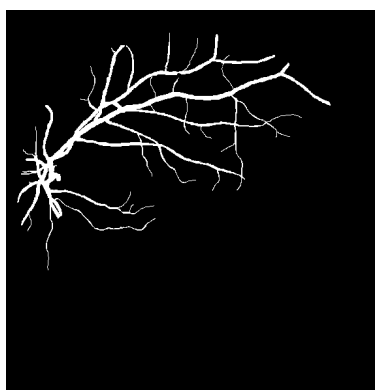

Alongamento 0,066

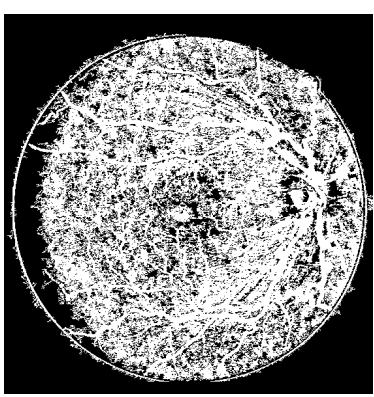

Alongamento 0,692

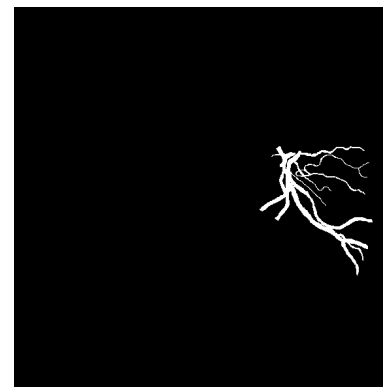

Alongamento 0, 123

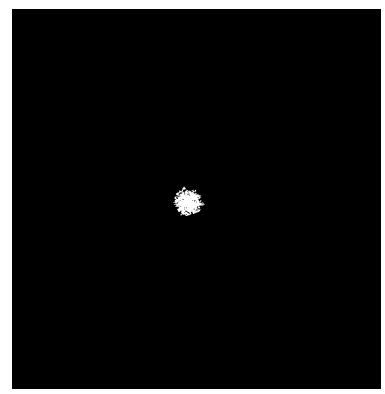

Alongamento 0, 843

Figura 8.4: Exemplo de alongamento de vasos sanguíneos segmentados de imagens de fundo de olho.

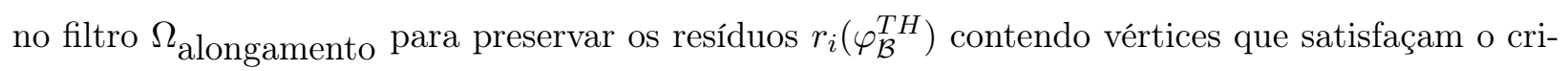
tério de alongamento. Além disso, é incluído no filtro a seguinte heurística (descartar os vértices com áreas menores ou igual à $\epsilon$ ) para diminuir a quantidade de falsos positivos. Mais precisamente, o filtro para classificar vértices com formato de estruturas alongadas é dado por

$$
\Omega_{\text {alongamento }}(C)= \begin{cases}\text { não filtrar, } & , \text { se } \frac{\text { Área }(C)}{\pi \times \text { Maior eixo }(C)^{2}} \leq \alpha \text { e Área }(C)>\epsilon, \\ \text { filtrar, } & , \text { caso contrário. }\end{cases}
$$

onde Área $(C)$ é a área da $\mathrm{CC} C$ e Maior eixo $(C)$ é o comprimento do maior eixo da elipse envolvente sobre o CC $C$ que pode ser computado incrementalmente (ver Seção 7.2.1) por meio de momentos centrais. 
Dessa forma, considere $\mathcal{R}_{\Omega}^{+}$o último leveling com filtragem $\Omega_{\text {alongamento e seja } q \text { a função }}$ associada ao último leveling $\mathcal{R}_{\Omega}^{+}$contendo os índices das primitivas que produziram os máximos resíduos não nulos. Assim, o resultado final da segmentação é determinado da seguinte forma: $\forall p \in \mathcal{D}$, se $q(p)>0$ então $p$ é um pixel que pertence a rede de vasos sanguíneos, caso contrário, $p$ pertence ao plano de fundo da imagem. Na Figura 8.5 é apresentado um exemplo da aplicação do método proposto.

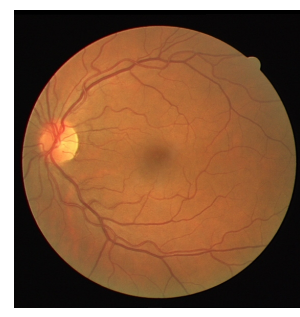

(a) Imagem colorida

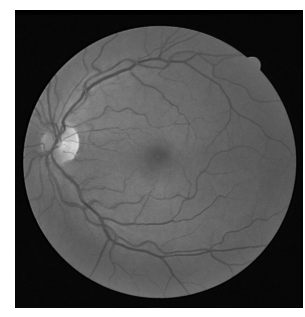

(b) Imagem do canal verde

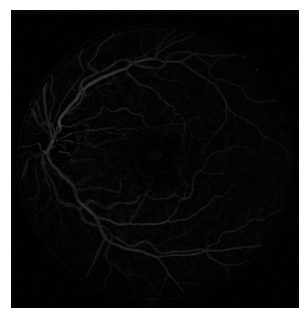

(c) Imagem do fechamento top-hat

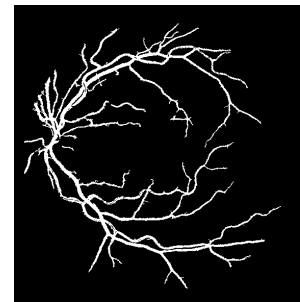

(d) Resultado da segmentação

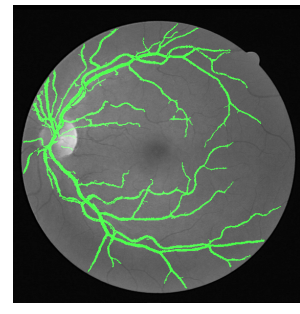

(e) Sobreposição de (b) com (d)

Figura 8.5: Ilustração mostrando os passos seguidos pelo método proposto para segmentação de vasos sanguíneos em imagens de retinas, onde (a) a partir de uma imagem de entrada $R G B$, (b) é extraída a imagem do canal verde, e em seguida, (c) é aplicado o fechamento top-hat, e depois, (d) é aplicado o último leveling com filtro de alongamento. Por fim, (e) é feito uma sobreposição da imagem de entrada com a imagem resultante para auxiliar a visualização dos vasos sanguíneos segmentados.

\subsection{Experimentos e resultados}

Para avaliar o desempenho do método proposto foram utilizadas as imagens dos bancos públicos Digital Retinal Images for Vessel Extraction (DRIVE) e Structured Analysis of the Retina (STARE). O banco DRIVE consiste de 40 imagens, sendo sete com patologia. As imagens foram escolhidas aleatoriamente de um programa de triagem para detecção de retinopatia diabética na Holanda e foram capturadas por uma câmera Canon CR5 3CCD com um campo de vista de $45^{\circ}$ e digitalizada para uma resolução de $768 \times 584$ pixels, 8 bits de profundidade por canal do modelo de cores RGB. As 40 imagens são divididas em dois conjuntos, um para treinamento e outro para teste, ambos com 20 imagens. Para cada imagem do conjunto de treinamento é fornecida uma imagem segmentada manualmente e para o conjunto de teste são fornecidas duas imagens segmentadas manualmente (Hoover et al., 2000). Já o banco STARE consiste de 20 imagens entre as quais dez apresentam patologias. Estas imagens foram capturadas por um câmera TopCon TRV-50 com um campo de vista de $35^{\circ}$ e digitalizada para um resolução $700 \times 605,8$ bits de profundidade por canal do modelo de cores RGB. Também são fornecidas duas imagens segmentadas manualmente para cada imagem do banco (Staal et al., 2004).

Para avaliação do método proposto são empregadas as medidas de desempenho sensibilidade (também chamada de taxa de acerto), especificidade e acurácia (Staal et al., 2004). A sensibilidade mensura a taxa de verdadeiros positivos, a especificidade mensura a taxa de verdadeiros negativos e a acurácia mensura a taxa dos pixels classificados corretamente. Mais precisamente,

$$
\begin{aligned}
& \text { sensibilidade }=\frac{V P}{V P+F N}, \\
& \text { especificidade }=\frac{V N}{V N+F P},
\end{aligned}
$$




$$
\text { acurácia }=\frac{V P+V N}{V P+V N+F P+F N},
$$

onde $V P$ e $V N$ são as quantidades de verdadeiros positivos e negativos, respectivamente e $F P$ e $F N$ são as quantidades de falsos positivos e negativos, respectivamente.

Nas Figuras 8.6 e 8.7 são apresentadas quatro resultados de segmentações de vasos sanguíneos em imagens de retinas (com e sem patologias) extraídas dos bancos DRIVE e STARE, respectivamente. Percebe-se que a maioria dos vasos sanguíneos foram extraídos corretamente, embora haja presenças de pequenos ruídos e os vasos sanguíneos extremamente finos são perdidos. Vale salientar, que todas as imagens foram segmentadas utilizando os mesmos parâmetros.
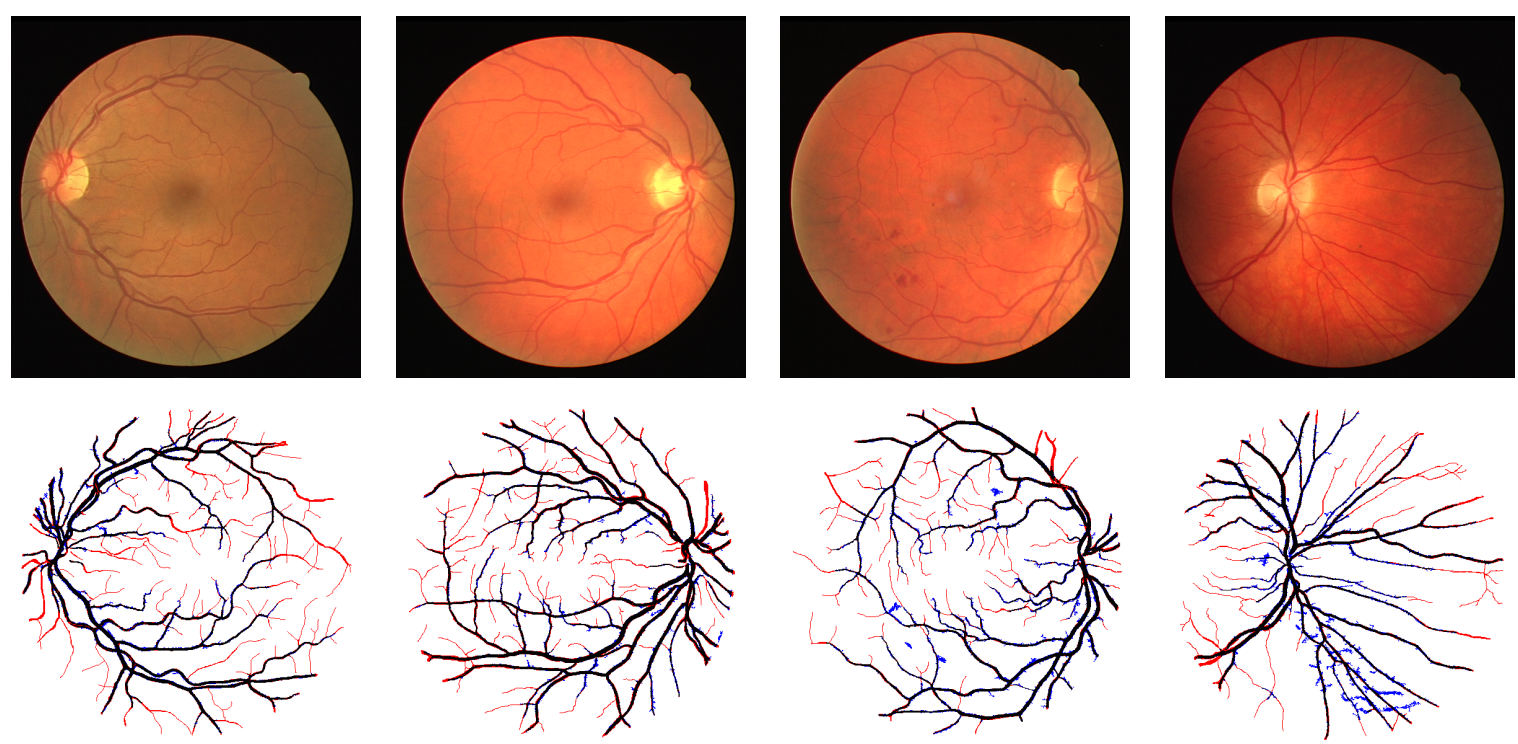

Figura 8.6: Resultado de quatro segmentações de vasos sanguíneos das 20 imagens testadas do banco DRIVE. No topo estão as imagens coloridas de entrada e em baixo as segmentações correspondentes. Note que, os pixels em preto são verdadeiros positivos, em branco os verdadeiros negativos, em azul os falsos positivos, e em vermelho os falsos negativos.
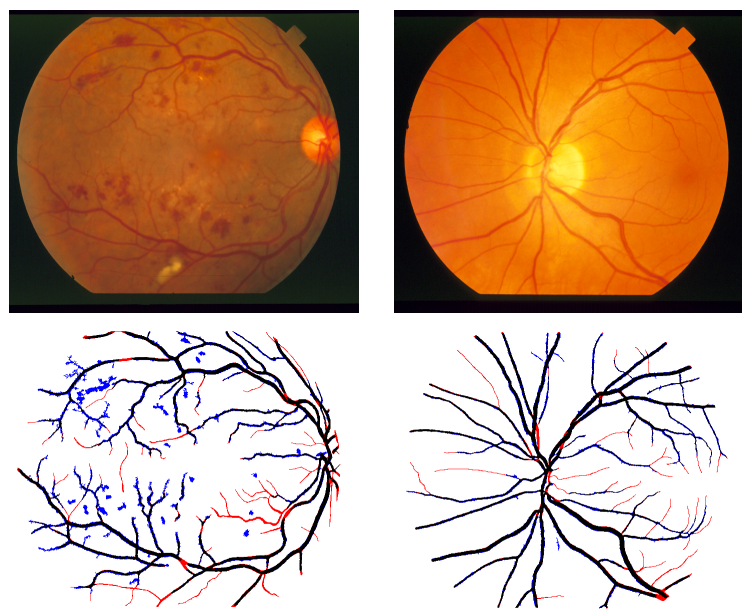
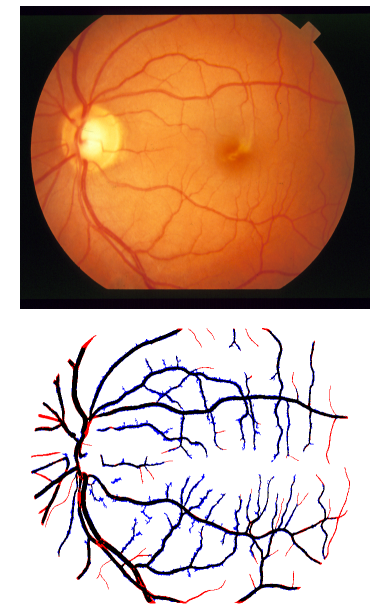
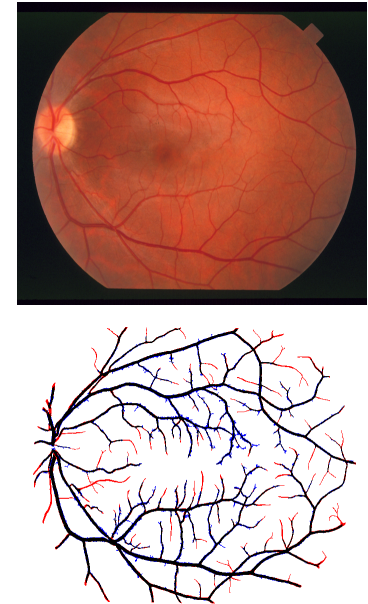

Figura 8.7: Resultado de quatro segmentações de vasos sanguíneos das 20 imagens testadas do banco STARE. No topo estão as imagens de entrada coloridas e em baixo as segmentações correspondentes. Note que, os pixels em preto são verdadeiros positivos, em branco os verdadeiros negativos, em azul os falsos positivos, e em vermelho os falsos negativos. 
Resultados comparativos de diferentes abordagens, incluindo o método proposto, são apresentadas nas Tabelas 8.1 e 8.2. Pode-se perceber, analisando os resultados que o método proposto é comparável a outras abordagens apesar de usarmos um único atributo para descrever os vasos sanguíneos. Contudo, é importante ressaltar que, se consideramos um conjunto de atributos extraídos dos vértices presentes em $\mathcal{N} r(i)$, pode-se construir classificadores cujo os resultados tendem a ser bem melhores.

Tabela 8.1: Tabela comparativa de diferentes métodos para segmentação de vasos sanguineos nas imagens do banco DRIVE.

\begin{tabular}{|c|c|c|c|}
\hline Método & Sensibilidade & Especificidade & Acurácia \\
\hline Segundo especialista & 0,7761 & 0,9725 & 0,9473 \\
\hline Mendonça e Campilho (2006) & 0,7344 & 0,9764 & 0,9452 \\
\hline Staal et al. (2004) & 0,7193 & 0,9773 & 0,9441 \\
\hline Método proposto & 0,6902 & 0,9800 & 0,9429 \\
\hline Niemeijer et al. (2004) & 0,6793 & 0,9801 & 0,9416 \\
\hline Xu et al. (2013) & 0,6924 & 0,9779 & 0,9413 \\
\hline Al-Diri et al. (2009) & - & - & 0,9258 \\
\hline Jiang e Mojon (2003) & 0,6478 & 0,9625 & 0,9222 \\
\hline
\end{tabular}

Tabela 8.2: Tabela comparativa de diferentes métodos para segmentação de vasos sanguíneos nas imagens do banco STARE.

\begin{tabular}{|c|c|c|c|}
\hline Método & Sensibilidade & Especificidade & Acurácia \\
\hline Segundo especialista & 0,8949 & 0,9390 & 0,9354 \\
\hline Jiang e Mojon (2003) & - & - & 0,9513 \\
\hline Xu et al. (2013) & 0,7149 & 0,9749 & 0,9471 \\
\hline Método proposto & 0,6835 & 0,9739 & 0,9460 \\
\hline Mendonça e Campilho (2006) & 0,6996 & 0,9730 & 0,9440 \\
\hline Hoover et al. (2000) & 0,6751 & 0,9567 & 0,9267 \\
\hline
\end{tabular}

\subsection{Conclusão}

Neste capítulo apresentou-se a aplicação dos últimos levelings em um problema de segmentação de vasos sanguíneos em imagens de retinas. O método proposto, apesar de usar um único atributo, seus resultados são comparáveis a outras abordagens. O tempo médio para processar uma imagem dos bancos STARE ou DRIVE é em torno de 0,95 segundos em um computador com processador i7 e $8 G B$ de memória RAM. Futuramente, pretende-se melhorar a acurácia do método proposto por meio do uso de um classificador mais robusto. 


\title{
Binarização de texto em imagens de
} documentos históricos

\begin{abstract}
Resumo do capítulo
Neste capítulo é apresentada uma aplicação para binarização de documentos históricos por meio dos últimos levelings. A abordagem proposta analisa todo o espaço de resíduos e classifica como objeto da imagem os pixels com valores residuais não nulos extraídos de regiões maximalmente estáveis. Resultados experimentais utilizando os bancos de imagens dos concursos DIBCOs evidenciam que a abordagem proposta apresenta resultados semelhantes aos métodos vencedores do concursos DIBCOs.
\end{abstract}

\subsection{Introdução}

Nos últimos anos tem surgido a necessidade de armazenar e indexar imagens de documentos históricos produzidos há muitas décadas ou mesmo séculos. Estes documentos costumam possuir imperfeições como manchas, dobras, sinais de envelhecimento, entre outras. Consequentemente, softwares de reconhecimento ótico de caracteres (OCR - do inglês, Optical Character Recognition) acabam tendo desempenho insatisfatório devido à essas imperfeições, como pode ser percebido nas imagens ilustradas na Figura 9.1.
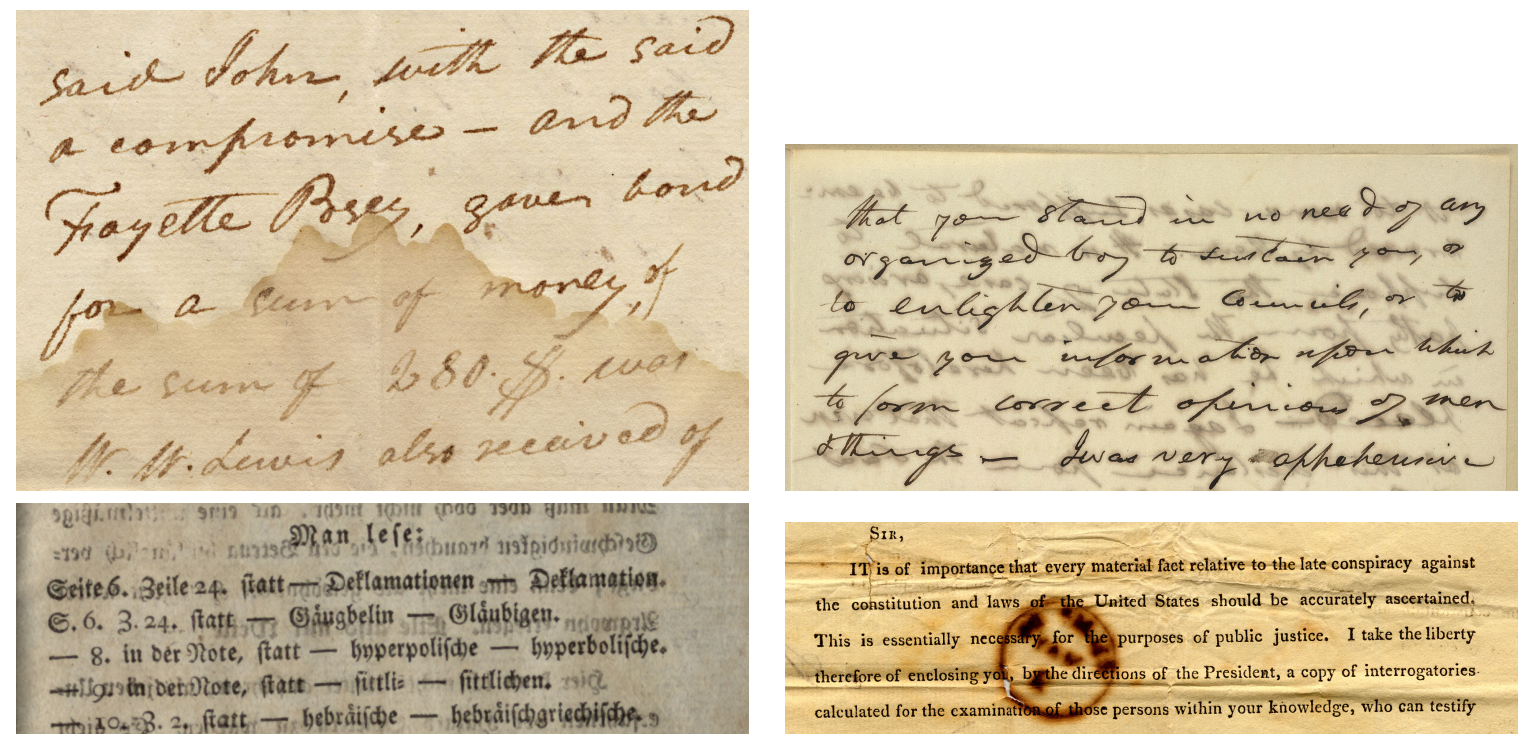

Sik,

IT is of importance that every material fact relative to the late conspiracy against the constitution and laws of the United States should be accurately ascertained. This is essentially necessany for the purposes of public justice. I take the liberty therefore of enclosing you, bycthe directions of the President, a copy of interrogatories calculated for the cxamination of those persons within your knowledge, who can testify

Figura 9.1: Exemplos de imagens de documentos históricos extraídas dos bancos dos concursos DIBCOs. 
Neste cenário, a binarização de textos em imagens de documentos históricos tem ganhado cada vez mais a atenção, dando origem, inclusive, a concursos para binarização dessas imagens (Gatos et al. , 2009; Pratikakis et al., 2013, 2010, 2011, 2012), uma vez que seu resultado é crucial para etapas posteriores do processo de reconhecimento de textos. Dadas essas considerações, é apresentado neste capítulo um método para binarização de textos em imagens de documentos históricos por meio dos últimos leveligns.

\subsection{Abordagem proposta}

O operador de binarização proposto é projetado levando em consideração as características das imagens dos concursos DIBCOs (do inglês - Document Image Binarization Contest), as quais consistem em digitalizações de documentos impressos e escritos à mão, sendo que os textos dos documentos são escuros e o seu plano de fundo é mais claro em relação ao texto. Também, os documentos digitalizados contém uma série de imperfeições, como manchas, amassados, dobras, entre outras. Neste sentido, é importante simplificar uma imagem o máximo possível, preservando ao mesmo tempo os textos nela contidos, antes de segmentá-la. Para este fim, é aplicado um fechamento top-hat $\varphi_{\mathcal{B}}^{T H}$ por um EE $\mathcal{B}$ no formato de disco cujo o raio corresponde a pelo menos a metade (mais um) da espessura máxima dos caracteres, ou seja, aproximadamente 9 pixels para imagens de documentos escritos à mão e 21 para imagens de documentos impressos. Na Figura 9.2 são apresentados dois exemplos de fechamentos top-hats em imagens dos concursos DIBCOs.

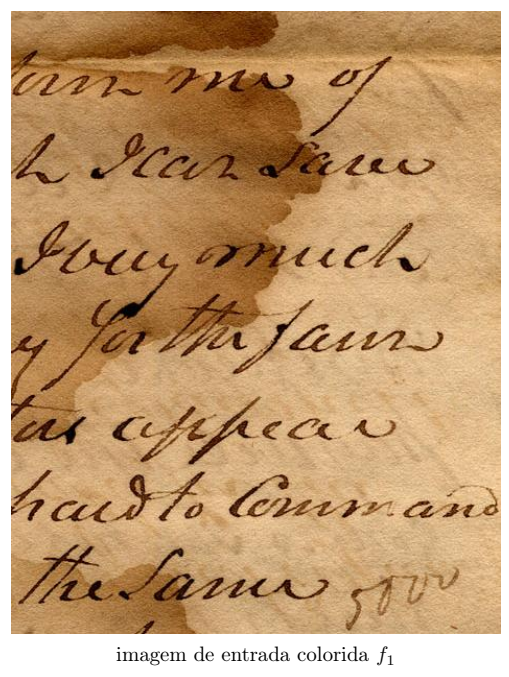

Pythagorica. D, I4.

BCB beocate. E. $40,21 \cdot 33$.

Pectniting. E. 50. 24.40. miegula. E. 66 . A.52. D.7\% oenter Rieget. E. 55.21 .42 . rechinung. E. 46.21 .38 .

¿ Regel. E. 56.21 .44 . D. 5 .

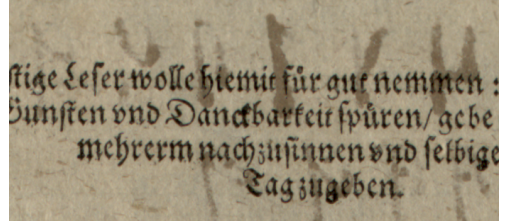

imagem de entrada colorida $f_{2}$

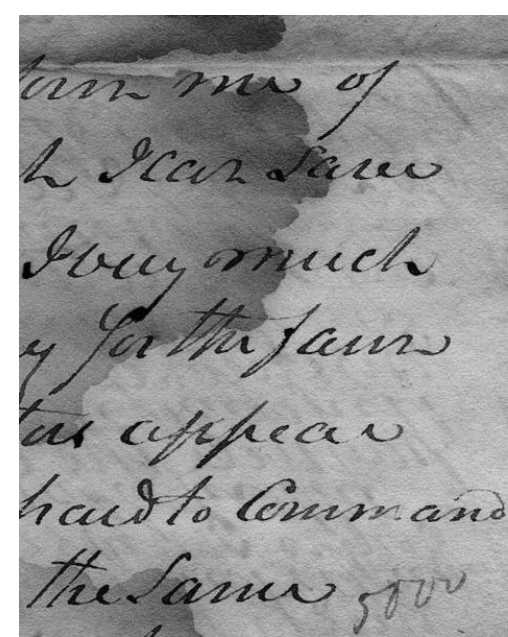

imagem de entrada $f_{1}$ em níveis de cinza

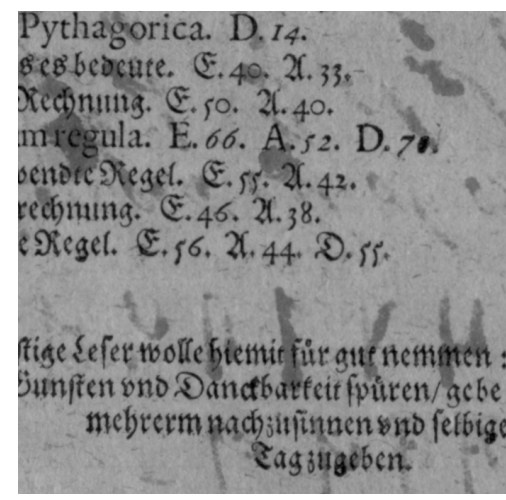

imagem de entrada $f_{2}$ em níveis de cinza

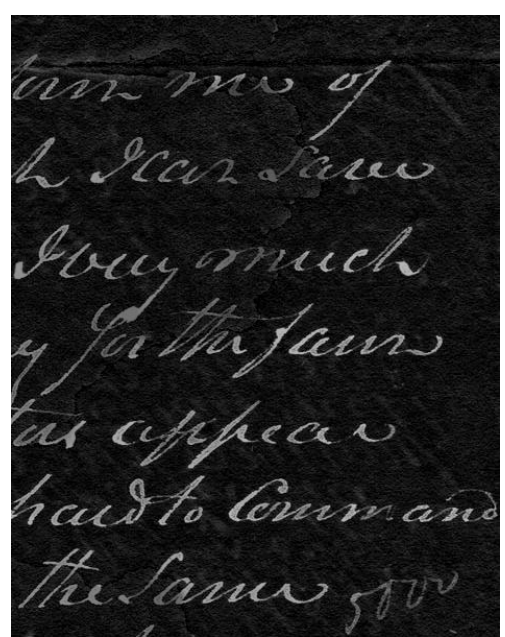

fechamento top-hat $\varphi_{B}^{T H}\left(f_{1}\right)$

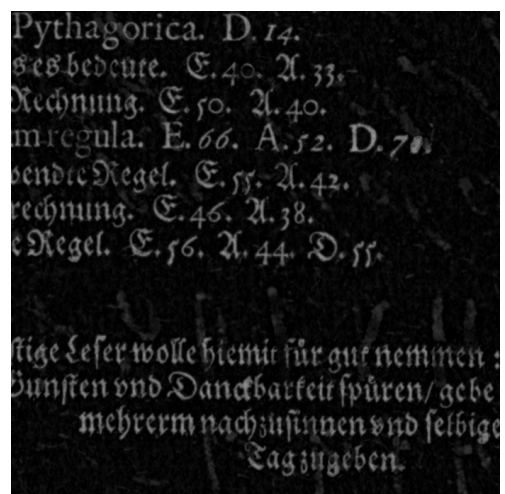

fechamento top-hat $\varphi_{\mathcal{B}}^{T H}\left(f_{2}\right)$

Figura 9.2: Exemplos de aplicações de fechamentos top-hats em imagens de documentos históricos.

Nesta imagem $\varphi_{\mathcal{B}}^{T H}(f)$ aplica-se um último leveling construído com base nas seguintes obser- 
vações: $(i)$ como os objetos (textos) da imagem $\varphi_{\mathcal{B}}^{T H}(f)$ são claros e seus planos de fundos serem escuros, nós usamos primitivas anti-extensivas obtidas por reconstruções superiores; (ii) como pretende-se segmentar os caracteres, é importante considerar todo o espaço de primitivas possíveis; (iii) como as regiões dos caracteres estão compostas por máximas regionais e existem nessas imagens dos concursos DIBCOs pelo menos 30 níveis de contraste entre os caracteres do seu plano de fundo, então é esperado que regiões dos caracteres com uma diferença em torno de 10 a 15 níveis sejam maximalmente estáveis, como pode-se perceber na Figura 9.3.

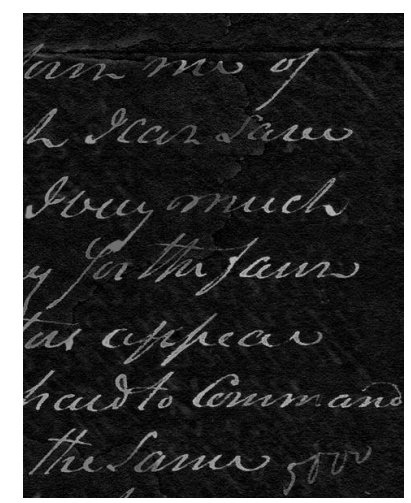

fechamento top-hat $\varphi_{\mathcal{B}}^{T H}\left(f_{1}\right)$

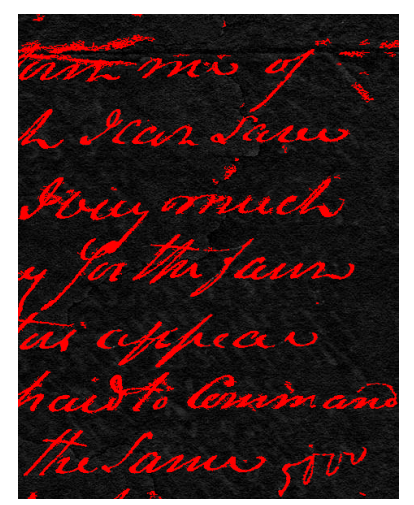

em vermelho as regiões MSER+ presentes em $\varphi_{B}^{T H}\left(f_{1}\right)$

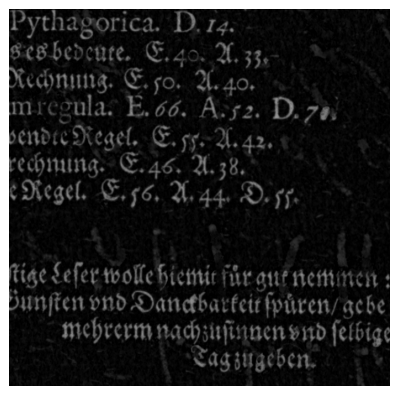

fechamento top-hat $\varphi_{\mathcal{B}}^{T H}\left(f_{2}\right)$

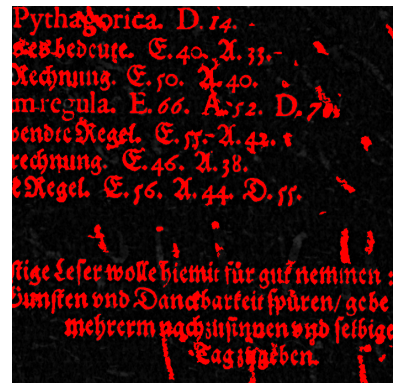

em vermelho as regiôes MSER+ presentes em $\varphi_{B}^{T H}\left(f_{2}\right)$

Figura 9.3: Exemplos de imagens de documentos históricos dos bancos dos concursos DIBCOs destacando em vermelho as regiões maximalmente estáveis.

Assim, sobre o espaço dos resíduos $\left\{r_{i}\left(\varphi_{\mathcal{B}}^{T H}(f)\right): i \in \mathcal{I}\right\}$ (ou melhor, dos conjuntos $\{\mathcal{N} r(i)$ : $i \in \mathcal{I}\}$ ) podem ser projetados filtros que preservem vértices maximamente estáveis que podem estar presentes nos conjuntos $\mathcal{N} r(i), i \in \mathcal{I}$. Além disso, são incluídos no filtro heurísticas relacionadas à geometria dos caracteres a fim de filtrar regiões que não correspondem aos caracteres. Mais precisamente:

$$
\Omega_{\text {binarização }}(C)= \begin{cases}\text { não filtrar, } & \text { se } C \text { é MSER e } H_{1}(C) \text { e } H_{2}(C) \text { são verdadeiros, } \\ \text { filtrar, } & \text { caso contrário, }\end{cases}
$$

onde $H_{1}$ e $H_{2}$ são tradicionais heurísticas empregadas em problemas de localização de texto, isto é,

$$
H_{1}(C)=\frac{\max \{\operatorname{Largura}(C), \operatorname{Altura}(C)\}}{\min \{\operatorname{Largura}(C), \operatorname{Altura}(C)\}} \leq 8 \text { e } H_{2}(C)=0,2 \leq \frac{\text { Área }(C)}{\operatorname{Largura}(C) \times \operatorname{Altura}(C)} \leq 0,8
$$

tal que a Área $(C)$, a Largura $(C)$ e a $\operatorname{Altura}(C)$ são respectivamente, a área, a largura e a altura do retângulo envolvente sobre o CC $C$.

Dessa forma, considere $\mathcal{R}_{\Omega}^{+}$o último leveling com filtragem $\Omega_{\text {binarização, e seja } q \text { a função }}$ associada ao último leveling $\mathcal{R}_{\Omega}^{+}$que contém os índices das primitivas que produziram os máximos resíduos não nulos. Assim, o resultado final da binarização é determinado da seguinte forma: $\forall p \in \mathcal{D}$, se $q(p)>0$ então $p$ é um pixel que pertence ao objeto, caso contrário, $p$ pertence ao plano de fundo da imagem.

\subsection{Experimentos e resultados}

Para avaliar o desempenho do método proposto foram utilizadas as imagens dos concursos DIBCOs dos anos entre 2009 a 2013 (Gatos et al., 2009; Pratikakis et al., 2013, 2010, 2011, 2012). O banco DIBCO-2009 consiste de 10 imagens, sendo 5 de documentos impressos e 5 de documentos 
escritos à mão; o banco DIBCO-2010 é composto por 10 imagens de documentos escritos à mão; o banco DIBCO-2011 consiste de 16 imagens, sendo 8 de documentos impressos e 8 de documentos escritos à mão; o banco DIBCO-2012 contém 14 imagens de documentos escritos à mão; o banco DIBCO-2013 consiste de 16 imagens, sendo 8 de documentos impressos e 8 de documentos escritos à mão. Todos os bancos de imagens DIBCOs fornecem, para cada imagem de documento, uma imagem segmentada manualmente para avaliar o resultado da segmentação. Para avaliação, foram empregadas as medidas de desempenho utilizadas pelos concursos DIBCOs, isto é, sensibilidade (ver Equação 8.1), especificidade (ver Equação 8.2), além de F-Measure e PSNR (Gatos et al. , 2009; Pratikakis et al., 2013, 2010, 2011, 2012), definidas a seguir.

$$
\begin{gathered}
\text { F-measure }=\frac{2 \times \text { sensibilidade } \times \text { especificidade }}{\text { sensibilidade } \times \text { especificidade }} \\
\mathrm{PSNR}=10 \times \log \left(\frac{D^{2}}{\mathrm{MSE}}\right)=10 \times \log \left(\frac{F P+F N+V P+V N}{F P+F N}\right),
\end{gathered}
$$

onde a medida F-measure é uma média ponderada das medidas sensibilidade e especificidade, PSNR é uma medida de proximidade entre o resultado e a saída esperada, ou seja, a razão entre os sinais de picos e os ruídos. O PSNR (que pode ser simplificado para dados binários) utiliza o cálculo do erro quadrático médio (MSE, do inglês - mean square error) e $D^{2}$ que é a diferença ao quadrado entre o resultado e a saída esperada. Na Figura 9.4 são apresentados quatro resultados de binarizações de imagens de documentos históricos.
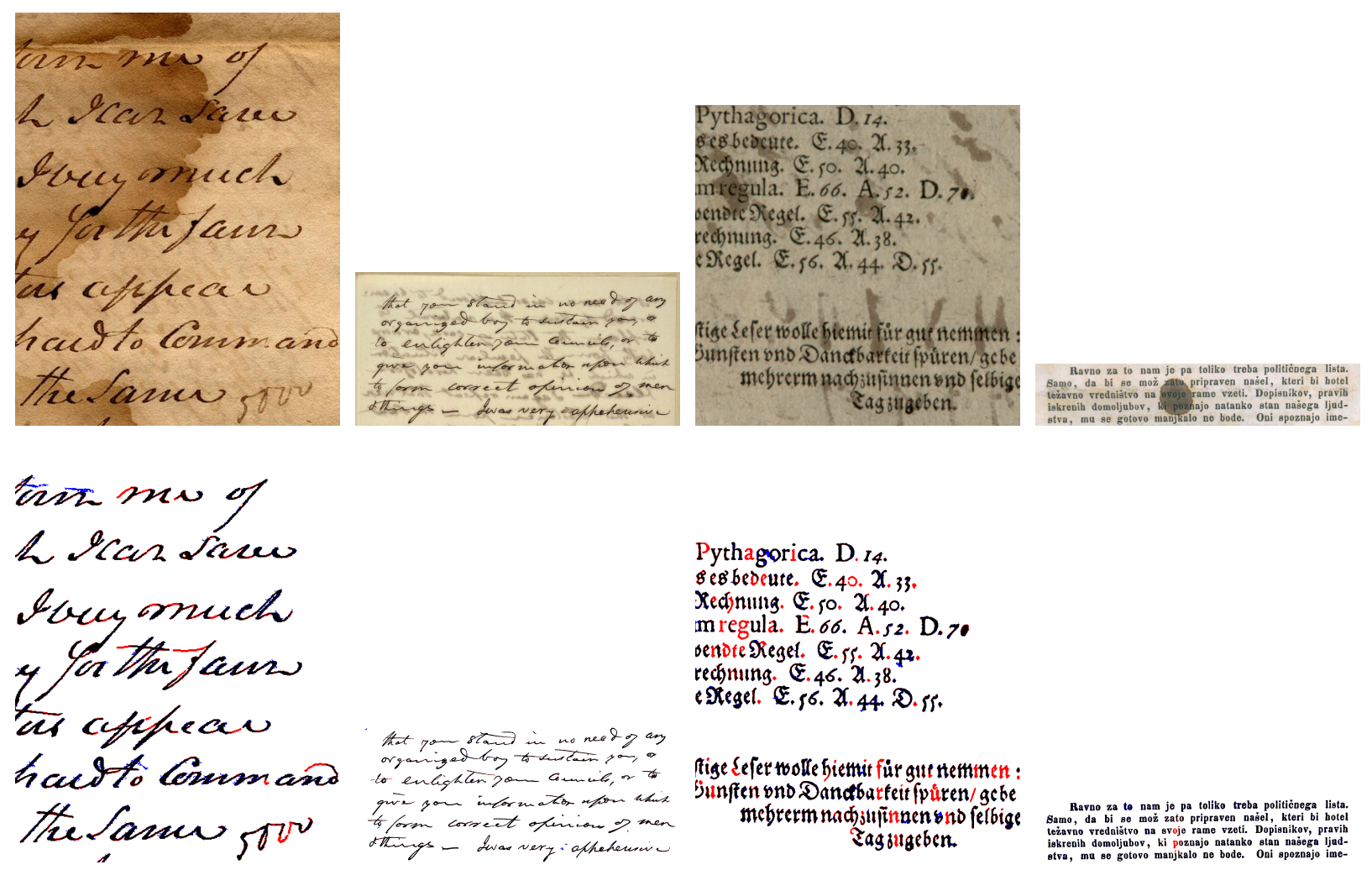

Figura 9.4: Resultados de quatro binarizações de imagens de documentos históricos. No topo estão as imagens de entrada coloridas e abaixo delas as segmentações correspondentes. Note que os pixels em preto são verdadeiros positivos, em branco os verdadeiros negativos, em azul os falsos positivos, e em vermelho os falsos negativos.

Os resultados de diferentes abordagens, incluindo o método proposto, são apresentados nas 
Tabelas 9.1 e 9.2 .

Tabela 9.1: Tabela comparativa com resultados obtidos com as imagens de documentos impressos.

\begin{tabular}{|c|c|c|c|c|}
\hline Método & F-measure & Sensibilidade & Especificidade & PSNR \\
\hline \hline \multicolumn{5}{|c|}{ Banco de imagens do concurso DIBCO-2009 } \\
\hline Método proposto & 0,9180 & 0,9540 & 0,8857 & 16,68 \\
\hline Método vencedor do DIBCO-2009 & 0,9124 & - & - & 18,66 \\
\hline Otsu (1979) & 0,9120 & 0,9347 & 0,8927 & 16,67 \\
\hline Su et al. (2010) & 0,8905 & 0,8355 & 0,9602 & 15,75 \\
\hline White e Rohrer (1983) & 0,8100 & 0,7135 & 0,9491 & 14,67 \\
\hline Sauvola e Pietikäinen (2000) & 0,6220 & 0,4694 & 0,9959 & 11,26 \\
\hline Niblack (1985) & 0,5125 & 0,8569 & 0,3705 & 6,26 \\
\hline \hline \multicolumn{4}{|c|}{ Banco de imagens do concurso DIBCO-2011 } \\
\hline Método proposto & 0,8635 & 0,9028 & 0,8438 & 15,99 \\
\hline Su et al. (2010) & 0,8121 & 0,8410 & 0,8462 & 15,04 \\
\hline Método vencedor do DIBCO-2011 & 0,8086 & - & - & - \\
\hline White e Rohrer (1983) & 0,7799 & 0,7362 & 0,8624 & 14,06 \\
\hline Otsu (1979) & 0,7776 & 0,9095 & 0,7627 & 14,15 \\
\hline Sauvola e Pietikäinen (2000) & 0,6073 & 0,4642 & 0,9973 & 12,96 \\
\hline Niblack (1985) & 0,4154 & 0,8567 & 0,2923 & 5,78 \\
\hline \hline \multicolumn{5}{|c|}{ Banco de imagens do concurso DIBCO-2013 } \\
\hline Método vencedor do DIBCO-2013 & 0,9212 & - & - & 20,68 \\
\hline Su et al. (2010) & 0,8962 & 0,9074 & 0,8936 & 17,21 \\
\hline Método proposto & 0,8906 & 0,9359 & 0,8638 & 17,02 \\
\hline Otsu (1979) & 0,8145 & 0,9167 & 0,7730 & 14,60 \\
\hline White e Rohrer (1983) & 0,8115 & 0,7380 & 0,9216 & 14,59 \\
\hline Sauvola e Pietikäinen (2000) & 0,5670 & 0,4392 & 0,9871 & 12,57 \\
\hline Niblack (1985) & 0,4262 & 0,8752 & 0,2974 & 5,93 \\
\hline
\end{tabular}

\subsection{Conclusão}

Neste capítulo foi apresentada uma aplicação dos últimos levelings em um problema de binarização de documentos históricos. Resultados experimentais utilizando as imagens e métricas dos concursos DIBCOs evidenciam que a abordagem proposta tem bons resultados para binarização de documentos históricos, principalmente em documentos impressos. O tempo médio para o processamento de uma imagem ficou em torno de 1, 4 segundos em um computador com processador $i 7$ e $8 G B$ de memória RAM. Vale lembrar que as imagens têm resoluções bem variadas, estando aproximadamente entre $469 \times 597$ a $3297 \times 1097$ pixels. Futuramente, pretende-se combinar a abordagem proposta com métodos de binarizações locais, onde os pixels presentes nos vértices contendo resíduos não nulos podem ser classificados por uma outra técnica de binarização local. Assim, pode-se parametrizar o método para aumentar a sensibilidade e, por meio de uma binarização local, eliminar os pixels classificados erroneamente. 
Tabela 9.2: Tabela comparativa com resultados obtidos com as imagens de documentos escritos à mão.

\begin{tabular}{|c|c|c|c|c|}
\hline Método & F-measure & Sensibilidade & Especificidade & PSNR \\
\hline \multicolumn{5}{|c|}{ Banco de imagens do concurso DIBCO-2009 } \\
\hline Método vencedor do DIBCO-2009 & 0,9124 & - & - & 18,66 \\
\hline Su et al. (2010) & 0,8789 & 0,8263 & 0,9496 & 19,61 \\
\hline Método proposto & 0,8048 & 0,9304 & 0,7385 & 16,94 \\
\hline Otsu (1979) & 0,6760 & 0,8895 & 0,6454 & 14,50 \\
\hline White e Rohrer (1983) & 0,4927 & 0,3723 & 0,9733 & 14,95 \\
\hline Sauvola e Pietikäinen (2000) & 0,2375 & 0,1849 & 0,9819 & 14,08 \\
\hline Niblack (1985) & 0,2544 & 0,8609 & 0,1548 & 5,29 \\
\hline \multicolumn{5}{|c|}{ Banco de imagens do concurso DIBCO-2010 } \\
\hline Método vencedor do DIBCO-2010 & 0,9150 & - & - & 19,78 \\
\hline Método proposto & 0,8459 & 0,8841 & 0,8377 & 17,23 \\
\hline Otsu (1979) & 0,8379 & 0,7650 & 0,9372 & 11,96 \\
\hline Su et al. (2010) & 0,8233 & 0,7696 & 0,9268 & 17,33 \\
\hline White e Rohrer (1983) & 0,4803 & 0,3667 & 0,9902 & 14,08 \\
\hline Sauvola e Pietikäinen (2000) & - & - & - & - \\
\hline Niblack (1985) & 0,2845 & 0,8574 & 0,1726 & 5,35 \\
\hline \multicolumn{5}{|c|}{ Banco de imagens do concurso DIBCO-2011 } \\
\hline Método proposto & 0,8707 & 0,8544 & 0,8956 & 17,69 \\
\hline Su et al. (2010) & 0,8499 & 0,8483 & 0,8614 & 17,08 \\
\hline Método vencedor do DIBCO-2011 & 0,8086 & - & - & - \\
\hline Otsu (1979) & 0,7682 & 0,8522 & 0,7452 & 15,07 \\
\hline White e Rohrer (1983) & 0,7944 & 0,7621 & 0,8590 & 15,80 \\
\hline Sauvola e Pietikäinen (2000) & 0,6393 & 0,5271 & 0,9774 & 15,06 \\
\hline Niblack (1985) & 0,3065 & 0,8924 & 0,1901 & 5,22 \\
\hline \multicolumn{5}{|c|}{ "Banco de imagens do concurso DIBCO-2012 } \\
\hline Método vencedor do DIBCO-2012 & 0,8947 & - & - & 21,80 \\
\hline Método proposto & 0,8620 & 0,8486 & 0,8919 & 17,75 \\
\hline Su et al. (2010) & 0,8560 & 0,8402 & 0,8975 & 17,76 \\
\hline Otsu (1979) & 0,7459 & 0,8828 & 0,7297 & 14,95 \\
\hline White e Rohrer (1983) & 0,7472 & 0,6448 & 0,9513 & 16,27 \\
\hline Sauvola e Pietikäinen (2000) & 0,4938 & 0,3971 & 0,9868 & 14,67 \\
\hline Niblack (1985) & 0,2922 & 0,8851 & 0,1770 & 5,36 \\
\hline \multicolumn{5}{|c|}{ Banco de imagens do concurso DIBCO-2013 } \\
\hline Método vencedor do DIBCO-2013 & 0,9212 & - & - & 20,68 \\
\hline Método proposto & 0,8008 & 0,8064 & 0,8760 & 18,86 \\
\hline Otsu (1979) & 0,7985 & 0,7181 & 0,9668 & 19,04 \\
\hline Su et al. (2010) & 0,7681 & 0,6737 & 0,9729 & 18,52 \\
\hline White e Rohrer (1983) & 0,7312 & 0,6237 & 0,9623 & 17,56 \\
\hline Sauvola e Pietikäinen (2000) & 0,5826 & 0,4563 & 0,9983 & 16,44 \\
\hline Niblack (1985) & 0,2178 & 0,8542 & 0,1270 & 5,09 \\
\hline
\end{tabular}




\title{
Localização de textos em imagens de cenas
}

\begin{abstract}
Resumo do capítulo
Neste capítulo é proposto um método para localizar regiões de texto em imagens de cenas, o qual consiste de duas grandes etapas. Na primeira etapa, é empregado o uso dos últimos levelings para extrair regiões de caracteres da imagem de referência e, em seguida, esses caracteres são agrupados formando assim um conjunto de regiões de textos candidatas. Na segunda etapa, um conjunto de atributos é obtido a partir de cada região de texto candidata e é usado mais tarde como entrada para um classificador, a fim de rotular essas regiões em texto ou não-texto. Experimentos realizados com imagens da base pública ICDAR mostram que este método é uma boa alternativa para os problemas envolvendo a localização de textos em imagens.
\end{abstract}

\subsection{Introdução}

Nos últimos anos, diversos algoritmos para extração de informações textuais (TIE - do inglês, text information extraction) em imagens e vídeos têm sido propostos na literatura para propósitos gerais, a extração de textos em cenas urbanas e indexação baseada em conteúdo em imagens e vídeos, e para propósitos específicos como, por exemplo, extração do número do licenciamento em placa veicular e extração de endereço em cartão postal. A despeito de tais estudos, ainda não é fácil projetar um método geral de TIE para imagens de cenas, uma vez que a ocorrência de texto nessas imagens pode diferir significativamente quanto a sua inclinação, seu tamanho, seu estilo de fonte, sua iluminação e também a ocorrência de oclusões parciais. Na Figura 10.1 são apresentadas algumas imagens que fazem parte dessa categoria.
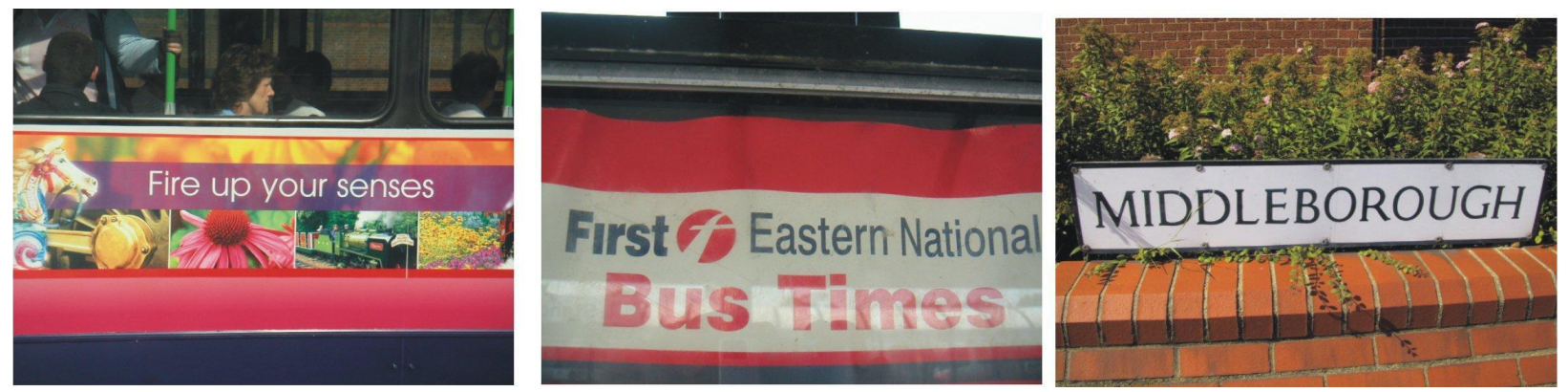

Figura 10.1: Imagens de cena do banco de imagens ICDAR (Lucas et al., 2003)

De acordo com Jung et al. (2004), o problema de TIE pode ser subdividido em cinco subproblemas, sendo eles: $(i)$ deteç̧ão; (ii) localização; (iii) rastreamento; $(i v)$ extração e realce; e $(v)$ 
reconhecimento. O método apresentado neste capítulo, baseado nos últimos levelings, está voltado para o subproblema de localização de texto, cujo desafio central é localizar regiões textuais na imagem de referência.

\subsection{Abordagem proposta}

O método proposto consiste de duas etapas principais (ver a Figura 10.2): extração e localização de regiões de texto candidatas e classificação de regiões de texto. Na primeira etapa, o objetivo é extrair um conjunto de regiões de texto candidatas a partir da imagem de referência usando um operador último leveling com filtragem que preserva regiões de caracteres e, depois, esses caracteres são agrupados formando assim um conjunto de regiões de textos candidatas. Já a segunda etapa emprega o método T-HOG, proposto por Minetto et al. (2013), para extrair um conjunto de características das regiões de texto candidatas com base no descritor HOG (do inglês, histogram of oriented gradients), para depois, classificá-las em regiões de texto ou não-texto por meio de um classificador baseado em SVM (do inglês, support vector machine).

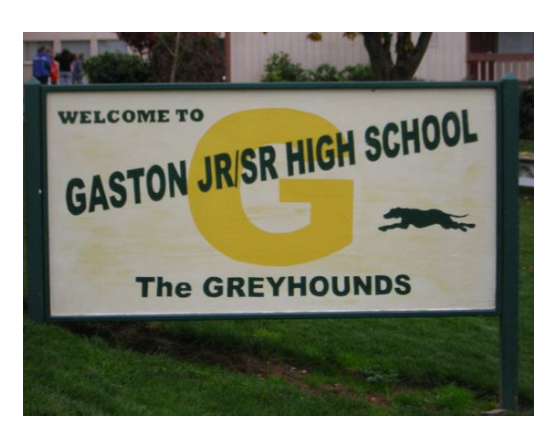

Imagem de entrada

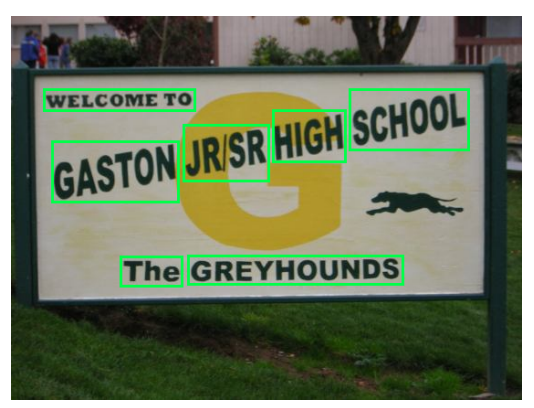

Imagem de saída

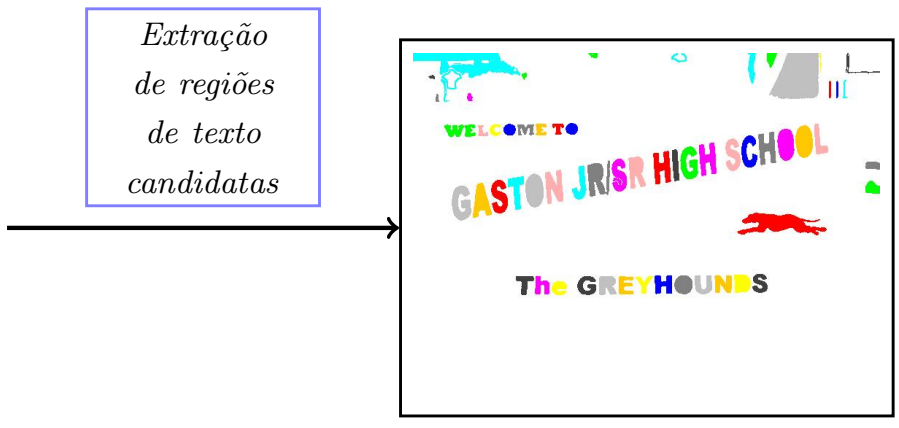

Extração de CCs

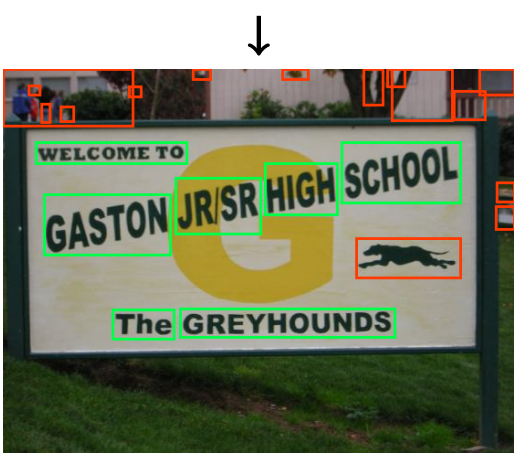

Regiões candidatas

Figura 10.2: Visão geral do método proposto.

\section{Extração de regiões candidatas}

Para extrair um conjunto de regiões de texto candidatas é construído um último leveling com base nas seguintes considerações: (i) os objetos (caracteres) das imagens de cenas podem estar nas duas polaridades da imagem, então as primitivas utilizadas são reconstruções por formas; (ii) como pretende-se segmentar os caracteres, é importante considerar todo o espaço de primitivas possíveis, e sobre o espaço dos resíduos $\left\{r_{i}\left(\varphi_{\mathcal{B}}^{T H}(f)\right): i \in \mathcal{I}\right\}$ (ou melhor, dos conjuntos $\{\mathcal{N} r(i): i \in \mathcal{I}\}$ ) é projetado um filtro que preserve vértices maximamente estáveis que podem estar presentes nos conjuntos $\mathcal{N} r(i), i \in \mathcal{I}$. Além disso, são incluídos no filtro heurísticas relacionadas à geometria dos 
caracteres a fim de filtrar regiões que não correspondem aos caracteres. Mais precisamente:

$$
\Omega_{\text {text }}(C)= \begin{cases}\text { não filtrar, } & \text { se } C \text { é MSER e } H_{1}(C), H_{2}(C), \ldots, H_{7}(C) \text { são verdadeiros, } \\ \text { filtrar, } & \text { caso contrário, }\end{cases}
$$

onde $H_{1}, H_{2}, \ldots, H_{7}$ são tradicionais heurísticas empregadas em problemas de localização de texto (Alves e Hashimoto, 2010; Neumann e Matas, 2011; Retornaz e Marcotegui, 2007; Wu et al., 2008), isto é,

$$
\begin{aligned}
& H_{1}(C)=100 \leq \operatorname{Arrea}(C) \leq \frac{|\mathcal{D}|}{3} \\
& H_{2}(C)=20 \leq \operatorname{Altura}(C) \leq \frac{\operatorname{Altura}(\mathcal{D})}{3} \\
& H_{3}(C)=10 \leq \operatorname{Largura}(C) \leq \frac{\operatorname{Largura}(\mathcal{D})}{3} \\
& H_{4}(C)=0 \leq \operatorname{Holes}(C) \leq 10 \\
& H_{5}(C)=0 \leq \frac{\max \{\operatorname{Largura}(C), \operatorname{Altura}(C)\}}{\min \{\operatorname{Largura}(C), \operatorname{Altura}(C)\}} \leq 8 \\
& H_{6}(C)=0,3 \leq \frac{\operatorname{Arrea}(C)}{\operatorname{Largura}(C) \times \operatorname{Altura}(C)} \leq 0,95 \\
& H_{7}(C)=0 \leq \operatorname{Variância}(C) \leq 60
\end{aligned}
$$

tal que a Área $(C)$, a Largura $(C)$ e a $\operatorname{Altura}(C)$ são respectivamente, a área, a largura e a altura do retângulo envolvente sobre o $\mathrm{CC} C$, e Holes $(C)$ e Variância $(C)$ denotam os números de buracos e a variância dos níveis de cinza sobre o CC $C$.

Dessa forma, considere $\mathcal{R}_{\Omega}$ o último leveling com filtragem $\Omega_{\text {text }}$ e seja $q$ a função associada ao último leveling $\mathcal{R}_{\Omega}$, que contém os índices das primitivas que produziram os máximos resíduos não nulos. Assim, os caracteres segmentados são dados da seguinte forma: $\forall p \in \mathcal{D}$, se $q(p)>0$ então $p$ é um pixel que pertence ao objeto, caso contrário, $p$ pertence ao plano de fundo da imagem. Em seguida, os vértices são agrupados em regiões de texto da seguinte forma: considere $\left\{R_{1}, R_{2}, \ldots, R_{n}\right\}$ o conjunto dos retângulos envolventes sobre os vértices (com resíduos não nulos) que deu origem a $\mathcal{R}_{\Omega}$. Então, dois retângulos $R_{i}$ e $R_{j}$ pertencem a mesma região de texto, se seus posicionamentos, alturas e alinhamentos são similares (Alves e Hashimoto, 2010; Wu et al., 2008), ou seja:

1. Os centroides de $R_{i}$ e $R_{j}$ são próximos, isto é,

$$
d_{\text {centróide }}\left(R_{i}, R_{j}\right)=\operatorname{dist}\left(\operatorname{Centróide}\left(R_{i}\right), \operatorname{Centróide}\left(R_{j}\right)\right)<\max \left\{\operatorname{Largura}\left(R_{i}\right), \operatorname{Largura}\left(R_{j}\right)\right\},
$$

onde dist é a distância euclidiana das coordenadas dos centroides de $R_{i}$ e $R_{j}$, respectivamente.

2. As alturas de $R_{i}$ e $R_{j}$ são semelhantes, ou seja,

$$
d_{\text {altura }}\left(R_{i}, R_{j}\right)=\left|\operatorname{Altura}\left(R_{i}\right)-\operatorname{Altura}\left(R_{j}\right)\right|<\min \left\{\operatorname{Altura}\left(R_{i}\right), \operatorname{Altura}\left(R_{j}\right)\right\},
$$

onde $\operatorname{Altura}\left(R_{i}\right)$ e $\operatorname{Altura}\left(R_{j}\right)$ são as alturas dos retângulos envolventes sobre os CCs $C_{i}$ e $C_{j}$, respectivamente.

3. Os centroides de $R_{i}$ e $R_{j}$ estão alinhados horizontalmente, isto é,

$$
d_{\text {linearidade }}\left(R_{i}, R_{j}\right)=\left|y_{i}-y_{j}\right|<\min \left\{\operatorname{Altura}\left(R_{i}\right), \operatorname{Altura}\left(R_{j}\right)\right\},
$$

onde $\left(x_{i}, y_{i}\right)$ e $\left(x_{j}, y_{j}\right)$ são as coordenadas dos centroides de $R_{i}$ e $R_{j}$, respectivamente. 
Baseados nesses três critérios é possível decidir se $R_{i}$ e $R_{j}$ serão fundidos ou não. Na Figura 10.3 são apresentados alguns exemplos de retângulos extraídos do últimos leveling $\mathcal{R}_{\Omega}$.
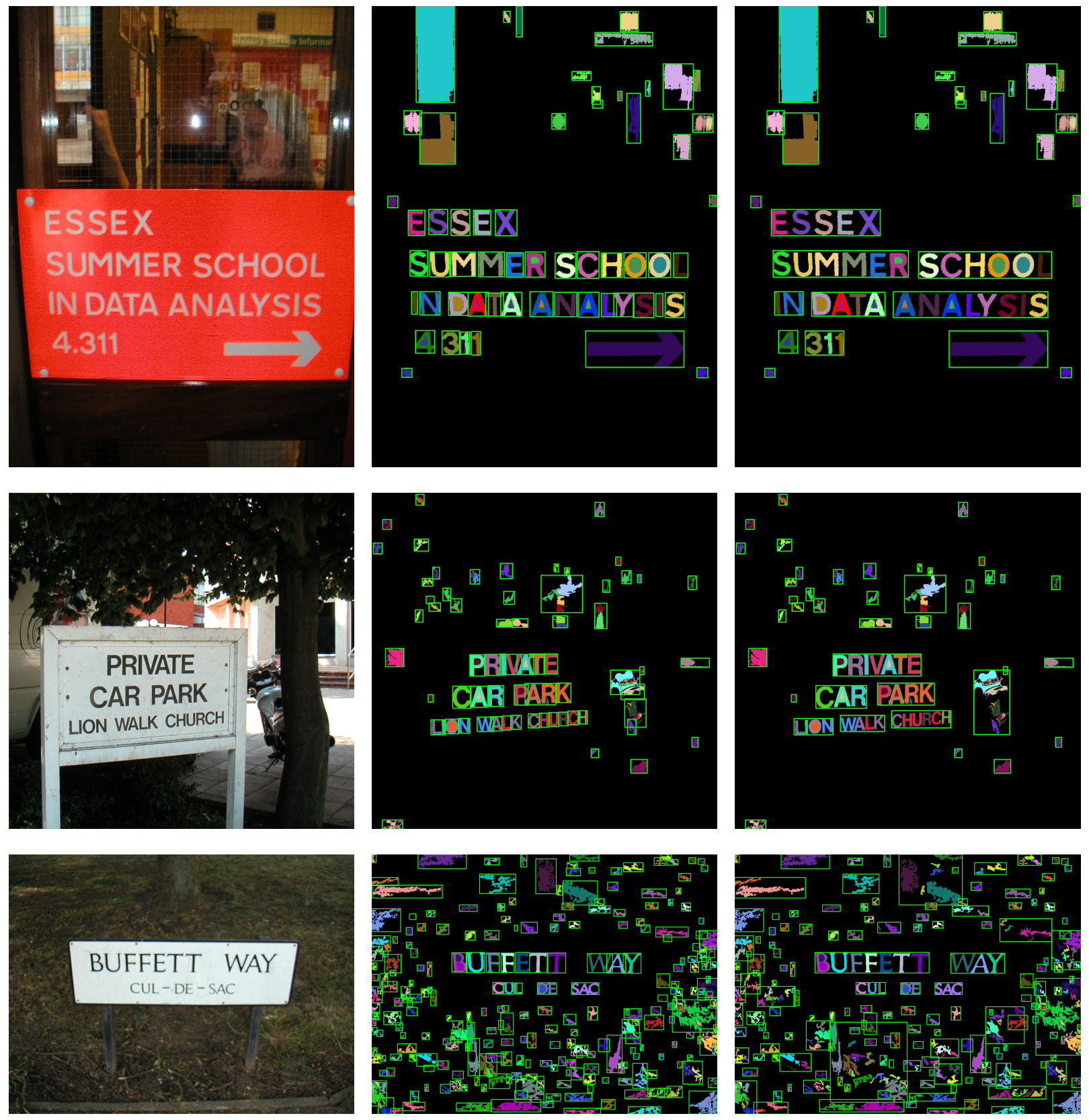

Figura 10.3: Extração das regiões de texto candidatas.

Classificação de regiões de texto

Para classificar as regiões de textos é utilizado o método T-HOG (Minetto et al., 2013), o qual consiste de um classificador de regiões de textos, originalmente proposto como um método de pós-processamento para extratores de textos, como o último levelings proposto neste capítulo. Primeiramente, o T-HOG extrai um vetor de características $z \in \mathbb{R}^{n}$ de cada região de texto candidata $R$ por meio do descritor HOG, da seguinte forma: $(i)$ o retângulo da região de texto candidata é particionado em 7 células distintas; (ii) para cada célula é calculado o HOG composto por 9 bins; (iii) o conjunto dos HOGs constitui o vetor de características $z \in \mathbb{R}^{63}$ para região de texto candidata $R$. Este vetor de características $z$ extraído pelo T-HOG é submetido para um classificador 
SVM que devolve um score (valor real), de tal forma que os scores positivos indicam a classe texto e os scores negativos indicam a classe não-texto. O classificador SVM é visto como uma função $f_{\mathrm{Svm}}: \mathbb{R}^{n} \rightarrow \mathbb{R}$ definida por

$$
f_{\mathrm{SVm}}(z)=\sum_{i=1}^{M} \alpha_{i} k\left(z_{i}, z\right)-b,
$$

onde $k$ é uma função kernel gaussiana (ou seja, $k: \mathbb{R}^{n} \times \mathbb{R}^{n} \rightarrow \mathbb{R}$ ), $M$ é a quantidade de vetores de suporte, $\alpha_{i}$ são os pesos dos vetores de suporte $z_{i}$ e $b$ é um limiar (bias). Os vetores de suporte e seus pesos são determinado pelo algoritmo de treinamento SMO (Platt, 1998), utilizando 2445 amostras, sendo que 897 é da classe texto e 1548 é não-texto, extraídas das imagens de treinamento do concurso ICDAR. A tradicional técnica de validação cruzada tem sido usada para validar a performance do classificador construído (Duda et al., 2000). Após o treinamento, a taxa de acerto do classificador foi de $98.43 \%$. Para mais detalhes sobre T-HOG e a construção do classificador podem ser encontradas em Minetto et al. (2013). Na Figura 10.4 são apresentados os resultados do classificador (classe região de texto) para os retângulos da Figura 10.3.
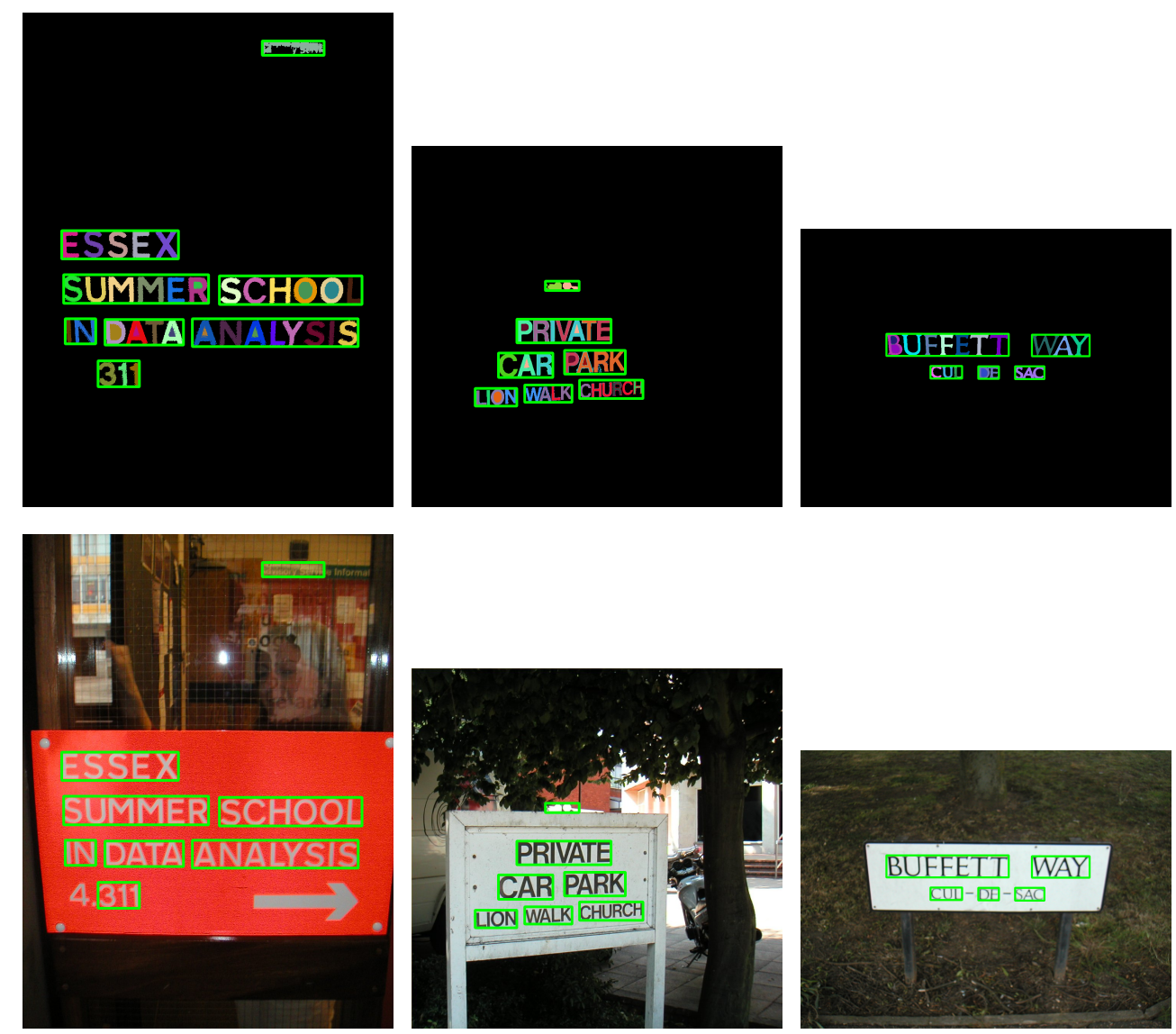

Figura 10.4: Resultados da classificação de regiões de texto usando o T-HOG.

\subsection{Experimentos e resultados}

Para avaliar o desempenho do método proposto foram utilizadas as imagens do concurso ICDAR. A base de imagens ICDAR foi originalmente criada para uma competição de localização de textos conhecida como ICDAR- 2003 (Lucas et al., 2003) e posteriormente foi utilizada em uma outra versão da mesma competição denominada ICDAR- 2005 (Lucas, 2005). Apesar de vários trabalhos de localização de texto terem sido publicados após o término da competição, muitos deles ainda 
utilizam o conjunto de imagens de ICDAR para divulgar a eficiência dos seus métodos. A base de imagens ICDAR é composta por dois conjuntos de imagens denominadas TrialTrain e TrialTest. O conjunto TrialTrain, composto por 258 imagens, é usado para ajustar ou treinar o método a ser avaliado com o conjunto TrialTest, o qual contém 251 imagens. Vale salientar, que o classificador THOG foi treinado com as regiões de texto das imagens do conjunto TrialTrain. As imagens de cenas contidas em ICDAR variam entre $307 \times 93$ a $1280 \times 960$ pixels, e as regiões de texto presentes em tais imagens apresentam diferentes características em relação a escala, orientação, cores, iluminação e oclusão. Na Figura 10.5 são apresentadas algumas imagens dos conjuntos TrialTrain e TrialTest.

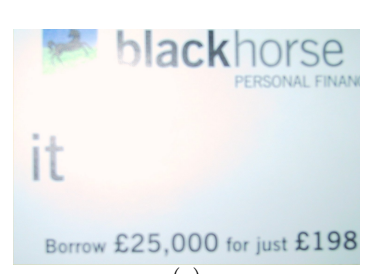

(a)

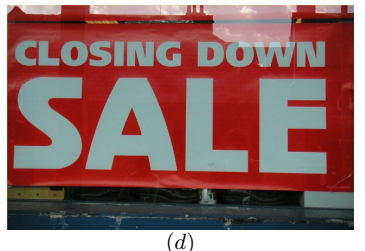

$(d)$

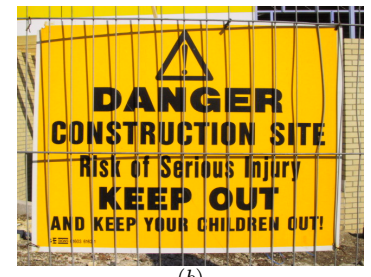

$(b)$

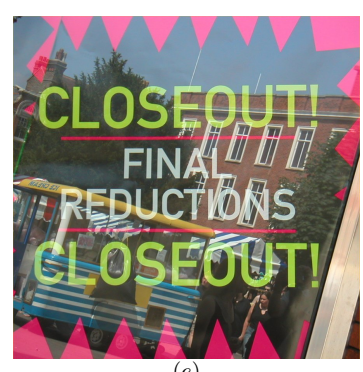

(e)

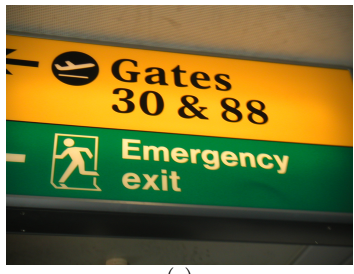

$(c)$

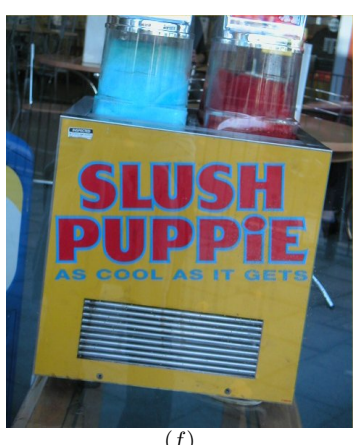

$(f)$

Figura 10.5: Exemplos de imagens da base ICDAR. $(a-c)$ imagens do conjunto TrialTrain; $(d-f)$ imagens do conjunto TrialTest.

Para avaliar o método proposto, é utilizada a mesma metodologia empregada na competição ICDAR-2003 e ICDAR-2005. Nessas competições, cada método reproduz como saída um conjunto de retângulos $E$, onde cada retângulo $r_{e} \in E$ delimita uma cadeia de caracteres localizada pelo método. Esse conjunto $E$ de retângulos de saídas é denominado estimate. Um outro conjunto $T$ de retângulos verdadeiros, denominados targets foi fornecido pelo ICDAR. A partir desses dois conjuntos de retângulos calcula-se a correspondência $m_{p}$ entre dois retângulos $r_{e} \in E$ e $r_{t} \in T$ como sendo

$$
m_{p}\left(r_{e}, r_{t}\right)=\frac{2 \times\left|r_{e} \cap r_{t}\right|}{\left|r_{e}\right|+\left|r_{t}\right|}
$$

onde $|r|$ é a área do retângulo $r$. O resultado da correspondência $m_{p}\left(r_{e}, r_{t}\right)$ é igual a 1 , quando os dois retângulos são idênticos e, 0 quando os retângulos não têm intersecção. Para cada retângulo $r_{e} \in E$, é encontrada uma correspondência mais próxima no conjunto $T$, e vice-versa. Portanto, a melhor correspondência $m(r, R)$ para um retângulo $r$ em um conjunto de retângulos $R$ é definido como sendo

$$
m(r, R)=\max _{r_{i} \in R}\left\{m_{p}\left(r, r_{i}\right)\right\} .
$$

Então, a taxa de acerto $r$ e taxa de precisão $p$ são definidas como sendo:

$$
r=\frac{\sum_{r_{t} \in T} m\left(r_{t}, E\right)}{|T|}
$$




$$
p=\frac{\sum_{r_{e} \in E} m\left(r_{e}, T\right)}{|E|}
$$

É também adotada uma média harmônica $f_{m}$ para combinar os valores de taxa de acerto e taxa de precisão em uma única medida de qualidade. O peso relativo dessa medida é controlado pelo parâmetro $\alpha$, cujo valor é igual a 0,5 , o que significa que a taxa de acerto e a taxa de precisão tem pesos iguais. A média harmônica $f_{m}$ é definida como sendo

$$
f_{m}=\frac{1}{\frac{\alpha}{p}+\frac{1-\alpha}{r}}
$$

Na Tabela 10.1 apresenta-se uma comparação dos métodos encontrados na literatura, incluindo o método proposto, que foram avaliados com as imagens de ICDAR e sua métrica.

\begin{tabular}{|c|c|c|c|}
\hline Método & Precisão & Acerto & Média harmônica \\
\hline $\operatorname{Pan}^{7}$ & $67 \%$ & $71 \%$ & $69 \%$ \\
\hline SnooperText $^{9}$ & $74 \%$ & $63 \%$ & $68 \%$ \\
\hline Epshtein $^{5}$ & $73 \%$ & $60 \%$ & $66 \%$ \\
\hline Hinnerk Becker ${ }^{4}$ & $62 \%$ & $67 \%$ & $64 \%$ \\
\hline Alex Chen ${ }^{4}$ & $60 \%$ & $60 \%$ & $60 \%$ \\
\hline Método proposto & $54 \%$ & $60 \%$ & $57 \%$ \\
\hline Alves ${ }^{8}$ & $55 \%$ & $51 \%$ & $53 \%$ \\
\hline Ashida ${ }^{3}$ & $55 \%$ & $46 \%$ & $50 \%$ \\
\hline Retornaz $^{6}$ & $41 \%$ & $57 \%$ & $48 \%$ \\
\hline HWDavid $^{3}$ & $44 \%$ & $46 \%$ & $45 \%$ \\
\hline Wolf $^{3}$ & $30 \%$ & $44 \%$ & $36 \%$ \\
\hline Qiang Zhu ${ }^{4}$ & $33 \%$ & $40 \%$ & $36 \%$ \\
\hline Jisoo Kim $^{4}$ & $22 \%$ & $28 \%$ & $25 \%$ \\
\hline Nobuo Ezaki ${ }^{4}$ & $18 \%$ & $36 \%$ & $24 \%$ \\
\hline Todoran $^{3}$ & $19 \%$ & $18 \%$ & $18 \%$ \\
\hline Full $^{3}$ & $10 \%$ & $6 \%$ & $8 \%$ \\
\hline
\end{tabular}

Tabela 10.1: Tabela comparativa de diferentes métodos para localização de textos.

\subsection{Conclusão}

Neste capítulo foi apresentada uma aplicação dos últimos levelings em um problema de localização de texto em imagens de cenas. Resultados experimentais utilizando as imagens e métricas do concurso ICDAR evidenciam que a abordagem proposta tem resultados comparáveis a outras abordagens para localização de texto. O tempo médio para o processamento de uma imagem ficou em torno de 4.3 segundos em um computador com processador $i 7$ e $8 G B$ de memória RAM.

\footnotetext{
${ }^{3}$ Resultado reportado em Lucas et al. (2003)

${ }^{4}$ Resultado reportado em Lucas (2005)

${ }^{5}$ Resultado reportado em Epshtein et al. (2010)

${ }^{6}$ Resultado reportado em Retornaz e Marcotegui (2007)

${ }^{7}$ Resultado reportado em Pan et al. (2009)

${ }^{8}$ Resultado reportado em Alves e Hashimoto (2010)

${ }^{9}$ Resultado reportado em Minetto et al. (2014)
} 
Nesta tese foi apresentada a classe dos operadores residuais, últimos levelings, os quais consistem de poderosos operadores residuais definidos a partir de resíduos gerados por operadores levelings consecutivos de um espaço de escala baseado em levelings. Assim, objetos contrastantes podem ser detectados se relevantes resíduos são gerados quando eles são filtrados por um desses levelings. Com base nessas considerações, as principais contribuições originais desta tese, foram: $(i)$ mostrar que árvores construídas a partir de conjuntos de níveis representam espaços de escalas baseados em levelings; (ii) introduzir a classe dos últimos levelings, passando por definições, conceitos, algoritmos, propriedades e relações com outros operadores conhecidos na literatura; (iii) apresentar algumas estratégias para construção de operadores últimos levelings; (iv) desenvolver aplicações baseadas em últimos levelings para problemas de análise e processamento de imagens. Além disso, foram desenvolvidos plugins para o popular software livre ImageJ que dão acesso a alguns operadores últimos levelings apresentados nesta tese.

\subsection{Trabalhos futuros}

A partir deste trabalho, novas pesquisas podem ser desenvolvidas. Neste sentido é apontada a seguir uma lista de sugestões para continuação deste trabalho:

- Estender os últimos levelings para imagens coloridas e, em especial, verificar a possibilidade de mudança do espaço das cores, como o proposto por Chong et al. (2008).

- Estender o conceito dos últimos levelings. Os últimos levelings extraem os máximos resíduos presentes na imagem para cada pixels. Este resíduo é calculado com base nas diferenças entre níveis de cinza. Neste sentido, podemos utilizar outras informações, além do nível de cinza do pixel, para calcular o valor do resíduo, como por exemplo, uma informação de similaridade da CC contendo o pixel.

- Relacionar teorias baseadas em decomposição de conjuntos de níveis para extração de objetos (como por exemplo: attribute profiles (Mura et al., 2010), filtros baseados em vetor de atributos (Urbach et al., 2005) e espaço de formas baseado em árvores (Xu, 2013)) com os últimos levelings. 
Parte IV

Apêndices 


\section{Produções durante o doutorado}

\section{Publicações}

- Alves, W. A. L., Morimitsu, A. e Hashimoto, R. F., Extraction of numerical residues in families of levelings by tree of shapes. IX Workshop de visão computacional, Rio de Janeiro-RJ, 2013.

- Alves, W. A. L., Morimitsu, A., Castro, J. S. e Hashimoto, R. F., Extraction of numerical residues in families of levelings. XXVI Conference on Graphics, Patterns and Images, (SIBGRAPI), Arequipa-Peru, 2013.

- Alves, W.A.L., Hashimoto, R., Ultimate grain filter. IEEE International Conference on Image Processing (ICIP), Paris, France, pp. 2953-2957, 2014.

- Alves, W.A.L., Morimitsu, A., Hashimoto, R.F., Scale-space representation based on levelings through hierarchies of level sets. ISMM 2015. Lecture Notes in Computer Science, vol. 9082, pp. 265-276. Springer, Heidelberg (2015).

- Morimitsu, A., Alves, W.A.L., Hashimoto, R.F.: Incremental and Efficient Computation of Families of Component Trees. ISMM 2015. Lecture Notes in Computer Science, vol. 9082, pp. 681-692. Springer, Heidelberg (2015). 
APÊNDICE A 


\section{Referências Bibliográficas}

Al-Diri et al. (2009) B. Al-Diri, A. Hunter e D. Steel. An active contour model for segmenting and measuring retinal vessels. Medical Imaging, IEEE Transactions on, 28(9):1488-1497. ISSN 0278-0062. doi: 10.1109/TMI.2009.2017941. Citado na pág. 102, 106

Alves e Hashimoto (2014) Wonder A. L. Alves e Ronaldo Hashimoto. Ultimate grain filter. Em 2014 IEEE International Conference on Image Processing (ICIP) (ICIP 2014), páginas 29532957, Paris, France. Citado na pág. 2, 3, 4, 34, 40, 51, 65, 70, 71

Alves et al. (2015) Wonder A. L. Alves, Alexandre Morimitsu e Ronaldo F. Hashimoto. Scalespace representation based on levelings through hierarchies of level sets. Em Proceedings of the 12th International Symposium on Mathematical Morphology and its Applications to Image and Signal Processing, ISMM '15. Citado na pág. 53

Alves e Hashimoto (2010) Wonder Alexandre Luz Alves e Ronaldo Fumio Hashimoto. Text regions extracted from scene images by ultimate attribute opening and decision tree classification. Em Proceedings of the 23rd SIBGRAPI Conference on Graphics, Patterns and Images, páginas 360-367. Citado na pág. 5, 51, 115, 119

Alves et al. (2013) Wonder Alexandre Luz Alves, Alexandre Morimitsu, Joel Sánchez Castro e Ronaldo Fumio Hashimoto. Extraction of numerical residues in families of levelings. Em Kim Boyer, Nina Hirata, Luciana Nedel e Claudio Silva, editors, Proceedings..., Los Alamitos. Conference on Graphics, Patterns and Images, 26. (SIBGRAPI), IEEE Computer Society's Conference Publishing Services. URL http://urlib.net/sid.inpe.br/sibgrapi/2013/07.14.21.20. Citado na pág. 3, $4,34,40,65,70$

Banon e Barrera (1994) G. J. F. Banon e J. Barrera. Bases da morfologia matemática para analise de imagens binárias. Escola de Computação, Recife. Citado na pág. 1, 12, 13

Berger et al. (2007) Ch Berger, T. Géraud, R. Levillain, N. Widynski, A. Baillard e E. Bertin. Effective component tree computation with application to pattern recognition in astronomical imaging. Em Image Processing, 200\%. ICIP 200\%. IEEE International Conference on, volume 4, páginas IV - 41-IV - 44. doi: 10.1109/ICIP.2007.4379949. Citado na pág. 37, 38, 39, 40, 43, 47

Beucher (1994) Serge Beucher. Digital skeletons in euclidean and geodesic spaces. Signal Processing, 38(1):127 - 141. ISSN 0165-1684. doi: http://dx.doi.org/10.1016/0165-1684(94) 90061-2. URL http://www.sciencedirect.com/science/article/pii/0165168494900612. Mathematical Morphology and its Applications to Signal Processing. Citado na pág. 2, 3

Beucher (2007) Serge Beucher. Numerical residues. Image Vision Comput., 25(4):405-415. ISSN 0262-8856. doi: http://dx.doi.org/10.1016/j.imavis.2006.07.020. Citado na pág. 2, 3, 65, 69, 70, 71, 80

Breen e Jones (1996) Edmond J. Breen e Ronald Jones. Attribute openings, thinnings, and granulometries. Computer Vision and Image Understanding, 64(3):377 - 389. ISSN 1077-3142. doi: http://dx.doi.org/10.1006/cviu.1996.0066. URL http://www.sciencedirect.com/science/article/ pii/S1077314296900661. Citado na pág. 16 
Cardelino et al. (2006) J. Cardelino, G. Randall, M. Bertalmio e V. Caselles. Region based segmentation using the tree of shapes. Em Image Processing, 2006 IEEE International Conference on, páginas 2421-2424. doi: 10.1109/ICIP.2006.312951. Citado na pág. 40

Carlinet e Géraud (2014) E. Carlinet e T. Géraud. A comparative review of component tree computation algorithms. Image Processing, IEEE Transactions on, 23(9):3885-3895. ISSN 10577149. doi: 10.1109/TIP.2014.2336551. Citado na pág. 37, 38, 40

Caselles e Monasse (2002) Vicent Caselles e Pascal Monasse. Grain filters. Journal of Mathematical Imaging and Vision, 17(3):249-270. Citado na pág. 16, 40, 63, 64

Caselles e Monasse (2010) Vicent Caselles e Pascal Monasse. Geometric description of images as topographic maps. Lecture Notes in Mathematics. Citado na pág. 41, 42, 56

Caselles et al. (2008) Vicent Caselles, Enric Meinhardt e Pascal Monasse. Constructing the tree of shapes of an image by fusion of the trees of connected components of upper and lower level sets. Positivity, 12(1):55-73. ISSN 1385-1292. doi: 10.1007/s11117-007-2150-2. Citado na pág. 42, 57

Chong et al. (2008) Hamilton Y. Chong, Steven J. Gortler e Todd Zickler. A perception-based color space for illumination-invariant image processing. ACM Trans. Graph., 27(3):61:1-61:7. ISSN 0730-0301. doi: 10.1145/1360612.1360660. URL http://doi.acm.org/10.1145/1360612.1360660. Citado na pág. 121

Couprie e Bertrand (1997) Michel Couprie e Gilles Bertrand. Topological grayscale watershed transformation. Em IN SPIE VISION GEOMETRY V PROCEEDINGS, 3168, páginas 136-146. Citado na pág. 34

Couprie et al. (2005) Michel Couprie, Laurent Najman e Gilles Bertrand. Quasi-linear algorithms for the topological watershed. Journal of Mathematical Imaging and Vision, 22(2-3): 231-249. ISSN 0924-9907. doi: 10.1007/s10851-005-4892-4. URL http://dx.doi.org/10.1007/ s10851-005-4892-4. Citado na pág. 34

Crozet e Géraud (2014) S. Crozet e T. Géraud. A first parallel algorithm to compute the morphological tree of shapes of nd images. Em Image Processing (ICIP), 2014 IEEE International Conference on, páginas 2933-2937. doi: 10.1109/ICIP.2014.7025593. Citado na pág. 47

Desolneux et al. (2001) Agnès Desolneux, Lionel Moisan e Jean-Michel Morel. Edge detection by helmholtz principle. Journal of Mathematical Imaging and Vision, 14(3):271-284. ISSN 09249907. doi: 10.1023/A:1011290230196. URL http://dx.doi.org/10.1023/ACitadonapág. 40

Donoser e Bischof (2006) M. Donoser e H. Bischof. Efficient maximally stable extremal region (mser) tracking. Em Computer Vision and Pattern Recognition, 2006 IEEE Computer Society Conference on, volume 1, páginas 553-560. doi: 10.1109/CVPR.2006.107. Citado na pág. 94

Dougherty e Lotufo (2003) Edward R. Dougherty e Roberto A. Lotufo. Hands-on Morphological Image Processing. SPIE Press, Bellingham, WA. ISBN 0-8194-4720-X. Citado na pág. 1, 2, 12, 13, 16,61

Duda et al. (2000) Richard O. Duda, Peter E. Hart e David G. Stork. Pattern Classification. Wiley-Interscience, second edition ed. Citado na pág. 117

Epshtein et al. (2010) Boris Epshtein, Eyal Ofek e Yonatan Wexler. Detecting text in natural scenes with stroke width transform. The Twenty-Third IEEE Computer Society Conference on Computer Vision and Pattern Recognition. Citado na pág. 119 
Fabrizio e Marcotegui (2009) Jonathan Fabrizio e Beatriz Marcotegui. Fast implementation of the ultimate opening. Em Proceedings of the 9th International Symposium on Mathematical Morphology, ISMM '09, páginas 272-281. ISBN 978-3-642-03612-5. doi: http://dx.doi.org/10. 1007/978-3-642-03613-2_25. Citado na pág. 3, 34, 70, 78

Falcao et al. (2001) A. X. Falcao, Bruno S. Cunha e Roberto A. Lotufo. Design of connected operators using the image foresting transform, 2001. URL http://dx.doi.org/10.1117/12.431120. Citado na pág. 61,62

Falcão et al. (2000) A.X. Falcão, J.K. Udupa e F.K. Miyazawa. An ultra-fast user-steered image segmentation paradigm: live wire on the fly. Medical Imaging, IEEE Transactions on, 19(1): 55-62. ISSN 0278-0062. doi: 10.1109/42.832960. Citado na pág. 40

Garrido (2002) L. Garrido. Hierarchical Region Based Processing of Images and Video Sequences: Application to Filtering, Segmentation and Information Retrieval. phd, Universitat Politècnica de Catalunya (UPC). URL http://hdl.handle.net/10803/6878. Citado na pág. 55

Gatos et al. (2009) B. Gatos, K. Ntirogiannis e I. Pratikakis. Icdar 2009 document image binarization contest (dibco 2009). Em Document Analysis and Recognition, 2009. ICDAR '09. 10th International Conference on. doi: 10.1109/ICDAR.2009.246. Citado na pág. 108, 109, 110

Grimaud (1992) Michel Grimaud. New measure of contrast: the dynamics. volume 1769, páginas 292-305. doi: 10.1117/12.60650. URL http://dx.doi.org/10.1117/12.60650. Citado na pág. 87

Géraud et al. (2013) Thierry Géraud, Edwin Carlinet, Sébastien Crozet e Laurent Najman. A quasi-linear algorithm to compute the tree of shapes of nd images. Em CrisL.Luengo Hendriks, Gunilla Borgefors e Robin Strand, editors, Mathematical Morphology and Its Applications to Signal and Image Processing, volume 7883 of Lecture Notes in Computer Science, páginas 98110. Springer Berlin Heidelberg. ISBN 978-3-642-38293-2. doi: 10.1007/978-3-642-38294-9_9. URL http://dx.doi.org/10.1007/978-3-642-38294-9_9. Citado na pág. xvii, 41, 42, 43, 44, 45, 46, 47

Heijmans (1994) H. J. A. M. Heijmans. Morphological Image Operators. Academic Press, Boston. Citado na pág. 1, 11, 12

Hernandez e Marcotegui (2011) Jorge Hernandez e Beatriz Marcotegui. Shape ultimate attribute opening. Image and Vision Computing, 29(8):533 - 545. ISSN 0262-8856. doi: DOI:10.1016/j.imavis.2011.05.001. Citado na pág. 2, 3, 4, 5, 51, 70, 71, 90, 91

Hoover et al. (2000) A. Hoover, V. Kouznetsova e M. Goldbaum. Locating blood vessels in retinal images by piecewise threshold probing of a matched filter response. Medical Imaging, IEEE Transactions on, 19(3):203-210. ISSN 0278-0062. doi: 10.1109/42.845178. Citado na pág. 101, 102, 104, 106

Jiang e Mojon (2003) Xiaoyi Jiang e D. Mojon. Adaptive local thresholding by verification-based multithreshold probing with application to vessel detection in retinal images. Pattern Analysis and Machine Intelligence, IEEE Transactions on, 25(1):131-137. ISSN 0162-8828. Citado na pág. 102,106

Jones (1999) Ronald Jones. Connected filtering and segmentation using component trees. Computer Vision and Image Understanding, 75(3):215 - 228. ISSN 1077-3142. doi: http: //dx.doi.org/10.1006/cviu.1999.0777. URL http://www.sciencedirect.com/science/article/pii/ S1077314299907774. Citado na pág. 34, 35

Jung et al. (2004) Keechul Jung, Kwang In Kim e Anil K. Jain. Text information extraction in images and video: a survey. Pattern Recognition, 37(5):977-997. Citado na pág. 113 
Keshet (2007) Renato Keshet. Adjacency lattices and shape-tree semilattices. Image and Vision Computing, 25(4):436-446. ISSN 02628856. doi: 10.1016/j.imavis.2006.04.016. URL http:// linkinghub.elsevier.com/retrieve/pii/S0262885606001752. Citado na pág. 40

Kimmel et al. (2011) R. Kimmel, Cuiping Zhang, AM. Bronstein e M.M. Bronstein. Are mser features really interesting? Pattern Analysis and Machine Intelligence, IEEE Transactions on, 33(11):2316-2320. ISSN 0162-8828. doi: 10.1109/TPAMI.2011.133. Citado na pág. 94, 96

Leite e Guimarães (2001) Neucimar J. Leite e Sílvio J. F. Guimarães. Morphological residues and a general framework for image filtering and segmentation. EURASIP J. Appl. Signal Process., 2001(1):219-229. ISSN 1110-8657. Citado na pág. 2

Lipschutz (1971) Seymour Lipschutz. Topologia geral. MCGraw-Hill do Brasil. Citado na pág. 7, 9

Lucas et al. (2003) S. M. Lucas, A. Panaretos, L. Sosa, A. Tang, S. Wong e R. Young. Icdar 2003 robust reading competitions. ICDAR03, 2:682. Citado na pág. xxi, 62, 113, 117, 119

Lucas (2005) S.M. Lucas. Icdar 2005 text locating competition results. Em Document Analysis and Recognition, 2005. Proceedings. Eighth International Conference on, páginas 80-84 Vol. 1. doi: 10.1109/ICDAR.2005.231. Citado na pág. 117, 119

Maragos e Ziff (1990) P. Maragos e R.D. Ziff. Threshold superposition in morphological image analysis systems. Pattern Analysis and Machine Intel., IEEE Trans. on, 12:498-504. ISSN 01628828. Citado na pág. 16,60

Marcotegui et al. (2011) Beatriz Marcotegui, Jorge Hernandez e Thomas Retornaz. Ultimate opening and gradual transitions. Em Mathematical Morphology and Its Applications to Image and Signal Processing, volume 6671, páginas 166-177. ISBN 978-3-642-21568-1. doi: 10.1007/ 978-3-642-21569-8_15. Citado na pág. 2, 65, 70, 86

Matas et al. (2004) J. Matas, O. Chum, M. Urban e T. Pajdla. Robust wide-baseline stereo from maximally stable extremal regions. Image and Vision Computing, 22(10):761 - 767. ISSN 0262-8856. doi: http://dx.doi.org/10.1016/j.imavis.2004.02.006. URL http://www.sciencedirect. com/science/article/pii/S0262885604000435. British Machine Vision Computing 2002. Citado na pág. $34,91,94$

Matas e Zimmermann (2005) Jiri Matas e Karel Zimmermann. A new class of learnable detectors for categorisation. Em Heikki Kalviainen, Jussi Parkkinen e Arto Kaarna, editors, Image Analysis, volume 3540 of Lecture Notes in Computer Science, páginas 541-550. Springer Berlin Heidelberg. ISBN 978-3-540-26320-3. doi: 10.1007/11499145_55. URL http://dx.doi.org/ 10.1007/11499145_55. Citado na pág. 92

Mattes et al. (1999) Julian Mattes, Mathieu Richard e Jacques Demongeot. Tree representation for image matching and object recognition. Em Gilles Bertrand, Michel Couprie e Laurent Perroton, editors, Discrete Geometry for Computer Imagery, volume 1568 of Lecture Notes in Computer Science, páginas 298-309. Springer Berlin Heidelberg. ISBN 978-3-540-65685-2. doi: 10.1007/3-540-49126-0_23. URL http://dx.doi.org/10.1007/3-540-49126-0_23. Citado na pág. 34

Mendonça e Campilho (2006) A.M. Mendonça e A. Campilho. Segmentation of retinal blood vessels by combining the detection of centerlines and morphological reconstruction. Medical Imaging, IEEE Transactions on, 25(9):1200-1213. ISSN 0278-0062. doi: 10.1109/TMI.2006. 879955. Citado na pág. 102, 106

Meyer (1998a) Fernand Meyer. The levelings. Em Proceedings of the 4th International Symposium on Mathematical Morphology and its Applications to Image and Signal Processing, ISMM '98, páginas 199-206. ISBN 0-7923-5133-9. Citado na pág. 20, 21, 26 
Meyer (1998b) Fernand Meyer. From connected operators to levelings. Em Proceedings of the 4th International Symposium on Mathematical Morphology and its Applications to Image and Signal Processing, páginas 191-198. ISBN 0-7923-5133-9. Citado na pág. 20, 21, 23, 24, 25, 27, 61, 62, 63

Meyer (2010) Fernand Meyer. Levelings and flat zone morphology. Pattern Recognition, International Conference on, 0:1570-1573. ISSN 1051-4651. doi: http://doi.ieeecomputersociety.org/ 10.1109/ICPR.2010.388. Citado na pág. 3, 4, 5, 51, 65, 70, 71

Meyer e Maragos (2000) Fernand Meyer e Petros Maragos. Nonlinear scale-space representation with morphological levelings. J. of Visual Comm. and Image Representation, 11:200-0. Citado na pág. 28

Meyer e Maragos (1999) Fernand Meyer e Petros Maragos. Morphological scale-space representation with levelings. Em in Scale-Space?99, ser. Lecture Notes in Computer Science, páginas 187-198. Springer-Verlag. Citado na pág. 19, 65, 66

Minetto et al. (2013) Rodrigo Minetto, Nicolas Thome, Matthieu Cord, Neucimar J. Leite e Jorge Stolfi. T-hog: An effective gradient-based descriptor for single line text regions. Pattern Recognition, 46(3):1078 - 1090. ISSN 0031-3203. doi: http://dx.doi.org/10.1016/j.patcog.2012. 10.009. URL http://www.sciencedirect.com/science/article/pii/S0031320312004438. Citado na pág. $114,116,117$

Minetto et al. (2014) Rodrigo Minetto, Nicolas Thome, Matthieu Cord, Neucimar J. Leite e Jorge Stolfi. Snoopertext: A text detection system for automatic indexing of urban scenes. Computer Vision and Image Understanding, 122(0):92 - 104. ISSN 1077-3142. doi: http://dx.doi.org/10.1016/ j.cviu.2013.10.004. URL http://www.sciencedirect.com/science/article/pii/S1077314213001914. Citado na pág. 119

Monasse (2000) Pascal Monasse. Morphological representation of digital images and application to registration. Tese de doutorado, Universidade de Paris-Dauphine. Citado na pág. 40, 41, 42, 43, 47

Monasse e Guichard (2000) Pascal Monasse e Frederic Guichard. Fast computation of a contrastinvariant image representation. IEEE Transactions on Image Processing, 9(5):860-872. Citado na pág. $40,41,42,43$

Mura et al. (2010) Mauro Dalla Mura, Jon Atli Benediktsson, Björn Waske e Lorenzo Bruzzone. Morphological attribute profiles for the analysis of very high resolution images. IEEE T. Geoscience and Remote Sensing, 48(10):3747-3762. doi: 10.1109/TGRS.2010.2048116. URL http://dx.doi.org/10.1109/TGRS.2010.2048116. Citado na pág. 121

Najman e Couprie (2006) Laurent Najman e Michel Couprie. Building the component tree in quasi-linear time. Image Processing, IEEE Transactions on, 15(11):3531-3539. ISSN 1057-7149. doi: 10.1109/TIP.2006.877518. Citado na pág. 34, 37, 92

Najman e Géraud (2013) Laurent Najman e Thierry Géraud. Discrete set-valued continuity and interpolation. Em CrisL.Luengo Hendriks, Gunilla Borgefors e Robin Strand, editors, Mathematical Morphology and Its Applications to Signal and Image Processing, volume 7883 of Lecture Notes in Computer Science, páginas 37-48. Springer Berlin Heidelberg. ISBN 978-3-642-38293-2. doi: 10.1007/978-3-642-38294-9_4. URL http://dx.doi.org/10.1007/978-3-642-38294-9_4. Citado na pág. $41,43,44,45,47$

Najman e Talbot (2013) Laurent Najman e Hugues Talbot. Mathematical Morphology. John Wiley \& Sons. Citado na pág. 1, 2, 11, 12

Neumann e Matas (2012) L. Neumann e J. Matas. Real-time scene text localization and recognition. Em Computer Vision and Pattern Recognition (CVPR), 2012 IEEE Conference on, páginas 3538-3545. doi: 10.1109/CVPR.2012.6248097. Citado na pág. 92 
Neumann e Matas (2011) Lukas Neumann e Jiri Matas. A method for text localization and recognition in real-world images. Em Ron Kimmel, Reinhard Klette e Akihiro Sugimoto, editors, Computer Vision? ACCV 2010, volume 6494 of Lecture Notes in Computer Science, páginas 770783. Springer Berlin Heidelberg. ISBN 978-3-642-19317-0. doi: 10.1007/978-3-642-19318-7_60. URL http://dx.doi.org/10.1007/978-3-642-19318-7_60. Citado na pág. 115

Newman (1992) Maxwell Herman Alexander Newman. Elements of the topology of plane sets of points. Dover Publications. Citado na pág. 7, 9

Niblack (1985) Wayne Niblack. An Introduction to Digital Image Processing. Strandberg Publishing Company, Birkeroed, Denmark, Denmark. ISBN 87-872-0055-4. Citado na pág. 111, 112

Niemeijer et al. (2004) Meindert Niemeijer, Joes Staal, Bram van Ginneken, Marco Loog e Michael D. Abramoff. Comparative study of retinal vessel segmentation methods on a new publicly available database, 2004. URL http://dx.doi.org/10.1117/12.535349. Citado na pág. 102, 106

Nister e Stewenius (2006) David Nister e Henrik Stewenius. Scalable recognition with a vocabulary tree. Em Proceedings of the 2006 IEEE Computer Society Conference on Computer Vision and Pattern Recognition - Volume 2, CVPR '06, páginas 2161-2168, Washington, DC, USA. IEEE Computer Society. ISBN 0-7695-2597-0. doi: 10.1109/CVPR.2006.264. URL http://dx.doi.org/10.1109/CVPR.2006.264. Citado na pág. 94

Nistér e Stewénius (2008) David Nistér e Henrik Stewénius. Linear time maximally stable extremal regions. Em David Forsyth, Philip Torr e Andrew Zisserman, editors, Computer Vision ? ECCV 2008, volume 5303 of Lecture Notes in Computer Science, páginas 183-196. Springer Berlin Heidelberg. ISBN 978-3-540-88685-3. doi: 10.1007/978-3-540-88688-4_14. URL http: //dx.doi.org/10.1007/978-3-540-88688-4_14. Citado na pág. 36, 38

Obdrzalek e Matas (2005) Stepan Obdrzalek e Jiri Matas. Sub-linear indexing for large scale object recognition. Em BMVC, páginas 1-10. Citado na pág. 94

Otsu (1979) N. Otsu. A threshold selection method from graylevel histograms. IEEE Trans. on Systems, Man and Cybernetics, 9. Citado na pág. 111, 112

Pan et al. (2009) Yi-Feng Pan, Xinwen Hou e Cheng-Lin Liu. Text localization in natural scene images based on conditional random field. Em ICDAR '09: Proceedings of the 2009 10th International Conference on Document Analysis and Recognition, páginas 6-10, Washington, DC, USA. IEEE Computer Society. ISBN 978-0-7695-3725-2. doi: http://dx.doi.org/10.1109/ICDAR.2009. 97. Citado na pág. 119

Perd'och (2011) Michal Perd'och. Maximally Stable Extremal Regions and Local Geometry for Visual Correspondences. Tese de Doutorado, Czech Technical University in Prague. Citado na pág. 96

Platt (1998) J. Platt. Fast training of support vector machines using sequential minimal optimization. Em B. Schoelkopf, C. Burges e A. Smola, editors, Advances in Kernel Methods - Support Vector Learning. MIT Press. URL http://research.microsoft.com/ jplatt/smo.html. Citado na pág. 117

Pratikakis et al. (2013) I. Pratikakis, B. Gatos e K. Ntirogiannis. Icdar 2013 document image binarization contest (dibco 2013). Em Document Analysis and Recognition (ICDAR), 2013 12th International Conference on, páginas 1471-1476. doi: 10.1109/ICDAR.2013.219. Citado na pág. 108, 109, 110 
Pratikakis et al. (2010) Ioannis Pratikakis, Basilis Gatos e Konstantinos Ntirogiannis. H-dibco 2010 - handwritten document image binarization competition. Em Proceedings of the 2010 12th International Conference on Frontiers in Handwriting Recognition, ICFHR '10, páginas 727-732, Washington, DC, USA. IEEE Computer Society. ISBN 978-0-7695-4221-8. doi: 10.1109/ICFHR. 2010.118. URL http://dx.doi.org/10.1109/ICFHR.2010.118. Citado na pág. 108, 109, 110

Pratikakis et al. (2011) Ioannis Pratikakis, Basilis Gatos e Konstantinos Ntirogiannis. Icdar 2011 document image binarization contest (dibco 2011). Em Proceedings of the 2011 International Conference on Document Analysis and Recognition, ICDAR '11, páginas 1506-1510. IEEE Computer Society. ISBN 978-0-7695-4520-2. doi: 10.1109/ICDAR.2011.299. Citado na pág. 108, 109, 110

Pratikakis et al. (2012) Ioannis Pratikakis, Basilis Gatos e Konstantinos Ntirogiannis. Icfhr 2012 competition on handwritten document image binarization (h-dibco 2012). Em Proceedings of the 2012 International Conference on Frontiers in Handwriting Recognition, ICFHR '12, páginas 817-822, Washington, DC, USA. IEEE Computer Society. ISBN 978-0-7695-4774-9. doi: 10.1109/ ICFHR.2012.216. URL http://dx.doi.org/10.1109/ICFHR.2012.216. Citado na pág. 108, 109, 110

Retornaz (2007) Thomas Retornaz. Automatic detection of text from natural scenes: a semantic descriptor for content based image retrieval. Theses, École Nationale Supérieure des Mines de Paris. URL https://pastel.archives-ouvertes.fr/pastel-00003782. Citado na pág. 90

Retornaz e Marcotegui (2007) Thomas Retornaz e Beatriz Marcotegui. Scene text localization based on the ultimate opening. Em Proceedings of the 8th International Symposium on Mathematical Morphology and its Applications to Image and Signal Processing, volume 1 of ISMM '08, páginas 177-188. ISBN 978-85-17-00035-5. Citado na pág. 3, 4, 5, 51, 65, 70, 71, 115, 119

Salembier e Serra (1995) P. Salembier e J. Serra. Flat zones filtering, connected operators, and filters by reconstruction. IEEE Transactions on Image Processing, 4:1153-1160. Citado na pág. 3, 19,20

Salembier e Wilkinson (2009) P. Salembier e M.H.F. Wilkinson. Connected operators. Signal Processing Magazine, IEEE, 26(6):136-157. ISSN 1053-5888. doi: 10.1109/MSP.2009.934154. Citado na pág. $16,55,63$

Salembier et al. (1998) Philippe Salembier, Albert Oliveras e Luis Garrido. Anti-extensive connected operators for image and sequence processing. IEEE Transactions on Image Processing, 7 (4):555-570. ISSN 1057-7149. doi: 10.1109/83.663500. Citado na pág. 16, 34, 35, 36, 38, 63, 64

Sauvola e Pietikäinen (2000) J. Sauvola e M. Pietikäinen. Adaptive document image binarization. PATTERN RECOGNITION, 33:225-236. Citado na pág. 111, 112

Serra (1982) Jean Serra. Image Analysis and Mathematical Morphology. Academic Press, Inc., New York, USA. Citado na pág. 1, 2, 11, 12, 13, 32

Serra (1988) Jean Serra. Image Analysis and Mathematical Morphology, volume 2: Theoretical Advances. Academic Press, London. Citado na pág. 2

Serra e Vincent (1992) Jean Serra e Luc Vincent. An overview of morphological filtering. Circuits, Systems and Signal Processing, 11(1):47-108. ISSN 0278-081X. doi: 10.1007/BF01189221. Citado na pág. 2, 14

Serra e Salembier (1993) Jean C. Serra e Philippe Salembier. Connected operators and pyramids. Em Image Algebra and Morphological Image Processing IV, volume 2030, páginas 65-76. Citado na pág. $19,20,24,25$ 
Silva (2009) Alexandre Gonçalves Silva. Uso de árvore de componentes para filtragem, segmentação e deteç̧ão de padrões em imagens digitais. Tese de doutorado, Universidade Estadual de Campinas. Citado na pág. 92

Silva e de Alencar Lotufo (2011) Alexandre Gonçalves Silva e Roberto de Alencar Lotufo. Efficient computation of new extinction values from extended component tree. Pattern Recognition Letters, 32(1):79 - 90. ISSN 0167-8655. doi: http://dx.doi.org/10.1016/j.patrec.2010.07.019. URL http://www.sciencedirect.com/science/article/pii/S0167865510002436. Citado na pág. 34, 88

Sivic e Zisserman (2003) Josef Sivic e Andrew Zisserman. Video google: A text retrieval approach to object matching in videos. Em Proceedings of the Ninth IEEE International Conference on Computer Vision - Volume 2, ICCV '03, páginas 1470-, Washington, DC, USA. IEEE Computer Society. ISBN 0-7695-1950-4. URL http://dl.acm.org/citation.cfm?id=946247.946751. Citado na pág. 94

Soille (2003) Pierre Soille. Morphological Image Analysis: Principles and Applications. SpringerVerlag New York, Inc., Secaucus, NJ, USA. ISBN 3540429883. Citado na pág. 1, 2, 12, 13, 16, 61

Soille e Najman (2012) Pierre Soille e Laurent Najman. On morphological hierarchical representations for image processing and spatial data clustering. Em Ullrich Köthe, Annick Montanvert e Pierre Soille, editors, Applications of Discrete Geometry and Mathematical Morphology, volume 7346 of Lecture Notes in Computer Science, páginas 43-67. Springer Berlin Heidelberg. ISBN 978-3-642-32312-6. doi: 10.1007/978-3-642-32313-3_4. URL http://dx.doi.org/10.1007/ 978-3-642-32313-3_4. Citado na pág. 34

Staal et al. (2004) J.J. Staal, M.D. Abramoff, M. Niemeijer, M.A. Viergever e B. van Ginneken. Ridge based vessel segmentation in color images of the retina. IEEE Transactions on Medical Imaging, 23(4):501-509. Citado na pág. 101, 102, 104, 106

Su et al. (2010) Bolan Su, Shijian Lu e Chew Lim Tan. Binarization of historical document images using the local maximum and minimum. Em Proceedings of the 9th IAPR International Workshop on Document Analysis Systems, DAS '10, páginas 159-166, New York, NY, USA. ACM. ISBN 978-1-60558-773-8. doi: 10.1145/1815330.1815351. URL http://doi.acm.org/10.1145/1815330. 1815351. Citado na pág. 111, 112

Tarjan (1975) Robert Endre Tarjan. Efficiency of a good but not linear set union algorithm. $J$. ACM , 22(2):215-225. ISSN 0004-5411. doi: 10.1145/321879.321884. URL http://doi.acm.org/ 10.1145/321879.321884. Citado na pág. 37, 38, 40

Urbach e Wilkinson (2002) E. R. Urbach e M. H. F. Wilkinson. Shape-only granulometries and grey-scale shape filters. Em Mathematical Morphology and Its Applications to Signal and Image Processing, volume 2002, páginas 305-314. Citado na pág. 63, 92

Urbach et al. (2007) E.R. Urbach, J.B.T.M. Roerdink e M.H.F. Wilkinson. Connected shapesize pattern spectra for rotation and scale-invariant classification of gray-scale images. Pattern Analysis and Machine Intelligence, IEEE Transactions on, 29(2):272-285. ISSN 0162-8828. doi: 10.1109/TPAMI.2007.28. Citado na pág. 91, 92

Urbach et al. (2004) Erik R. Urbach, Jos B. T. M. Roerdink e Michael H. F. Wilkinson. Connected rotation-invariant size-shape granulometries. Em Proc. 17th Int?l Conf. Pattern Recognition, páginas 688-691. Citado na pág. 63

Urbach et al. (2005) ErikR. Urbach, NiekJ. Boersma e MichaelH.F. Wilkinson. Vectorattribute filters. Em Christian Ronse, Laurent Najman e Etienne Decencière, editors, Mathematical Morphology: 40 Years On, volume 30 of Computational Imaging and Vision, páginas 
95-104. Springer Netherlands. ISBN 978-1-4020-3442-8. doi: 10.1007/1-4020-3443-1_10. URL http://dx.doi.org/10.1007/1-4020-3443-1_10. Citado na pág. 91, 92, 121

Vachier e Meyer (1995) C. Vachier e F. Meyer. Extinction value: a new measurement of persistence. Em In IEEE Workshop on Nonlinear Signal and Image Processing, páginas 254-257. Citado na pág. 87

Veltkamp (2001) R.C. Veltkamp. Shape matching: similarity measures and algorithms. Em Shape Modeling and Applications, SMI 2001 International Conference on., páginas 188-197. doi: 10.1109/SMA.2001.923389. Citado na pág. 91

Veltkamp e Hagedoorn (2000) Remco C. Veltkamp e Michiel Hagedoorn. Shape similarity measures, properties, and constructions. Em In Advances in Visual Information Systems, 4th International Conference, páginas 467-476. Springer. Citado na pág. 91

Vincent (1994) Luc Vincent. Morphological area openings and closings for grey-scale images. Em Ying-Lie O, Alexander Toet, David Foster, HenkJ.A.M. Heijmans e Peter Meer, editors, Shape in Picture, volume 126 of NATO ASI Series, páginas 197-208. Springer Berlin Heidelberg. ISBN 978-3-642-08188-0. doi: 10.1007/978-3-662-03039-4_13. URL http://dx.doi.org/10.1007/ 978-3-662-03039-4_13. Citado na pág. 16

Vincent (1993) Luc Vincent. Morphological grayscale reconstruction in image analysis: applications and efficient algorithms. Image Processing, IEEE Trans. on, 2(2):176-201. ISSN 1057-7149. Citado na pág. 60, 61, 62

Wendt et al. (1986) P. Wendt, E.J. Coyle e Jr. Gallagher, N.C. Stack filters. Acoustics, Speech and Signal Processing, IEEE Transactions on, 34(4):898-911. ISSN 0096-3518. doi: 10.1109/ TASSP.1986.1164871. Citado na pág. 16, 60

White e Rohrer (1983) J. M. White e G. D. Rohrer. Image thresholding for optical character recognition and other applications requiring character image extraction. IBM J. Res. Dev, 27: 400-411. Citado na pág. 111, 112

Wilkinson et al. (2011) MichaelH.F. Wilkinson, Pierre Soille, Martino Pesaresi e GeorgiosK. Ouzounis. Concurrent computation of differential morphological profiles on giga-pixel images. Em Pierre Soille, Martino Pesaresi e GeorgiosK. Ouzounis, editors, Mathematical Morphology and Its Applications to Image and Signal Processing, volume 6671 of Lecture Notes in Computer Science, páginas 331-342. Springer Berlin Heidelberg. ISBN 978-3-642-21568-1. doi: 10.1007/ 978-3-642-21569-8_29. URL http://dx.doi.org/10.1007/978-3-642-21569-8_29. Citado na pág. 36, 38,60

Wu et al. (2008) Jui-Chen Wu, Jun-Wei Hsieh e Yung-Sheng Chen. Morphology-based text line extraction. Machine Vision Application, 19(3):195-207. ISSN 0932-8092. Citado na pág. 115

$\mathrm{Xu}$ (2013) Yongchao Xu. Tree-based shape spaces: Definition and applications in image processing and computer vision. Tese de Doutorado, Université Paris-Est, Marne-la-Vallée, France. Citado na pág. 55, 63, 92, 121

Xu et al. (2012) Yongchao Xu, T. Géraud e L. Najman. Context-based energy estimator: Application to object segmentation on the tree of shapes. Em Image Processing (ICIP), 2012 19th IEEE International Conference on, páginas 1577-1580. doi: 10.1109/ICIP.2012.6467175. Citado na pág. 40

$\mathrm{Xu}$ et al. (2013) Yongchao Xu, Thierry Géraud e Laurent Najman. Two applications of shapebased morphology: Blood vessels segmentation and a generalization of constrained connectivity. Em CrisL.Luengo Hendriks, Gunilla Borgefors e Robin Strand, editors, Mathematical Morphology and Its Applications to Signal and Image Processing, volume 7883 of Lecture Notes in Computer 
Science, páginas 390-401. Springer Berlin Heidelberg. ISBN 978-3-642-38293-2. doi: 10.1007/ 978-3-642-38294-9_33. URL http://dx.doi.org/10.1007/978-3-642-38294-9_33. Citado na pág. 92, 102,106

$\mathrm{Xu}$ et al. (2014) Yongchao Xu, P. Monasse, T. Geraud e L. Najman. Tree-based morse regions: A topological approach to local feature detection. Image Processing, IEEE Transactions on, 23 (12):5612-5625. ISSN 1057-7149. doi: 10.1109/TIP.2014.2364127. Citado na pág. 96 


\section{Índice Remissivo}

Árvore de componentes, 34

Árvore de componentes estendida, 35

Árvores por meio de posets, 29, 30

Última abertura, 3

Última abertura por atributo, 3, 4

Última filtro por grão, 4

Último fechamento por atributo, 4

Pixel, 9

Algoritmo para construção de árvore de componentes, 36

Anti-extensividade, 12

Auto-complementar, 12

Auto-dualidade, 12

Binarização de texto em imagens de documentos históricos, 107

Caminho, 10

Complementar, 12

Componente conexo, 10

Conjunto de níveis, 32

Crescente, 12

Descritores computáveis incrementalmente, 92

Diagrama de Hasse, 30

Dualidade, 12

Elemento estruturante, 13

Espaço de escala, 3, 4

espaço de escala, 51, 69

Espaço de escala com base em levelings, 27

Extensividade, 12

Extrema regional, 11

Filtragem com base em regiões estáveis, 94

Filtragem com base em vetor de atributos, 91

Filtro morfológico, 1

Filtros morfológicos, 14, 15

Filtros por atributos, 16

Grafo da imagem, 10

Idempotência, 12

ImageJ, 5
Imagem, 9

Imagem binária, 9

Imagem de retina, 101

Imagem em níveis de cinza, 9

Imagem multibanda, 9

Localização de textos, 113

Máxima regional, 11

Mínima regional, 11

max-tree, 35

Medida de desempenho acurácia, 104

Medida de desempenho especificidade, 104

Medida de desempenho sensibilidade, 104

Menor componente, 35

min-tree, 35

Morfologia Matemática, 1

MSER, 94

MSER +, 94

MSER-, 94

Operador, 1

operador, 51, 69

Operador leveling, 21

Operador monotone planing, 21, 22

Operador abertura, 13

Operador abertura por atributo, 16

Operador conexo, 3, 19, 20

Operador de imagens, 1

Operador de subconjuntos, 1

Operador dilatação, 13

Operador dilatação condicional, 14

Operador erosão, 13

Operador erosão condicional, 14

Operador fechamento, 13

Operador fechamento por atributo, 16

Operador leveling, 4, 21

Operador limiarização, 16

Operador reconstrução por dilatação, 14

Operador reconstrução por erosão, 14

Operador residual, 2, 3, 5

Operador residual estendido, 2

Operadores de imagens, 11

Operadores de subconjuntos, 11 
Operadores morfológicos, 11

Operadores visto como relações binárias, 22

Partição, 11

Partição das zonas planas, 11

Perfil de intesidade, 11

plugins, 5

poset, 30

Primitivas, 2

Relação de adjacência, 9

Resíduo negativo, 2, 4

Resíduo nulo, 2

Resíduo positivo, 2, 4

Reticulado completo, 1

Segmentação de vasos sanguíneos, 101

Seleção de primitivas baseadas em atributos, 85

Seleção de primitivas baseadas em imagens marcadoras, 88

Seleção de primitivas baseadas em transições graduais, 86

Seleção de primitivas baseadas em valores de extinção, 87

Zona plana, 10 